Supporting Information

\title{
Asymmetric Synthesis of Axially Chiral Anilides via Organocatalytic Atroposelective $N$-Acylation
}

Dawei Li, Sijing Wang, Shulin Ge, Shunxi Dong,* Xiaoming Feng

Key Laboratory of Green Chemistry \& Technology, Ministry of Education, College of Chemistry, Sichuan University, Chengdu 610064, China

E-mail: dongs@scu.edu.cn

\section{Contents}

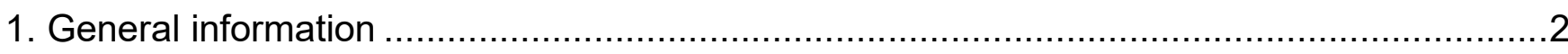

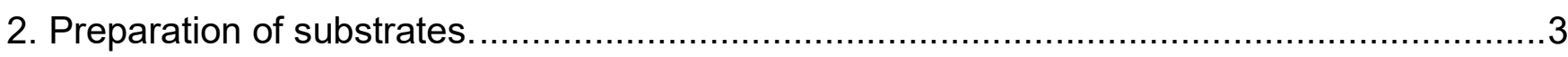

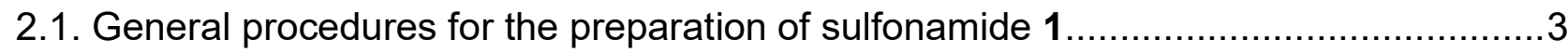

2.2. General procedures for the preparation of $\alpha, \beta$-unsaturated carbonic anhydride $2 \ldots . .7$

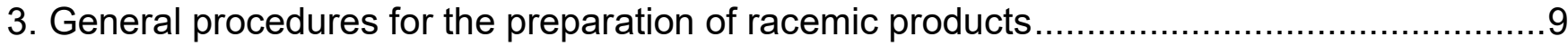

4. General procedures for the catalytic asymmetric reaction .........................................

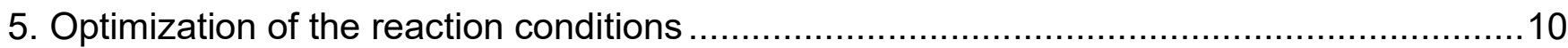

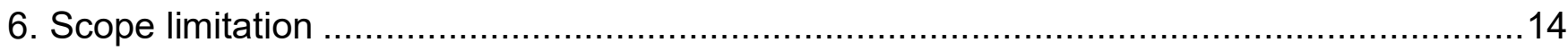

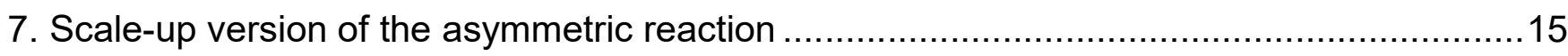

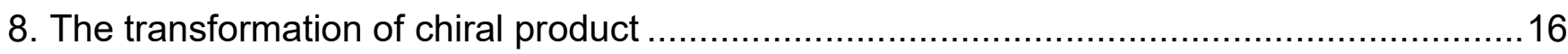

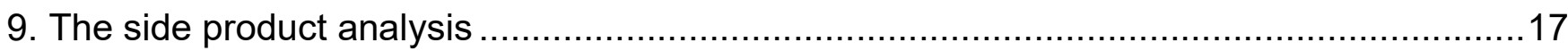

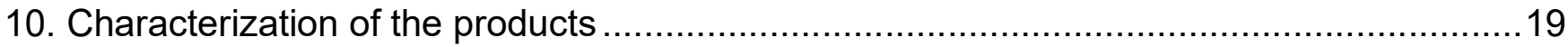

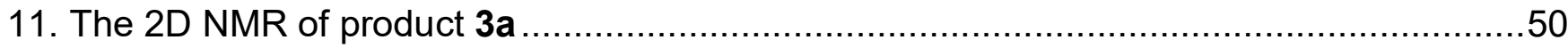

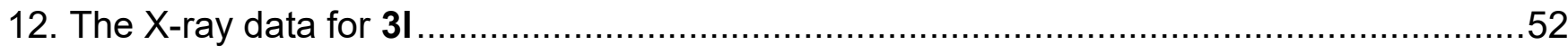

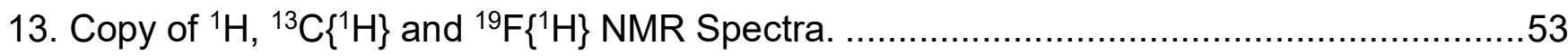

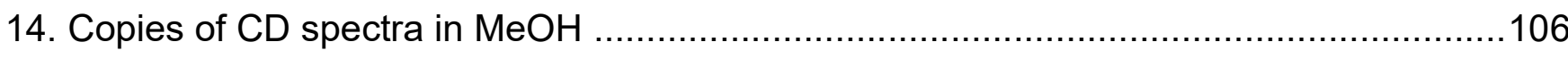

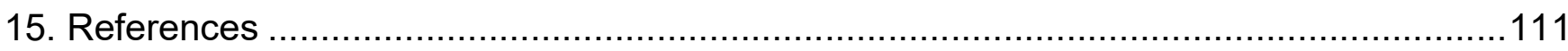




\section{General information}

${ }^{1} \mathrm{H}$ NMR spectra were recorded on bruker ASCEND ${ }^{\mathrm{TM}} 400 \mathrm{M}(400 \mathrm{MHz})$. Chemical shifts were reported in ppm from tetramethylsilane with the solvent resonance as the internal standard $\left(\mathrm{CDCl}_{3}, \delta=7.26\right)$. Spectra were reported as follows: chemical shift $(\delta$ ppm), multiplicity ( $\mathrm{s}=$ singlet, $\mathrm{d}=$ doublet, $\mathrm{t}=$ triplet, $\mathrm{q}=$ quartet, $\mathrm{m}=$ multiplet, $\mathrm{dd}=$ doublet of doublets), coupling constants $(\mathrm{Hz})$, integration and assignment. ${ }^{13} \mathrm{C}\left\{{ }^{1} \mathrm{H}\right\}$ NMR spectra were collected on bruker ASCEND ${ }^{\mathrm{TM}} 400 \mathrm{M}(101 \mathrm{MHz})$ with complete proton decoupling. Chemical shifts are reported in ppm from the tetramethylsilane with the solvent resonance as internal standard $\left(\mathrm{CDCl}_{3}, \delta=77.0\right)$. ${ }^{19} \mathrm{~F}\left\{{ }^{1} \mathrm{H}\right\}$ NMR spectra were collected on bruker ASCEND ${ }^{\mathrm{TM}} 400 \mathrm{M}(376 \mathrm{MHz})$ with complete proton decoupling. HRMS was recorded on a Thermo Q-Exactive Focus (FTMS+c ESI). Enantiomeric excesses (ee) were determined by HPLC and UPC ${ }^{2}$ analysis using the corresponding commercial chiral column as stated in the experimental procedures at $23^{\circ} \mathrm{C}$ with UV detector at $254 \mathrm{~nm}$. Optical rotations were reported as follows: $[\alpha]_{\lambda}^{\top}=\left(c=\mathrm{g} / 100 \mathrm{~mL}\right.$, in $\mathrm{CH}_{2} \mathrm{Cl}_{2}$, unless otherwise noted, $\left.\lambda=589 \mathrm{~nm}\right)$. IR was detected by Bruker Tensor II spectrometer with Plantium ATR accessory. Solvents were dried and distilled prior to use according to the standard methods. Unless otherwise indicated, the reaction was performed under nitrogen atmosphere and reagents obtained from commercial sources were used without further purification. The isothiourea catalysts were prepared according to literature procedure. ${ }^{1}$ 


\section{Preparation of substrates.}

\subsection{General procedures for the preparation of sulfonamide 1.}

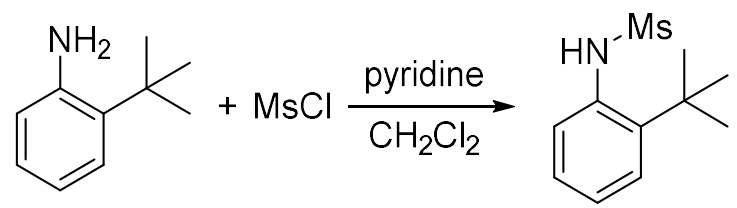

Under $\mathrm{N}_{2}$ atmosphere, methanesulfonyl chloride ( $3.3 \mathrm{mmol}$ ) was added to the solution of aniline $(3.0 \mathrm{mmol})$ and pyridine (4.5 mmol) in $\mathrm{CH}_{2} \mathrm{Cl}_{2}(5.0 \mathrm{~mL})$ at $0{ }^{\circ} \mathrm{C}$ in an ice/water bath, and the reaction mixture was stirred for $18 \mathrm{~h}$ from $0{ }^{\circ} \mathrm{C}$ to rt. The mixture was poured into $\mathrm{H}_{2} \mathrm{O}$ and extracted with EtOAc. The EtOAc extracts were washed with brine, dried over $\mathrm{MgSO}_{4}$, and evaporated to dryness. The residue was subjected to column chromatography on silica gel and eluted with hexane and EtOAc $(\mathrm{v} / \mathrm{v}=5 / 1)$ gave $1 \mathrm{~b}$ as white solid.

N-(2-(Tert-butyl)phenyl)-4-methylbenzenesulfonamide (1a)<smiles>Cc1ccc(S(=O)(=O)Nc2ccccc2C(C)(C)C)cc1</smiles>

White solid. $70 \%$ yield, 5.0 g. ${ }^{1} \mathrm{H}$ NMR $\left(400 \mathrm{MHz}, \mathrm{CDCl}_{3}\right) \delta=7.73(\mathrm{~d}, J=8.4,2 \mathrm{H}), 7.43(\mathrm{dd}, \mathrm{J}=8.0,1.5,1 \mathrm{H}), 7.31(\mathrm{dd}, J=7.9,1.7$, $1 \mathrm{H}), 7.28-7.22(\mathrm{~m}, 2 \mathrm{H}), 7.15-7.02(\mathrm{~m}, 2 \mathrm{H}), 6.68(\mathrm{~s}, 1 \mathrm{H}), 2.39(\mathrm{~s}, 3 \mathrm{H}), 1.33(\mathrm{~s}, 9 \mathrm{H}) .{ }^{13} \mathrm{C}\left\{{ }^{1} \mathrm{H}\right\} \mathrm{NMR}\left(101 \mathrm{MHz}, \mathrm{CDCl}_{3}\right) \delta 143.8,140.1$, 137.1, 135.1, 129.6, 127.3, 127.0, 127.0, 125.0, 122.3, 34.3, 30.9, 21.5. HRMS (ESI+) $\mathrm{m} / z$ calcd for $\mathrm{C}_{17} \mathrm{H}_{21} \mathrm{NNaO}_{2} \mathrm{~S}[\mathrm{M}+\mathrm{Na}]^{+}: 326.1185$, found: 326.1182 . IR (neat) 3276, 2963, 1598, 1489, 1444, 1390, 1327, 1215, 1157, 1087, 915, 814, 757, 706, 661, 629, 559, 546, 503, 419.

N-(2-(Tert-butyl)phenyl)methanesulfonamide (1b)<smiles>CC(C)(C)c1ccccc1NS(C)(=O)=O</smiles>

White solid. $75 \%$ yield, $1.7 \mathrm{~g} .{ }^{1} \mathrm{H}$ NMR $\left(400 \mathrm{MHz}, \mathrm{CDCl}_{3}\right) \delta=7.57(\mathrm{dd}, J=8.0,1.4,1 \mathrm{H}), 7.41(\mathrm{dd}, J=8.0,1.6,1 \mathrm{H}), 7.27-7.21(\mathrm{~m}$, $1 \mathrm{H}), 7.13(\mathrm{ddd}, J=7.9,7.3,1.4,1 \mathrm{H}), 6.54(\mathrm{~s}, 1 \mathrm{H}), 3.07(\mathrm{~s}, 3 \mathrm{H}), 1.46(\mathrm{~s}, 9 \mathrm{H}) .{ }^{13} \mathrm{C}\left\{{ }^{1} \mathrm{H}\right\} \mathrm{NMR}\left(101 \mathrm{MHz}, \mathrm{CDCl}_{3}\right) \delta 139.6,135.2,127.4$, 127.2, 125.2, 121.5, 40.2, 34.3, 30.8. HRMS (ESI+) $\mathrm{m} / \mathrm{z}$ calcd for $\mathrm{C}_{11} \mathrm{H}_{17} \mathrm{NNaO}_{2} \mathrm{~S}[\mathrm{M}+\mathrm{Na}]^{+}:$250.0872, found: 250.0872. IR (neat) 3277, $2963,1490,1444,1388,1322,1152,1088,972,912,759,531$

$\mathrm{N}$-(2-(Tert-butyl)-4-nitrophenyl)methanesulfonamide (1c)<smiles>CC(C)(C)c1cc([N+](=O)[O-])ccc1NS(C)(=O)=O</smiles>

Yellow solid. $70 \%$ yield, $0.5 \mathrm{~g} .{ }^{1} \mathrm{H}$ NMR $\left(400 \mathrm{MHz}, \mathrm{CDCl}_{3}\right) \delta=8.37(\mathrm{~d}, J=2.5,1 \mathrm{H}), 7.95(\mathrm{dd}, \mathrm{J}=8.8,2.4,1 \mathrm{H}), 7.58(\mathrm{~d}, J=8.8,1 \mathrm{H})$, $6.68(\mathrm{~s}, 1 \mathrm{H}), 3.19(\mathrm{~s}, 3 \mathrm{H}), 1.50(\mathrm{~s}, 9 \mathrm{H}) .{ }^{13} \mathrm{C}\left\{{ }^{1} \mathrm{H}\right\} \mathrm{NMR}\left(101 \mathrm{MHz}, \mathrm{CDCl}_{3}\right) \delta 146.9,146.5,136.3,128.2,119.4,115.9,41.3,34.9,30.5$. HRMS (ESI+) $\mathrm{m} / \mathrm{z}$ calcd for $\mathrm{C}_{11} \mathrm{H}_{16} \mathrm{~N}_{2} \mathrm{NaO}_{4} \mathrm{~S}[\mathrm{M}+\mathrm{Na}]^{+}:$295.0723, found: 295.0724. IR (neat) 2969, 2361, 1522, 1487, 1393, 1350, 1212, 1155, 1069, 971, 818, 751, 518. 
N-(4-Bromo-2-(tert-butyl)phenyl)methanesulfonamide (1d)<smiles>CC(C)(C)c1cc(Br)ccc1NS(C)(=O)=O</smiles>

White solid. $63 \%$ yield, $1.0 \mathrm{~g} .{ }^{1} \mathrm{H}$ NMR $\left(400 \mathrm{MHz}, \mathrm{CDCl}_{3}\right) \delta=7.51(\mathrm{~d}, J=2.3,1 \mathrm{H}), 7.46(\mathrm{~d}, J=8.6,1 \mathrm{H}), 7.36(\mathrm{dd}, J=8.6,2.3,1 \mathrm{H})$, $6.46(\mathrm{~s}, 1 \mathrm{H}), 3.06(\mathrm{~s}, 3 \mathrm{H}), 1.44(\mathrm{~s}, 9 \mathrm{H}) .{ }^{13} \mathrm{C}\left\{{ }^{1} \mathrm{H}\right\} \mathrm{NMR}\left(101 \mathrm{MHz}, \mathrm{CDCl}_{3}\right) \delta 141.9,134.3,130.5,130.3,123.2,118.6,40.3,34.5,30.6$. HRMS (ESI+) $\mathrm{m} / \mathrm{z}$ calcd for $\mathrm{C}_{11} \mathrm{H}_{16} \mathrm{BrNNaO}_{2} \mathrm{~S}[\mathrm{M}+\mathrm{Na}]^{+}: 327.9977$ and 329.9957 , found: 327.9974 and 329.9962. IR (neat) 3297, 2961, 2358, 1485, 1384, 1306, 1167, 1141, 989, 807, 747, 524, 496, 419.

$\mathrm{N}$-(2-(Tert-butyl)-4-iodophenyl)methanesulfonamide (1e)<smiles>CC(C)(C)c1cc(I)ccc1NS(C)(=O)=O</smiles>

White solid. 83\% yield, $2.9 \mathrm{~g} .{ }^{1} \mathrm{H}$ NMR $\left(400 \mathrm{MHz}, \mathrm{CDCl}_{3}\right) \delta=7.68(\mathrm{~d}, J=2.1,1 \mathrm{H}), 7.55(\mathrm{dd}, J=8.6,2.1,1 \mathrm{H}), 7.33(\mathrm{~d}, J=8.5,1 \mathrm{H})$, $6.48(\mathrm{~s}, 1 \mathrm{H}), 3.06(\mathrm{~s}, 3 \mathrm{H}), 1.43(\mathrm{~s}, 9 \mathrm{H}) .{ }^{13} \mathrm{C}\left\{{ }^{1} \mathrm{H}\right\} \mathrm{NMR}\left(101 \mathrm{MHz}, \mathrm{CDCl}_{3}\right) \delta 141.8,136.4,136.4,135.2,123.1,89.8,40.3,34.3,30.6$. HRMS (ESI+) $\mathrm{m} / \mathrm{z}$ calcd for $\mathrm{C}_{11} \mathrm{H}_{16} \mathrm{INNaO}_{2} \mathrm{~S}[\mathrm{M}+\mathrm{Na}]^{+}: 375.9839$, found: 375.9833 . IR (neat) 2963, 1483, 1386, 1323, 1217, 1151, 1085, $971,916,867,759,699,627,555,518$.

$N$-(3-(Tert-butyl)-[1,1'-biphenyl]-4-yl)methanesulfonamide (1f)<smiles>CC(C)(C)c1cc(-c2ccccc2)ccc1NS(C)(=O)=O</smiles>

White solid. 44\% yield, $0.5 \mathrm{~g} .{ }^{1} \mathrm{H}$ NMR $\left(400 \mathrm{MHz}, \mathrm{CDCl}_{3}\right) \delta=7.68-7.60(\mathrm{~m}, 2 \mathrm{H}), 7.59-7.52(\mathrm{~m}, 2 \mathrm{H}), 7.49-7.41(\mathrm{~m}, 3 \mathrm{H}), 7.39-$ $7.31(\mathrm{~m}, 1 \mathrm{H}), 6.55(\mathrm{~s}, 1 \mathrm{H}), 3.11(\mathrm{~s}, 3 \mathrm{H}), 1.51(\mathrm{~s}, 9 \mathrm{H}) .{ }^{13} \mathrm{C}\left\{{ }^{1} \mathrm{H}\right\}$ NMR $\left(101 \mathrm{MHz}, \mathrm{CDCl}_{3}\right) \delta 140.7,139.9,138.0,134.5,128.8,127.3,127.0$, 126.3, 126.0, 121.9, 40.3, 34.5, 30.9. HRMS (ESI+) $\mathrm{m} / \mathrm{z}$ calcd for $\mathrm{C}_{17} \mathrm{H}_{21} \mathrm{NNaO}_{2} \mathrm{~S}$ [M+Na] $]^{+}$326.1185, found: 326.1181. IR (neat) 3318, 2962, 2359, 2340, 1484, 1385, 1309, 1145, 1086, 973, 921, 758, 697, 522, 418.

$N$-(2-lodo-4,6-dimethylphenyl)methanesulfonamide (1g)<smiles>Cc1cc(C)c(NS(C)(=O)=O)c(I)c1</smiles>

White solid. $50 \%$ yield, $0.4 \mathrm{~g} .{ }^{1} \mathrm{H}$ NMR $\left(400 \mathrm{MHz}, \mathrm{CDCl}_{3}\right) \delta=7.55(\mathrm{~s}, 1 \mathrm{H}), 7.07(\mathrm{~s}, 1 \mathrm{H}), 6.05(\mathrm{~s}, 1 \mathrm{H}), 3.17(\mathrm{~s}, 3 \mathrm{H}), 2.50(\mathrm{~s}, 3 \mathrm{H}), 2.27$ (s, 3H). ${ }^{13} \mathrm{C}\left\{{ }^{1} \mathrm{H}\right\}$ NMR $\left(101 \mathrm{MHz}, \mathrm{CDCl}_{3}\right) \delta 139.9,139.8,137.8,133.3,132.9,100.2,42.7,21.0,20.4$. HRMS (ESI+) $\mathrm{m} / \mathrm{z} \mathrm{calcd}$ for $\mathrm{C}_{9} \mathrm{H}_{12} \mathrm{NNaO}_{2} \mathrm{~S}[\mathrm{M}+\mathrm{Na}]^{+}:$347.9526, found: 3479522. IR (neat) 2361, 2340, 1314, 1276, 1160, 750, 671, 418. 
N-(4-Bromo-2-iodo-6-methylphenyl)methanesulfonamide (1h)<smiles>Cc1cc(Br)cc(I)c1NS(C)(=O)=O</smiles>

White solid. $30 \%$ yield, $0.8 \mathrm{~g} .{ }^{1} \mathrm{H}$ NMR $\left(400 \mathrm{MHz}, \mathrm{CDCl}_{3}\right) \delta=7.87(\mathrm{~d}, J=2.2,1 \mathrm{H}), 7.43(\mathrm{~d}, \mathrm{~J}=2.2,1 \mathrm{H}), 6.00(\mathrm{~s}, 1 \mathrm{H}), 3.19(\mathrm{~s}, 3 \mathrm{H})$, $2.52(\mathrm{~s}, 3 \mathrm{H}) .{ }^{13} \mathrm{C}\left\{{ }^{1} \mathrm{H}\right\}$ NMR $\left(101 \mathrm{MHz}, \mathrm{CDCl}_{3}\right) \delta 141.7,139.3,135.3,134.9,122.4,100.7,43.0,21.0$. HRMS (ESI+) $\mathrm{m} / \mathrm{z} \mathrm{calcd}$ for $\mathrm{C}_{8} \mathrm{H}_{9} \mathrm{BrINNaO}_{2} \mathrm{~S}[\mathrm{M}+\mathrm{Na}]^{+}: 411.8474$ and 413.8454 , found: 411.8470 and 413.8456 . IR (neat) $3260,2360,1456,1314,1166,1135,982$, $858,766,579,521$

N-(2-Bromo-6-methylphenyl)methanesulfonamide (1i)<smiles>Cc1cccc(Br)c1NS(C)(=O)=O</smiles>

Pink solid. 30\% yield, 0.9 g. ${ }^{1} \mathrm{H}$ NMR $\left(400 \mathrm{MHz}, \mathrm{CDCl}_{3}\right) \delta=7.47$ (ddd, $\left.J=8.0,1.4,0.7,1 \mathrm{H}\right), 7.25$ (ddd, $\left.J=8.3,1.9,1.1,1 \mathrm{H}\right), 7.09(\mathrm{t}$, $J=7.8,1 \mathrm{H}), 6.29(\mathrm{~s}, 1 \mathrm{H}), 3.13(\mathrm{~s}, 3 \mathrm{H}), 2.54(\mathrm{~s}, 3 \mathrm{H}) .{ }^{13} \mathrm{C}\left\{{ }^{1} \mathrm{H}\right\} \mathrm{NMR}\left(101 \mathrm{MHz}, \mathrm{CDCl}_{3}\right) \delta 141.2,132.9,131.2,130.7,129.1,123.1,41.8$ 20.4. HRMS (ESI+) $\mathrm{m} / \mathrm{z}$ calcd for $\mathrm{C}_{18} \mathrm{H}_{10} \mathrm{BrNNaO}_{2} \mathrm{~S}[\mathrm{M}+\mathrm{Na}]^{+}: 285.9508$ and 287.9487, found: 285.9504 and 287.9485. IR (neat) 3262, $2359,1460,1379,1316,1261,1162,1131,978,895,768,656,520$.

$N-(2-($ Tert-butyl)phenyl)benzenesulfonamide (1j)<smiles>CC(C)(C)c1ccccc1NS(=O)(=O)c1ccccc1</smiles>

White solid. 86\% yield, 2.5 g. ${ }^{1} \mathrm{H}$ NMR $\left(400 \mathrm{MHz}, \mathrm{CDCl}_{3}\right) \delta=7.87-7.81(\mathrm{~m}, 2 \mathrm{H}), 7.58-7.52(\mathrm{~m}, 1 \mathrm{H}), 7.49-7.40(\mathrm{~m}, 3 \mathrm{H}), 7.31(\mathrm{dd}$, $J=7.8,1.7,1 \mathrm{H}), 7.10(\mathrm{dtd}, J=24.7,7.4,1.6,2 \mathrm{H}), 6.69(\mathrm{~s}, 1 \mathrm{H}), 1.32(\mathrm{~s}, 9 \mathrm{H}) .{ }^{13} \mathrm{C}\left\{{ }^{1} \mathrm{H}\right\} \mathrm{NMR}\left(101 \mathrm{MHz}, \mathrm{CDCl}_{3}\right) \delta 140.4,140.1,134.9$, 133.0, 129.0, 127.2, 127.1, 127.0, 125.3, 122.7, 34.3, 30.9. HRMS (ESI+) $\mathrm{m} / \mathrm{z}$ calcd for $\mathrm{C}_{16} \mathrm{H}_{19} \mathrm{NNaO}_{2} \mathrm{~S}[\mathrm{M}+\mathrm{Na}]^{+}: 312.1029$, found: 312.1023. IR (neat) 3278, 2964, 2359, 1488, 1447, 1390, 1327, 1159, 1088, 918, 756, 717, 689, 632, 588, 559.

N-(2-(Tert-butyl)phenyl)-1-phenylmethanesulfonamide (1k)<smiles>CC(C)(C)c1ccccc1NS(=O)(=O)Cc1ccccc1</smiles>

White solid. $36 \%$ yield, $1.1 \mathrm{~g} .{ }^{1} \mathrm{H}$ NMR $\left(400 \mathrm{MHz}, \mathrm{CDCl}_{3}\right) \delta=7.47(\mathrm{dd}, J=8.1,1.4,1 \mathrm{H}), 7.38-7.26(\mathrm{~m}, 6 \mathrm{H}), 7.26-7.20(\mathrm{~m}, 1 \mathrm{H})$, $7.09(\mathrm{td}, J=7.6,1.4,1 \mathrm{H}), 6.30(\mathrm{~s}, 1 \mathrm{H}), 4.48(\mathrm{~s}, 2 \mathrm{H}), 1.29(\mathrm{~s}, 9 \mathrm{H}) .{ }^{13} \mathrm{C}\left\{{ }^{1} \mathrm{H}\right\}$ NMR $\left(101 \mathrm{MHz}, \mathrm{CDCl}_{3}\right) \delta 138.2,135.5,130.7,128.9,128.8$, 128.4, 127.3, 127.3, 124.3, 119.7, 58.5, 34.1, 30.5. HRMS (ESI+) $\mathrm{m} / \mathrm{z}$ calcd for $\mathrm{C}_{17} \mathrm{H}_{21} \mathrm{NNaO}_{2} \mathrm{~S}[\mathrm{M}+\mathrm{Na}]^{+}: 326.1185$, found: 326.1181 IR (neat) 3430, 2966, 2359, 1493, 1446, 1394, 1334, 1274, 1201, 1153, 1130, 1087, 924, 839, 757, 697, 606, 540. 
N-(2-(Tert-butyl)phenyl)cyclohexanesulfonamide (1I)

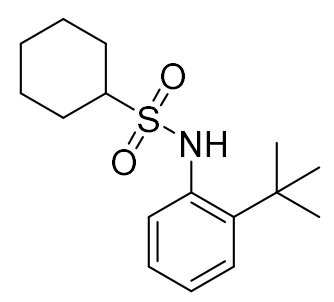

White solid. 23\% yield, 0.2 g. ${ }^{1} \mathrm{H}$ NMR $\left(400 \mathrm{MHz}, \mathrm{CDCl}_{3}\right) \delta=7.41$ (ddd, $\left.J=18.8,8.0,1.5,2 \mathrm{H}\right), 7.20$ (td, $\left.J=7.8,1.6,1 \mathrm{H}\right), 7.06(\mathrm{ddd}$, $J=8.0,7.3,1.4,1 \mathrm{H}), 6.31(\mathrm{~s}, 1 \mathrm{H}), 3.22(\mathrm{tt}, J=12.1,3.5,1 \mathrm{H}), 2.30-2.12(\mathrm{~m}, 2 \mathrm{H}), 1.90(\mathrm{dt}, J=10.0,2.8,2 \mathrm{H}), 1.75-1.61(\mathrm{~m}, 3 \mathrm{H})$, $1.47(\mathrm{~s}, 9 \mathrm{H}), 1.31-1.22(\mathrm{~m}, 3 \mathrm{H}) .{ }^{13} \mathrm{C}\left\{{ }^{1} \mathrm{H}\right\} \mathrm{NMR}\left(101 \mathrm{MHz}, \mathrm{CDCl}_{3}\right) \delta 138.3,135.8,127.1,127.1,124.0,120.2,62.0,34.2,30.7,26.3$, 25.1, 25.0. HRMS (ESI+) $\mathrm{m} / \mathrm{z}$ calcd for $\mathrm{C}_{16} \mathrm{H}_{25} \mathrm{NNaO}_{2} \mathrm{~S}[\mathrm{M}+\mathrm{H}]^{+}:$318.1498, found: 318.1494. IR (neat) 2938, 2859, 2360, 1492, 1448, 1392, 1325, 1275, 1215, 1146, 1087, 914, 753, 635, 597, 545. 


\subsection{General procedures for the preparation of $\alpha, \beta$-unsaturated carbonic anhydride 2}

Anhydrides $\mathbf{2 a}, \mathbf{2 b}, \mathbf{2 c}, \mathbf{2 d}, \mathbf{2 g}, \mathbf{2 j}, \mathbf{2 n}, \mathbf{2 0}, \mathbf{2 p}$, and $\mathbf{2 q}$ were known compounds and synthesized according to the reported procedures. ${ }^{2}$ Anhydrides $2 \mathbf{e}, \mathbf{2} \mathbf{f}, \mathbf{2} \mathbf{h}, \mathbf{2} \mathbf{i}, \mathbf{2 k}, \mathbf{2} \mathbf{l}$ and $\mathbf{2 m}$ were synthesized according to the following procedure:

General procedure for preparation of $\mathbf{2}$ :<smiles>[R]C=C([R2])C(=O)O[CH+]C(=O)OC(C)C(=O)OC(C)C(=O)OC(C)C</smiles>

In a round-bottom flask, substituted acrylic acids (10.0 mmol, 1.0 equiv) and trimethylamine (1.4 mL, $10.0 \mathrm{mmol}, 1.0$ equiv) were dissolved in $50 \mathrm{~mL}$ anhydrous tetrahydrofuran. The mixture was stirred for $10 \mathrm{~min}$ at room temperature. In another round-bottom flask, a $2 \mathrm{M}$ isopropyl choloroformate toluene solution $(6.0 \mathrm{~mL}, 12.0 \mathrm{mmol}, 1.2$ equiv) was diluted with $50 \mathrm{~mL}$ anhydrous tetrahydrofuran. At $0{ }^{\circ} \mathrm{C}$ in an ice/water bath, the mixture of acrylic acid and triethylamine was added dropwise to the diluted isopropyl choloroformate solution via addition funnel over $40 \mathrm{~min}$. Then, the reaction mixture was stirred at room temperature for additional $1 \mathrm{hour}$ and monitored by TLC. When TLC showed full conversion, $10 \%$ citric acid $(30 \mathrm{~mL})$ was added to the reaction mixture until the system became clear. After extraction with ethyl ether $(40 \mathrm{~mL} \times 2)$, the organic layer was washed with saturated sodium bicarbonate solution ( $40 \mathrm{~mL})$ and brine $(40 \mathrm{~mL})$, dried over $\mathrm{MgSO}_{4}$, and concentrated in vacuo. Purification by flash silica gel column chromatography using hexane/EtOAc $(v / v=10 / 1)$ as an eluent gave the corresponding carbonic anhydride.

(E)-3-(4-Fluorophenyl)acrylic (isopropyl carbonic) anhydride (2e)<smiles>CC(C)OC(=O)OC(=O)/C=C/c1ccc(F)cc1</smiles>

Colorless oil. 90\% yield, $2.5 \mathrm{~g} .{ }^{1} \mathrm{H}$ NMR $\left(400 \mathrm{MHz}, \mathrm{CDCl}_{3}\right) \delta=7.78(\mathrm{~d}, J=15.9,1 \mathrm{H}), 7.61-7.50(\mathrm{~m}, 2 \mathrm{H}), 7.11(\mathrm{dd}, J=9.6,7.6,2 \mathrm{H})$, $6.39-6.29(\mathrm{~m}, 1 \mathrm{H}), 5.05$ (hept, $J=6.3,1 \mathrm{H}), 1.39(\mathrm{~d}, J=6.3,6 \mathrm{H}) .{ }^{13} \mathrm{C}\left\{{ }^{1} \mathrm{H}\right\}$ NMR $\left(101 \mathrm{MHz}, \mathrm{CDCl}_{3}\right) \delta 165.48(\mathrm{~d}, J=254.5 \mathrm{~Hz}), 161.0$, 148.6, 147.5, 130.5 (d, $J=8.1 \mathrm{~Hz}), 129.9$ (d, $J=3.0 \mathrm{~Hz}), 116.3(\mathrm{~d}, J=22.2 \mathrm{~Hz}), 115.3$ (d, $J=3.0 \mathrm{~Hz}), 74.3,21.5 .{ }^{19} \mathrm{~F}\left\{{ }^{1} \mathrm{H}\right\} \mathrm{NMR}(376$ $\left.\mathrm{MHz}, \mathrm{CDCl}_{3}\right) \delta$. 107.6. HRMS (ESI+) $\mathrm{m} / \mathrm{z}$ calcd for $\mathrm{C}_{13} \mathrm{H}_{13} \mathrm{FNaO}_{4}[\mathrm{M}+\mathrm{Na}]^{+}:$275.0690, found: 275.0687. IR (neat) 2986, 1794, 1732, $1632,1598,1509,1468,1416,1378,1234,1153,1063,982,901,829,749,510,473$

(E)-3-(4-Chlorophenyl)acrylic (isopropyl carbonic) anhydride (2f)<smiles>CC(C)OC(=O)OC(=O)/C=C/c1ccc(Cl)cc1</smiles>

White solid. $52 \%$ yield, $1.4 \mathrm{~g} .{ }^{1} \mathrm{H}$ NMR $\left(400 \mathrm{MHz}, \mathrm{CDCl}_{3}\right) \delta=7.76(\mathrm{~d}, J=16.0,1 \mathrm{H}), 7.47(\mathrm{~d}, J=8.5,2 \mathrm{H}), 7.38(\mathrm{~d}, J=8.5,2 \mathrm{H}), 6.39$ $(\mathrm{d}, J=16.0,1 \mathrm{H}), 5.04$ (hept, $J=6.3,1 \mathrm{H}), 1.38(\mathrm{~d}, J=6.3,6 \mathrm{H}) .{ }^{13} \mathrm{C}\left\{{ }^{1} \mathrm{H}\right\}$ NMR $\left(101 \mathrm{MHz}, \mathrm{CDCl}_{3}\right) \delta 160.9,148.5,147.3,137.3,132.0$, 129.6, 129.4, 116.1, 74.4, 21.5. HRMS (ESI+) $\mathrm{m} / \mathrm{z}$ calcd for $\mathrm{C}_{13} \mathrm{H}_{13} \mathrm{CINaO}_{4}[\mathrm{M}+\mathrm{Na}]^{+}: 291.0395$ and 293.0365, found: 291.0390 and 293.0357. IR (neat) 2984, 2360, 1786, 1728, 1633, 1492, 1375, 1277, 1254, 1127, 1098, 1069, 999, 910, 821, $750,498$.

(E)-3-(3-Bromophenyl)acrylic (isopropyl carbonic) anhydride (2h)<smiles>CC(C)OC(=O)OC(=O)/C=C/c1cccc(Br)c1</smiles>

Colorless oil. $48 \%$ yield, $1.5 \mathrm{~g} .{ }^{1} \mathrm{H}$ NMR $\left(400 \mathrm{MHz}, \mathrm{CDCl}_{3}\right) \delta=7.73(\mathrm{~d}, \mathrm{~J}=16.0,1 \mathrm{H}), 7.68(\mathrm{t}, \mathrm{J}=1.8,1 \mathrm{H}), 7.58-7.53(\mathrm{~m}, 1 \mathrm{H}), 7.46$ (dt, $J=7.8,1.2,1 \mathrm{H}), 7.29(\mathrm{t}, J=7.8,1 \mathrm{H}), 6.41(\mathrm{~d}, J=16.0,1 \mathrm{H}), 5.05$ (hept, $J=6.3,1 \mathrm{H}), 1.39(\mathrm{~d}, J=6.3,6 \mathrm{H}) .{ }^{13} \mathrm{C}\left\{{ }^{1} \mathrm{H}\right\} \mathrm{NMR}(101 \mathrm{MHz}$,

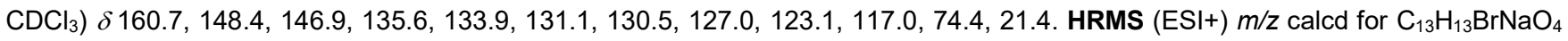
$[\mathrm{M}+\mathrm{Na}]^{+}: 334.9889$ and 336.9869 , found: 334.9883 and 336.9862. IR (neat) 2986, 2360, 1798, 1737, 1633, 1563, 1470, 1240, 1157, $1122,1067,902,750,668$. 
(E)-3-(2-Bromophenyl)acrylic (isopropyl carbonic) anhydride (2i)<smiles>CC(C)OC(=O)OC(=O)/C=C/c1ccccc1Br</smiles>

Colorless oil. $58 \%$ yield, $1.8 \mathrm{~g} .{ }^{1} \mathrm{H}$ NMR $\left(400 \mathrm{MHz}, \mathrm{CDCl}_{3}\right) \delta=8.19(\mathrm{~d}, J=15.9,1 \mathrm{H}), 7.63(\mathrm{ddd}, J=9.1,7.9,1.5,2 \mathrm{H}), 7.41-7.24(\mathrm{~m}$, $2 \mathrm{H}), 6.38(\mathrm{~d}, J=15.9,1 \mathrm{H}), 5.06(\mathrm{hept}, J=6.3,1 \mathrm{H}), 1.40(\mathrm{~d}, J=6.3,6 \mathrm{H}) .{ }^{13} \mathrm{C}\left\{{ }^{1} \mathrm{H}\right\} \mathrm{NMR}\left(101 \mathrm{MHz}, \mathrm{CDCl}_{3}\right) \delta 160.7,148.6,147.0,133.7$, 133.6, 132.1, 128.0, 127.8, 125.8, 118.3, 74.5, 21.5. HRMS (ESI+) $\mathrm{m} / \mathrm{z}$ calcd for $\mathrm{C}_{13} \mathrm{H}_{13} \mathrm{BrNaO}_{4}[\mathrm{M}+\mathrm{Na}]^{+}: 334.9889$ and 336.9869 , found: 334.9885 and 336.9863. IR (neat) 2987, 2360, 1799, 1738, 1629, 1276, 1161, 1075, 751.

(E)-3-(4-Cyanophenyl)acrylic (isopropyl carbonic) anhydride (2k)<smiles>CC(C)OC(=O)OC(=O)/C=C/c1ccc(C#N)cc1</smiles>

White solid. 39\% yield, $0.5 \mathrm{~g} .{ }^{1} \mathrm{H}$ NMR $\left(400 \mathrm{MHz}, \mathrm{CDCl}_{3}\right) \delta=7.80(\mathrm{~d}, \mathrm{~J}=16.1,1 \mathrm{H}), 7.71(\mathrm{~d}, \mathrm{~J}=8.3,2 \mathrm{H}), 7.64(\mathrm{~d}, J=8.4,2 \mathrm{H}), 6.50$ $(\mathrm{d}, J=16.0,1 \mathrm{H}), 5.06$ (hept, $J=6.3,1 \mathrm{H}), 1.40(\mathrm{~d}, J=6.3,6 \mathrm{H}) .{ }^{13} \mathrm{C}\left\{{ }^{1} \mathrm{H}\right\}$ NMR $\left(101 \mathrm{MHz}, \mathrm{CDCl}_{3}\right) \delta 160.4,148.3,146.0,137.7,132.8$, 128.8, 119.2, 118.1, 114.3, 74.7, 21.5. HRMS (ESI+) $\mathrm{m} / \mathrm{z}$ calcd for $\mathrm{C}_{14} \mathrm{H}_{13} \mathrm{NNaO}_{4}[\mathrm{M}+\mathrm{Na}]^{+}:$: 282.0737, found: 282.0736. IR (neat) 2361, $1795,1276,1095,750$.

(E)-(4-Nitrophenyl)acrylic (isopropyl carbonic) anhydride (2I)<smiles>CC(C)OC(=O)OC(=O)/C=C/c1ccc([N+](=O)[O-])cc1</smiles>

Yellow solid. $54 \%$ yield, $1.5 \mathrm{~g} .{ }^{1} \mathrm{H}$ NMR $\left(400 \mathrm{MHz}, \mathrm{CDCl}_{3}\right) \delta=8.27(\mathrm{~d}, J=8.7,2 \mathrm{H}), 7.84(\mathrm{dt}, J=16.1,0.5,1 \mathrm{H}), 7.70(\mathrm{dd}, J=8.9,0.6$, $2 \mathrm{H}), 6.54(\mathrm{~d}, J=16.0,1 \mathrm{H}), 5.05$ (hept, $J=6.3,1 \mathrm{H}), 1.39(\mathrm{~d}, J=6.3,6 \mathrm{H}) .{ }^{13} \mathrm{C}\left\{{ }^{1} \mathrm{H}\right\} \mathrm{NMR}\left(101 \mathrm{MHz}, \mathrm{CDCl}_{3}\right) \delta .160 .3,149.0,148.2,145.5$, 139.4, 129.1, 124.3, 119.8, 74.7, 21.5. HRMS (ESI+) $\mathrm{m} / \mathrm{z}$ calcd for $\mathrm{C}_{13} \mathrm{H}_{13} \mathrm{NNaO}_{6}[\mathrm{M}+\mathrm{Na}]^{+}:$302.0635, found: 302.0633 . IR (neat) 2359, $1794,1736,1640,1602,1522,1348,1253,1132,1095,1002,901,842,752$.

(E)-3-(Naphthalen-1-yl) (Isopropyl carbonic) acrylic anhydride (2m):<smiles>CC(C)OC(=O)OC(=O)/C=C/c1cccc2ccccc12</smiles>

Yellow oil. $67 \%$ yield, 1.9 g. ${ }^{1} \mathrm{H}$ NMR $\left(400 \mathrm{MHz}, \mathrm{CDCl}_{3}\right) \delta=8.67(\mathrm{~d}, J=15.7,1 \mathrm{H}), 8.18(\mathrm{~d}, J=8.3,1 \mathrm{H}), 7.92(\mathrm{dd}, J=21.5,8.1,2 \mathrm{H})$, $7.79(\mathrm{~d}, J=7.2,1 \mathrm{H}), 7.66-7.46(\mathrm{~m}, 3 \mathrm{H}), 6.53(\mathrm{~d}, J=15.7,1 \mathrm{H}), 5.09$ (hept, $J=6.2,1 \mathrm{H}), 1.42(\mathrm{~d}, J=6.3,6 \mathrm{H}) .{ }^{13} \mathrm{C}\left\{{ }^{1} \mathrm{H}\right\} \mathrm{NMR}(101 \mathrm{MHz}$, $\left.\mathrm{CDCl}_{3}\right) \delta 161.1,148.7,145.8,133.7,131.6,131.3,130.8,128.8,127.2,126.4,125.6,125.4,123.1,117.9,74.4,21.5$. HRMS (ESI+) $\mathrm{m} / \mathrm{z}$ calcd for $\mathrm{C}_{17} \mathrm{H}_{16} \mathrm{NaO}_{4}[\mathrm{M}+\mathrm{Na}]^{+}:$307.0941, found: 307.0937. IR (neat) 2986, 2360, 1794, 1732, 1629, 1467, 1348, 1261, 1234, 1156, 1122, 1093, 1067, 977, 901, 797, 750, 593. 


\section{General procedures for the preparation of racemic products}

Representative experimental procedure for the reaction of sulfonamide $1 \mathbf{a}$ and carbonic anhydride $\mathbf{2 a}$

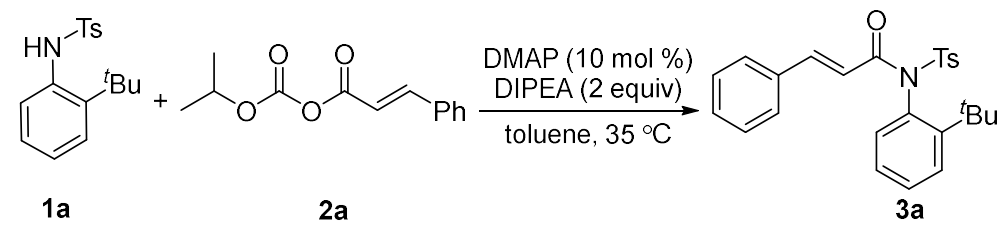

DMAP $(10 \mathrm{~mol} \%, 1.22 \mathrm{mg})$, and sulfonamide $1 \mathrm{a}(0.1 \mathrm{mmol}, 22.7 \mathrm{mg})$ were stirred in toluene $(1.0 \mathrm{~mL})$ at $35^{\circ} \mathrm{C}$ in a water bath. Then DIPEA $(0.2 \mathrm{mmol}, 33 \mu \mathrm{L}), \alpha, \beta$-unsaturated carbonic anhydride $2 \mathrm{a}(0.15 \mathrm{mmol}, 35.1 \mathrm{mg})$ was added. The mixture was stirred at $35{ }^{\circ} \mathrm{C}$ for $24 \mathrm{~h}$. Then the reaction mixture was subjected to column chromatography on silica gel and eluted with petroleum ether and acetone $(\mathrm{v} / \mathrm{v}, 10: 1)$ to afford product $3 \mathrm{a}$ as a white solid.

\section{General procedures for the catalytic asymmetric reaction}

Representative experimental procedure for the reaction of sulfonamide $\mathbf{1 a}$ and carbonic anhydride $\mathbf{2 a}$

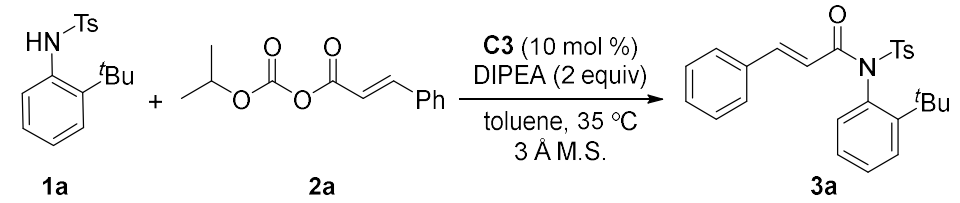

Under an atmosphere of nitrogen, $(R)$-HBTM C3 (10 mol \%, $2.66 \mathrm{mg}), 3 \AA$ M.S. (15 mg) and sulfonamide $1 \mathrm{a}(0.1 \mathrm{mmol}, 22.7 \mathrm{mg})$ were stirred in toluene $(1.0 \mathrm{~mL})$ at $35^{\circ} \mathrm{C}$ in a water bath. Then DIPEA $(0.2 \mathrm{mmol}, 33 \mu \mathrm{L}), \alpha, \beta$-unsaturated carbonic anhydride $2 \mathrm{a}(0.15$ $\mathrm{mmol}, 35.1 \mathrm{mg}$ ) was added. The mixture was stirred at $35{ }^{\circ} \mathrm{C}$ for $24 \mathrm{~h}$. Then the reaction mixture was subjected to column chromatography on silica gel and eluted with petroleum ether and acetone (v/v, 10:1) to afford $3 \mathrm{a}$ as a white solid. 


\section{Optimization of the reaction conditions}

Table S1. The screening of catalysts.<smiles>[NH3+]c1ccccc1Br</smiles>

1a<smiles>CC(=O)OC(=O)OC(C)C</smiles>

$2 a$<smiles>CC(C)(C)c1ccccc1N([18F])C(=O)/C=C/c1ccccc1</smiles>

3a

C1

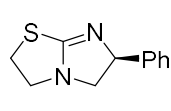

C2

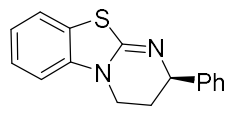

C3

\begin{tabular}{|c|c|c|c|c|}
\hline entry ${ }^{a}$ & cat. & base & yield $^{b}(\%)$ & $\mathrm{ee}^{c}(\%)$ \\
\hline 1 & C1 & - & NR & - \\
\hline 2 & C2 & - & NR & - \\
\hline 3 & C3 & - & trace & - \\
\hline 4 & C1 & $\mathrm{KO}^{t} \mathrm{Bu}$ (2 equiv) & trace & - \\
\hline 5 & C2 & $\mathrm{KO} \mathrm{B}^{t} \mathrm{Bu}$ (2 equiv) & NR & - \\
\hline 6 & C3 & $\mathrm{KO} \mathrm{B}^{t} \mathrm{Bu}$ (2 equiv) & - & 60 \\
\hline $7^{d}$ & C3 & $\mathrm{KO} \mathrm{K}^{t} \mathrm{Bu}$ (2 equiv) & 96 & 67 \\
\hline
\end{tabular}

Table S2. The screening of solvents.

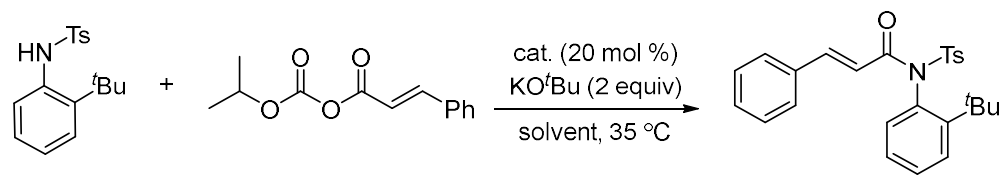

$1 \mathrm{a}$
$2 a$

$$
3 a
$$

\begin{tabular}{lllll}
\hline entry $^{a}$ & solvent & cat. & yield $(\%)$ & ee $^{c}(\%)$ \\
\hline 1 & THF & C3 & 72 & 35 \\
2 & Toluene & C3 & 80 & 81 \\
3 & $\mathrm{Et}_{2} \mathrm{O}$ & $\mathbf{C 3}$ & 77 & 57 \\
4 & $\mathrm{DCM}$ & $\mathbf{C 3}$ & 96 & 63 \\
5 & $\mathrm{CH}_{3} \mathrm{CN}$ & $\mathbf{C 3}$ & trace & race \\
6 & $\mathrm{DMSO}$ & $\mathbf{C 3}$ & trace & race \\
7 & chlorobenzene & C3 & quant. & 75 \\
8 & bromobenzene & C3 & quant. & 73 \\
9 & DMF & C3 & trace & race \\
10 & EtOAc & C3 & mass & 9 \\
\hline
\end{tabular}

${ }^{a}$ Unless otherwise noted, all reactions were performed with catalyst (20 mol \%), KOtBu (2 equiv), 1a (0.10 $\mathrm{mmol}), 2 \mathrm{a}(0.30 \mathrm{mmol})$ in solvent $(1.0 \mathrm{~mL})$ at $35^{\circ} \mathrm{C}$ for $24 \mathrm{~h}^{b}$ Yield of isolated product. ${ }^{c}$ Determined by HPLC analysis on a chiral stationary phase. 
Table S3. The screening of bases.

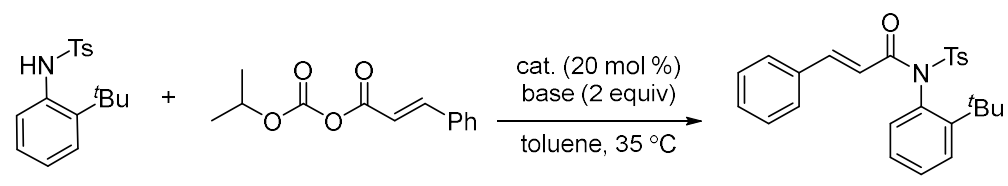

1a

$2 a$

$3 a$

\begin{tabular}{|c|c|c|c|c|}
\hline entry $^{a}$ & base & cat. & yield $b(\%)$ & $\mathrm{ee}^{c}(\%)$ \\
\hline 1 & - & $\mathrm{C} 3$ & 62 & 87 \\
\hline 2 & $\mathrm{KO}^{t} \mathrm{Bu}$ & C3 & 80 & 81 \\
\hline 3 & $\mathrm{NaO}^{t} \mathrm{Bu}$ & C3 & quant. & 60 \\
\hline 4 & $\mathrm{LiO}^{t} \mathrm{Bu}$ & C3 & quant. & race \\
\hline 5 & EtONa & C3 & 80 & 67 \\
\hline 6 & KOMe & C3 & 77 & 53 \\
\hline 7 & $\mathrm{NaOMe}$ & C3 & 38 & 65 \\
\hline 8 & $\mathrm{DABCO}$ & $\mathrm{C} 3$ & trace & 51 \\
\hline 9 & DIPEA & C3 & 82 & 87 \\
\hline 10 & $\mathrm{Et}_{3} \mathrm{~N}$ & C3 & 75 & 87 \\
\hline 11 & ${ }^{i} \mathrm{Pr}_{2} \mathrm{NH}$ & $\mathrm{C} 3$ & trace & - \\
\hline 12 & $\mathrm{Et}_{2} \mathrm{NH}$ & C3 & trace & - \\
\hline
\end{tabular}

Table S4. The screening of additives.

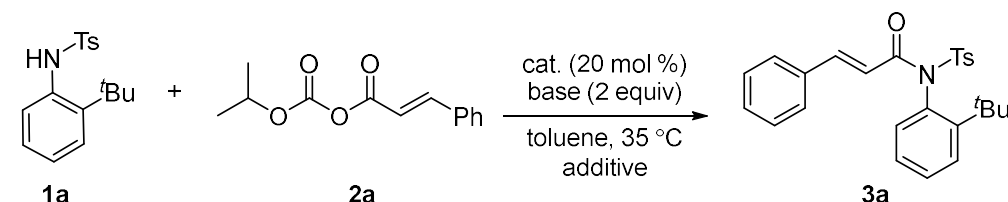

\begin{tabular}{|c|c|c|c|c|}
\hline entry ${ }^{a}$ & additive & cat. & yield $^{b}(\%)$ & $\mathrm{ee}^{c}(\%)$ \\
\hline 1 & - & C3 & 82 & 87 \\
\hline 2 & $3 \AA$ M.S. $(10 \mathrm{mg})$ & C3 & 91 & 87 \\
\hline 3 & $4 \AA$ M.S. $(10 \mathrm{mg})$ & C3 & 65 & 87 \\
\hline 4 & $5 \AA$ M.S. $(10 \mathrm{mg})$ & C3 & NR & - \\
\hline 5 & LiNTf $_{2}(10$ mg) & C3 & 80 & 7 \\
\hline 6 & $\mathrm{LiCl}(10 \mathrm{mg})$ & C3 & 85 & 17 \\
\hline 7 & $\mathrm{NaBArF}_{4}(10 \mathrm{mg})$ & C3 & 75 & 67 \\
\hline
\end{tabular}

${ }^{a}$ Unless otherwise noted, all reactions were performed with catalyst $(20 \mathrm{~mol} \%)$, DIPEA (2 equiv), additive, $1 \mathrm{a}(0.10 \mathrm{mmol}), 2 \mathrm{a}(0.30 \mathrm{mmol})$ in toluene $(1.0 \mathrm{~mL})$ at $35^{\circ} \mathrm{C}$ for $24 \mathrm{~h}^{\mathrm{b}}$ Yield of isolated product. ${ }^{\circ}$ Determined by HPLC analysis on a chiral stationary phase.

Table S5. Optimization of condition for substrate ratio.

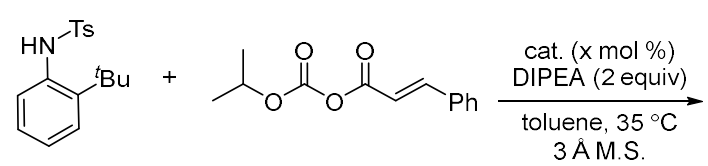

$1 \mathrm{a}$

$2 a$<smiles>[3H]N(C(=O)/C=C/c1ccccc1)c1ccccc1C(C)(C)C</smiles>

$3 a$

\begin{tabular}{lllll}
\hline entry $^{a}$ & $\mathrm{x}$ & cat. & yield $^{b}(\%)$ & $\mathrm{ee}^{c}(\%)$ \\
\hline 1 & 15 & C3 & 91 & 87 \\
2 & 10 & C3 & 91 & 87 \\
3 & 5 & C3 & 89 & 87 \\
4 & 1 & C3 & trace & 83 \\
\hline${ }^{a}$ Unless otherwise noted, all reactions were performed with catalyst, DIPEA (2 equiv), 3 $\AA$ M.S. (10 \\
mg), 1a $\left(0.10\right.$ mmol), 2a $(0.30 \mathrm{mmol})$ in toluene $(1 \mathrm{~mL})$ at $35^{\circ} \mathrm{C}$ for 24 h. ${ }^{b}$ Yield of isolated product. \\
${ }^{c}$ Determined by HPLC analysis on a chiral stationary phase.
\end{tabular}


Table S6. N-protecting group effect.

Cns
entry

Table S7. Optimization of condition for substrate ratio

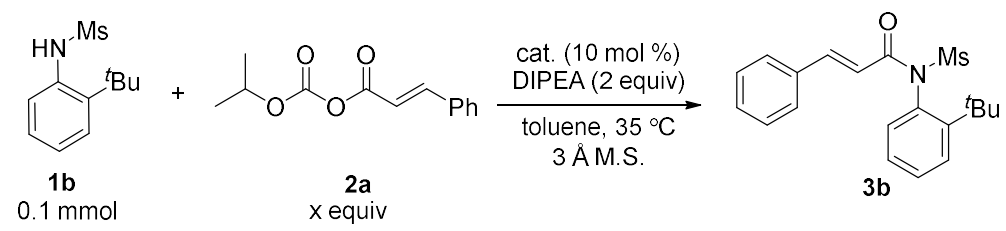

\begin{tabular}{|c|c|c|c|c|}
\hline entry ${ }^{a}$ & $x$ & cat. & yield $^{b}(\%)$ & $\mathrm{ee}^{c}(\%)$ \\
\hline 1 & 3.0 & C3 & 56 & 95 \\
\hline 2 & 2.5 & C3 & 54 & 95 \\
\hline 3 & 2.0 & C3 & 56 & 95 \\
\hline 4 & 1.5 & C3 & 79 & 95 \\
\hline 5 & 1.0 & C3 & 68 & 95 \\
\hline $6^{d}$ & 1.5 & C3 & 88 & 95 \\
\hline
\end{tabular}

Table S8. Leaving group effect.

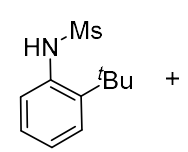

$1 \mathrm{a}$

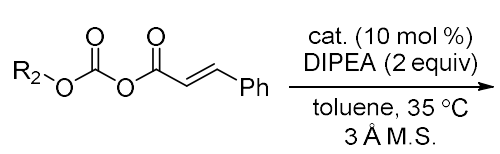

2

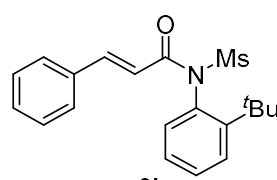

$3 b$

\begin{tabular}{lllll}
\hline entry $^{a}$ & $\mathbf{R}_{\mathbf{2}}$ & cat. & yield $^{b}(\%)$ & ee $^{c}(\%)$ \\
\hline 1 & & & & 95 \\
2 & i Pr & C3 & 88 & 94 \\
3 & Et & C3 & trace & 91 \\
\hline
\end{tabular}

${ }^{a}$ Unless otherwise noted, all reactions were performed with catalyst (10 mol \%), DIPEA (2 equiv), 3 Å M.S. (15 mg), $1(0.10 \mathrm{mmol}), 2 \mathrm{a}(0.15 \mathrm{mmol})$ in toluene $(1 \mathrm{~mL})$ at $35^{\circ} \mathrm{C}$ for $24 \mathrm{~h} .{ }^{b}$ Yield of isolated product. ${ }^{c}$ Determined by HPLC analysis on a chiral stationary phase. 
Table S9. Temperature effect

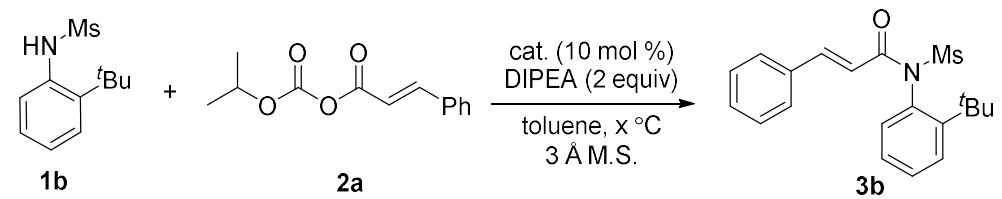

\begin{tabular}{|c|c|c|c|c|}
\hline entry ${ }^{a}$ & $x$ & cat. & yield $^{b}(\%)$ & $\mathrm{ee}^{c}(\%)$ \\
\hline 1 & 20 & C3 & 73 & 94 \\
\hline 2 & 25 & C3 & 75 & 95 \\
\hline 3 & 30 & C3 & 78 & 95 \\
\hline 4 & 35 & C3 & 85 & 95 \\
\hline 5 & 40 & C3 & 80 & 94 \\
\hline $6^{d}$ & 40 & C3 & 67 & 43 \\
\hline
\end{tabular}

${ }^{a}$ Unless otherwise noted, all reactions were performed with catalyst (10 mol \%), DIPEA (2 equiv), $3 \AA$ M.S. (15 mg), $1 \mathbf{b}(0.10 \mathrm{mmol}), 2 \mathbf{a}(0.15 \mathrm{mmol})$ in toluene $(1 \mathrm{~mL})$ at $35^{\circ} \mathrm{C}$ for $24 \mathrm{~h} .{ }^{b}$ Yield of isolated product. ${ }^{c}$ Determined by HPLC analysis on a chiral stationary phase. ${ }^{d}$ Conducted with $1 \mathrm{a}$ $(0.1 \mathrm{mmol})$

Table S10. The screening of catalysts for $\mathbf{1 i}$.<smiles>Cc1cccc(Br)c1NS(C)(=O)=O</smiles>

$1 \mathrm{i}$<smiles>CC(C)OC(=O)OC(=O)/C=C/P</smiles>

2a

$$
\begin{aligned}
& \text { Cat. }(10 \text { mol \%) } \\
& \text { DIPEA ( } 2 \text { equiv) } \\
& \text { toluene } 35^{\circ} \mathrm{C}
\end{aligned}
$$
$3 \AA$ M.S.

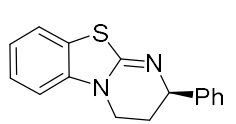<smiles></smiles><smiles>Cc1cccc(Br)c1N(C(=O)/C=C/c1ccccc1)C(C)(C)C</smiles>

$3 y$

C3<smiles>CCC[GeH3]</smiles>

\begin{tabular}{lllll}
\hline entry $^{a}$ & cat. & base & yield $^{b}(\%)$ & $\operatorname{ee}^{c}(\%)$ \\
\hline 1 & C3 & DIPEA & 85 & 75 \\
2 & C4 & DIPEA & 90 & -75 \\
\hline
\end{tabular}

${ }^{a}$ Unless otherwise noted, all reactions were performed catalyst ( $10 \mathrm{~mol} \%$ ), DIPEA (2 equiv), $3 \AA$ M.S. (15 $\mathrm{mg}), \mathbf{1 i}(0.10 \mathrm{mmol}), \mathbf{2 a}(0.15 \mathrm{mmol})$ in toluene $(1 \mathrm{~mL})$ at $35{ }^{\circ} \mathrm{C}$ for $24 \mathrm{~h} .{ }^{b}$ Yield of isolated product. Determined by HPLC analysis on a chiral stationary phase. 


\section{Scope limitation}

\section{1 low reactivity or no reaction}<smiles>O=C(/C=C/c1ccccc1)Oc1ccc([N+](=O)[O-])cc1</smiles>

NR<smiles>O=C(/C=C/c1ccccc1)OC(=O)/C=C/c1ccccc1</smiles>

NR<smiles>O=C(OC(=O)c1ccccc1)c1ccccc1</smiles>

NR

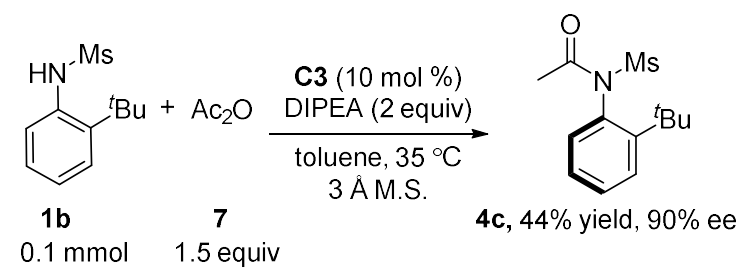

\subsection{Synthesis and characterization of the product $\mathbf{4 a}$ and $\mathbf{4 b}$}

Under an atmosphere of nitrogen, $(R)$-HBTM C3 $(10 \mathrm{~mol} \%, 2.66 \mathrm{mg}), 3 \AA$ M.S. (15 mg) and sulfonamide $1 \mathrm{a}(0.1 \mathrm{mmol}, 22.7 \mathrm{mg})$ were stirred in toluene $(1.0 \mathrm{~mL})$ at $35^{\circ} \mathrm{C}$ in a water bath. Then DIPEA $(0.2 \mathrm{mmol}, 33 \mu \mathrm{L})$, benzoic acid-derived anhydride $6(0.30 \mathrm{mmol}$, $62.4 \mathrm{mg}$ ) was added. The mixture was stirred at $35^{\circ} \mathrm{C}$ for $24 \mathrm{~h}$. Then the reaction mixture was subjected to column chromatography on silica gel and eluted with petroleum ether and acetone $(v / v, 10: 1)$ to afford $4 a$.

\begin{tabular}{l} 
entry ${ }^{a}$ \\
\hline 1 \\
2
\end{tabular}

\subsection{Commercially available starting materials version of the asymmetric reaction}

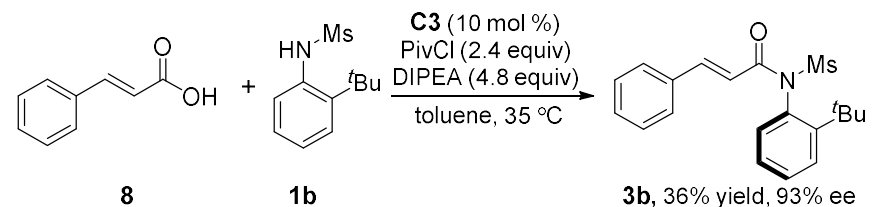

Under an atmosphere of nitrogen, $(R)$-HBTM C3 (10 mol \%, $2.66 \mathrm{mg})$, cinnamic acid $(0.2 \mathrm{mmol}, 29.6 \mathrm{mg})$ and sulfonamide $1 \mathrm{~b}(0.1$ $\mathrm{mmol}, 22.7 \mathrm{mg})$ were stirred in toluene $(1.0 \mathrm{~mL})$ at $35^{\circ} \mathrm{C}$ in a water bath. Then DIPEA $(0.48 \mathrm{mmol}, 79.3 \mu \mathrm{L}), \mathrm{PivCl}(0.24 \mathrm{mmol}, 29.4$ $\mu \mathrm{L}$ ) was added. The mixture was stirred at $35^{\circ} \mathrm{C}$ for $24 \mathrm{~h}$. Then the reaction mixture was subjected to column chromatography on silica gel and eluted with petroleum ether and acetone (v/v, 10:1) to afford $\mathbf{3 b}$. 


\section{Scale-up version of the asymmetric reaction}<smiles>CS(=O)(=O)c1ccccc1Br</smiles>

$1 \mathrm{~b}$<smiles>CC(C)OC(=O)OC(=O)/C=C/c1ccc([N+](=O)[O-])cc1</smiles>

21<smiles>CN(C(=O)/C=C/c1ccc([N+](=O)[O-])cc1)c1ccccc1Br</smiles>

$3 m$

$1.26 \mathrm{~g}$

$90 \%$ yield

$93 \%$ ee

Under an atmosphere of nitrogen, $(R)$-HBTM C3 (10 mol \%, $0.35 \mathrm{mmol}, 0.09 \mathrm{~g}), 3 \AA$ M.S. $(0.53 \mathrm{~g})$, sulfonamide $1 \mathrm{~b}$ (3.5 mmol, 0.79 g) and $\alpha, \beta$-unsaturated carbonic anhydride $2 \mathrm{I}(5.25 \mathrm{mmol}, 1.47 \mathrm{~g})$ were stirred in toluene $(35 \mathrm{~mL})$ at $35^{\circ} \mathrm{C}$ in a water bath. Then DIPEA $(7 \mathrm{mmol}, 1.16 \mathrm{~mL}$ ) was added. After stirring for $24 \mathrm{~h}$ (detection by TLC), the mixture was filtered through a plug celite, and the filtrate was concentrated under reduced pressure. Subsequently, the resulting residue was dissolved in DCM (50 mL) and washed with brine $\left(3 \times 50 \mathrm{~mL}\right.$ ). The organic solution was dried over $\mathrm{Na}_{2} \mathrm{SO}_{4}$ and concentrated.Then the reaction mixture was subjected to column chromatography on silica gel and eluted with petroleum ether and acetone ( $\mathrm{v} / \mathrm{v}, 10: 1)$ to afford $3 \mathrm{~m}$ as a light yellow solid (1.26 g). 


\section{The transformation of chiral product}

The general procedure for hydrogenation according to the literature procedure. ${ }^{3}$

To a solution of $3 \mathrm{~m}(1.0$ equiv, $0.1 \mathrm{mmol}, 40.2 \mathrm{mg})$ in ethyl acetate $(0.1 \mathrm{M}), \mathrm{Pd} / \mathrm{C}(10 \% \mathrm{w} / \mathrm{w})$ was added. Air was removed and the flask was filled with $\mathrm{H}_{2}$. The reaction mixture was stirred for $4 \mathrm{~h}$ under $\mathrm{H}_{2}$ atmosphere at $40{ }^{\circ} \mathrm{C}$ in a water bath (TLC monitoring), then it was cooled to room temperature and filtered over celite pad with dichloromethane. The volatiles were removed under reduced pressure. The residue was subjected to column chromatography on silica gel and eluted with hexanes/acetone (v/v, 5:1) to to afford $\mathbf{5}$<smiles>CN(C(=O)CCc1ccc([N+](=O)[O-])cc1)c1ccccc1C(C)(C)C</smiles>

5

White solid. m.p. $170-172{ }^{\circ} \mathrm{C} .39 .1 \mathrm{mg}, 97 \%$ yield, $93 \%$ ee. Specific rotation $[\alpha]^{20}{ }_{\mathrm{D}}=+47.8\left(c=0.36, \mathrm{CH}_{2} \mathrm{Cl}_{2}\right)$. Dissolved in $i-\mathrm{PrOH}$ for HPLC; HPLC (Chiral ID column, $i-\mathrm{PrOH} / n$-hexane $=20 / 80$, flow rate: $1.0 \mathrm{~mL} / \mathrm{min}, 254 \mathrm{~nm})$ : $\operatorname{tr}(\operatorname{major})=44.79 \mathrm{~min}, \operatorname{tr}(\operatorname{minor})=50.51$ $\min$.

${ }^{1} \mathrm{H}$ NMR $\left(400 \mathrm{MHz}, \mathrm{CDCl}_{3}\right) \delta=7.60(\mathrm{dd}, J=8.2,1.5,1 \mathrm{H}), 7.38(\mathrm{ddd}, J=8.3,7.2,1.5,1 \mathrm{H}), 7.18(\mathrm{td}, J=7.5,1.6,1 \mathrm{H}), 6.87-6.72(\mathrm{~m}$, $3 \mathrm{H}), 6.60-6.52(\mathrm{~m}, 2 \mathrm{H}), 3.46(\mathrm{~s}, 3 \mathrm{H}), 2.92-2.71(\mathrm{~m}, 2 \mathrm{H}), 2.35(\mathrm{t}, J=7.5,2 \mathrm{H}), 1.41(\mathrm{~s}, 9 \mathrm{H})$.

${ }^{13} \mathrm{C}\left\{{ }^{1} \mathrm{H}\right\}$ NMR $\left(101 \mathrm{MHz}, \mathrm{CDCl}_{3}\right) \delta 174.5,148.8,144.8,133.2,131.4,130.4,130.3,129.9,129.4,127.4,115.2,42.2,39.5,36.3,32.2$, 29.8.

HRMS (ESI+) $\mathrm{m} / \mathrm{z}$ calcd for $\mathrm{C}_{20} \mathrm{H}_{25} \mathrm{~N}_{2} \mathrm{O}_{5} \mathrm{~S}[\mathrm{M}+\mathrm{H}]^{+}: 405.1479$, found: 405.1486 .

IR (neat) 3372, 2959, 1699, 1626, 1518, 1487, 1438, 1351, 1285, 1146, 1068, 963, 826, 766, 542, 501.

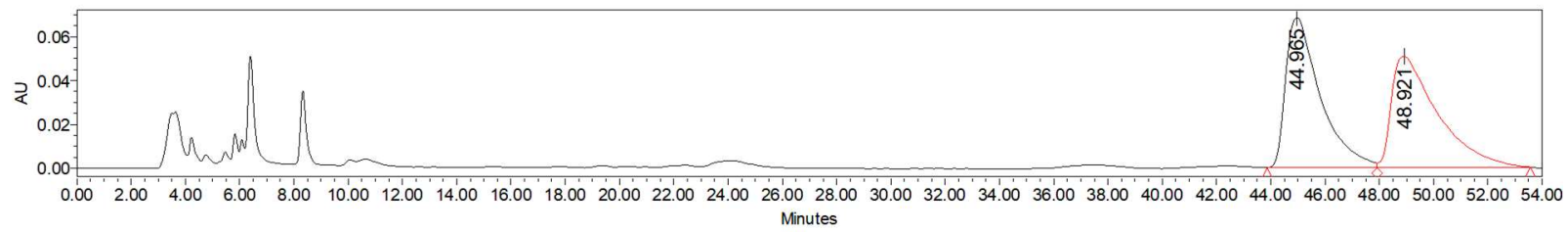

\begin{tabular}{|r|r|r|r|}
\hline & Retention Time & Area & \% Area \\
\hline 1 & 44.965 & 6057112 & 50.37 \\
\hline 2 & 48.921 & 5969141 & 49.63 \\
\hline
\end{tabular}

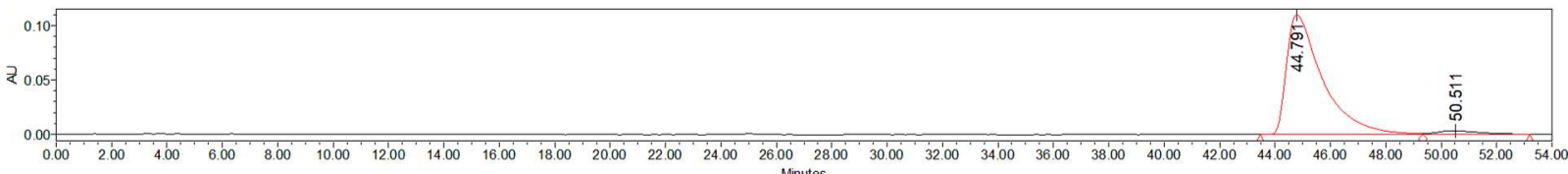

\begin{tabular}{|r|r|r|r|}
\hline & Retention Time & \multicolumn{1}{c|}{ Area } & \multicolumn{1}{c|}{$\%$ Area } \\
\hline 1 & 44.791 & 9910975 & 96.56 \\
\hline 2 & 50.511 & 353456 & 3.44 \\
\hline
\end{tabular}




\section{The side product analysis}

Unlike 1a, sulfonamide 1b provides a lower yield. Careful analysis of reaction mixture by TLC replied that a side product was generated, which was responsible for low isolated yield. The side product, arising from the nucleophilic attach of anilide anion to isopropoxycarbonyl unit, was assigned to compound $\mathbf{4 b}$ by ${ }^{1} \mathrm{H}$ NMR spectra. The polarities of the two products are so similar that it is easy to obtain a mixture of $\mathbf{3 b}$ and $\mathbf{4 b}$. The side product $\mathbf{4 b}$ is not collected in this reaction because the fluorescence is weak and the amount is small. And it is synthesized from benzoic acid-derived anhydride and sulfonamide $\mathbf{1 b}$.

\subsection{The determine of side product $\mathbf{4 b}$}

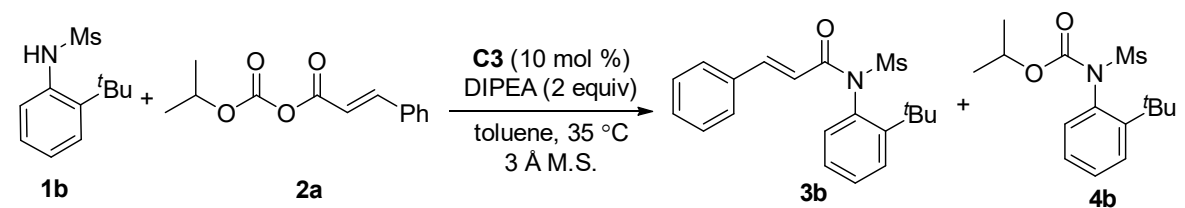

Spectrogram 1: A mixture of $\mathbf{3 b}$ and $\mathbf{4 b}$ in $\mathrm{CDCl}_{3}$

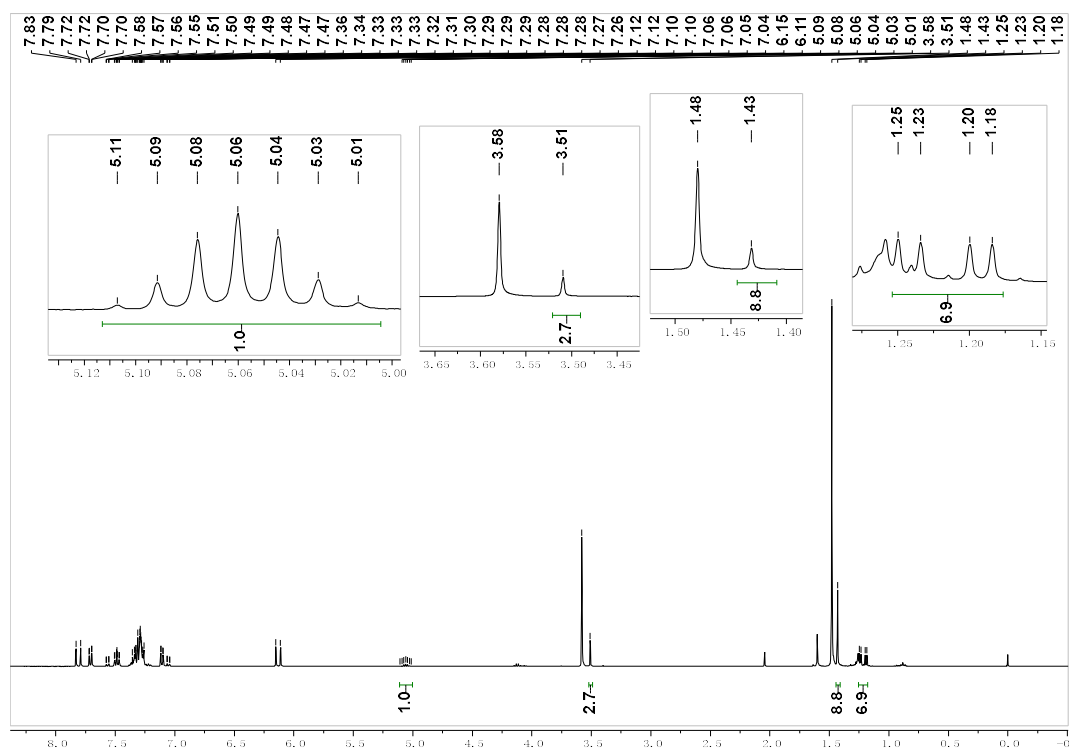

Spectrogram 2: $4 \mathrm{~b}$ in $\mathrm{CDCl}_{3}$

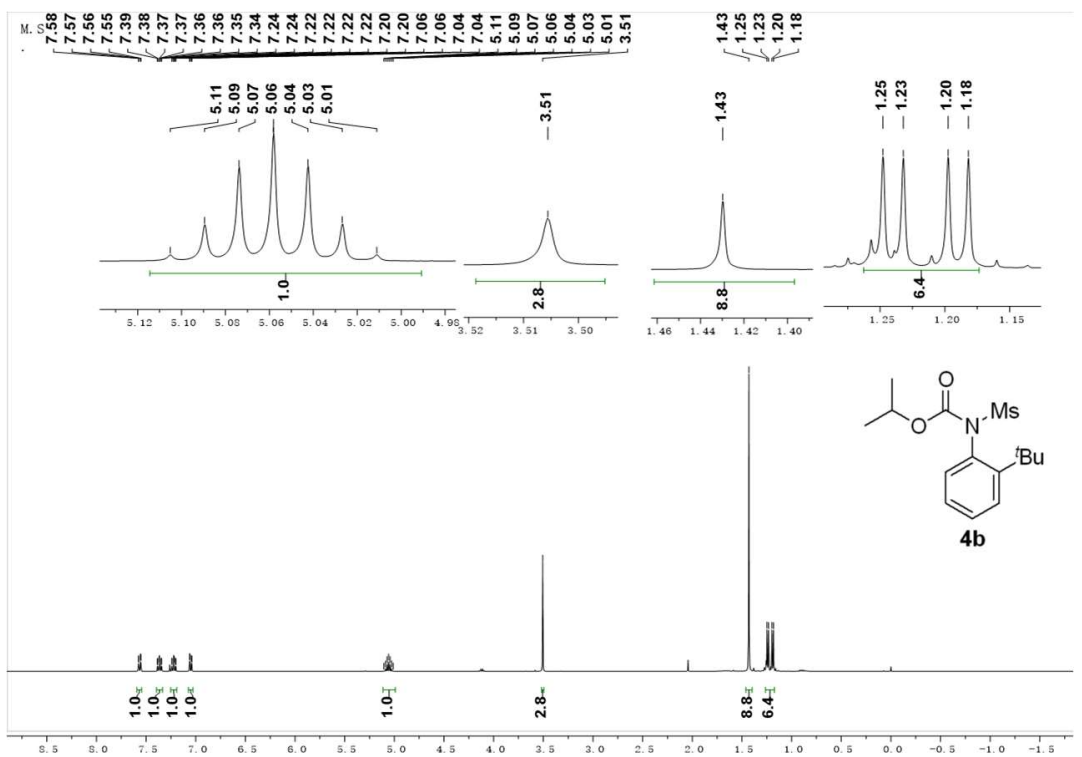




\subsection{The enantiomeric excess of side product $4 a$}

When the reaction was performed with $1 \mathrm{a}(0.1 \mathrm{mmol}), \mathbf{2 a}(3.0$ equiv), HBTM C3 $(10 \mathrm{~mol} \%)$ and $3 \AA$ M.S. (10 mg) in toluene (1 mL) at $35^{\circ} \mathrm{C}$ in a water bath, a mixture of $3 \mathbf{a}$ and $4 \mathbf{a}$ were obtained. The enantiomeric excess of side product $4 \mathbf{a}$ was determined by HPLC analysis on a chiral stationary phase.

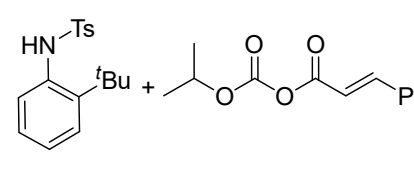

$1 \mathbf{a}$
$0.1 \mathrm{mmo}$
3.0 equiv

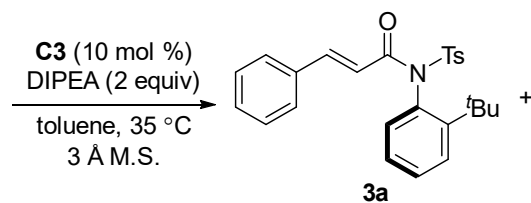

$90 \%$ yield, $89 \%$ ee

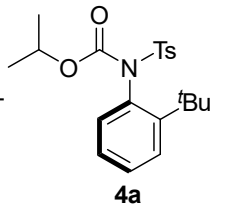

trace, $94 \%$ ee

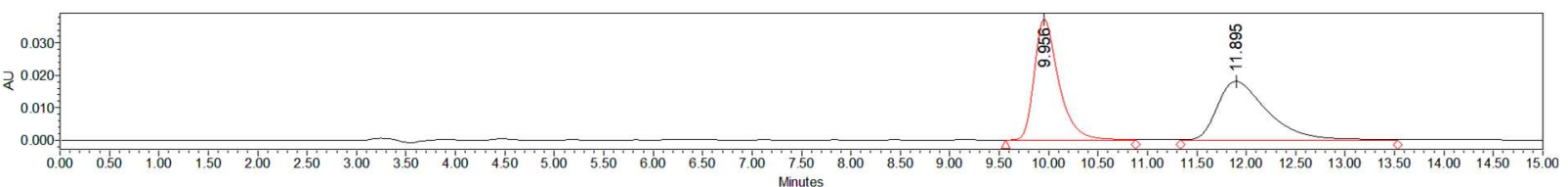

\begin{tabular}{|r|r|r|r|}
\hline & Retention Time & Area & \% Area \\
\hline 1 & 9.956 & 640951 & 49.99 \\
\hline 2 & 11.895 & 641217 & 50.01 \\
\hline
\end{tabular}

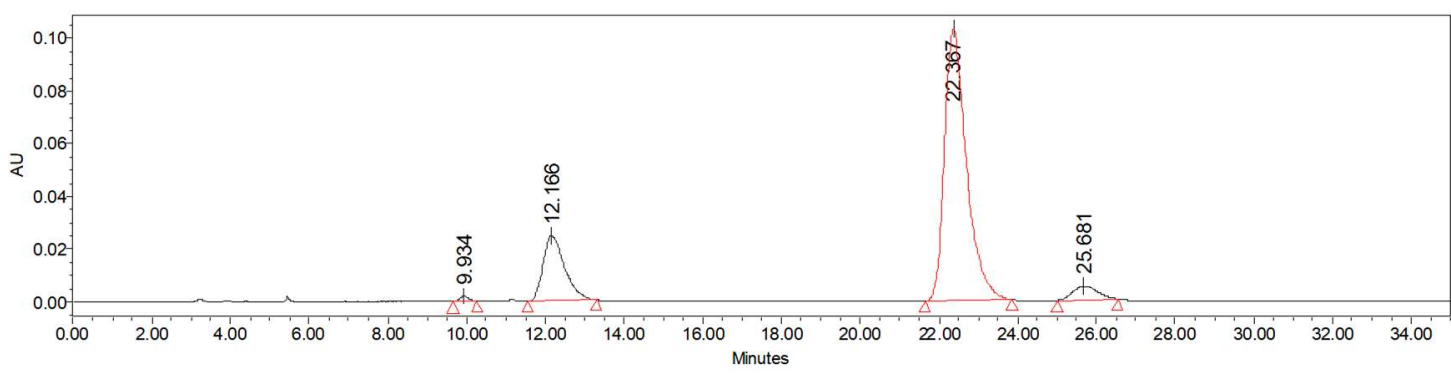

\begin{tabular}{|r|r|r|r|}
\hline & Retention Time & \multicolumn{1}{c|}{ Area } & \% Area \\
\hline 1 & 9.934 & 28080 & 0.54 \\
\hline 2 & 12.166 & 924562 & 17.93 \\
\hline 3 & 22.367 & 3982074 & 77.21 \\
\hline 4 & 25.681 & 222543 & 4.32 \\
\hline
\end{tabular}




\section{Characterization of the products}

$N$-(2-(Tert-butyl)phenyl)-N-tosylcinnamamide (3a)<smiles>Cc1ccc(S(=O)(=O)N(C(=O)/C=C/c2ccccc2)c2ccccc2Br)cc1</smiles>

$3 a$

White solid. m.p. $66-69^{\circ} \mathrm{C}$. $39.4 \mathrm{mg}, 91 \%$ yield, $87 \%$ ee. Specific rotation $[\alpha]^{20}{ }_{\mathrm{D}}=+116.4\left(c=0.42, \mathrm{CH}_{2} \mathrm{Cl}_{2}\right)$. Dissolved in $i-\mathrm{PrOH}$ for HPLC; HPLC (Chiral ID column, $i$-PrOH $/ n$-hexane = 15/85, flow rate: $1.0 \mathrm{~mL} / \mathrm{min}, 254 \mathrm{~nm}$ ): $\operatorname{tr}($ major $)=21.30 \mathrm{~min}, \operatorname{tr}(\mathrm{minor})=24.57$ $\min$.

${ }^{1} \mathrm{H}$ NMR $\left(400 \mathrm{MHz}, \mathrm{CDCl}_{3}\right) \delta=8.04(\mathrm{~d}, J=8.3,2 \mathrm{H}), 7.74(\mathrm{dd}, J=8.2,1.6,1 \mathrm{H}), 7.68(\mathrm{~d}, J=15.5,1 \mathrm{H}), 7.48$ (ddd, $J=8.4,7.2,1.5$, $1 \mathrm{H}), 7.37(\mathrm{~d}, J=8.1,2 \mathrm{H}), 7.32-7.20(\mathrm{~m}, 6 \mathrm{H}), 6.80(\mathrm{dd}, J=7.8,1.5,1 \mathrm{H}), 6.11(\mathrm{~d}, J=15.5,1 \mathrm{H}), 2.46(\mathrm{~s}, 3 \mathrm{H}), 1.56(\mathrm{~s}, 9 \mathrm{H})$.

${ }^{13} \mathrm{C}\left\{{ }^{1} \mathrm{H}\right\}$ NMR $\left(101 \mathrm{MHz}, \mathrm{CDCl}_{3}\right) \delta 165.7,150.2,145.5,145.0,136.2,134.3,134.2,131.3,130.7,130.6,130.2,130.1,129.3,128.8$, 128.2, 127.1, 118.1, 36.7, 32.6, 21.7.

HRMS (ESI+) $\mathrm{m} / \mathrm{z}$ calcd for $\mathrm{C}_{26} \mathrm{H}_{27} \mathrm{NNaO}_{3} \mathrm{~S}[\mathrm{M}+\mathrm{Na}]^{+}: 456.1604$, found: 456.1606 .

IR (neat) 2963, 2362, 1683, 1619, 1488, 1443, 1360, 1325, 1282, 1155, 1087, 984, 913, 856, 814, 762, 731, 666, 565, 490.
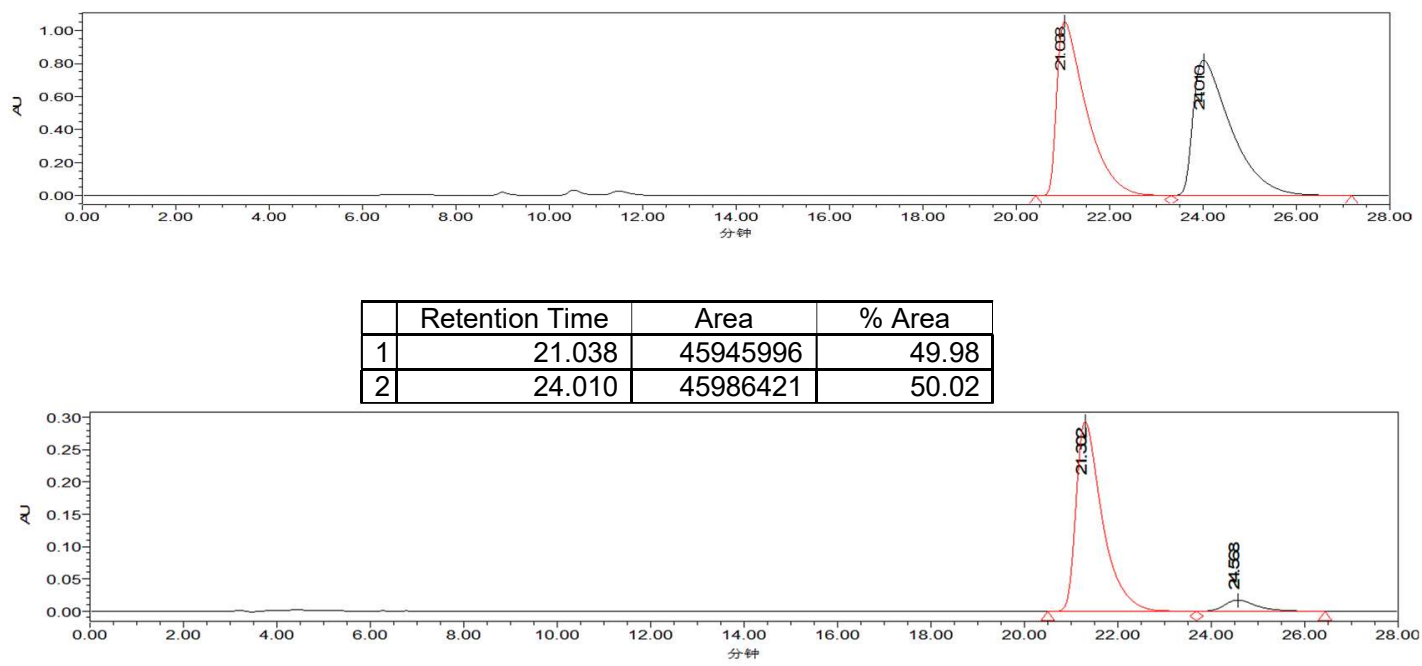

\begin{tabular}{|r|r|r|r|}
\hline & Retention Time & \multicolumn{1}{c|}{ Area } & \% Area \\
\hline 1 & 21.302 & 11475429 & 93.26 \\
\hline 2 & 24.568 & 829559 & 6.74 \\
\hline
\end{tabular}


$N$-(2-(Tert-butyl)phenyl)- $N$-(methylsulfonyl)cinnamamide (3b)<smiles>CN(C(=O)/C=C/c1ccccc1)c1ccccc1Br</smiles>

White solid. m.p. $39-45^{\circ} \mathrm{C} .31 .4 \mathrm{mg}, 88 \%$ yield, $95 \%$ ee. Specific rotation $[\alpha]^{20}{ }_{D}=+108.7\left(c=0.36, \mathrm{CH}_{2} \mathrm{Cl}_{2}\right)$. Dissolved in $i-\mathrm{PrOH}$ for HPLC; HPLC (Chiral ID column, $i-\mathrm{PrOH} / n$-hexane = 15/85, flow rate: $1.0 \mathrm{~mL} / \mathrm{min}, 254 \mathrm{~nm}$ ): $\operatorname{tr}($ major $)=23.91 \mathrm{~min}, \operatorname{tr}(\operatorname{minor})=28.39$ min.

${ }^{1} \mathrm{H}$ NMR $\left(400 \mathrm{MHz}, \mathrm{CDCl}_{3}\right) \delta=7.81(\mathrm{~d}, J=15.5,1 \mathrm{H}), 7.71(\mathrm{dd}, J=8.2,1.5,1 \mathrm{H}), 7.49(\mathrm{ddd}, J=8.4,7.3,1.5,1 \mathrm{H}), 7.39-7.26(\mathrm{~m}$, $6 \mathrm{H}), 7.11(\mathrm{dd}, J=7.8,1.5,1 \mathrm{H}), 6.13(\mathrm{~d}, J=15.5,1 \mathrm{H}), 3.58(\mathrm{~s}, 3 \mathrm{H}), 1.48(\mathrm{~s}, 9 \mathrm{H})$.

${ }^{13} \mathrm{C}\left\{{ }^{1} \mathrm{H}\right\}$ NMR $\left(101 \mathrm{MHz}, \mathrm{CDCl}_{3}\right) \delta 167.2,149.4,146.2,134.0,133.2,131.7,130.8,130.5,130.4,128.9,128.3,127.6,117.7,42.4$, 36.4, 32.3.

HRMS (ESI+) $\mathrm{m} / \mathrm{z}$ calcd for $\mathrm{C}_{20} \mathrm{H}_{23} \mathrm{NNaO}_{3} \mathrm{~S}[\mathrm{M}+\mathrm{Na}]^{+}: 308.1291$, found: 308.1296 .

IR (neat) 2965, 1679, 1619, 1488, 1443, 1353, 1153, 965, 856, 766, 707, 545.

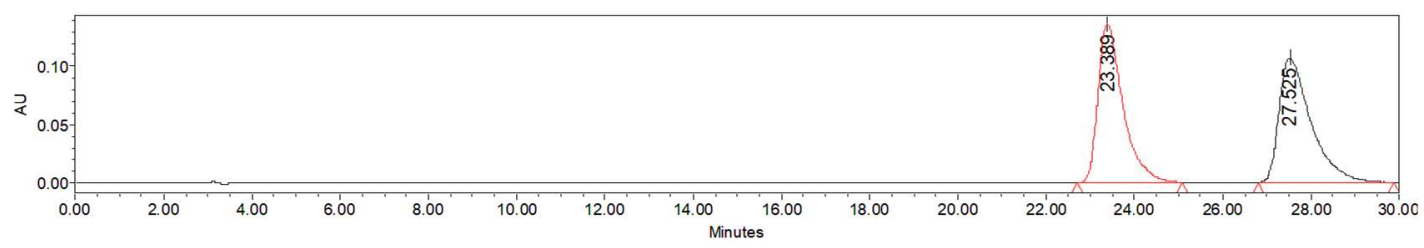

\begin{tabular}{|l|r|r|r|}
\hline & Retention Time & Area & \% Area \\
\hline 1 & 23.389 & 5341502 & 50.03 \\
\hline 2 & 27.525 & 5334727 & 49.97 \\
\hline
\end{tabular}

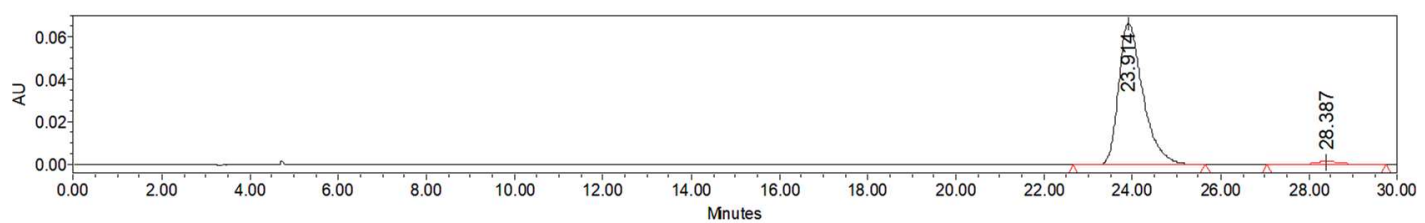

\begin{tabular}{|r|r|r|r|}
\hline & Retention Time & Area & \% Area \\
\hline 1 & 23.914 & 2524927 & 97.29 \\
\hline 2 & 28.387 & 70342 & 2.71 \\
\hline
\end{tabular}


(E)-N-(2-(Tert-butyl)phenyl)-N-(methylsulfonyl)-3-(p-tolyl)acrylamide (3c):<smiles>Cc1ccc(/C=C/C(=O)N(C)c2ccccc2Br)cc1</smiles>

White solid. m.p. $160-170{ }^{\circ} \mathrm{C}$. $28.1 \mathrm{mg}, 76 \%$ yield, $97 \%$ ee. Specific rotation $[\alpha]^{20}{ }_{\mathrm{D}}=+113.9\left(c=0.58, \mathrm{CH}_{2} \mathrm{Cl}_{2}\right)$. Dissolved in $i-$ $\mathrm{PrOH}$ for HPLC; HPLC (Chiral IC column, $i-\mathrm{PrOH} / n$-hexane $=10 / 90$, flow rate: $1.0 \mathrm{~mL} / \mathrm{min}, 254 \mathrm{~nm})$ : $\operatorname{tr}(\operatorname{minor})=9.09 \mathrm{~min}$, $\operatorname{tr}(\mathrm{major})=$ $11.74 \mathrm{~min}$.

${ }^{1} \mathrm{H}$ NMR $\left(400 \mathrm{MHz}, \mathrm{CDCl}_{3}\right) \delta=7.79(\mathrm{~d}, J=15.5,1 \mathrm{H}), 7.70(\mathrm{dd}, J=8.2,1.5,1 \mathrm{H}), 7.48(\mathrm{ddd}, J=8.3,7.2,1.5,1 \mathrm{H}), 7.29(\mathrm{td}, J=7.5$, $1.5,1 \mathrm{H}), 7.18(\mathrm{~d}, J=8.2,2 \mathrm{H}), 7.14-7.08(\mathrm{~m}, 3 \mathrm{H}), 6.08(\mathrm{~d}, J=15.5,1 \mathrm{H}), 3.57(\mathrm{~s}, 3 \mathrm{H}), 2.33(\mathrm{~s}, 3 \mathrm{H}), 1.48(\mathrm{~s}, 9 \mathrm{H})$.

${ }^{13} \mathrm{C}\left\{{ }^{1} \mathrm{H}\right\}$ NMR $\left(101 \mathrm{MHz}, \mathrm{CDCl}_{3}\right) \delta 167.3,149.4,146.3,141.5,133.2,131.7,131.3,130.4,130.4,129.6,128.3,127.6,116.6,42.4$, 36.4, 32.3, 21.5.

HRMS (ESI+) $\mathrm{m} / \mathrm{z}$ calcd for $\mathrm{C}_{21} \mathrm{H}_{25} \mathrm{NaO}_{3} \mathrm{~S}[\mathrm{M}+\mathrm{Na}]^{+}: 394.1447$, found: 394.1444 .

IR (neat) 2963, 2362, 1678, 1611, 1487, 1352, 1323, 1280, 1151, 965, 916, 812, 763, 544, 498.

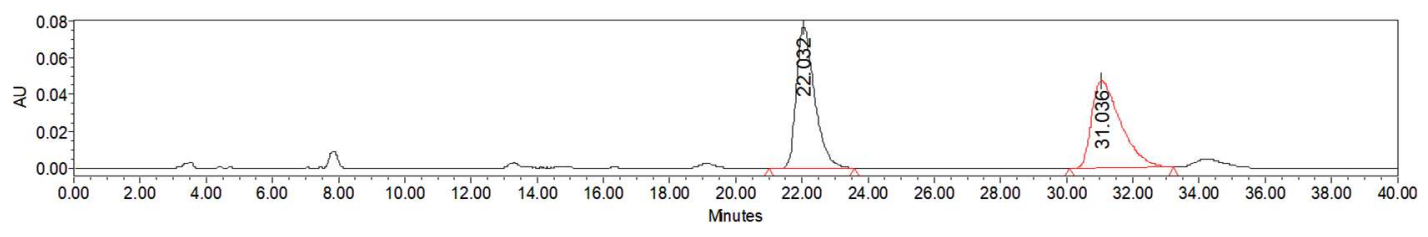

\begin{tabular}{|r|r|r|r|}
\hline & Retention Time & Area & \% Area \\
\hline 1 & 22.032 & 2944466 & 50.36 \\
\hline 2 & 31.036 & 2902030 & 49.64 \\
\hline
\end{tabular}

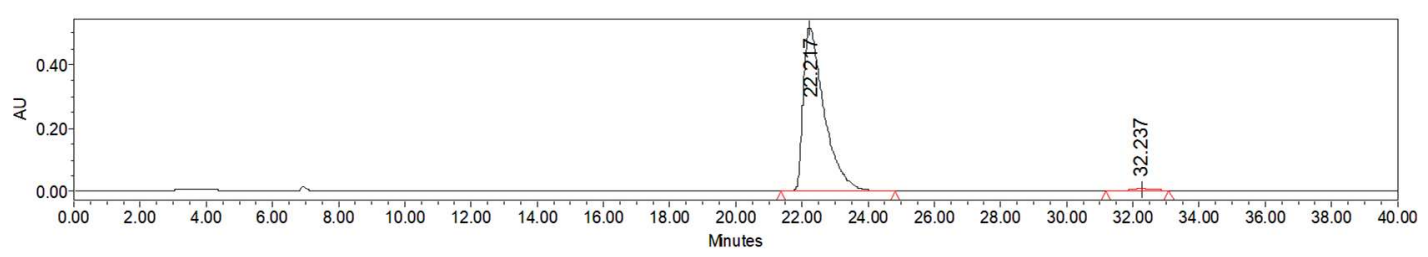

\begin{tabular}{|r|r|r|r|}
\hline & Retention Time & \multicolumn{1}{c|}{ Area } & \% Area \\
\hline 1 & 22.217 & 22957019 & 98.26 \\
\hline 2 & 32.237 & 407480 & 1.74 \\
\hline
\end{tabular}


(E)-N-(2-(Tert-butyl)phenyl)-3-(4-methoxyphenyl)- $N$-(methylsulfonyl)acrylamide (3d):<smiles>COc1ccc(/C=C/C(=O)N(c2ccccc2C(C)(C)C)S(C)(=O)=O)cc1</smiles>

3d

White solid. m.p. $54-58{ }^{\circ} \mathrm{C}$. $24.2 \mathrm{mg}, 63 \%$ yield, $95 \%$ ee. Specific rotation $[\alpha]^{20}{ }_{D}=+130.1\left(c=0.30, \mathrm{CH}_{2} \mathrm{Cl}_{2}\right)$. Dissolved in $i-\mathrm{PrOH}$ for HPLC; HPLC (Chiral ID column, $i$-PrOH $/ n$-hexane = 20/80, flow rate: $1.0 \mathrm{~mL} / \mathrm{min}, 254 \mathrm{~nm}$ ): $\operatorname{tr}$ (major) $=33.64 \mathrm{~min}, \operatorname{tr}(\operatorname{minor})=47.79$ min.

${ }^{1} \mathrm{H}$ NMR $\left(400 \mathrm{MHz}, \mathrm{CDCl}_{3}\right) \delta=7.76(\mathrm{~d}, J=15.4,1 \mathrm{H}), 7.70(\mathrm{dd}, J=8.2,1.6,1 \mathrm{H}), 7.47$ (ddd, $\left.J=8.3,7.2,1.5,1 \mathrm{H}\right), 7.32-7.20(\mathrm{~m}$, $3 \mathrm{H}), 7.10(\mathrm{dd}, J=7.8,1.6,1 \mathrm{H}), 6.85-6.79(\mathrm{~m}, 2 \mathrm{H}), 5.98(\mathrm{~d}, J=15.5,1 \mathrm{H}), 3.79(\mathrm{~s}, 3 \mathrm{H}), 3.57(\mathrm{~s}, 3 \mathrm{H}), 1.47(\mathrm{~s}, 9 \mathrm{H})$.

${ }^{13} \mathrm{C}\left\{{ }^{1} \mathrm{H}\right\}$ NMR $\left(101 \mathrm{MHz}, \mathrm{CDCl}_{3}\right) \delta 167.5,161.8,149.4,146.0,133.3,131.8,130.3,130.3,130.1,127.5,126.7,115.1,114.4,55.4$, 42.4, 36.4, 32.3.

HRMS (ESI+) $\mathrm{m} / \mathrm{z}$ calcd for $\mathrm{C}_{21} \mathrm{H}_{25} \mathrm{NNaO}_{4} \mathrm{~S}[\mathrm{M}+\mathrm{Na}]^{+}:$: 410.1397, found: 410.1403 .

IR (neat) 3730, 2963, 2362, 2336, 1676, 1598, 1512, 1485, 1350, 1260, 1149, 1028, 964, 828, 758, 542.

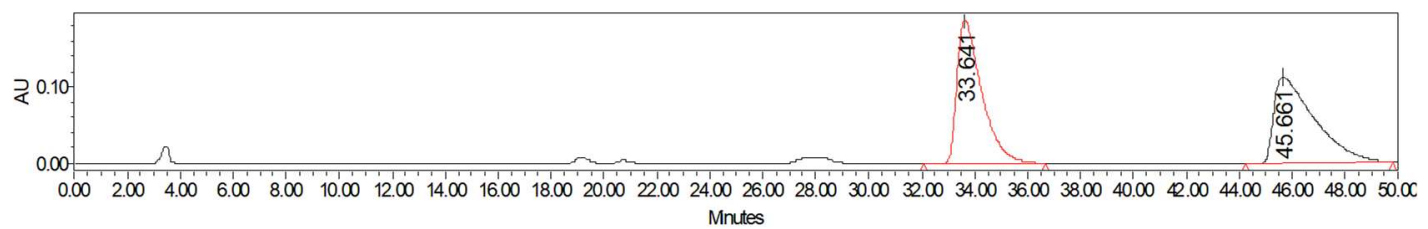

\begin{tabular}{|r|r|r|r|}
\hline & Retention Time & Area & \% Area \\
\hline 1 & 33.641 & 12099533 & 50.22 \\
\hline 2 & 45.661 & 11995884 & 49.78 \\
\hline
\end{tabular}

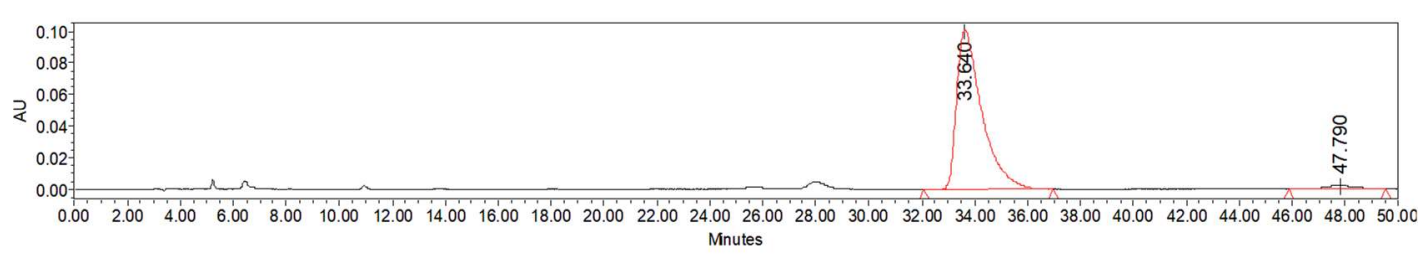

\begin{tabular}{|r|r|r|r|}
\hline & Retention Time & Area & \% Area \\
\hline 1 & 33.640 & 6678123 & 97.49 \\
\hline 2 & 47.790 & 171728 & 2.51 \\
\hline
\end{tabular}


(E)-3-(Benzod $[1,3]$ dioxol-5-yl)- $N$-(2-(tert-butyl)phenyl)- $N$-(methylsulfonyl)acrylamide (3e)<smiles>CC(C)(C)N(C(=O)/C=C/c1ccc2c(c1)OCO2)c1ccccc1S(C)(=O)=O</smiles>

$3 e$

White solid. m.p. $144-149{ }^{\circ} \mathrm{C}$. $36.2 \mathrm{mg}, 90 \%$ yield, $94 \%$ ee. Specific rotation $[\alpha]^{20}{ }_{D}=+96.4\left(c=0.45, \mathrm{CH}_{2} \mathrm{Cl}_{2}\right)$. Dissolved in $i-\mathrm{PrOH}$ for HPLC; HPLC (Chiral ID column, $i-\mathrm{PrOH} / n$-hexane $=20 / 80$, flow rate: $1.0 \mathrm{~mL} / \mathrm{min}, 254 \mathrm{~nm}$ ): $\operatorname{tr}(\operatorname{minor})=32.35 \mathrm{~min}, \operatorname{tr}(\mathrm{major})=36.67$ $\min$.

${ }^{1} \mathrm{H}$ NMR $\left(400 \mathrm{MHz}, \mathrm{CDCl}_{3}\right) \delta=7.75-7.67(\mathrm{~m}, 2 \mathrm{H}), 7.51-7.44(\mathrm{~m}, 1 \mathrm{H}), 7.33-7.26(\mathrm{~m}, 1 \mathrm{H}), 7.10(\mathrm{dd}, J=7.8,1.5,1 \mathrm{H}), 6.87(\mathrm{dd}, \mathrm{J}$ $=8.1,1.6,1 \mathrm{H}), 6.76(\mathrm{~d}, J=8.0,1 \mathrm{H}), 6.67(\mathrm{~d}, J=1.7,1 \mathrm{H}), 5.97(\mathrm{~s}, 2 \mathrm{H}), 5.93(\mathrm{~d}, J=15.4,1 \mathrm{H}), 3.57(\mathrm{~s}, 3 \mathrm{H}), 1.47(\mathrm{~s}, 9 \mathrm{H})$.

${ }^{13} \mathrm{C}\left\{{ }^{1} \mathrm{H}\right\}$ NMR $\left(101 \mathrm{MHz}, \mathrm{CDCl}_{3}\right) \delta 167.3,150.1,149.4,148.3,146.0,133.2,131.8,130.5,130.4,128.5,127.6,125.4,115.6,108.6$, 106.3, 101.6, 42.4, 36.4, 32.3.

HRMS (ESI+) $\mathrm{m} / \mathrm{z}$ calcd for $\mathrm{C}_{21} \mathrm{H}_{23} \mathrm{~N}_{2} \mathrm{NaO}_{5} \mathrm{~S}[\mathrm{M}+\mathrm{Na}]^{+}: 4241189$, found: 424.1183 .

IR (neat) 3729, 2963, 2362, 2336, 1676, 1598, 1490, 1447, 1351, 1250, 1151, 1100, 1036, 964, 927, 810, 758, 675, 542.

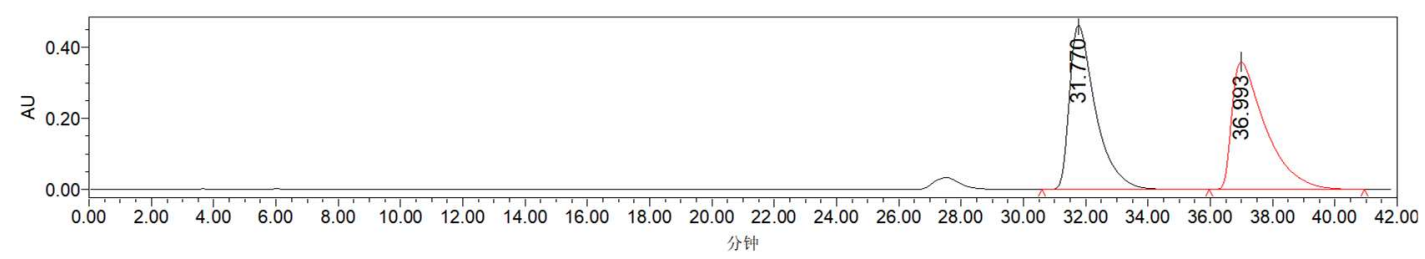

\begin{tabular}{|r|r|r|r|}
\hline & Retention Time & Area & \% Area \\
\hline 1 & 31.770 & 26812415 & 50.01 \\
\hline 2 & 36.993 & 26796589 & 49.99 \\
\hline
\end{tabular}

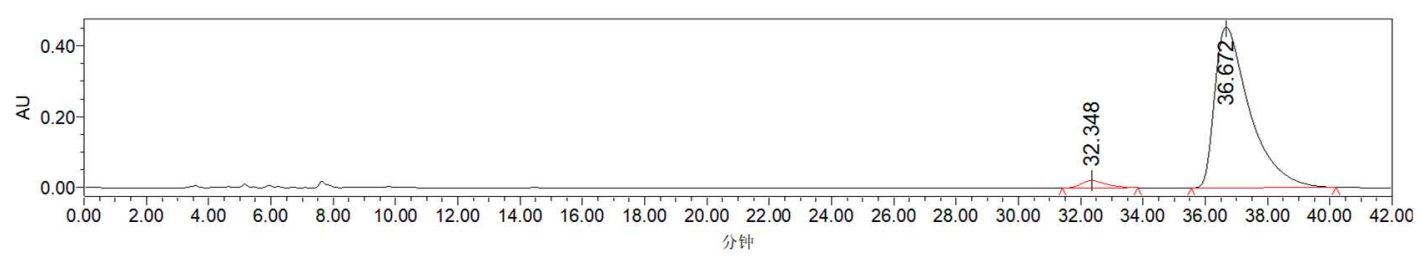

\begin{tabular}{|r|r|r|r|}
\hline & Retention Time & \multicolumn{1}{c|}{ Area } & \% Area \\
\hline 1 & 32.348 & 1139784 & 3.12 \\
\hline 2 & 36.672 & 35372927 & 96.88 \\
\hline
\end{tabular}


(E)-N-(2-(Tert-butyl)phenyl)-3-(4-fluorophenyl)- $N$-(methylsulfonyl)acrylamide (3f)<smiles>CN(C(=O)/C=C/c1ccc(F)cc1)c1ccccc1C(C)(C)C</smiles>

White solid. m.p. $55-59{ }^{\circ} \mathrm{C}$. $28.2 \mathrm{mg}, 78 \%$ yield, $96 \%$ ee. Specific rotation $[\alpha]^{20}{ }_{\mathrm{D}}=+136.0\left(c=0.24, \mathrm{CH}_{2} \mathrm{Cl}_{2}\right)$. Dissolved in $i-\mathrm{PrOH}$ for HPLC; HPLC (Chiral ID column, $i-\mathrm{PrOH} / n$-hexane = 20/80, flow rate: $1.0 \mathrm{~mL} / \mathrm{min}, 254 \mathrm{~nm}$ ): $\operatorname{tr}$ (major) $=18.12 \mathrm{~min}, \operatorname{tr}(\operatorname{minor})=21.75$ $\min$.

${ }^{1} \mathrm{H}$ NMR $\left(400 \mathrm{MHz}, \mathrm{CDCl}_{3}\right) \delta=7.77(\mathrm{~d}, J=15.5,1 \mathrm{H}), 7.71(\mathrm{~d}, J=8.1,1 \mathrm{H}), 7.49(\mathrm{t}, J=7.7,1 \mathrm{H}), 7.34-7.25(\mathrm{~m}, 3 \mathrm{H}), 7.10(\mathrm{dd}, J=$ $7.8,1.4,1 \mathrm{H}), 7.00(\mathrm{t}, J=8.4,2 \mathrm{H}), 6.05(\mathrm{~d}, J=15.5,1 \mathrm{H}), 3.58(\mathrm{~s}, 3 \mathrm{H}), 1.47(\mathrm{~s}, 9 \mathrm{H})$.

${ }^{13} \mathrm{C}\left\{{ }^{1} \mathrm{H}\right\}$ NMR $\left(101 \mathrm{MHz}, \mathrm{CDCl}_{3}\right) \delta 167.02,164.1(\mathrm{~d}, \mathrm{~J}=253.5 \mathrm{~Hz}), 149.4,144.9,133.1,131.8,130.5,130.4,130.3(\mathrm{~d}, \mathrm{~J}=9.0 \mathrm{~Hz})$, $130.2(\mathrm{~d}, J=4.0 \mathrm{~Hz}$ ), 127.6, 117.4, 116.1 (d, $J=22.2 \mathrm{~Hz}$ ), 42.4, 36.4, 32.3.

${ }^{19} \mathrm{~F}\left\{{ }^{1} \mathrm{H}\right\}$ NMR $\left(376 \mathrm{MHz}, \mathrm{CDCl}_{3}\right) \delta-108.2$.

HRMS (ESI+) $\mathrm{m} / \mathrm{z}$ calcd for $\mathrm{C}_{20} \mathrm{H}_{22} \mathrm{FNNaO}_{3} \mathrm{~S}[\mathrm{M}+\mathrm{Na}]^{+}: 398.1197$, found: 398.1205 .

IR (neat) 2965, 2362, 1679, 1622, 1599, 1509, 1416, 1353, 1278, 1232, 1153, 965, 831, 760, 544.

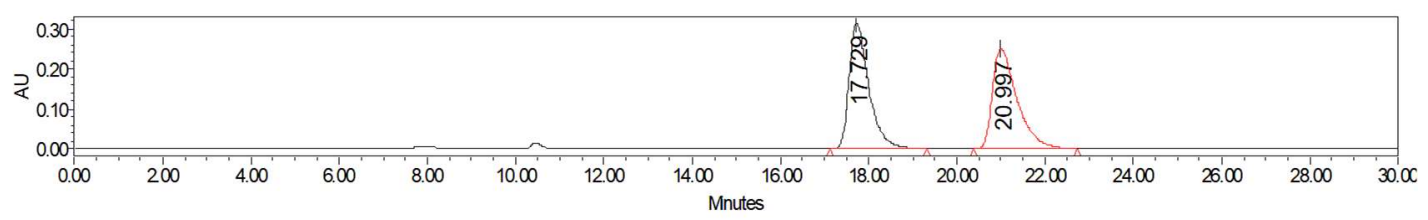

\begin{tabular}{|r|r|r|r|}
\hline & Retention Time & Area & \% Area \\
\hline 1 & 17.729 & 9724724 & 50.15 \\
\hline 2 & 20.997 & 9667631 & 49.85 \\
\hline
\end{tabular}

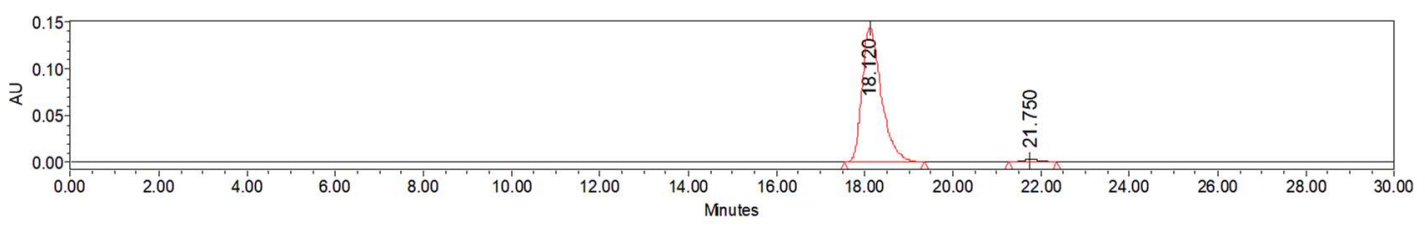

\begin{tabular}{|r|r|r|r|}
\hline & Retention Time & \multicolumn{1}{c|}{ Area } & \% Area \\
\hline 1 & 18.120 & 4403134 & 97.70 \\
\hline 2 & 21.750 & 103441 & 2.30 \\
\hline
\end{tabular}


<smiles>CN(C(=O)/C=C/c1ccc(Cl)cc1)c1ccccc1Br</smiles>

White solid. m.p. $127-129{ }^{\circ} \mathrm{C}$. $35.2 \mathrm{mg}, 90 \%$ yield, $94 \%$ ee. Specific rotation $[\alpha]^{20}{ }_{\mathrm{D}}=+114.4\left(c=0.42, \mathrm{CH}_{2} \mathrm{Cl}_{2}\right)$. Dissolved in $i-$ $\mathrm{PrOH}$ for HPLC; HPLC (Chiral ID column, $i-\mathrm{PrOH} / n$-hexane $=20 / 80$, flow rate: $1.0 \mathrm{~mL} / \mathrm{min}, 254 \mathrm{~nm}$ ): $\operatorname{tr}(\operatorname{major})=18.96 \mathrm{~min}$, tr (minor) $=24.45 \mathrm{~min}$.

${ }^{1} \mathrm{H}$ NMR $\left(400 \mathrm{MHz}, \mathrm{CDCl}_{3}\right) \delta=7.79-7.67(\mathrm{~m}, 2 \mathrm{H}), 7.48(\mathrm{ddd}, J=8.4,7.2,1.5,1 \mathrm{H}), 7.35-7.26(\mathrm{~m}, 3 \mathrm{H}), 7.19(\mathrm{~d}, J=8.5,2 \mathrm{H}), 7.09$ (dd, $J=7.8,1.5,1 \mathrm{H}), 6.08(\mathrm{~d}, J=15.5,1 \mathrm{H}), 3.56(\mathrm{~s}, 3 \mathrm{H}), 1.46(\mathrm{~s}, 9 \mathrm{H})$.

${ }^{13} \mathrm{C}\left\{{ }^{1} \mathrm{H}\right\}$ NMR $\left(101 \mathrm{MHz}, \mathrm{CDCl}_{3}\right) \delta 166.9,149.4,144.7,136.8,133.1,132.5,131.7,130.5,130.4,129.4,129.2,127.6,118.1,42.4$, 36.4, 32.3.

HRMS (ESI+) $\mathrm{m} / \mathrm{z}$ calcd for $\mathrm{C}_{20} \mathrm{H}_{22} \mathrm{CINNaO}_{3} \mathrm{~S}[\mathrm{M}+\mathrm{Na}]^{+}: 414.0901$ and 416.0872 , found: 414.0897 and 416.0866 .

IR (neat) 2964, 2362, 1679, 1619, 1489, 1406, 1353, 1321, 1275, 1153, 1092, 965, 821, 762, 544.

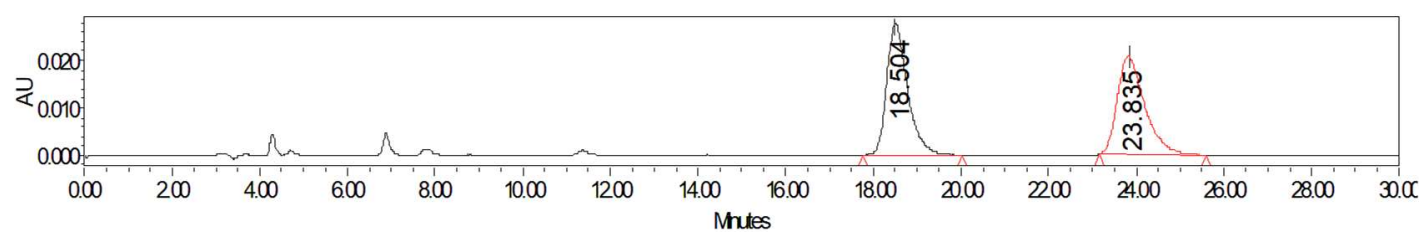

\begin{tabular}{|r|r|r|r|}
\hline & Retention Time & Area & \% Area \\
\hline 1 & 18.504 & 908969 & 50.36 \\
\hline 2 & 23.835 & 895882 & 49.64 \\
\hline
\end{tabular}

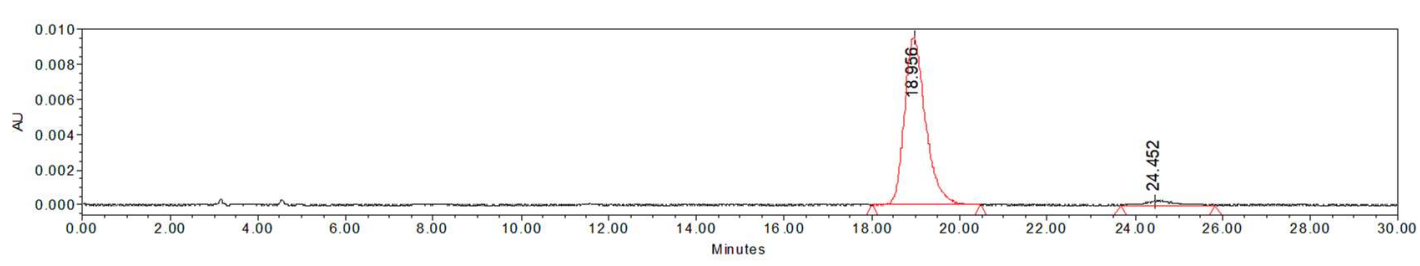

\begin{tabular}{|r|r|r|r|}
\hline & Retention Time & Area & \% Area \\
\hline 1 & 18.956 & 317666 & 96.63 \\
\hline 2 & 24.452 & 11066 & 3.37 \\
\hline
\end{tabular}


(E)-3-(4-Bromophenyl)-N-(2-(tert-butyl)phenyl)- $N$-(methylsulfonyl)acrylamide (3h):<smiles>CN(C(=O)/C=C/c1ccc(Br)cc1)c1ccccc1Br</smiles>

$3 h$

White solid. m.p. $105-109^{\circ} \mathrm{C} .41 .4 \mathrm{mg}, 95 \%$ yield, $94 \%$ ee. Specific rotation $[\alpha]^{20}{ }_{D}=+96.6\left(c=0.59, \mathrm{CH}_{2} \mathrm{Cl}_{2}\right)$. Dissolved in $i-\mathrm{PrOH}$ for HPLC; HPLC (Chiral ID column, $i-\mathrm{PrOH} / n$-hexane $=20 / 80$, flow rate: $1.0 \mathrm{~mL} / \mathrm{min}, 254 \mathrm{~nm}$ ): $\operatorname{tr}$ (major) $=19.91 \mathrm{~min}, \operatorname{tr}(\operatorname{minor})=26.72$ $\min$.

${ }^{1} \mathrm{H}$ NMR $\left(400 \mathrm{MHz}, \mathrm{CDCl}_{3}\right) \delta=7.78-7.68(\mathrm{~m}, 2 \mathrm{H}), 7.52-7.42(\mathrm{~m}, 3 \mathrm{H}), 7.30(\mathrm{td}, J=7.5,1.5,1 \mathrm{H}), 7.17-7.05(\mathrm{~m}, 3 \mathrm{H}), 6.11(\mathrm{~d}, J=$ 15.6, 1H), $3.57(\mathrm{~s}, 3 \mathrm{H}), 1.47(\mathrm{~s}, 9 \mathrm{H})$.

${ }^{13} \mathrm{C}\left\{{ }^{1} \mathrm{H}\right\}$ NMR $\left(101 \mathrm{MHz}, \mathrm{CDCl}_{3}\right) \delta 166.9,149.4,144.8,133.1,132.9,132.2,131.7,130.6,130.4,129.6,127.6,125.2,118.3,42.4$, 36.4, 32.3.

HRMS (ESI+) $\mathrm{m} / \mathrm{z}$ calcd for $\mathrm{C}_{20} \mathrm{H}_{22} \mathrm{BrNNaO}_{3} \mathrm{~S}$, [M+Na] $]^{+}: 458.0396$ and 460.0376 , found: 458.0389 and 460.0370 .

IR (neat) 2963, 2362, 1679, 1619, 1587, 1487, 1402, 1352, 1321, 1273, 1153, 1072, 1072, 965, 915, 817, 761, $544,498$.

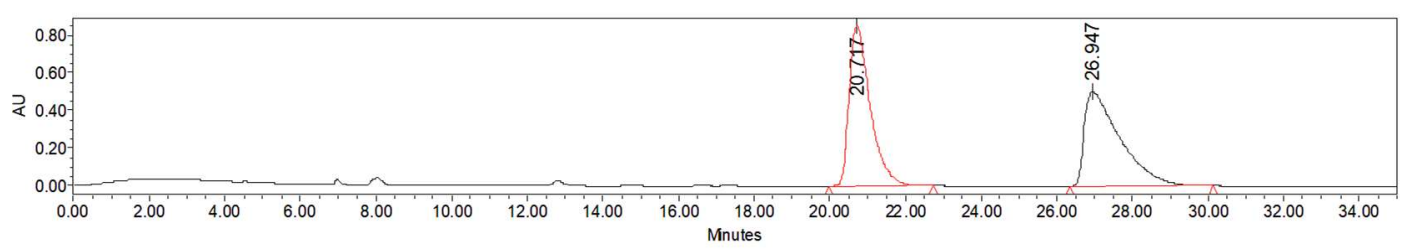

\begin{tabular}{|r|r|r|r|}
\hline & Retention Time & Area & \% Area \\
\hline 1 & 20.717 & 33069513 & 49.94 \\
\hline 2 & 26.947 & 33144122 & 50.06 \\
\hline
\end{tabular}

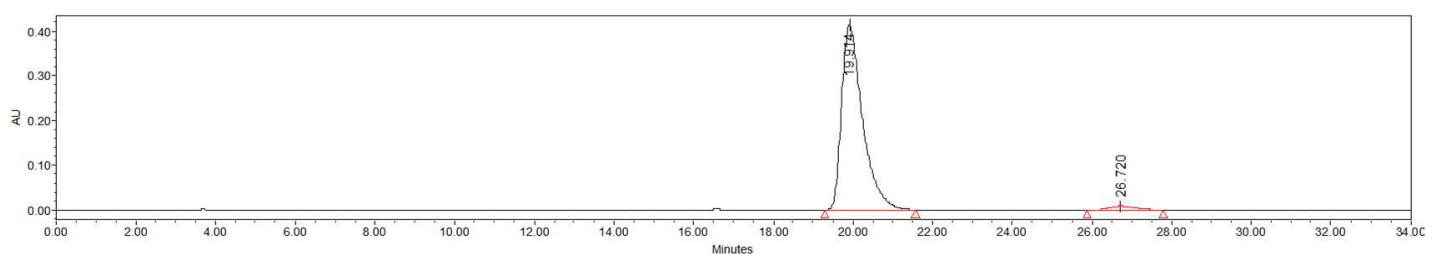

\begin{tabular}{|r|r|r|r|}
\hline & Retention Time & Area & \multicolumn{1}{c|}{$\%$ Area } \\
\hline 1 & 19.914 & 15083879 & 97.00 \\
\hline 2 & 26.720 & 466844 & 3.00 \\
\hline
\end{tabular}


(E)-3-(3-Bromophenyl)-N-(2-(tert-butyl)phenyl)- $N$-(methylsulfonyl)acrylamide (3i):<smiles>CN(C(=O)/C=C/c1cccc(Br)c1)c1ccccc1Br</smiles>

$3 \mathbf{i}$

White solid. m.p. $55-57{ }^{\circ} \mathrm{C}$. $32.6 \mathrm{mg}, 75 \%$ yield, $94 \%$ ee. Specific rotation $[\alpha]^{20}{ }_{D}=+102.1\left(c=0.68, \mathrm{CH}_{2} \mathrm{Cl}_{2}\right)$. Dissolved in $i-\mathrm{PrOH}$ for SFC; SFC (Chiralcel C1, $\mathrm{CO}_{2} / i-\mathrm{PrOH}=80 / 20$, flow rate $=1.0 \mathrm{~mL} / \mathrm{min}, 254 \mathrm{~nm}$ ): $\operatorname{tr}($ major $)=8.95 \mathrm{~min}, \operatorname{tr}($ minor $)=9.43 \mathrm{~min}$.

${ }^{1} \mathrm{H}$ NMR $\left(400 \mathrm{MHz}, \mathrm{CDCl}_{3}\right) \delta=7.72(\mathrm{ddd}, J=12.0,3.9,2.1,2 \mathrm{H}), 7.54-7.43(\mathrm{~m}, 2 \mathrm{H}), 7.43-7.38(\mathrm{~m}, 1 \mathrm{H}), 7.31(\mathrm{tt}, J=7.3,1.8,1 \mathrm{H})$, $7.19(\mathrm{dt}, J=3.5,1.6,2 \mathrm{H}), 7.10(\mathrm{dt}, J=7.8,1.8,1 \mathrm{H}), 6.11(\mathrm{dd}, J=15.6,2.2,1 \mathrm{H}), 3.57(\mathrm{~s}, 3 \mathrm{H}), 1.47(\mathrm{~s}, 9 \mathrm{H})$.

${ }^{13} \mathrm{C}\left\{{ }^{1} \mathrm{H}\right\}$ NMR $\left(101 \mathrm{MHz}, \mathrm{CDCl}_{3}\right) \delta 166.7,149.4,144.5,136.1,133.5,133.0,131.7,131.1,130.6,130.5,130.4,127.7,126.6,123.0$, $119.0,42.3,36.4,32.3$.

HRMS (ESI+) $\mathrm{m} / \mathrm{z}$ calcd for $\mathrm{C}_{20} \mathrm{H}_{22} \mathrm{BrNNaO}_{3} \mathrm{~S}$, [M+Na] $]^{+}: 458.0396$ and 460.0376 , found: 458.0387 and 460.0369 .

IR (neat) 2964, 2362, 1680, 1621, 1562, 1482, 1434, 1352, 1319, 1153, 965, 915, 763, 671, 544.

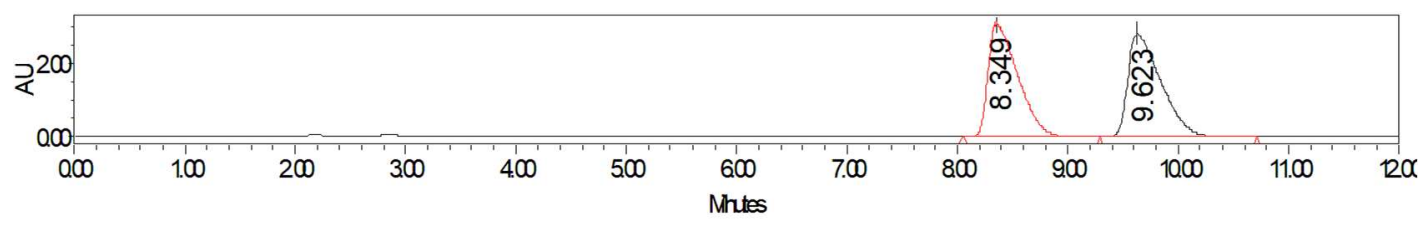

\begin{tabular}{|r|r|c|r|}
\hline & Retention Time & Area & \% Area \\
\hline 1 & 8.349 & 57107574 & 49.75 \\
\hline 2 & 9.623 & 57680939 & 50.25 \\
\hline
\end{tabular}

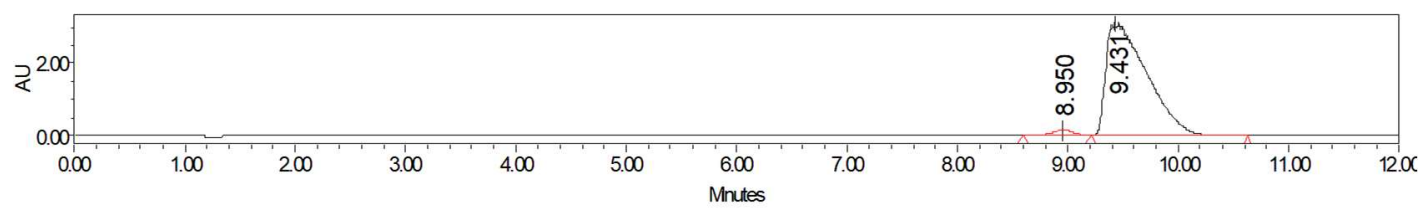

\begin{tabular}{|r|r|r|r|}
\hline & Retention Time & Area & \% Area \\
\hline 1 & 8.950 & 2445005 & 2.96 \\
\hline 2 & 9.431 & 80052026 & 97.04 \\
\hline
\end{tabular}


(E)-3-(2-Bromophenyl)-N-(2-(tert-butyl)phenyl)- $N$-(methylsulfonyl)acrylamide (3j):<smiles>CN(C(=O)/C=C/c1ccccc1Br)c1ccccc1C(C)(C)C</smiles>

3j

White solid. m.p. $50-54{ }^{\circ} \mathrm{C} .31 .8 \mathrm{mg}, 73 \%$ yield, $94 \%$ ee. Specific rotation $[\alpha]^{20}{ }_{\mathrm{D}}=+134.0\left(c=0.33, \mathrm{CH}_{2} \mathrm{Cl}_{2}\right)$. Dissolved in $i$-PrOH for HPLC; HPLC (Chiral ID column, $i-\mathrm{PrOH} / n$-hexane $=5 / 95$, flow rate: $1.0 \mathrm{~mL} / \mathrm{min}, 254 \mathrm{~nm}$ ): $\operatorname{tr}($ major $)=53.89 \mathrm{~min}, \operatorname{tr}(\operatorname{minor})=63.56$ min.

${ }^{1} \mathrm{H}$ NMR $\left(400 \mathrm{MHz}, \mathrm{CDCl}_{3}\right) \delta=8.17(\mathrm{~d}, J=15.4,1 \mathrm{H}), 7.70(\mathrm{dd}, J=8.2,1.5,1 \mathrm{H}), 7.61-7.55(\mathrm{~m}, 1 \mathrm{H}), 7.52-7.44(\mathrm{~m}, 1 \mathrm{H}), 7.30(\mathrm{td}$, $J=7.6,1.5,1 \mathrm{H}), 7.23-7.09(\mathrm{~m}, 4 \mathrm{H}), 6.12(\mathrm{~d}, J=15.5,1 \mathrm{H}), 3.59(\mathrm{~s}, 3 \mathrm{H}), 1.48(\mathrm{~s}, 9 \mathrm{H})$.

${ }^{13} \mathrm{C}\left\{{ }^{1} \mathrm{H}\right\}$ NMR $\left(101 \mathrm{MHz}, \mathrm{CDCl}_{3}\right) \delta 166.6,149.4,144.5,134.1,133.6,133.1,131.8,131.6,130.5,130.4,127.8127 .6,125.7,120.4$, $77.0,42.4,36.4,32.4$.

HRMS (ESI+) $\mathrm{m} / \mathbf{z}$ calcd for $\mathrm{C}_{20} \mathrm{H}_{22} \mathrm{BrNNaO}_{3} \mathrm{~S}[\mathrm{M}+\mathrm{Na}]^{+}: 458.0396$ and 460.0376 , found: 458.0388 and 460.0372 .

IR (neat) 2963, 2362, 1681, 1617, 1486, 1437, 1352, 1320, 1269, 1153, 1026, 965, 914, 857, 761, 546, 500.

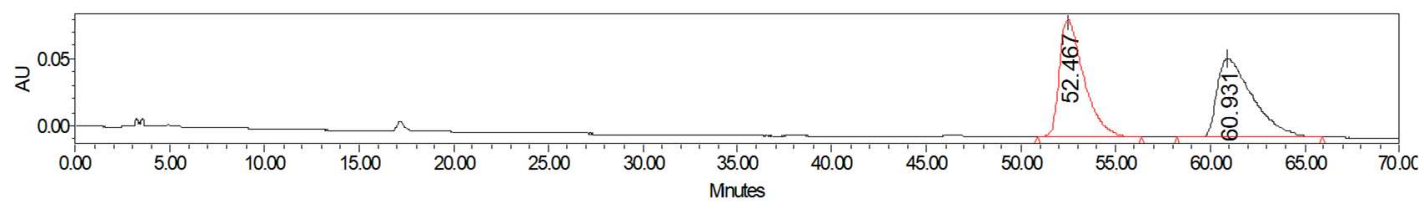

\begin{tabular}{|r|r|r|r|}
\hline & Retention Time & Area & \% Area \\
\hline 1 & 52.467 & 7718525 & 50.42 \\
\hline 2 & 60.931 & 7590514 & 49.58 \\
\hline
\end{tabular}

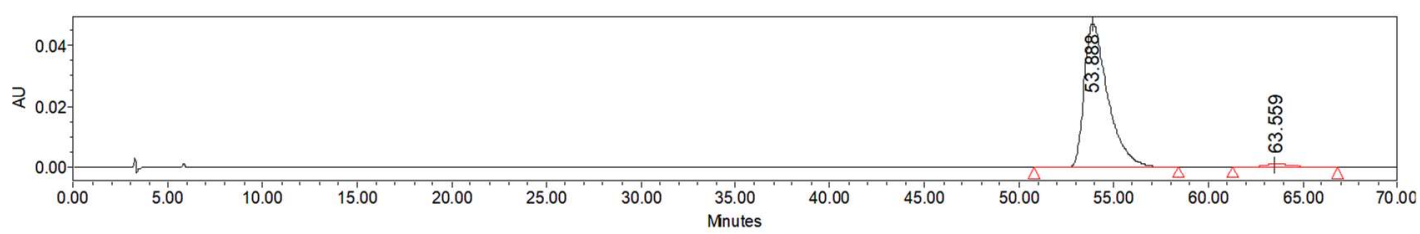

\begin{tabular}{|r|r|r|r|}
\hline & Retention Time & \multicolumn{1}{c|}{ Area } & \% Area \\
\hline 1 & 53.888 & 4119913 & 97.09 \\
\hline 2 & 63.559 & 123622 & 2.91 \\
\hline
\end{tabular}


(E)-N-(2-(Tert-butyl)phenyl)-N-(methylsulfonyl)-3-(4-(trifluoromethyl)phenyl)acrylamide (3k)<smiles>CC(C)(C)c1ccccc1N(C(=O)/C=C/c1ccc(C(F)(F)F)cc1)C(C)(C)C</smiles>

3k

White solid. m.p. $145-149{ }^{\circ} \mathrm{C}$. $41.6 \mathrm{mg},>95 \%$ yield, $94 \%$ ee. Specific rotation $[\alpha]^{20} \mathrm{D}=+92.9\left(c=1.00, \mathrm{CH}_{2} \mathrm{Cl}_{2}\right)$. Dissolved in $i-$ $\mathrm{PrOH}$ for HPLC; HPLC (Chiral ID column, $i-\mathrm{PrOH} / n$-hexane = 20/80, flow rate: $1.0 \mathrm{~mL} / \mathrm{min}, 254 \mathrm{~nm}$ ): $\operatorname{tr}($ major) $=12.09 \mathrm{~min}$, tr (minor) $=14.87 \mathrm{~min}$.

${ }^{1} \mathrm{H}$ NMR $\left(400 \mathrm{MHz}, \mathrm{CDCl}_{3}\right) \delta=7.81(\mathrm{~d}, J=15.5,1 \mathrm{H}), 7.72(\mathrm{dd}, J=8.2,1.5,1 \mathrm{H}), 7.56(\mathrm{~d}, J=8.1,2 \mathrm{H}), 7.50$ (ddd, $J=8.3,7.3,1.5$, $1 \mathrm{H}), 7.37$ (d, $J=8.1,2 \mathrm{H}), 7.31$ (td, $J=7.5,1.5,1 \mathrm{H}), 7.11(\mathrm{dd}, J=7.8,1.5,1 \mathrm{H}), 6.20(\mathrm{~d}, J=15.5,1 \mathrm{H}), 3.58(\mathrm{~s}, 3 \mathrm{H}), 1.48(\mathrm{~s}, 9 \mathrm{H})$.

${ }^{13} \mathrm{C}\left\{{ }^{1} \mathrm{H}\right\}$ NMR $\left(101 \mathrm{MHz}, \mathrm{CDCl}_{3}\right) \delta 166.6,149.4,144.2,137.3,133.0,132.2(\mathrm{~d}, J=33.3 \mathrm{~Hz}), 131.7,130.7,130.5,128.4,127.7,125.9$ (q, $J=4.0 \mathrm{~Hz}), 123.6(\mathrm{~d}, J=272.7 \mathrm{~Hz}), 120.1,42.4,36.4,32.3$.

${ }^{19} \mathrm{~F}\left\{{ }^{1} \mathrm{H}\right\}$ NMR $\left(376 \mathrm{MHz}, \mathrm{CDCl}_{3}\right) \delta-63.0$.

HRMS (ESI+) $\mathrm{m} / \mathrm{z}$ calcd for $\mathrm{C}_{21} \mathrm{H}_{22} \mathrm{~F}_{3} \mathrm{NNaO}_{3} \mathrm{~S}[\mathrm{M}+\mathrm{Na}]^{+}: 448.1165$, found: 448.1172 .

IR (neat) 2966, 2362, 1681, 1623, 1487, 1415, 1354, 1321, 1284, 1156, 1128, 1067, 1016, 964, 832, 763, 544.

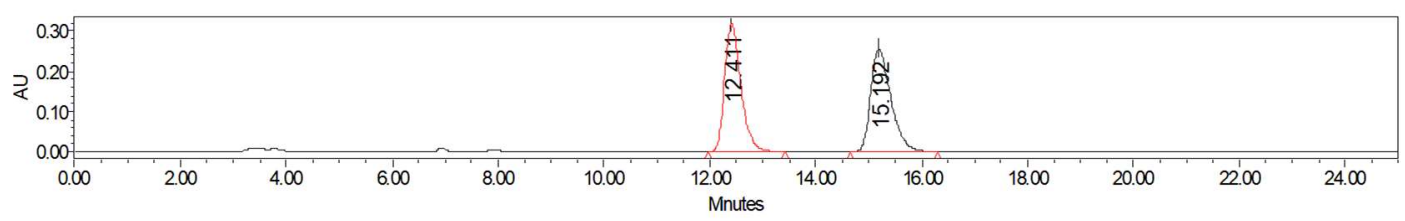

\begin{tabular}{|r|r|r|r|}
\hline & Retention Time & Area & \% Area \\
\hline 1 & 12.411 & 6771986 & 49.97 \\
\hline 2 & 15.192 & 6780265 & 50.03 \\
\hline
\end{tabular}

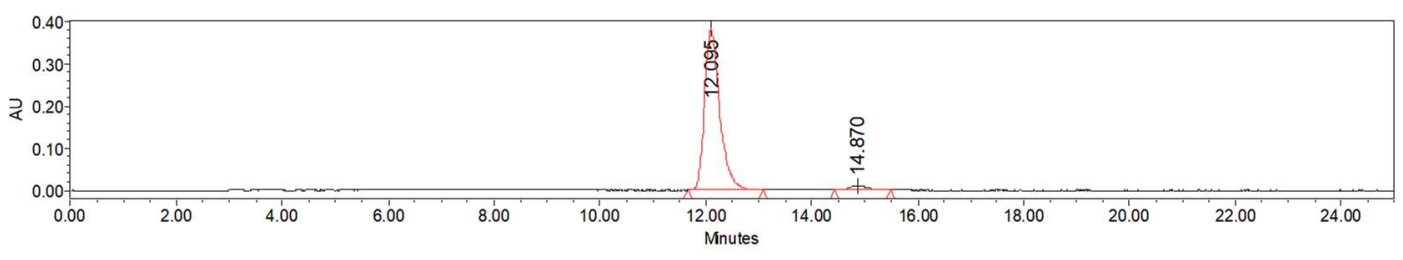

\begin{tabular}{|r|r|r|r|}
\hline & Retention Time & Area & \% Area \\
\hline 1 & 12.095 & 7647740 & 97.04 \\
\hline 2 & 14.870 & 233462 & 2.96 \\
\hline
\end{tabular}


(E)-N-(2-(Tert-butyl)phenyl)-3-(4-cyanophenyl)-N-(methylsulfonyl)acrylamide (3I)<smiles>CN(C(=O)/C=C/c1ccc(C#N)cc1)c1ccccc1Br</smiles>

3)

White solid. m.p. $202-205{ }^{\circ} \mathrm{C}$. $30.6 \mathrm{mg}, 80 \%$ yield, $95 \%$ ee. Specific rotation $[\alpha]^{20}{ }_{\mathrm{D}}=+104.2\left(c=0.50, \mathrm{CH}_{2} \mathrm{Cl}_{2}\right)$. Dissolved in $i-$ PrOH for HPLC; HPLC (Chiral ID column, $i-\mathrm{PrOH} / n$-hexane = 20/80, flow rate: $1.0 \mathrm{~mL} / \mathrm{min}, 254 \mathrm{~nm}$ ): $\operatorname{tr}($ major) $=46.53 \mathrm{~min}$, tr (minor) $=53.56 \mathrm{~min}$.

${ }^{1} \mathrm{H}$ NMR $\left(400 \mathrm{MHz}, \mathrm{CDCl}_{3}\right) \delta=7.77(\mathrm{~d}, J=15.6,1 \mathrm{H}), 7.72(\mathrm{dd}, J=8.2,1.5,1 \mathrm{H}), 7.62-7.57(\mathrm{~m}, 2 \mathrm{H}), 7.51$ (ddd, $J=8.3,7.3,1.5$, $1 \mathrm{H}), 7.37-7.28(\mathrm{~m}, 3 \mathrm{H}), 7.10(\mathrm{dd}, J=7.8,1.5,1 \mathrm{H}), 6.20(\mathrm{~d}, J=15.6,1 \mathrm{H}), 3.58(\mathrm{~s}, 3 \mathrm{H}), 1.47(\mathrm{~s}, 9 \mathrm{H})$.

${ }^{13} \mathrm{C}\left\{{ }^{1} \mathrm{H}\right\}$ NMR $\left(101 \mathrm{MHz}, \mathrm{CDCl}_{3}\right) \delta 166.3,149.4,143.6,138.1,132.9,132.7,131.7,130.8,130.5,128.6,127.8,120.9,118.1,113.8$, 42.4, 36.4, 32.3.

HRMS (ESI+) $\mathrm{m} / \mathrm{z}$ calcd for $\mathrm{C}_{21} \mathrm{H}_{22} \mathrm{~N}_{2} \mathrm{NaO}_{3} \mathrm{~S}[\mathrm{M}+\mathrm{Na}]^{+}: 405.1243$, found: 405.1233 .

IR (neat) 2964, 2362, 2229, 1681, 1623, 1486, 1437, 1486, 1437, 1353, 1323, 1279, 1154, 965, 914, 830, 763, $677,545$.

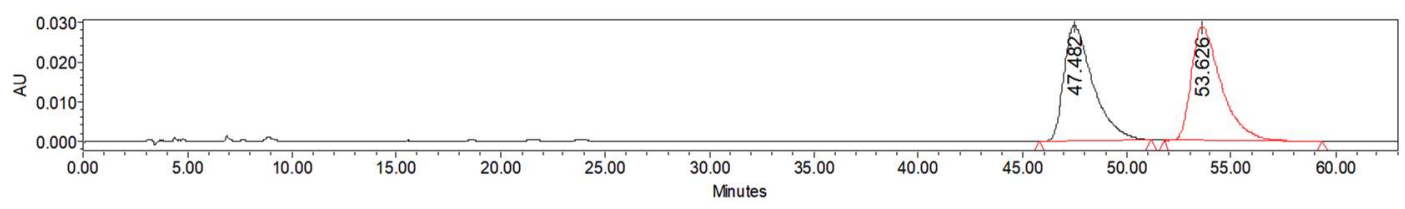

\begin{tabular}{|r|r|r|r|}
\hline & Retention Time & Area & \% Area \\
\hline 1 & 47.482 & 2824369 & 49.56 \\
\hline 2 & 53.626 & 2874638 & 50.44 \\
\hline
\end{tabular}

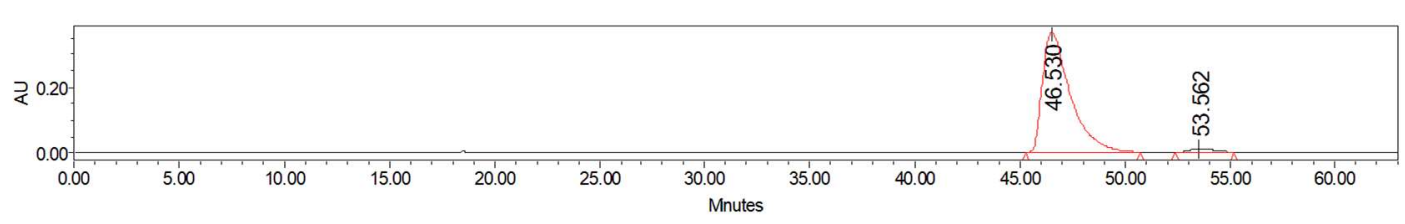

\begin{tabular}{|r|r|r|r|}
\hline & Retention Time & Area & \% Area \\
\hline 1 & 46.530 & 33968556 & 97.48 \\
\hline 2 & 53.562 & 878981 & 2.52 \\
\hline
\end{tabular}


(E)-N-(2-(Tert-butyl)phenyl)-N-(methylsulfonyl)-3-(4-nitrophenyl)acrylamide (3m)<smiles>CC(C)(C)c1ccccc1N(C(=O)/C=C/c1ccc([N+](=O)[O-])cc1)C(C)(C)C</smiles>

Yellew solid. m.p. $153-157^{\circ} \mathrm{C} .39 .4 \mathrm{mg},>95 \%$ yield, $93 \%$ ee. Specific rotation $[\alpha]^{20}{ }_{\mathrm{D}}=+105.6\left(c=0.55, \mathrm{CH}_{2} \mathrm{Cl}_{2}\right)$. Dissolved in $i-$ PrOH for SFC; SFC (Chiralcel C1, $\mathrm{CO}_{2} / \mathrm{i}-\mathrm{PrOH}=80 / 20$, flow rate $=1.0 \mathrm{~mL} / \mathrm{min}, 254 \mathrm{~nm}$ ): $\operatorname{tr}(\operatorname{minor})=13.91 \mathrm{~min}, \operatorname{tr}($ major $)=16.93 \mathrm{~min}$. ${ }^{1} \mathrm{H}$ NMR $\left(400 \mathrm{MHz}, \mathrm{CDCl}_{3}\right) \delta=8.16(\mathrm{~d}, J=8.5,2 \mathrm{H}), 7.82(\mathrm{~d}, J=15.6,1 \mathrm{H}), 7.73(\mathrm{dd}, J=8.2,1.4,1 \mathrm{H}), 7.55-7.48(\mathrm{~m}, 1 \mathrm{H}), 7.41(\mathrm{~d}$, $J=8.5,2 \mathrm{H}), 7.32(\mathrm{td}, J=7.6,1.5,1 \mathrm{H}), 7.11(\mathrm{dd}, J=7.8,1.4,1 \mathrm{H}), 6.24(\mathrm{~d}, J=15.5,1 \mathrm{H}), 3.58(\mathrm{~s}, 3 \mathrm{H}), 1.47(\mathrm{~s}, 9 \mathrm{H})$.

${ }^{13} \mathrm{C}\left\{{ }^{1} \mathrm{H}\right\}$ NMR $\left(101 \mathrm{MHz}, \mathrm{CDCl}_{3}\right) \delta 166.2,149.4,148.7,143.0,139.9,132.8,131.7,130.8,130.6,128.8,127.8,124.2,121.7,42.4$, 36.4, 32.3.

HRMS (ESI+) $\mathrm{m} / \mathrm{z}$ calcd for $\mathrm{C}_{21} \mathrm{H}_{26} \mathrm{~N}_{2} \mathrm{NaO}_{5} \mathrm{~S}[\mathrm{M}+\mathrm{Na}]^{+}: 425.1142$, found: 425.1135 .

IR (neat) 3729, 2966, 2362, 2336, 1682, 1624, 1521, 1349, 1275, 1155, 965, 841, 758, 675, 544.

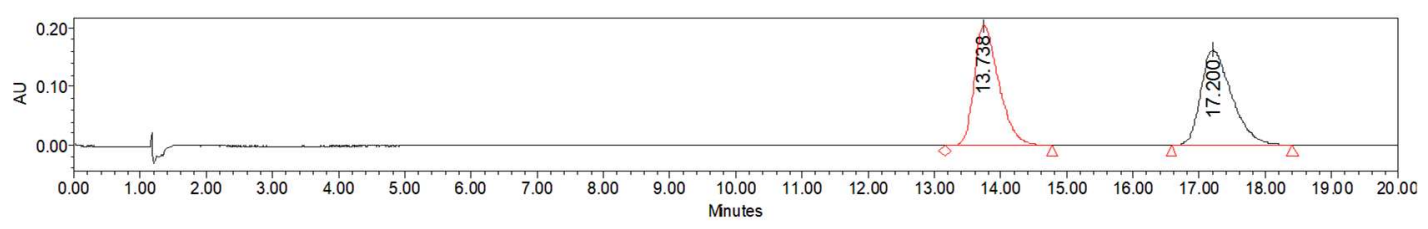

\begin{tabular}{|r|r|r|r|}
\hline & Retention Time & Area & \% Area \\
\hline 1 & 13.733 & 12099533 & 50.22 \\
\hline 2 & 17.200 & 11995884 & 49.78 \\
\hline
\end{tabular}

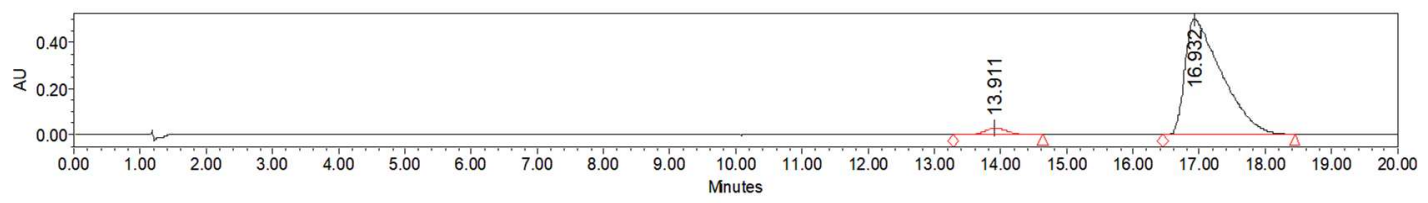

\begin{tabular}{|r|r|r|r|}
\hline & Retention Time & Area & \% Area \\
\hline 1 & 13.911 & 760578 & 3.75 \\
\hline 2 & 16.932 & 19525480 & 96.25 \\
\hline
\end{tabular}


(E)-N-(2-(Tert-butyl)phenyl)-N-(methylsulfonyl)-3-(naphthalen-1-yl)acrylamide (3n):<smiles>CN(C(=O)/C=C/c1cccc2ccccc12)c1ccccc1C(C)(C)C</smiles>

$3 n$

White solid. m.p. $63-67^{\circ} \mathrm{C} .35 .1 \mathrm{mg}, 86 \%$ yield, $95 \%$ ee. Specific rotation $[\alpha]^{20} \mathrm{D}=+167.5\left(c=0.46, \mathrm{CH}_{2} \mathrm{Cl}_{2}\right)$. Dissolved in $i-\mathrm{PrOH}$ for HPLC; HPLC (Chiral IA column, $i-\mathrm{PrOH} / n$-hexane = 20/80, flow rate: $1.0 \mathrm{~mL} / \mathrm{min}, 254 \mathrm{~nm}$ ): $\operatorname{tr}(\operatorname{minor})=11.56 \mathrm{~min}$, $\operatorname{tr}(\mathrm{major})=14.54$ min.

${ }^{1} \mathrm{H}$ NMR $\left(400 \mathrm{MHz}, \mathrm{CDCl}_{3}\right) \delta=8.66(\mathrm{~d}, J=15.3,1 \mathrm{H}), 8.18-8.10(\mathrm{~m}, 1 \mathrm{H}), 7.85(\mathrm{ddd}, J=7.2,5.6,1.6,2 \mathrm{H}), 7.71(\mathrm{dd}, J=8.2,1.5$, $1 \mathrm{H}), 7.61-7.45(\mathrm{~m}, 3 \mathrm{H}), 7.41-7.28(\mathrm{~m}, 3 \mathrm{H}), 7.16(\mathrm{dd}, J=7.8,1.5,1 \mathrm{H}), 6.23(\mathrm{~d}, J=15.3,1 \mathrm{H}), 3.63(\mathrm{~s}, 3 \mathrm{H}), 1.51(\mathrm{~s}, 9 \mathrm{H})$.

${ }^{13} \mathrm{C}\left\{{ }^{1} \mathrm{H}\right\}$ NMR $\left(101 \mathrm{MHz}, \mathrm{CDCl}_{3}\right) \delta 167.1,149.5,143.3,133.6,133.2,131.8,131.5,131.4,131.1,130.5,130.4,128.7,127.6,127.1$, $126.4,125.3,125.2,123.3,120.4,42.4,36.4,32.4$.

HRMS (ESI+) $\mathrm{m} / \mathrm{z}$ calcd for $\mathrm{C}_{24} \mathrm{H}_{25} \mathrm{NNaO}_{3} \mathrm{~S}[\mathrm{M}+\mathrm{Na}]^{+}: 446.1187$, found: 446.1188 .

IR (neat) 2963, 2360, 1678, 1611, 1487, 1437, 1397, 1349, 1308, 1252, 1153, 1089, 1053, 965, 852, 798, 767, 736, $540,496$.

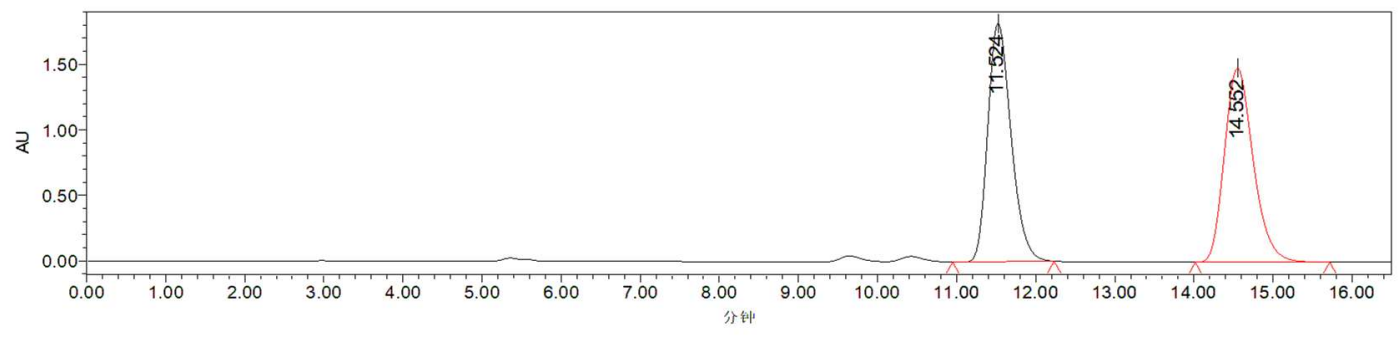

\begin{tabular}{|r|r|r|r|}
\hline & Retention Time & Area & \% Area \\
\hline 1 & 11.524 & 37558005 & 49.89 \\
\hline 2 & 14.552 & 37719668 & 50.11 \\
\hline
\end{tabular}

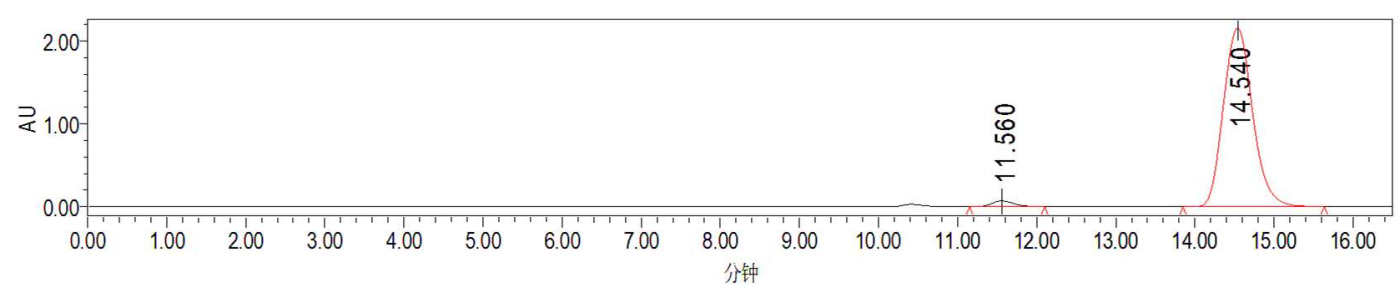

\begin{tabular}{|r|r|r|r|}
\hline & Retention Time & Area & \% Area \\
\hline 1 & 11.560 & 1399779 & 2.47 \\
\hline 2 & 14.540 & 55162375 & 97.53 \\
\hline
\end{tabular}


<smiles>CN(C(=O)/C=C/c1ccco1)c1ccccc1C(C)(C)C</smiles>

30

Brown solid. m.p. $63-68^{\circ} \mathrm{C}$. $25.7 \mathrm{mg}, 74 \%$ yield, $95 \%$ ee. Specific rotation $[\alpha]^{20}{ }_{\mathrm{D}}=+125.0\left(c=0.22, \mathrm{CH}_{2} \mathrm{Cl}_{2}\right)$. Dissolved in $i$-PrOH for SFC; SFC (Chiralcel C1, $\mathrm{CO}_{2} / i-\mathrm{PrOH}=80 / 20$, flow rate $\left.=1.0 \mathrm{~mL} / \mathrm{min}, 254 \mathrm{~nm}\right): \operatorname{tr}($ minor $)=6.67 \mathrm{~min}, \operatorname{tr}($ major $)=7.11 \mathrm{~min}$.

${ }^{1} \mathrm{H}$ NMR $\left(400 \mathrm{MHz}, \mathrm{CDCl}_{3}\right) \delta=7.69(\mathrm{dd}, J=8.2,1.5,1 \mathrm{H}), 7.54(\mathrm{~d}, J=15.2,1 \mathrm{H}), 7.47(\mathrm{ddd}, J=8.4,7.3,1.5,1 \mathrm{H}), 7.39-7.25(\mathrm{~m}$, $3 \mathrm{H}), 7.08$ (dd, $J=7.8,1.5,1 \mathrm{H}), 6.61(\mathrm{~d}, J=3.4,1 \mathrm{H}), 6.42(\mathrm{dd}, J=3.5,1.8,1 \mathrm{H}), 5.99(\mathrm{~d}, J=15.2,1 \mathrm{H}), 3.56(\mathrm{~s}, 3 \mathrm{H}), 1.47(\mathrm{~s}, 9 \mathrm{H})$.

${ }^{13} \mathrm{C}\left\{{ }^{1} \mathrm{H}\right\}$ NMR $\left(101 \mathrm{MHz}, \mathrm{CDCl}_{3}\right) \delta 167.3,150.8,149.5,145.3,133.2,132.1,131.7,130.4,130.4,127.5,116.6,115.1,112.5,42.3$, 36.4, 32.3.

HRMS (ESI+) $\mathrm{m} / \mathrm{z}$ calcd for $\mathrm{C}_{18} \mathrm{H}_{21} \mathrm{NNaO}_{4} \mathrm{~S}[\mathrm{M}+\mathrm{Na}]^{+}: 370.1083$, found: 370.1082 .

IR (neat) 2964, 2362, 1677, 1617, 1554, 1482, 1351, 1314, 1272, 1208, 1151, 1019, 966, 820, 758, 545.

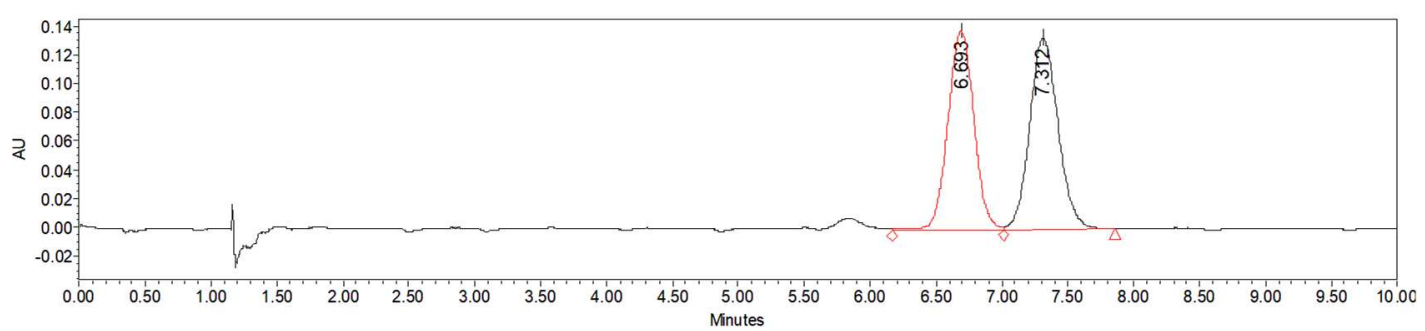

\begin{tabular}{|r|r|r|r|}
\hline & Retention Time & Area & \% Area \\
\hline 1 & 6.693 & 1913274 & 48.97 \\
\hline 2 & 7.312 & 1994113 & 51.03 \\
\hline
\end{tabular}

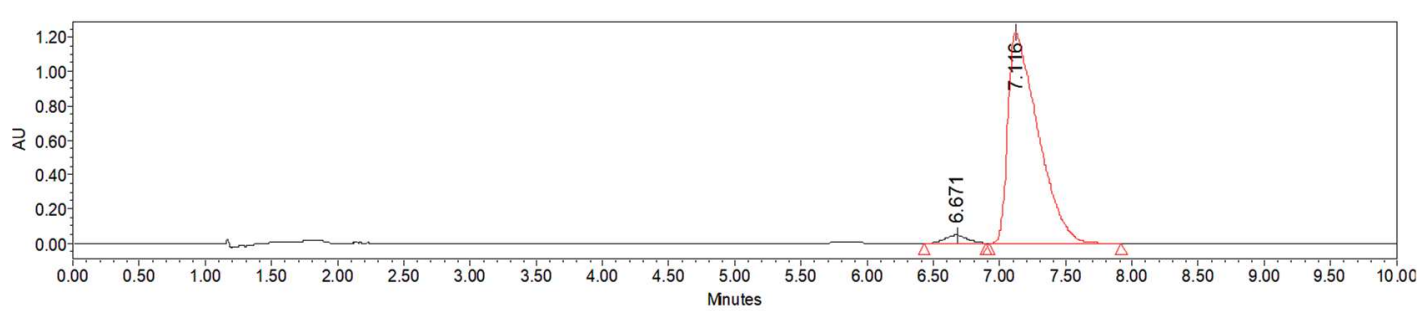

\begin{tabular}{|r|r|r|r|}
\hline & Retention Time & Area & \% Area \\
\hline 1 & 6.671 & 527619 & 2.65 \\
\hline 2 & 7.116 & 19374171 & 97.35 \\
\hline
\end{tabular}


(E)-N-(2-(Tert-butyl)phenyl)-N-(methylsulfonyl)-3-(thiophen-2-yl)acrylamide (3p)<smiles>CN(C(=O)/C=C/c1cccs1)c1ccccc1C(C)(C)C</smiles>

$3 p$

White solid. m.p. $55-62{ }^{\circ} \mathrm{C} .25 .4 \mathrm{mg}, 70 \%$ yield, $96 \%$ ee. Specific rotation $[\alpha]^{20}{ }_{\mathrm{D}}=+162.1\left(c=0.27, \mathrm{CH}_{2} \mathrm{Cl}_{2}\right)$. Dissolved in $i-\mathrm{PrOH}$ for HPLC; HPLC (Chiral ID column, $i-\mathrm{PrOH} / n$-hexane = 20/80, Flow rate: $1.0 \mathrm{~mL} / \mathrm{min}, 254 \mathrm{~nm}$ ): $\operatorname{tr}$ (major) $=25.66 \mathrm{~min}, \operatorname{tr}(\operatorname{minor})=33.55$ $\min$.

${ }^{1} \mathrm{H}$ NMR $\left(400 \mathrm{MHz}, \mathrm{CDCl}_{3}\right) \delta=7.89(\mathrm{~d}, J=15.2,1 \mathrm{H}), 7.69(\mathrm{dd}, J=8.2,1.5,1 \mathrm{H}), 7.48(\mathrm{ddd}, J=8.2,7.2,1.5,1 \mathrm{H}), 7.35-7.26(\mathrm{~m}$, 2H), $7.23-7.16(\mathrm{~m}, 1 \mathrm{H}), 7.05$ (ddd, $J=32.2,6.5,2.6,2 \mathrm{H}), 5.90(\mathrm{~d}, J=15.2,1 \mathrm{H}), 3.56(\mathrm{~s}, 3 \mathrm{H}), 1.47(\mathrm{~s}, 9 \mathrm{H})$.

${ }^{13} \mathrm{C}\left\{{ }^{1} \mathrm{H}\right\}$ NMR $\left(101 \mathrm{MHz}, \mathrm{CDCl}_{3}\right) \delta 167.0,149.4,139.4,138.5,133.1,132.0,131.7,130.5,129.3,128.4,128.6,116.4,42.4,36.4$, 32.4 .

HRMS (ESI+) $\mathrm{m} / \mathrm{z}$ calcd for $\mathrm{C}_{18} \mathrm{H}_{21} \mathrm{NNaO}_{3} \mathrm{~S}_{2}[\mathrm{M}+\mathrm{Na}]^{+}: 386.0855$, found: 386.0862 .

IR (neat) 2963, 2362, 1675, 1605, 1486, 1426, 1351, 1315, 1278, 1204, 1151, 1048, 965, 916, 833, 763, 543.

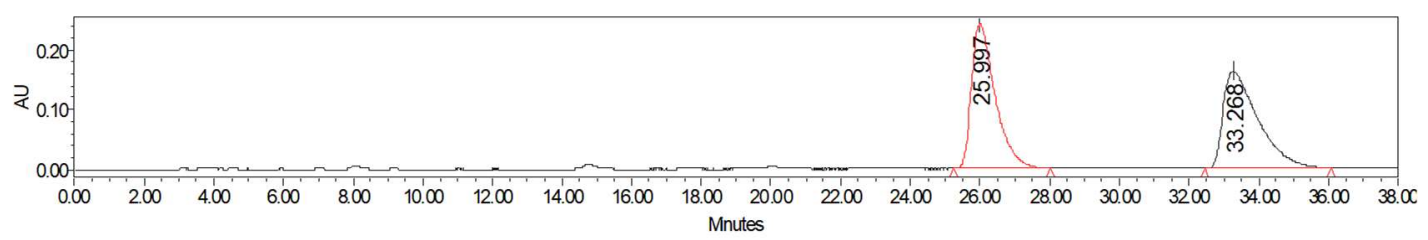

\begin{tabular}{|r|r|r|r|}
\hline & Retention Time & Area & \% Area \\
\hline 1 & 25.997 & 11498086 & 50.19 \\
\hline 2 & 33.268 & 11412442 & 49.81 \\
\hline
\end{tabular}

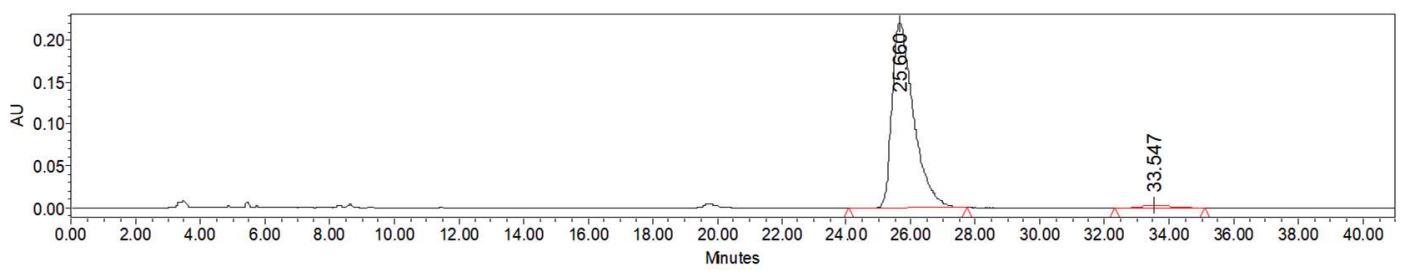

\begin{tabular}{|r|r|r|r|}
\hline & Retention Time & Area & \% Area \\
\hline 1 & 25.660 & 10199279 & 97.82 \\
\hline 2 & 33.547 & 227599 & 2.18 \\
\hline
\end{tabular}


(E)-N-(2-(tert-butyl)phenyl)-4-methyl- $N$-(methylsulfonyl)pent-2-enamide (3q)<smiles>CC(C)/C=C/C(=O)N(C)c1ccccc1Br</smiles>

$3 q$

Colorless oil. The yield was determined by NMR using $\mathrm{CH}_{2} \mathrm{Br}_{2}(0.1 \mathrm{mmol})$ as an internal standard. $61 \%$ yield, $98 \%$ ee. Dissolved in $i$-PrOH for HPLC; HPLC (Chiral ID column, $i-\mathrm{PrOH} / n$-hexane = 5/95, Flow rate: $1.0 \mathrm{~mL} / \mathrm{min}, 254 \mathrm{~nm}$ ): $\operatorname{tr}(\mathrm{major})=29.65 \mathrm{~min}$, tr (minor) $=33.41 \mathrm{~min}$.

${ }^{1} \mathrm{H}$ NMR $\left(400 \mathrm{MHz}, \mathrm{CDCl}_{3}\right) \delta=7.65(\mathrm{~d}, J=9.0,1 \mathrm{H}), 7.44(\mathrm{td}, J=8.2,7.7,1.5,1 \mathrm{H}), 7.26(\mathrm{td}, J=7.6,1.6,1 \mathrm{H}), 7.11-6.99(\mathrm{~m}, 2 \mathrm{H})$, $5.47(\mathrm{~d}, J=15.3,1 \mathrm{H}), 3.53(\mathrm{~s}, 3 \mathrm{H}), 1.44(\mathrm{~s}, 9 \mathrm{H}), 0.91(\mathrm{~d}, J=6.8,6 \mathrm{H})$.

${ }^{13} \mathrm{C}\left\{{ }^{1} \mathrm{H}\right\}$ NMR $\left(101 \mathrm{MHz}, \mathrm{CDCl}_{3}\right) \delta 167.3,157.7,149.3,133.2,131.6,130.3,130.3,127.4,118.7,42.3,32.3,31.2,21.1,21.0$.

HRMS (ESI+) $\mathrm{m} / \mathrm{z}$ calcd for $\mathrm{C}_{17} \mathrm{H}_{25} \mathrm{NNaO}_{3} \mathrm{~S}[\mathrm{M}+\mathrm{Na}]^{+}: 346.1447$, found: 346.1445 .

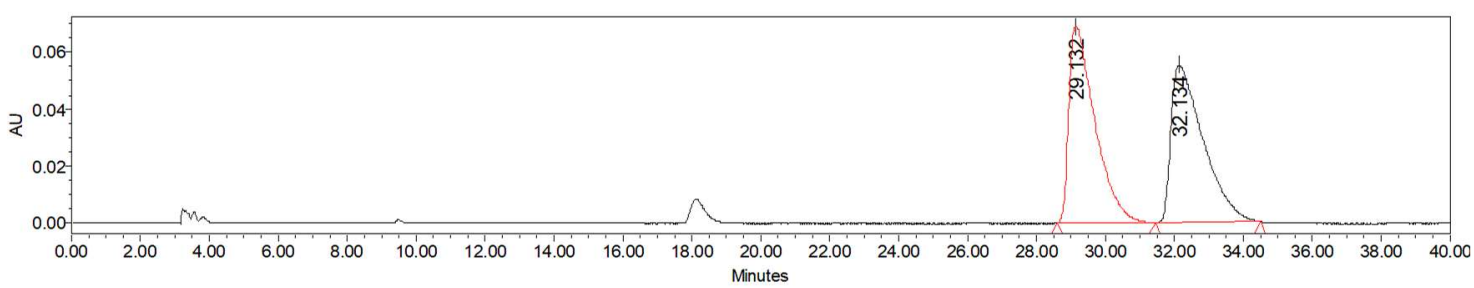

\begin{tabular}{|r|r|r|r|}
\hline & Retention Time & Area & \% Area \\
\hline 1 & 29.132 & 3632132 & 50.21 \\
\hline 2 & 32.134 & 3601039 & 49.79 \\
\hline
\end{tabular}

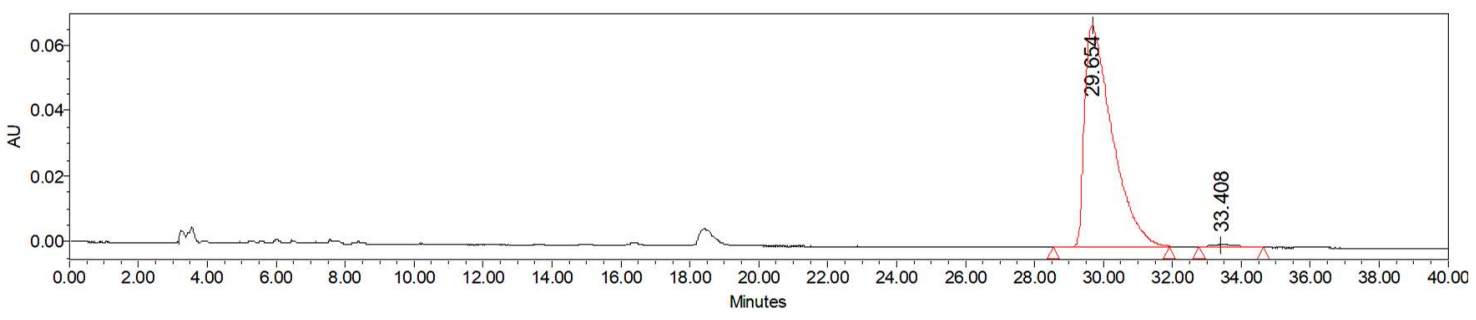

\begin{tabular}{|r|r|r|r|}
\hline & Retention Time & \multicolumn{1}{c|}{ Area } & \multicolumn{1}{c|}{$\%$ Area } \\
\hline 1 & 29.654 & 3776417 & 98.81 \\
\hline 2 & 33.408 & 45654 & 1.19 \\
\hline
\end{tabular}


(E)-N-(2-(tert-butyl)phenyl)- $N$-(methylsulfonyl)pent-2-enamide (3r)<smiles>CC/C=C/C(=O)N(C)c1ccccc1Br</smiles>

$3 r$

Colorless oil. The yield was determined by NMR using $\mathrm{CH}_{2} \mathrm{Br}_{2}(0.1 \mathrm{mmol})$ as an internal standard. 95\% yield, $94 \%$ ee. Dissolved in $i-\mathrm{PrOH}$ for HPLC; HPLC (Chiral ID column, $i-\mathrm{PrOH} / n$-hexane $=5 / 95$, Flow rate: $1.0 \mathrm{~mL} / \mathrm{min}, 254 \mathrm{~nm}$ ): $\operatorname{tr}(\operatorname{major})=38.15 \mathrm{~min}, \operatorname{tr}(\mathrm{minor})$ $=43.67 \mathrm{~min}$.

${ }^{1} \mathrm{H}$ NMR $\left(400 \mathrm{MHz}, \mathrm{CDCl}_{3}\right) \delta=7.65(\mathrm{dt}, J=8.2,1.2,1 \mathrm{H}), 7.44(\mathrm{tt}, J=8.3,1.3,1 \mathrm{H}), 7.27-7.11(\mathrm{~m}, 2 \mathrm{H}), 7.04(\mathrm{dt}, J=7.9,1.2,1 \mathrm{H})$, $5.52(\mathrm{dq}, J=15.2,1.5,1 \mathrm{H}), 3.52(\mathrm{~d}, J=0.9,3 \mathrm{H}), 2.16-2.06(\mathrm{~m}, 2 \mathrm{H}), 1.44(\mathrm{~d}, J=1.0,9 \mathrm{H}), 0.90(\mathrm{td}, J=7.4,0.9,3 \mathrm{H})$.

${ }^{13} \mathrm{C}\left\{{ }^{1} \mathrm{H}\right\}$ NMR $\left(101 \mathrm{MHz}, \mathrm{CDCl}_{3}\right) \delta 167.1,153.0,149.3,133.2,131.6,130.3,130.3,127.4,120.4,42.3,36.3,32.3,25.6,12.0$.

HRMS (ESI+) $\mathrm{m} / \mathrm{z}$ calcd for $\mathrm{C}_{16} \mathrm{H}_{23} \mathrm{NNaO}_{3} \mathrm{~S}[\mathrm{M}+\mathrm{Na}]^{+}: 332.1291$, found: 332.1289 .

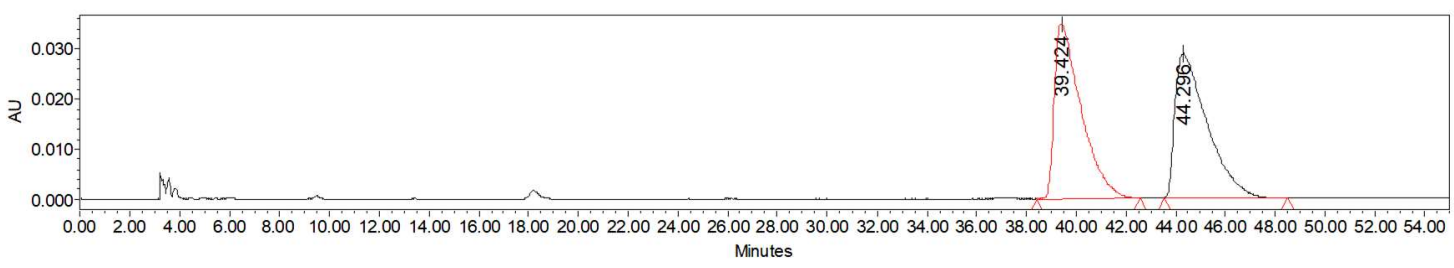

\begin{tabular}{|r|r|r|r|}
\hline & Retention Time & Area & \% Area \\
\hline 1 & 39.424 & 2618148 & 50.29 \\
\hline 2 & 44.296 & 2587742 & 49.71 \\
\hline
\end{tabular}

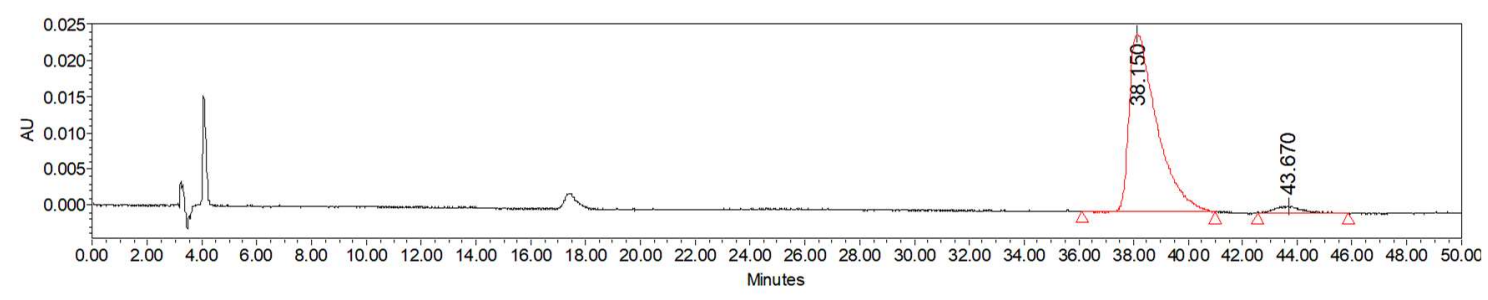

\begin{tabular}{|r|r|r|r|}
\hline & Retention Time & Area & \multicolumn{1}{c|}{$\%$ Area } \\
\hline 1 & 38.150 & 1727272 & 96.76 \\
\hline 2 & 43.670 & 57904 & 3.24 \\
\hline
\end{tabular}


<smiles>CC(C)(C)c1cc([N+](=O)[O-])ccc1N(C(=O)/C=C/c1ccccc1)C(=O)c1ccccc1</smiles>

3s

White solid. m.p. $73-75^{\circ} \mathrm{C} .31 .9 \mathrm{mg}, 80 \%$ yield, $87 \%$ ee. Specific rotation $[\alpha]^{20}{ }_{\mathrm{D}}=-96.4\left(c=0.27, \mathrm{CH}_{2} \mathrm{Cl}_{2}\right)$. Dissolved in $i-\mathrm{PrOH}$ for SFC; SFC (Chiralcel C1, $\mathrm{CO}_{2} / i-\mathrm{PrOH}=80 / 20$, flow rate $=1.0 \mathrm{~mL} / \mathrm{min}, 254 \mathrm{~nm}$ ): $\operatorname{tr}($ minor $)=5.27 \mathrm{~min}, \operatorname{tr}(\mathrm{major})=6.05 \mathrm{~min}$.

${ }^{1} \mathrm{H} \mathrm{NMR}\left(400 \mathrm{MHz}, \mathrm{CDCl}_{3}\right) \delta=8.30(\mathrm{dd}, J=9.0,2.5,1 \mathrm{H}), 7.94(\mathrm{dd}, J=19.4,2.6,2 \mathrm{H}), 7.88(\mathrm{~d}, J=9.1,1 \mathrm{H}), 7.42-7.29(\mathrm{~m}, 5 \mathrm{H}), 6.17$ $(\mathrm{d}, J=15.4,1 \mathrm{H}), 3.60(\mathrm{~s}, 3 \mathrm{H}), 1.52(\mathrm{~s}, 9 \mathrm{H})$.

${ }^{13} \mathrm{C}\left\{{ }^{1} \mathrm{H}\right\}$ NMR $\left(101 \mathrm{MHz}, \mathrm{CDCl}_{3}\right) \delta 166.2,157.6,148.0,146.4,134.4,133.6,131.7,131.4,129.1,128.5,126.6,124.7,116.5,42.7$, $37.4,32.1$.

HRMS (ESI+) $\mathrm{m} / \mathrm{z}$ calcd for $\mathrm{C}_{21} \mathrm{H}_{26} \mathrm{~N}_{2} \mathrm{NaO}_{5} \mathrm{~S}[\mathrm{M}+\mathrm{Na}]^{+}: 425.1142$, found: 425.1152 .

IR (neat) 2968, 2362, 1682, 1617, 1521, 1486, 1397, 1351, 1279, 1228, 1151, 1076, 965, 842, 762, 704, 546, 518.

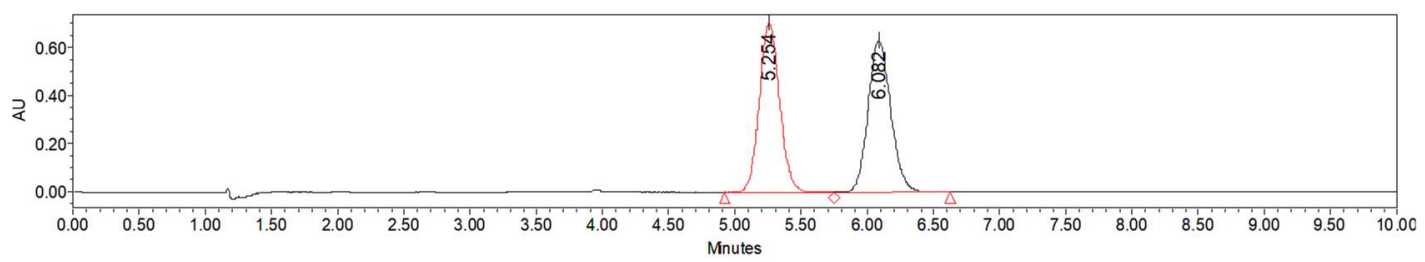

\begin{tabular}{|r|r|r|r|}
\hline & Retention Time & Area & \% Area \\
\hline 1 & 5.254 & 7514796 & 49.99 \\
\hline 2 & 6.082 & 7517921 & 50.01 \\
\hline
\end{tabular}

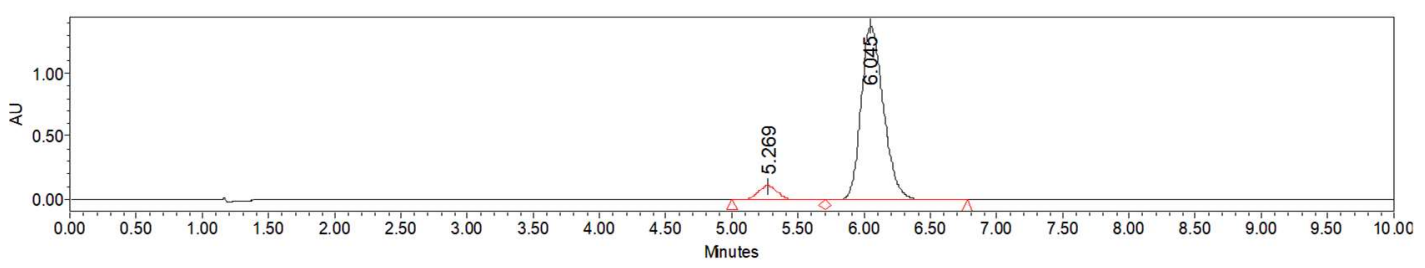

\begin{tabular}{|r|r|r|r|}
\hline & Retention Time & Area & $\%$ Area \\
\hline 1 & 5.269 & 1131285 & 6.54 \\
\hline 2 & 6.045 & 16160269 & 93.46 \\
\hline
\end{tabular}


$N$-(4-Bromo-2-(tert-butyl)phenyl)- $N$-(methylsulfonyl)cinnamamide (3t)<smiles>CN(C(=O)/C=C/c1ccccc1)c1ccc(Br)cc1C(C)(C)C</smiles>

$3 t$

White solid. m.p. $142-145^{\circ} \mathrm{C}$. $31.0 \mathrm{mg}, 71 \%$ yield, $95 \%$ ee. Specific rotation $[\alpha]^{20}{ }_{D}=+60.8\left(c=0.48, \mathrm{CH}_{2} \mathrm{Cl}_{2}\right)$. Dissolved in $i-\mathrm{PrOH}$ for HPLC; HPLC (Chiral IA column, $i$-PrOH $/ n$-hexane = 10/90, flow rate: $1.0 \mathrm{~mL} / \mathrm{min}, 254 \mathrm{~nm}$ ): $\operatorname{tr}(\operatorname{minor})=18.25 \mathrm{~min}, \operatorname{tr}(\mathrm{major})=24.79$ min.

${ }^{1} \mathrm{H}$ NMR $\left(400 \mathrm{MHz}, \mathrm{CDCl}_{3}\right) \delta=7.86-7.80(\mathrm{~m}, 2 \mathrm{H}), 7.43(\mathrm{dd}, J=8.3,2.3,1 \mathrm{H}), 7.39-7.29(\mathrm{~m}, 5 \mathrm{H}), 6.99(\mathrm{~d}, J=8.3,1 \mathrm{H}), 6.13(\mathrm{~d}, J$ $=15.5,1 \mathrm{H}), 3.58(\mathrm{~s}, 3 \mathrm{H}), 1.46(\mathrm{~s}, 9 \mathrm{H})$.

${ }^{13} \mathrm{C}\left\{{ }^{1} \mathrm{H}\right\}$ NMR $\left(101 \mathrm{MHz}, \mathrm{CDCl}_{3}\right) \delta 166.8,151.7,147.0,133.8,133.7,133.3,132.4,131.1,130.9,129.0,128.4,124.9,117.1,42.6$, 36.6, 32.1 .

HRMS (ESI+) $\mathrm{m} / \mathrm{z}$ calcd for $\mathrm{C}_{20} \mathrm{H}_{22} \mathrm{BrNNaO}_{3} \mathrm{~S}[\mathrm{M}+\mathrm{Na}]^{+}: 458.0396$ and 460.0376 , found: 458.0391 and 460.0369 .

IR (neat) 2965, 2362, 1679, 1617, 1482, 1352, 1276, 1229, 1152, 1088, 965, 913, 850, 764, 705, 542, 485.

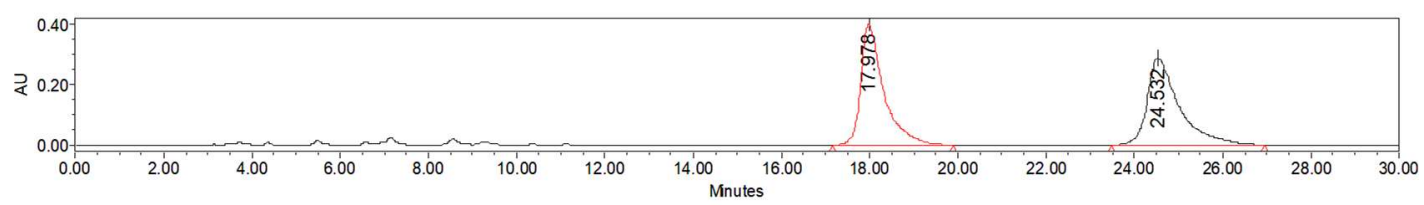

\begin{tabular}{|l|r|r|r|}
\hline & Retention Time & Area & \% Area \\
\hline 1 & 17.978 & 14317727 & 50.01 \\
\hline 2 & 24.532 & 14310565 & 49.99 \\
\hline
\end{tabular}

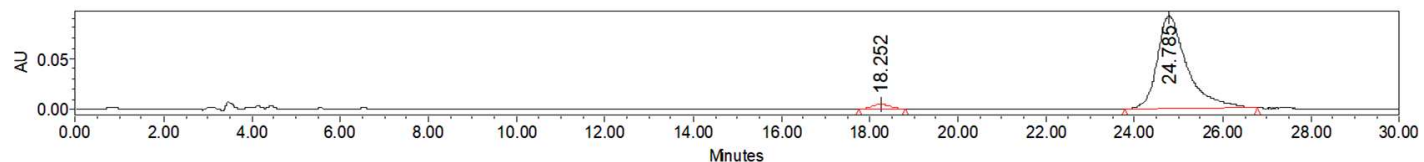

\begin{tabular}{|r|r|r|r|}
\hline & Retention Time & Area & \% Area \\
\hline 1 & 18.252 & 123249 & 2.77 \\
\hline 2 & 24.785 & 4328415 & 97.23 \\
\hline
\end{tabular}


<smiles>CN(C(=O)/C=C/c1ccccc1)c1ccc(I)cc1C(C)(C)C</smiles>

$3 \mathbf{u}$

White solid. m.p. $153-157^{\circ} \mathrm{C}$. $30.6 \mathrm{mg}, 63 \%$ yield, $92 \%$ ee. Specific rotation $[\alpha]^{20}{ }_{\mathrm{D}}=+44.2\left(\mathrm{c}=0.39, \mathrm{CH}_{2} \mathrm{Cl}_{2}\right)$. Dissolved in $i-\mathrm{PrOH}$ for HPLC; HPLC (Chiral IA column, $i$-PrOH $/ n$-hexane = 20/80, flow rate: $1.0 \mathrm{~mL} / \mathrm{min}, 254 \mathrm{~nm}$ ): $\operatorname{tr}(\operatorname{minor})=18.49 \mathrm{~min}, \operatorname{tr}(\mathrm{major})=24.68$ $\min$.

${ }^{1} \mathrm{H}$ NMR $\left(400 \mathrm{MHz}, \mathrm{CDCl}_{3}\right) \delta=8.00(\mathrm{~d}, J=2.0,1 \mathrm{H}), 7.83(\mathrm{~d}, J=15.5,1 \mathrm{H}), 7.62(\mathrm{dd}, J=8.3,2.0,1 \mathrm{H}), 7.42-7.28(\mathrm{~m}, 5 \mathrm{H}), 6.83(\mathrm{~d}$, $J=8.2,1 \mathrm{H}), 6.14(\mathrm{~d}, J=15.4,1 \mathrm{H}), 3.57(\mathrm{~s}, 3 \mathrm{H}), 1.45(\mathrm{~s}, 9 \mathrm{H})$.

${ }^{13} \mathrm{C}\left\{{ }^{1} \mathrm{H}\right\}$ NMR $\left(101 \mathrm{MHz}, \mathrm{CDCl}_{3}\right) \delta 166.8,151.7,147.0,139.7,136.9,133.8,133.3,133.1,131.1,129.0,128.4,117.2,97.2,42.6$, 36.5, 32.1 .

HRMS (ESI+) $\mathrm{m} / \mathrm{z}$ calcd for $\mathrm{C}_{20} \mathrm{H}_{23} \mathrm{INO}_{3} \mathrm{~S}[\mathrm{M}+\mathrm{H}]^{+}:$: 484.0438, found: 484.0431 .

IR (neat) 3729, 2964, 2361, 2336, 1680, 1618, 1577, 1481, 1353, 1276, 1228, 1153, 1078, 965, 912, 962, 679, 542.

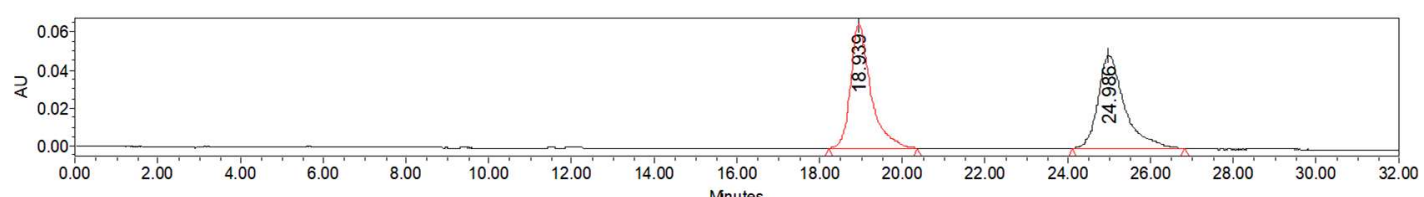

\begin{tabular}{|r|r|r|r|}
\hline & Retention Time & Area & \% Area \\
\hline 1 & 18.939 & 2221664 & 50.19 \\
\hline 2 & 24.986 & 2205078 & 49.81 \\
\hline
\end{tabular}

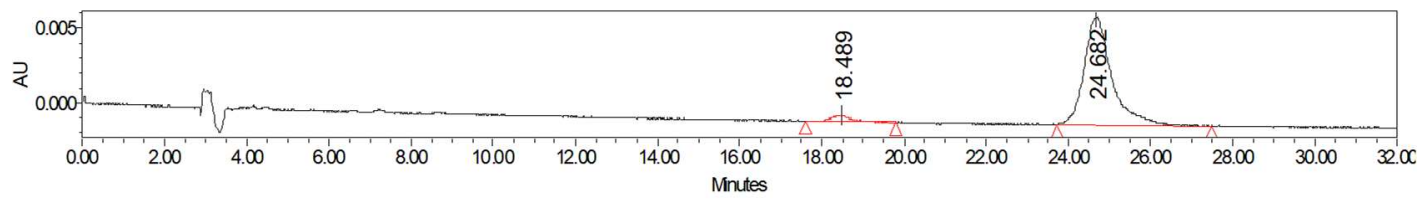

\begin{tabular}{|r|r|r|r|}
\hline & Retention Time & \multicolumn{1}{|c|}{ Area } & \% Area \\
\hline 1 & 18.489 & 14035 & 4.00 \\
\hline 2 & 24.682 & 336738 & 96.00 \\
\hline
\end{tabular}


<smiles>CN(C(=O)/C=C/c1ccccc1)c1ccc(-c2ccccc2)cc1Br</smiles>

$3 \mathbf{v}$

White solid. m.p. $65-70{ }^{\circ} \mathrm{C} .34 .4 \mathrm{mg}, 80 \%$ yield, $94 \%$ ee. Specific rotation $[\alpha]^{20}{ }_{\mathrm{D}}=+36.1\left(\mathrm{c}=0.37, \mathrm{CH}_{2} \mathrm{Cl}_{2}\right)$. Dissolved in $i-\mathrm{PrOH}$ for SFC; SFC (Chiralcel C1, $\mathrm{CO}_{2} / \mathrm{i}-\mathrm{PrOH}=80 / 20$, flow rate $\left.=1.0 \mathrm{~mL} / \mathrm{min}, 254 \mathrm{~nm}\right)$ : $\operatorname{tr}(\operatorname{minor})=13.07 \mathrm{~min}, \operatorname{tr}($ major $)=16.62 \mathrm{~min}$.

${ }^{1} \mathrm{H}$ NMR $\left(400 \mathrm{MHz}, \mathrm{CDCl}_{3}\right) \delta=7.92(\mathrm{~d}, \mathrm{~J}=2.2,1 \mathrm{H}), 7.85(\mathrm{~d}, \mathrm{~J}=15.5,1 \mathrm{H}), 7.68-7.61(\mathrm{~m}, 2 \mathrm{H}), 7.54-7.47(\mathrm{~m}, 3 \mathrm{H}), 7.45-7.38(\mathrm{~m}$, $1 \mathrm{H}), 7.37-7.30(\mathrm{~m}, 5 \mathrm{H}), 7.18(\mathrm{~d}, J=8.1,1 \mathrm{H}), 6.23(\mathrm{~d}, J=15.5,1 \mathrm{H}), 3.61(\mathrm{~s}, 3 \mathrm{H}), 1.54(\mathrm{~s}, 9 \mathrm{H})$.

${ }^{13} \mathrm{C}\left\{{ }^{1} \mathrm{H}\right\}$ NMR $\left(101 \mathrm{MHz}, \mathrm{CDCl}_{3}\right) \delta 167.2,149.6,146.4,143.0,140.0,134.0,132.2,132.1,130.9,129.2,128.9,128.4,128.3,128.0$, 127.2, 126.2, 117.6, 42.5, 36.6, 32.4.

HRMS (ESI+) $\mathrm{m} / \mathrm{z}$ calcd for $\mathrm{C}_{26} \mathrm{H}_{28} \mathrm{NO}_{3} \mathrm{~S}[\mathrm{M}+\mathrm{H}]^{+}: 434.1784$, found: 434.1779 .

IR (neat) 2963, 2361, 1680, 1618, 1482, 1353, 1233, 1153, 964, 916, 860, 761, 700, 545, 521.

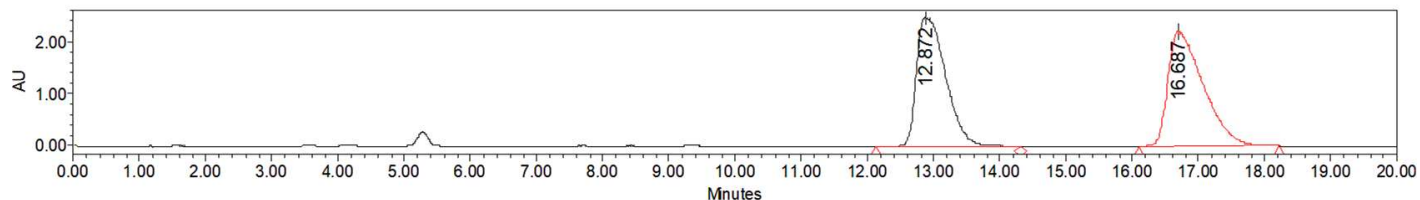

\begin{tabular}{|r|r|r|r|}
\hline & Retention Time & Area & \% Area \\
\hline 1 & 12.872 & 75035671 & 48.19 \\
\hline 2 & 16.687 & 80669321 & 51.81 \\
\hline
\end{tabular}

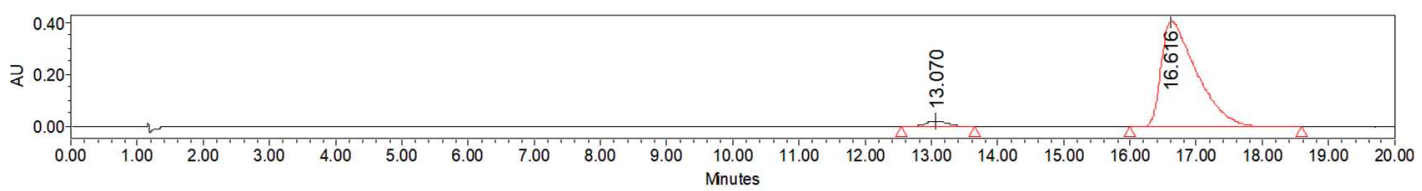

\begin{tabular}{|r|r|r|r|}
\hline & Retention Time & Area & \% Area \\
\hline 1 & 13.070 & 483564 & 3.12 \\
\hline 2 & 16.616 & 15007790 & 96.88 \\
\hline
\end{tabular}


<smiles>Cc1cc(C)c(N(C(=O)/C=C/c2ccccc2)C(C)(C)C)c(I)c1</smiles>

$3 \mathbf{w}$

White solid. m.p. $65-69^{\circ} \mathrm{C} .37 .2 \mathrm{mg}, 82 \%$ yield, $81 \%$ ee. Specific rotation $[\alpha]^{20}{ }_{\mathrm{D}}=+20.7\left(c=0.63, \mathrm{CH}_{2} \mathrm{Cl}_{2}\right)$. Dissolved in $i-\mathrm{PrOH}$ for HPLC; HPLC (Chiral IA column, $i$-PrOH $/ n$-hexane = 10/90, flow rate: $1.0 \mathrm{~mL} / \mathrm{min}, 254 \mathrm{~nm}$ ): $\operatorname{tr}(\operatorname{minor})=17.66 \mathrm{~min}, \operatorname{tr}(\mathrm{major})=25.49$ min.

${ }^{1} \mathrm{H}$ NMR $\left(400 \mathrm{MHz}, \mathrm{CDCl}_{3}\right) \delta=7.90(\mathrm{~d}, J=15.4,1 \mathrm{H}), 7.73-7.62(\mathrm{~m}, 1 \mathrm{H}), 7.42-7.29(\mathrm{~m}, 5 \mathrm{H}), 7.17(\mathrm{~d}, J=1.9,1 \mathrm{H}), 5.99(\mathrm{~d}, J=$ $15.5,1 \mathrm{H}), 3.79(\mathrm{~s}, 3 \mathrm{H}), 2.40(\mathrm{~d}, J=27.8,6 \mathrm{H})$.

${ }^{13} \mathrm{C}\left\{{ }^{1} \mathrm{H}\right\}$ NMR $\left(101 \mathrm{MHz}, \mathrm{CDCl}_{3}\right) \delta 165.9,147.3,142.0,141.1,138.6,134.5,134.0,132.5,130.8,128.9,128.4,116.1,102.0,44.4$, $20.7,20.5$

HRMS (ESI+) $\mathrm{m} / \mathrm{z}$ calcd for $\mathrm{C}_{18} \mathrm{H}_{18} \mathrm{INNaO}_{3} \mathrm{~S}[\mathrm{M}+\mathrm{Na}]^{+}: 477.9944$, found: 477.9943.

IR (neat) 2926, 2362, 1682, 1619, 1453, 1351, 1157, 966, 907, 857, 793, 762, 702, 565, 526.

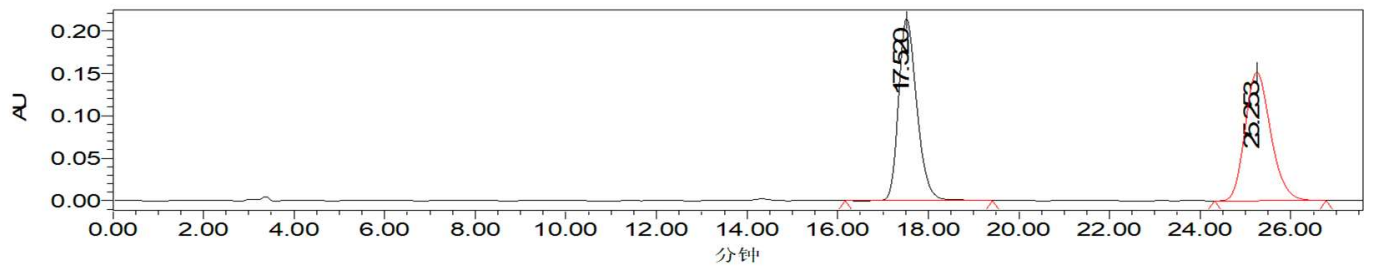

\begin{tabular}{|r|r|r|r|}
\hline & Retention Time & Area & \% Area \\
\hline 1 & 17.520 & 6015596 & 50.25 \\
\hline 2 & 25.253 & 5956469 & 49.75 \\
\hline
\end{tabular}

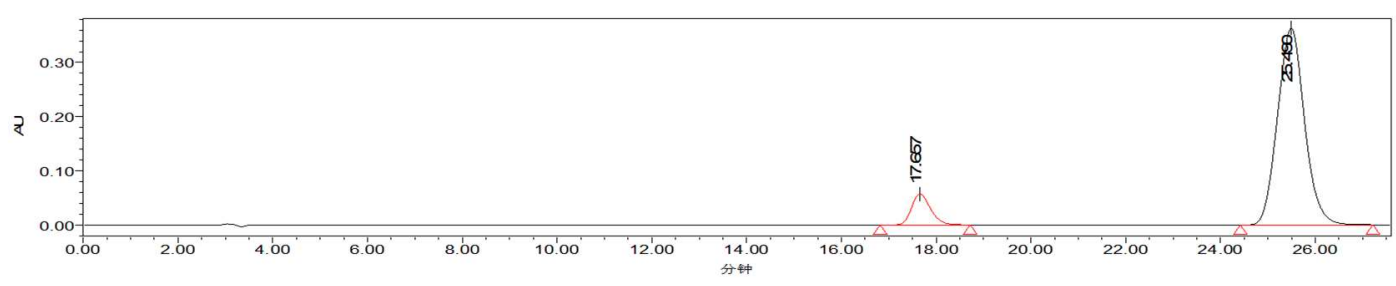

\begin{tabular}{|r|r|r|r|}
\hline & Retention Time & Area & \multicolumn{1}{c|}{$\%$ Area } \\
\hline 1 & 17.657 & 1572705 & 9.88 \\
\hline 2 & 25.490 & 14342437 & 90.12 \\
\hline
\end{tabular}


$N$-(4-Bromo-2-iodo-6-methylphenyl)- $N$-(methylsulfonyl)cinnamamide (3x)<smiles>CC(=O)N(C(=O)/C=C/c1ccccc1)c1c(C)cc(Br)cc1I</smiles>

White solid. m.p. $148-151^{\circ} \mathrm{C} .41 .9 \mathrm{mg}, 81 \%$ yield, $73 \%$ ee. Specific rotation $[\alpha]^{20}{ }_{\mathrm{D}}=+11.8\left(c=0.51, \mathrm{CH}_{2} \mathrm{Cl}_{2}\right)$. Dissolved in $i-\mathrm{PrOH}$ for HPLC; HPLC (Chiral IA column, $i$-PrOH $/ n$-hexane = 10/90, flow rate: $1.0 \mathrm{~mL} / \mathrm{min}, 254 \mathrm{~nm}$ ): $\operatorname{tr}(\operatorname{minor})=18.35 \mathrm{~min}, \operatorname{tr}(\mathrm{major})=25.88$ min.

${ }^{1} \mathrm{H}$ NMR $\left(400 \mathrm{MHz}, \mathrm{CDCl}_{3}\right) \delta=7.98(\mathrm{~d}, J=2.2,1 \mathrm{H}), 7.92(\mathrm{~d}, J=15.4,1 \mathrm{H}), 7.53(\mathrm{~d}, J=2.2,1 \mathrm{H}), 7.44-7.32(\mathrm{~m}, 5 \mathrm{H}), 5.96(\mathrm{~d}, J=$ $15.3,1 \mathrm{H}), 3.79(\mathrm{~s}, 3 \mathrm{H}), 2.46(\mathrm{~s}, 3 \mathrm{H})$.

${ }^{13} \mathrm{C}\left\{{ }^{1} \mathrm{H}\right\}$ NMR $\left(101 \mathrm{MHz}, \mathrm{CDCl}_{3}\right) \delta 165.4,148.0,143.1,140.2,136.6,134.6,133.8,131.1,129.0,128.5,124.7,115.4,102.9,44.6$, 20.6.

HRMS (ESI+) $\mathrm{m} / \mathrm{z}$ calcd for $\mathrm{C}_{17} \mathrm{H}_{15} \mathrm{BrINNaO}_{3} \mathrm{~S}[\mathrm{M}+\mathrm{Na}]^{+}: 541.8893$ and 543.8872 , found: 541.8884 and 843.8864 .

IR (neat) 2927, 2362, 1684, 1618, 1573, 1452, 1352, 1278, 1155, 966, 914, 867, 763, 699, 559, 529.

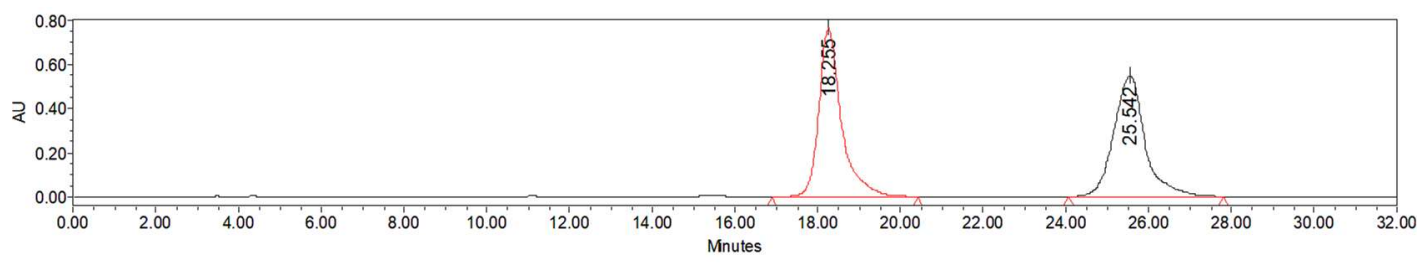

\begin{tabular}{|l|r|r|r|}
\hline & Retention Time & Area & \% Area \\
\hline 1 & 18.255 & 28618205 & 50.20 \\
\hline 2 & 25.542 & 28395503 & 49.80 \\
\hline
\end{tabular}

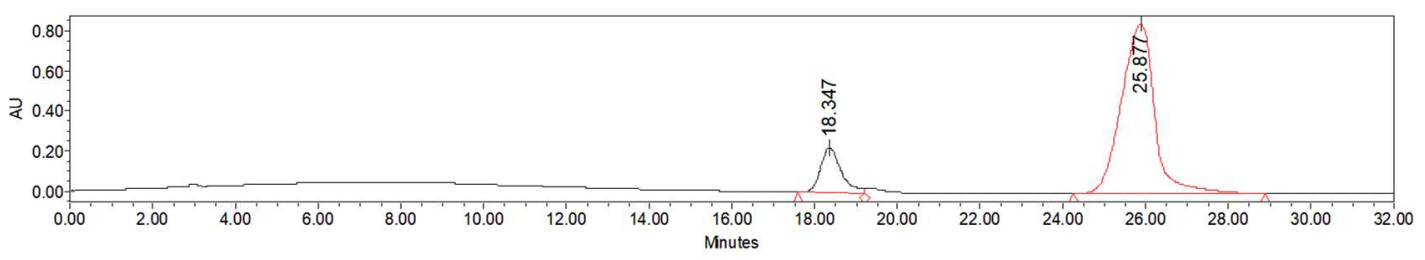

\begin{tabular}{|r|r|r|r|}
\hline & Retention Time & Area & \% Area \\
\hline 1 & 18.347 & 7502513 & 13.82 \\
\hline 2 & 25.877 & 46787527 & 86.18 \\
\hline
\end{tabular}


$N$-(2-Bromo-6-methylphenyl)- $N$-(methylsulfonyl)cinnamamide (3y)<smiles>CC(=O)N(C(=O)/C=C/c1ccccc1)c1c(C)cccc1Br</smiles>

$3 y$

White solid. m.p. $129-133{ }^{\circ} \mathrm{C}$. $33.4 \mathrm{mg}, 85 \%$ yield, $75 \%$ ee. Specific rotation $[\alpha]^{20}{ }_{\mathrm{D}}=-24.0\left(\mathrm{c}=0.29, \mathrm{CH}_{2} \mathrm{Cl}_{2}\right)$. Dissolved in $i-\mathrm{PrOH}$ for HPLC; HPLC (Chiral IA column, $i$-PrOH $/ n$-hexane = 10/90, flow rate: $1.0 \mathrm{~mL} / \mathrm{min}, 254 \mathrm{~nm}$ ): $\operatorname{tr}(\operatorname{minor})=17.14 \mathrm{~min}, \operatorname{tr}(\mathrm{major})=27.22$ $\min$.

${ }^{1} \mathrm{H}$ NMR $\left(400 \mathrm{MHz}, \mathrm{CDCl}_{3}\right) \delta=7.90(\mathrm{~d}, \mathrm{~J}=15.4,1 \mathrm{H}), 7.62-7.56(\mathrm{~m}, 1 \mathrm{H}), 7.40-7.26(\mathrm{~m}, 7 \mathrm{H}), 5.96(\mathrm{~d}, \mathrm{~J}=15.5,1 \mathrm{H}), 3.73(\mathrm{~s}, 3 \mathrm{H})$, $2.48(\mathrm{~s}, 3 \mathrm{H})$.

${ }^{13} \mathrm{C}\left\{{ }^{1} \mathrm{H}\right\}$ NMR $\left(101 \mathrm{MHz}, \mathrm{CDCl}_{3}\right) \delta 165.8,147.5,142.3,134.0,133.9,131.4,131.2,130.9,130.6,128.9,128.4,125.5,115.6,43.6$, 20.0 .

HRMS (ESI+) $\mathrm{m} / \mathrm{z}$ calcd for $\mathrm{C}_{17} \mathrm{H}_{16} \mathrm{BrNNaO}_{3} \mathrm{~S}[\mathrm{M}+\mathrm{Na}]^{+}: 415.9926$ and 417.9906 , found: 415.9935 and 417.9906 .

IR (neat) 2362, 1683, 1620, 1455, 1353, 1278, 1156, 966, 916, 848, 763, 702, 547, 522, 490.

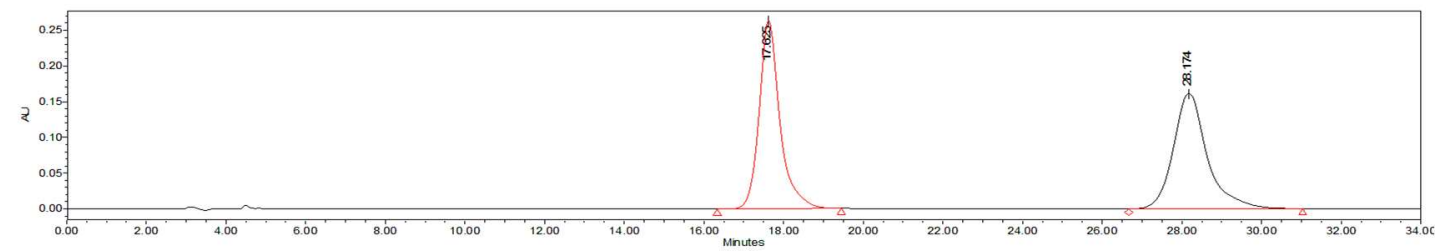

\begin{tabular}{|l|r|r|r|}
\hline & Retention Time & Area & \% Area \\
\hline 1 & 17.625 & 9512211 & 49.83 \\
\hline 2 & 28.174 & 9576721 & 50.17 \\
\hline
\end{tabular}

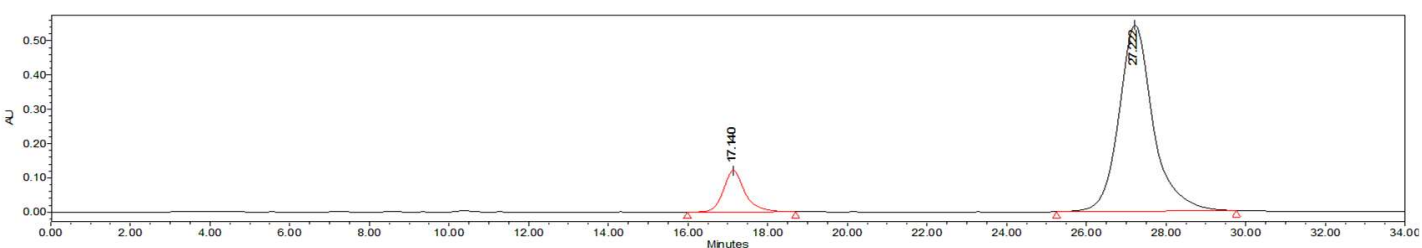

\begin{tabular}{|r|r|r|r|}
\hline & Retention Time & Area & \% Area \\
\hline 1 & 17.140 & 4569012 & 12.36 \\
\hline 2 & 27.222 & 32382540 & 87.64 \\
\hline
\end{tabular}


$N$-(2-(Tert-butyl)phenyl)- $N$-(phenylsulfonyl)cinnamamide (3aa)<smiles>O=C(/C=C/c1ccccc1)N(c1ccccc1Br)S(=O)(=O)c1ccccc1</smiles>

White solid. m.p. $80-85^{\circ} \mathrm{C}$. $28.1 \mathrm{mg}, 67 \%$ yield, $87 \%$ ee. Specific rotation $[\alpha]^{20}{ }_{\mathrm{D}}=+182.5\left(c=0.30, \mathrm{CH}_{2} \mathrm{Cl}_{2}\right)$. Dissolved in $i-\mathrm{PrOH}$ for HPLC; HPLC (Chiral ID column, $i$-PrOH $/ n$-hexane = 15/85, flow rate: $1.0 \mathrm{~mL} / \mathrm{min}, 254 \mathrm{~nm}$ ): $\operatorname{tr}($ major $)=16.87 \mathrm{~min}, \operatorname{tr}(\operatorname{minor})=19.46$ $\min$.

${ }^{1} \mathrm{H}$ NMR $\left(400 \mathrm{MHz}, \mathrm{CDCl}_{3}\right) \delta=8.16(\mathrm{dd}, J=7.8,1.6,2 \mathrm{H}), 7.75(\mathrm{dd}, J=8.2,1.5,1 \mathrm{H}), 7.72-7.64(\mathrm{~m}, 2 \mathrm{H}), 7.58(\mathrm{t}, J=7.7,2 \mathrm{H}), 7.53$ $-7.47(\mathrm{~m}, 1 \mathrm{H}), 7.33-7.21(\mathrm{~m}, 6 \mathrm{H}), 6.80(\mathrm{dd}, J=7.9,1.4,1 \mathrm{H}), 6.11(\mathrm{~d}, J=15.5,1 \mathrm{H}), 1.57(\mathrm{~s}, 9 \mathrm{H})$.

${ }^{13} \mathrm{C}\left\{{ }^{1} \mathrm{H}\right\}$ NMR $\left(101 \mathrm{MHz}, \mathrm{CDCl}_{3}\right) \delta 165.7,150.2,145.7,139.1,134.2,134.1,133.9,131.3,130.8,130.6,130.3,130.1,128.9,128.6$, 128.2, 127.2, 118.0, 36.7, 32.6.

HRMS (ESI+) $\mathrm{m} / \mathrm{z}$ calcd for $\mathrm{C}_{25} \mathrm{H}_{25} \mathrm{NNaO}_{3} \mathrm{~S}[\mathrm{M}+\mathrm{Na}]^{+}: 442.1447$, found: 442.1451 .

IR (neat) 2963, 2361, 1684, 1619, 1486, 1446, 1362, 1325, 1284, 1155, 1087, 984, 914, 856, 761, 736, 686, $593,566$.

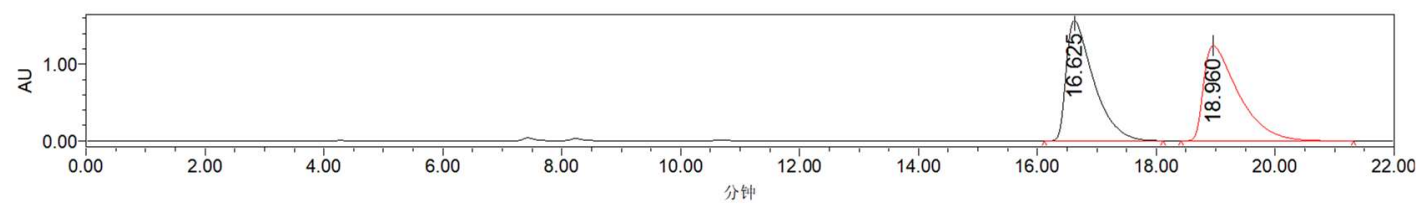

\begin{tabular}{|r|r|r|r|}
\hline & Retention Time & Area & \% Area \\
\hline 1 & 16.625 & 50273749 & 49.89 \\
\hline 2 & 18.960 & 50500994 & 50.11 \\
\hline
\end{tabular}

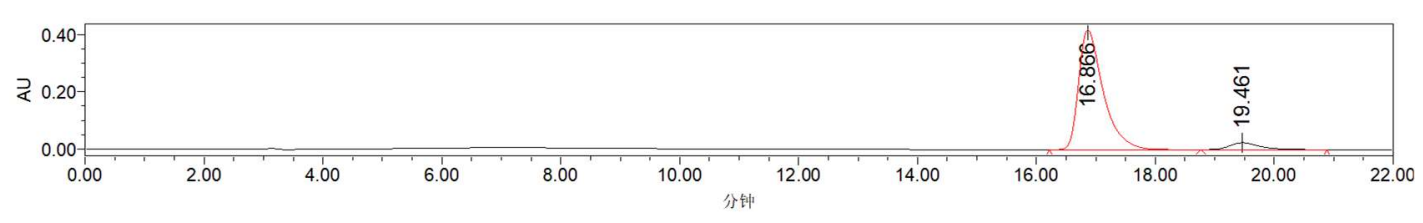

\begin{tabular}{|r|r|r|r|}
\hline & Retention Time & \multicolumn{1}{c|}{ Area } & \% Area \\
\hline 1 & 16.866 & 11938272 & 93.56 \\
\hline 2 & 19.461 & 821777 & 6.44 \\
\hline
\end{tabular}




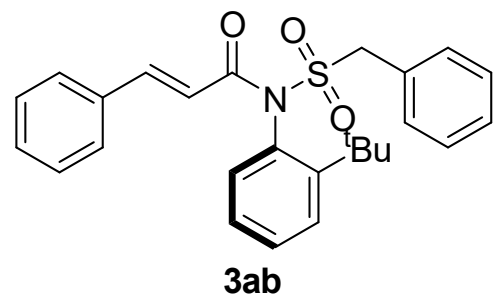

White solid. m.p. $58-63{ }^{\circ} \mathrm{C}$. $34.6 \mathrm{mg}, 80 \%$ yield, $94 \%$ ee. Specific rotation $[\alpha]^{20}{ }_{\mathrm{D}}=+232.8\left(\mathrm{c}=0.49, \mathrm{CH}_{2} \mathrm{Cl}_{2}\right)$. Dissolved in $i-\mathrm{PrOH}$ for HPLC; HPLC (Chiral ID column, $i$-PrOH $/ n$-hexane = 15/85, flow rate: $1.0 \mathrm{~mL} / \mathrm{min}, 254 \mathrm{~nm}$ ): $\operatorname{tr}(\operatorname{minor})=28.04 \mathrm{~min}$, $\operatorname{tr}($ major $)=32.87$ $\min$.

${ }^{1} \mathrm{H}$ NMR $\left(400 \mathrm{MHz}, \mathrm{CDCl}_{3}\right) \delta=7.85(\mathrm{~d}, J=15.5,1 \mathrm{H}), 7.59(\mathrm{dd}, J=8.2,1.5,1 \mathrm{H}), 7.51-7.37(\mathrm{~m}, 5 \mathrm{H}), 7.37-7.25(\mathrm{~m}, 6 \mathrm{H}), 6.89(\mathrm{td}$, $J=7.6,1.5,1 \mathrm{H}), 5.99(\mathrm{~d}, J=15.5,1 \mathrm{H}), 5.54(\mathrm{dd}, J=7.9,1.5,1 \mathrm{H}), 5.35(\mathrm{~d}, J=13.7,1 \mathrm{H}), 4.78(\mathrm{~d}, J=13.8,1 \mathrm{H})$.

${ }^{13} \mathrm{C}\left\{{ }^{1} \mathrm{H}\right\}$ NMR $\left(101 \mathrm{MHz}, \mathrm{CDCl}_{3}\right) \delta 167.0,150.0,146.0,134.1,133.1,132.1,131.5,130.8,130.0,129.7,129.4,129.1,128.9,128.3$, $127.9,127.1,117.5,60.2,36.3,32.2$.

HRMS (ESI+) $\mathrm{m} / \mathrm{z}$ calcd for $\mathrm{C}_{26} \mathrm{H}_{27} \mathrm{NNaO}_{3} \mathrm{~S}[\mathrm{M}+\mathrm{Na}]^{+}: 456.1624$, found: 456.1600 .

IR (neat) 2963, 2362, 1677, 1618, 1489, 1448, 1403, 1356, 1327, 1278, 1153, 987, 916.7, 859, 760, 698, 618, $568,538$.

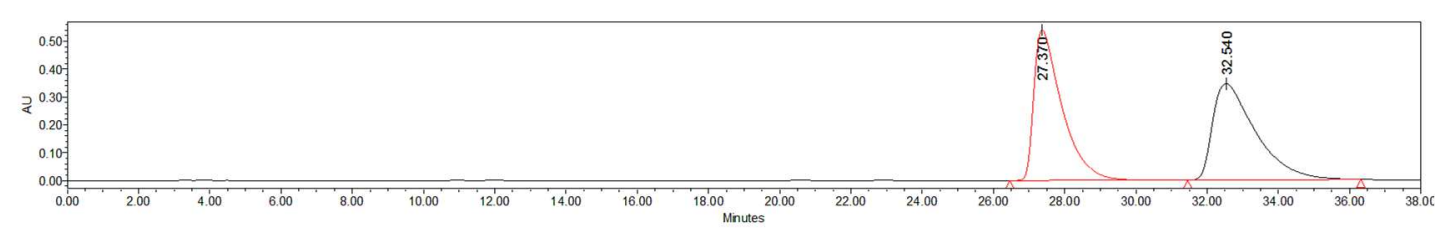

\begin{tabular}{|r|r|r|r|}
\hline & Retention Time & Area & \% Area \\
\hline 1 & 27.370 & 29519069 & 50.46 \\
\hline 2 & 32.540 & 28984655 & 49.54 \\
\hline
\end{tabular}

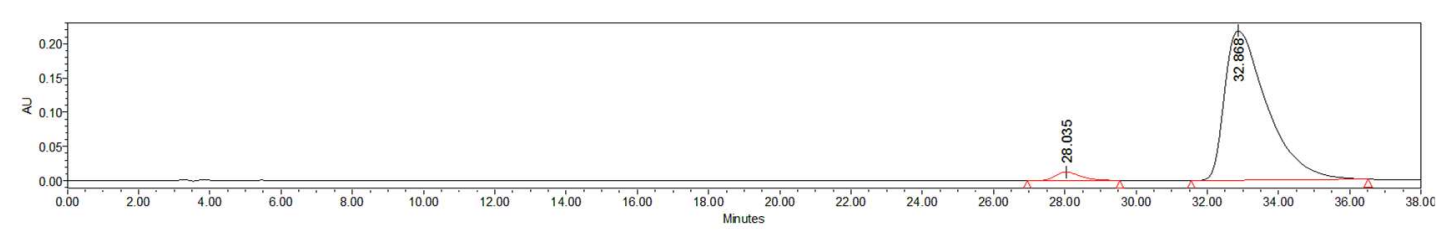

\begin{tabular}{|r|r|r|r|}
\hline & Retention Time & Area & \% Area \\
\hline 1 & 28.035 & 615283 & 3.30 \\
\hline 2 & 32.868 & 18054589 & 96.70 \\
\hline
\end{tabular}


<smiles>O=C(/C=C/c1ccccc1)N(c1ccccc1Br)S(=O)(=O)C1CCCCC1</smiles>

White solid. m.p. $73-76{ }^{\circ} \mathrm{C} .31 .0 \mathrm{mg}, 73 \%$ yield, $91 \%$ ee. Specific rotation $[\alpha]^{20}{ }_{\mathrm{D}}=+75.4\left(c=0.38, \mathrm{CH}_{2} \mathrm{Cl}_{2}\right)$. Dissolved in $i-\mathrm{PrOH}$ for HPLC; HPLC (Chiral ID column, $i$-PrOH $/ n$-hexane = 20/80, flow rate: $1.0 \mathrm{~mL} / \mathrm{min}, 254 \mathrm{~nm}$ ): $\operatorname{tr}(\operatorname{minor})=13.32 \mathrm{~min}, \operatorname{tr}(\operatorname{major})=14.70$ $\min$.

${ }^{1} \mathrm{H}$ NMR $\left(400 \mathrm{MHz}, \mathrm{CDCl}_{3}\right) \delta=7.79(\mathrm{~d}, J=15.5,1 \mathrm{H}), 7.70(\mathrm{dd}, J=8.2,1.5,1 \mathrm{H}), 7.50-7.42(\mathrm{~m}, 1 \mathrm{H}), 7.38-7.27(\mathrm{~m}, 6 \mathrm{H}), 7.15(\mathrm{dd}$, $J=7.9,1.5,1 \mathrm{H}), 6.20(\mathrm{~d}, J=15.6,1 \mathrm{H}), 4.23-4.09(\mathrm{~m}, 1 \mathrm{H}), 2.45(\mathrm{~d}, J=12.7,1 \mathrm{H}), 2.21(\mathrm{dd}, J=11.2,3.7,1 \mathrm{H}), 2.01-1.90(\mathrm{~m}, 2 \mathrm{H})$, $1.81-1.64(\mathrm{~m}, 3 \mathrm{H}), 1.49(\mathrm{~s}, 9 \mathrm{H}), 1.39$ (tdd, $J=12.9,7.1,3.5,2 \mathrm{H}), 1.30-1.22(\mathrm{~m}, 1 \mathrm{H})$.

${ }^{13} \mathrm{C}\left\{{ }^{1} \mathrm{H}\right\}$ NMR $\left(101 \mathrm{MHz}, \mathrm{CDCl}_{3}\right) \delta 166.7,150.6,145.9,134.1,133.3,132.7,130.7,130.3,130.1,128.9,128.3,127.2,117.7,64.1$, $36.6,32.2,27.3,26.0,25.4,25.2,25.0$.

HRMS (ESI+) $\mathrm{m} / \mathrm{z}$ calcd for $\mathrm{C}_{25} \mathrm{H}_{31} \mathrm{NNaO}_{3} \mathrm{~S}[\mathrm{M}+\mathrm{Na}]^{+}: 448.1917$, found: 448.1918 .

IR (neat) 2940, 2862, 2362, 1677, 1618, 1487, 1446, 1332, 1277, 1149, 1088, 987, 912, 855, 760, 684, 605, 552.

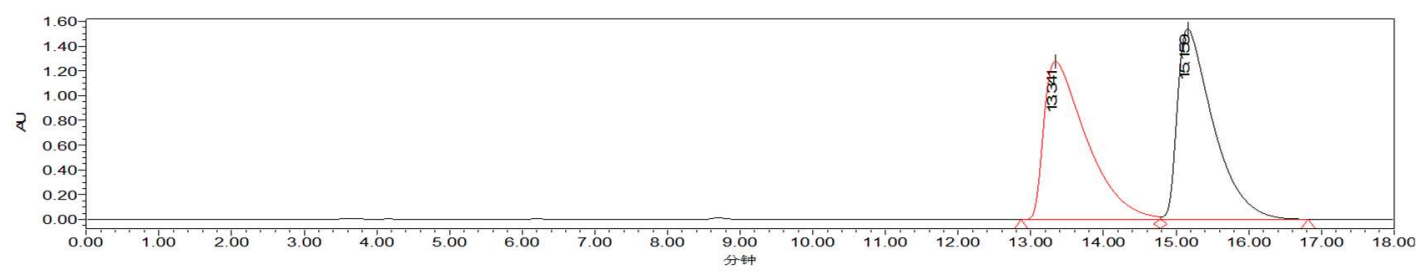

\begin{tabular}{|r|r|r|r|}
\hline & Retention Time & Area & \% Area \\
\hline 1 & 13.341 & 52134786 & 49.74 \\
\hline 2 & 15.159 & 52678949 & 50.26 \\
\hline
\end{tabular}

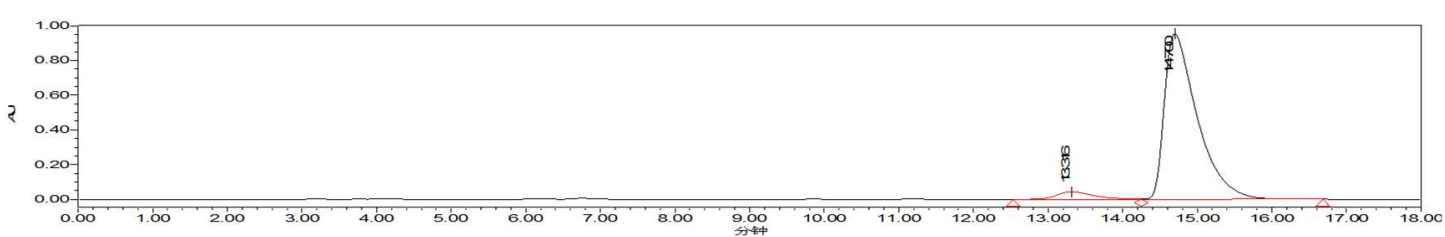

\begin{tabular}{|r|r|r|r|}
\hline & Retention Time & Area & \% Area \\
\hline 1 & 13.316 & 1439295 & 4.70 \\
\hline 2 & 14.700 & 29167618 & 95.30 \\
\hline
\end{tabular}


<smiles>CC(C)OC(=O)N([AsH-])c1ccccc1Br</smiles>

White solid. m.p. $141-146{ }^{\circ} \mathrm{C} .26 .8 \mathrm{mg}, 69 \%$ yield, $95 \%$ ee. Specific rotation $[\alpha]^{20}{ }_{\mathrm{D}}=+6.4\left(c=0.52, \mathrm{CH}_{2} \mathrm{Cl}_{2}\right)$. Dissolved in $i-\mathrm{PrOH}$ for HPLC; HPLC (Chiral ID column, $i-\mathrm{PrOH} / n$-hexane = 15/85, flow rate: $1.0 \mathrm{~mL} / \mathrm{min}, 254 \mathrm{~nm})$ : $\operatorname{tr}(\operatorname{minor})=9.98 \mathrm{~min}, \operatorname{tr}($ major $)=11.71$ $\min$.

${ }^{1} \mathrm{H}$ NMR $\left(400 \mathrm{MHz}, \mathrm{CDCl}_{3}\right) \delta=7.57$ (dd, $\left.J=8.2,1.6,1 \mathrm{H}\right), 7.36$ (ddd, $\left.J=8.2,7.2,1.5,1 \mathrm{H}\right), 7.22$ (ddd, $\left.J=7.9,7.2,1.6,1 \mathrm{H}\right), 7.05(\mathrm{dd}$, $J=7.8,1.5,1 \mathrm{H}$ ), 5.06 (hept, $J=6.3,1 \mathrm{H}), 3.51(\mathrm{~s}, 3 \mathrm{H}), 1.43(\mathrm{~s}, 9 \mathrm{H}), 1.21$ (dd, $J=20.1,6.3,6 \mathrm{H})$.

${ }^{13} \mathrm{C}\left\{{ }^{1} \mathrm{H}\right\}$ NMR $\left(101 \mathrm{MHz}, \mathrm{CDCl}_{3}\right) \delta 153.2,148.7,133.3,130.8,129.6,129.5,126.9,72.2,41.9,36.0,31.9,21.7$.

HRMS (ESI+) $\mathrm{m} / \mathrm{z}$ calcd for $\mathrm{C}_{21} \mathrm{H}_{27} \mathrm{NNaO}_{4} \mathrm{~S}[\mathrm{M}+\mathrm{Na}]^{+}: 412.1553$, found: 412.1554 .

IR (neat) 2923, 2854, 1730, 1597, 1489, 1465, 1439, 1365, 1275, 1217, 1166, 1106, 1086, 1061, 970, 902, 817, 791, 766, 704 $668,646,583,545,414$.

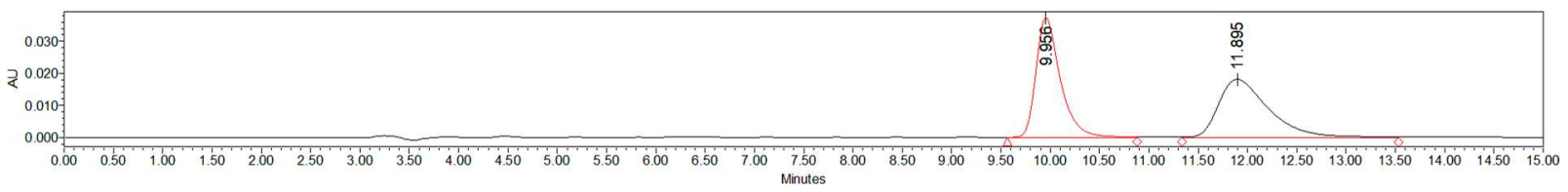

\begin{tabular}{|r|r|r|r|}
\hline & Retention Time & Area & \% Area \\
\hline 1 & 9.956 & 640951 & 49.99 \\
\hline 2 & 11.895 & 641217 & 50.01 \\
\hline
\end{tabular}

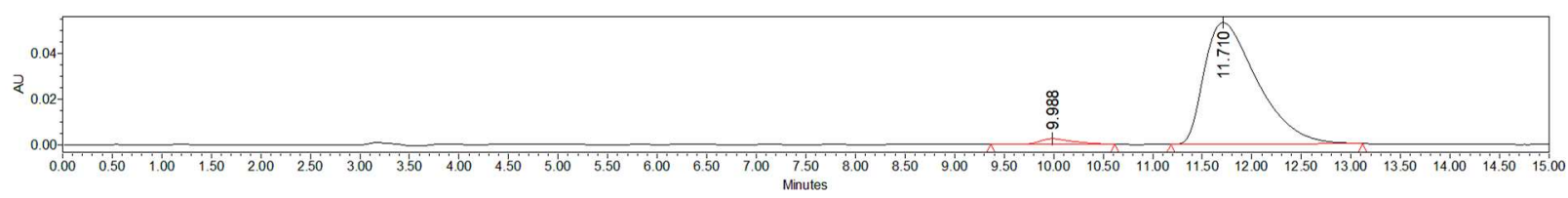

\begin{tabular}{|r|r|r|r|}
\hline & Retention Time & Area & \% Area \\
\hline 1 & 9.988 & 55173 & 2.71 \\
\hline 2 & 11.710 & 1977775 & 97.29 \\
\hline
\end{tabular}


<smiles>CC(C)OC(=O)N(C)S(C)(=O)=O</smiles>

Colorless oil. $23.8 \mathrm{mg}, 76 \%$ yield, $96 \%$ ee. Specific rotation $[\alpha]^{20}{ }_{\mathrm{D}}=+27.4\left(c=0.40, \mathrm{CH}_{2} \mathrm{Cl}_{2}\right)$. Dissolved in $i$-PrOH for HPLC; HPLC (Chiral IC column, $i-\mathrm{PrOH} / n$-hexane $=10 / 90$, flow rate: $1.0 \mathrm{~mL} / \mathrm{min}, 254 \mathrm{~nm}$ ): $\operatorname{tr}($ major $)=26.25 \mathrm{~min}, \operatorname{tr}(\operatorname{minor})=34.31 \mathrm{~min}$.

${ }^{1} \mathrm{H}$ NMR $\left(400 \mathrm{MHz}, \mathrm{CDCl}_{3}\right) \delta=7.57$ (dd, $\left.J=8.2,1.6,1 \mathrm{H}\right), 7.36$ (ddd, $\left.J=8.2,7.2,1.5,1 \mathrm{H}\right), 7.22$ (ddd, $J=7.9,7.2,1.6,1 \mathrm{H}$ ), 7.05 (dd, $J=7.8,1.5,1 \mathrm{H}), 5.06$ (hept, $J=6.3,1 \mathrm{H}), 3.51(\mathrm{~s}, 3 \mathrm{H}), 1.43(\mathrm{~s}, 9 \mathrm{H}), 1.21(\mathrm{dd}, J=20.1,6.3,6 \mathrm{H})$.

${ }^{13} \mathrm{C}\left\{{ }^{1} \mathrm{H}\right\}$ NMR $\left(101 \mathrm{MHz}, \mathrm{CDCl}_{3}\right) \delta 153.2,148.7,133.3,130.8,129.6,129.5,126.9,72.2,41.9,36.0,31.9,21.7$.

HRMS (ESI+) $\mathrm{m} / \mathrm{z}$ calcd for $\mathrm{C}_{15} \mathrm{H}_{24} \mathrm{NO}_{4} \mathrm{~S}[\mathrm{M}+\mathrm{H}]^{+}: 314.1421$, found: 314.1417

IR (neat) 2970, 1723, 1488, 1439, 1354, 1320, 1263, 1224, 1160, 1101, 1054, 963, 890, 867, 825, 773, 759, 649, 575, 544, 526, $500,421$.

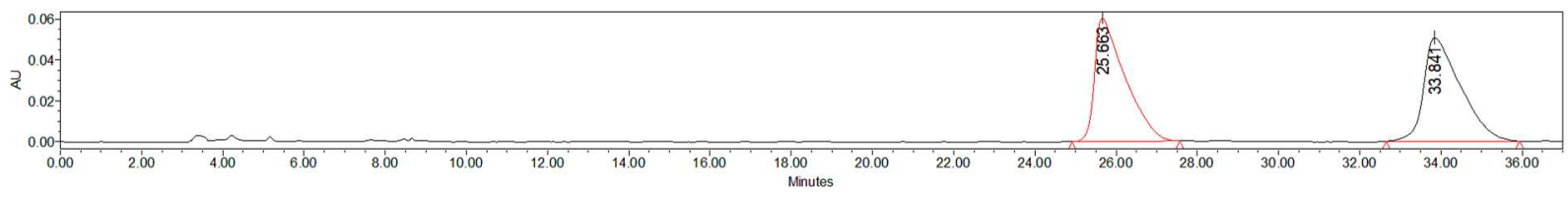

\begin{tabular}{|r|r|r|r|}
\hline & Retention Time & Area & \% Area \\
\hline 1 & 25.663 & 3134693 & 49.73 \\
\hline 2 & 33.841 & 3169047 & 50.27 \\
\hline
\end{tabular}

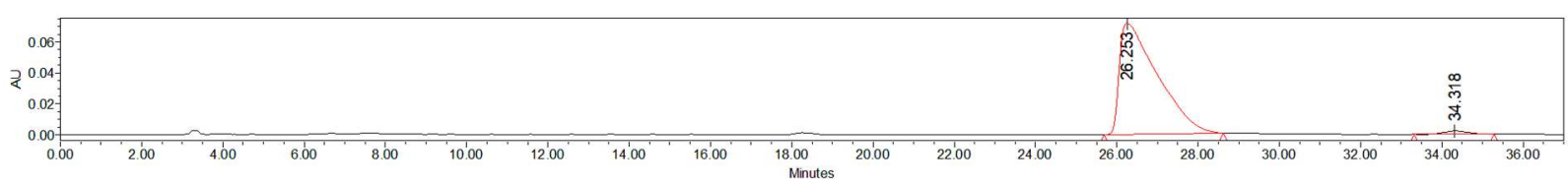

\begin{tabular}{|r|r|r|r|}
\hline & Retention Time & \multicolumn{1}{c|}{ Area } & \% Area \\
\hline 1 & 26.253 & 4673602 & 98.21 \\
\hline 2 & 34.318 & 85237 & 1.79 \\
\hline
\end{tabular}


$N-(2-($ Tert-butyl)phenyl)- $N$-(methylsulfonyl)acetamide (4c)<smiles>CC(=O)N(c1ccccc1)c1ccc(C)cc1Br</smiles>

White solid. m.p. $102-106{ }^{\circ} \mathrm{C}$. $11.9 \mathrm{mg}, 44 \%$ yield, $90 \%$ ee. Specific rotation $[\alpha]^{20}{ }_{\mathrm{D}}=+43.5\left(c=0.20, \mathrm{CH}_{2} \mathrm{Cl}_{2}\right)$. Dissolved in $i-\mathrm{PrOH}$ for HPLC; HPLC (Chiral ID column, $i$-PrOH $/ n$-hexane = 15/85, flow rate: $1.0 \mathrm{~mL} / \mathrm{min}, 254 \mathrm{~nm}$ ): $\operatorname{tr}(\operatorname{minor})=18.76 \mathrm{~min}, \operatorname{tr}(\operatorname{major})=20.24$ $\min$.

${ }^{1} \mathrm{H}$ NMR $\left(400 \mathrm{MHz}, \mathrm{CDCl}_{3}\right) \delta=7.65(\mathrm{dd}, J=8.2,1.6,1 \mathrm{H}), 7.48-7.40(\mathrm{~m}, 1 \mathrm{H}), 7.29(\mathrm{dd}, J=7.8,1.6,1 \mathrm{H}), 7.07(\mathrm{dd}, J=7.8,1.5,1 \mathrm{H})$, $3.50(\mathrm{~s}, 3 \mathrm{H}), 1.97(\mathrm{~s}, 3 \mathrm{H}), 1.47(\mathrm{~s}, 9 \mathrm{H})$.

${ }^{13} \mathrm{C}\left\{{ }^{1} \mathrm{H}\right\}$ NMR $\left(101 \mathrm{MHz}, \mathrm{CDCl}_{3}\right) \delta 172.6,148.7,134.0,131.3,130.6,130.4,127.5,42.2,36.4,32.2,25.5$.

HRMS (ESI+) $\mathrm{m} / \mathrm{z}$ calcd for $\mathrm{C}_{13} \mathrm{H}_{19} \mathrm{NNaO}_{3} \mathrm{~S}[\mathrm{M}+\mathrm{Na}]^{+}: 292.0978$, found: 292.0977 .

IR (neat) 2922, 1698, 1487, 1437, 1348, 1247, 1215, 1158, 1215, 1158, 1091, 1054, 1014, 961, 892, 862, 837, 766, 715, 610, 540, 521, 499.

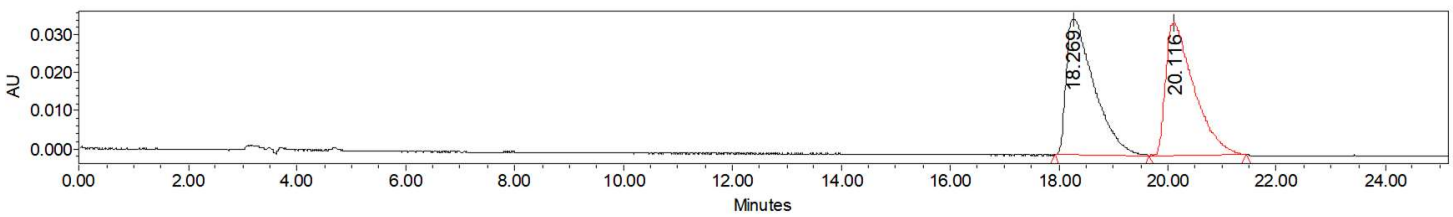

\begin{tabular}{|r|r|r|r|}
\hline & Retention Time & Area & \% Area \\
\hline 1 & 18.269 & 1272606 & 50.18 \\
\hline 2 & 20.116 & 1263447 & 49.82 \\
\hline
\end{tabular}

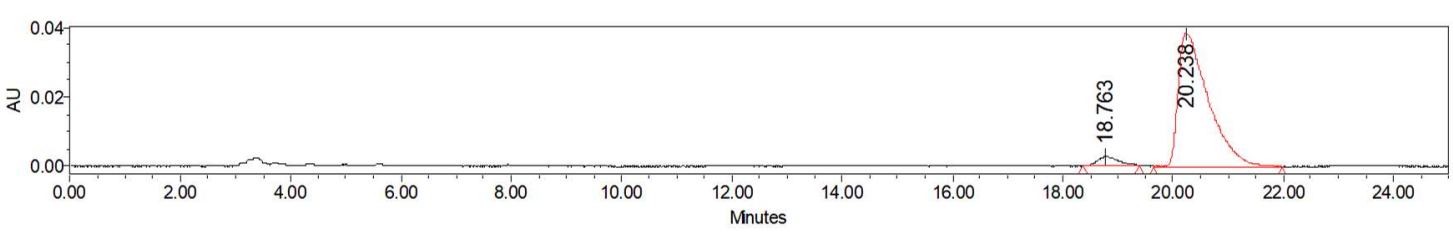

\begin{tabular}{|r|r|r|r|}
\hline & Retention Time & Area & \% Area \\
\hline 1 & 18.763 & 73722 & 4.82 \\
\hline 2 & 20.238 & 1457198 & 95.18 \\
\hline
\end{tabular}




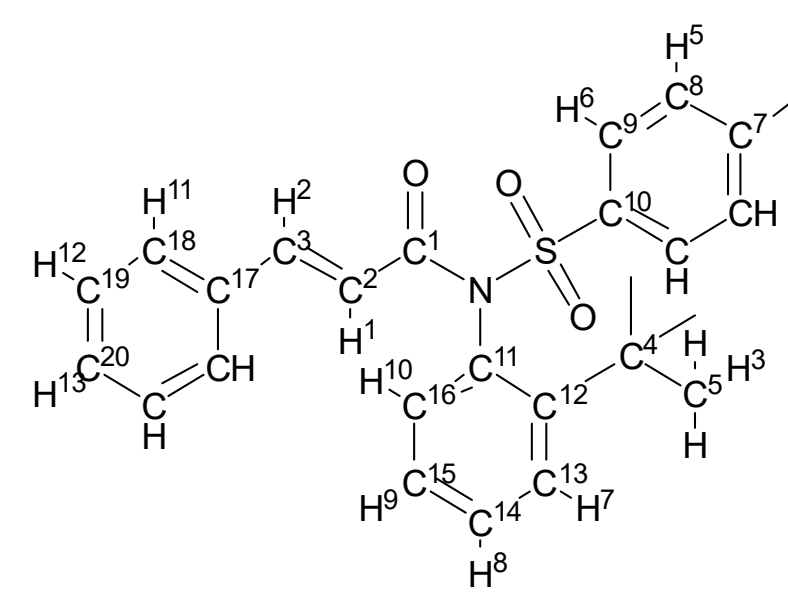

3a
$\mathrm{H}^{1} \delta$ H. 7

$\mathrm{H}^{2} \delta 7.68$

$\mathrm{H}^{3} \delta 3$.

$3^{4} \delta 2.46$

$H^{5} \delta 528$.

$\mathrm{H}^{6} \delta 8.04$

$\mathrm{H}^{7}$ ??7.74

$\mathrm{H}^{8} \delta 7.48$

$H^{9} \delta 7.32-7.29$

$\mathrm{H}^{10} \delta 6.80$

$\mathrm{H}^{11} \delta 7.23-7.20$

$\mathrm{H}^{12} \delta 7.27-2.27$

$\mathrm{H}^{13} \delta 7.29-7.28$
$C^{1} ? 165.7 \quad C^{11} \delta 127.1$

$C^{2} \delta 2.272 \quad C^{12} \delta 150.2$

$2^{3} \delta 145.5 \quad C^{13} \delta 130.6$

$C^{4}$ ?36.7 $\quad C^{14} ? 130.2$

$C^{5} \delta 32.6 \quad C^{15} \delta 134.2$

$C^{6} \delta 21.7 \quad C^{16} \delta 131.3$

$C^{7} \delta 145.0 \quad C^{17} \delta 134.3$

$C^{8} \delta 129.3 \quad C^{18} \delta$ Xl 5.5

$C^{9} \delta \quad 272 \quad \delta^{19} \delta 128.8$

$\mathrm{C}^{10} \delta \delta .7 \quad \mathrm{C}^{20} \delta 130.7$

COSY Spectra of $3 a$

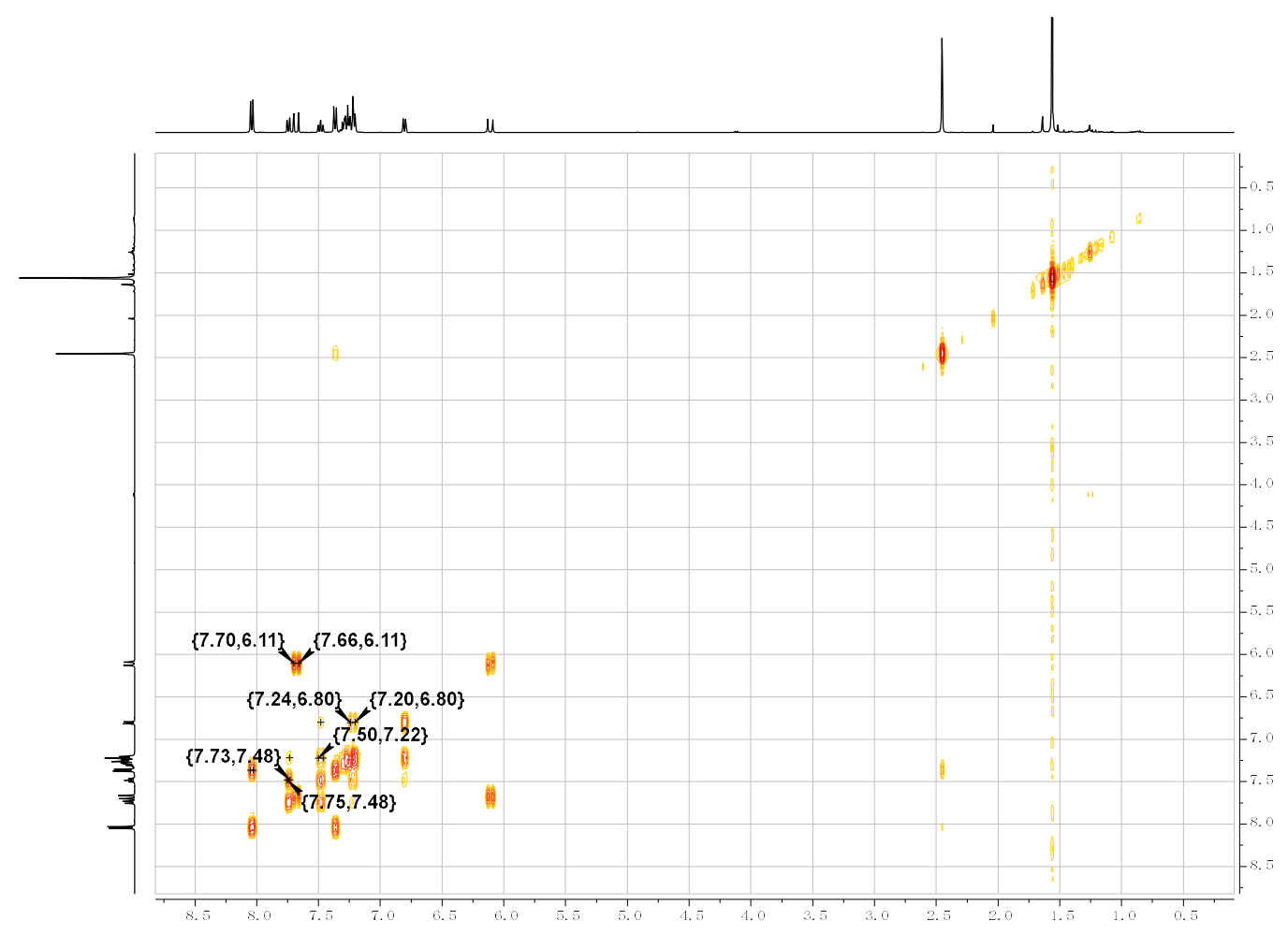




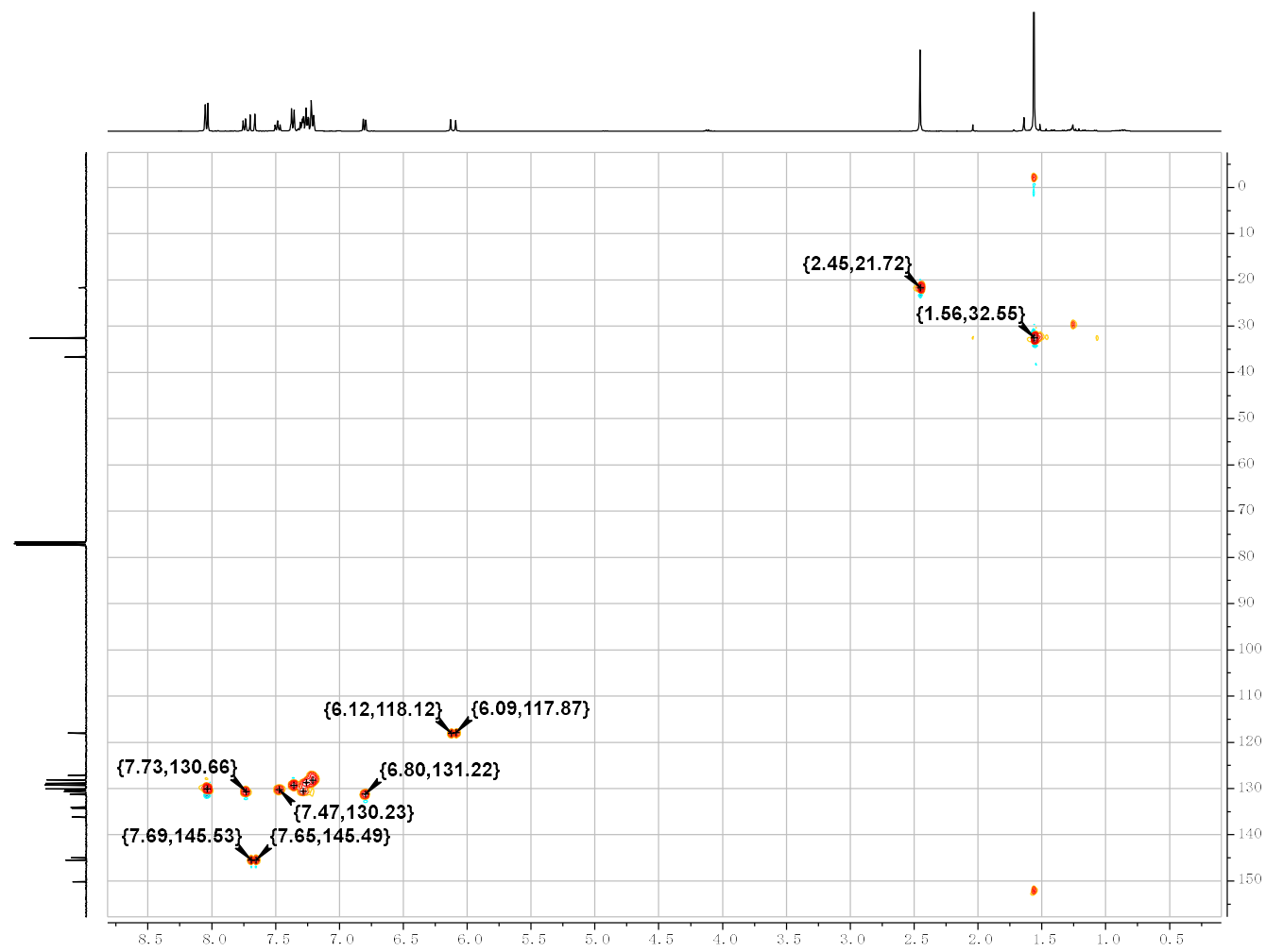

HMBC Spectra of $3 a$

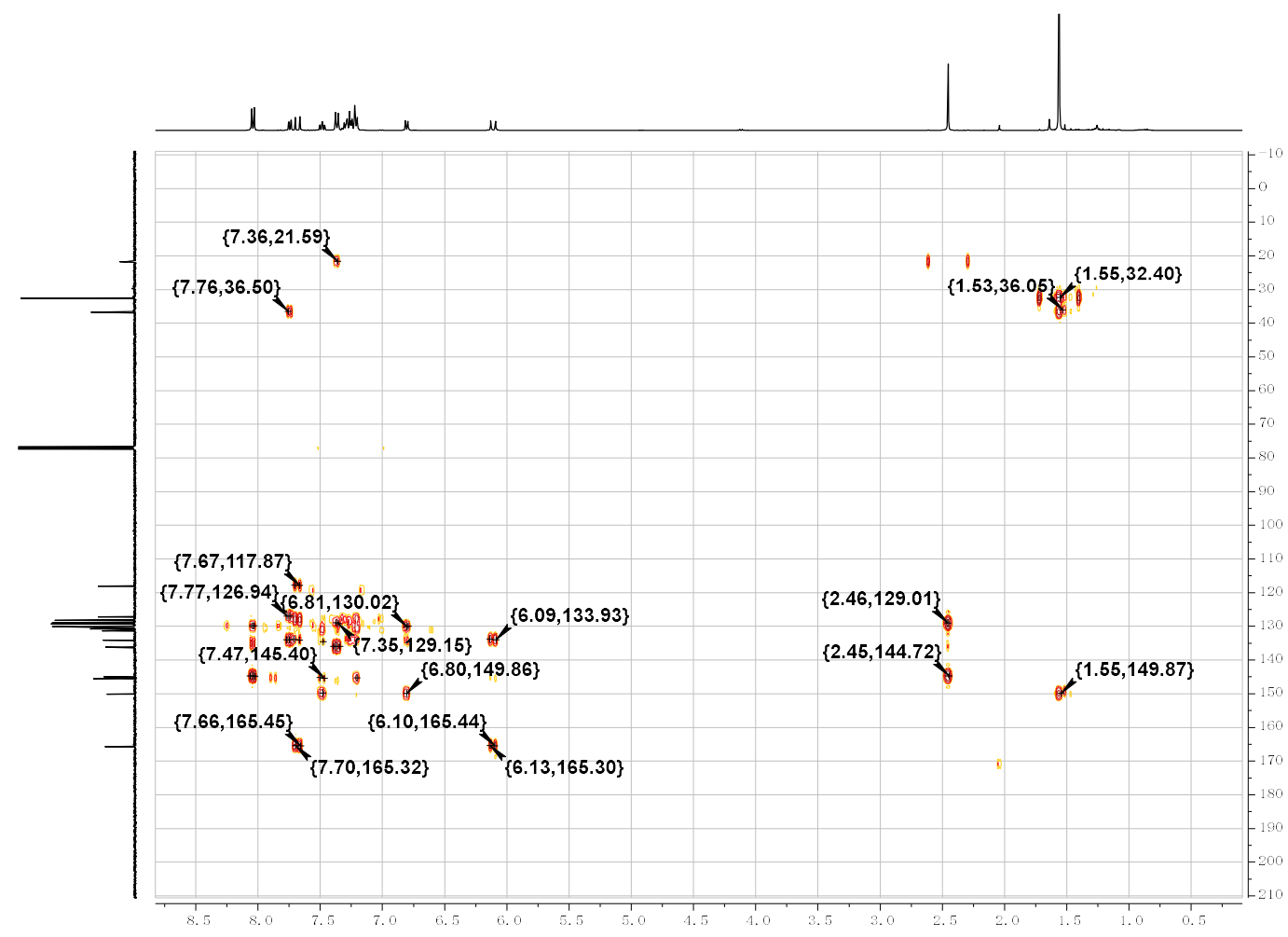




\section{The X-ray data for 31}

The colourless crystals in block shape were picked up and mounted for the single-crystal X-ray diffraction. The measurements were taken in a Bruker D8 Venture diffractometer equipped with Photon II CMOS detector by omega and phi scan methods. The data were integrated by $A P E X 3$ supplied by Bruker Inc with multi-scan absorption corrections. The structure solution and refinement were processed by SHELXTL (version 6.14) and OLEX 2.3 program package $\mathrm{e}^{4,5,6,7}$.

The crystal suitable for X-ray structure analysis was obtained from the solution of $3 \mathrm{I}$ in $\mathrm{CH}_{2} \mathrm{Cl}_{2}$ and $n$-hexane. The data has been deposited at the Cambridge Crystallographic Data Center (CCDC 1999875). The flack parameter refined by Parsons' method is $0.013(17)$, which indicates the absolute structure of this crystal was correctly assigned.

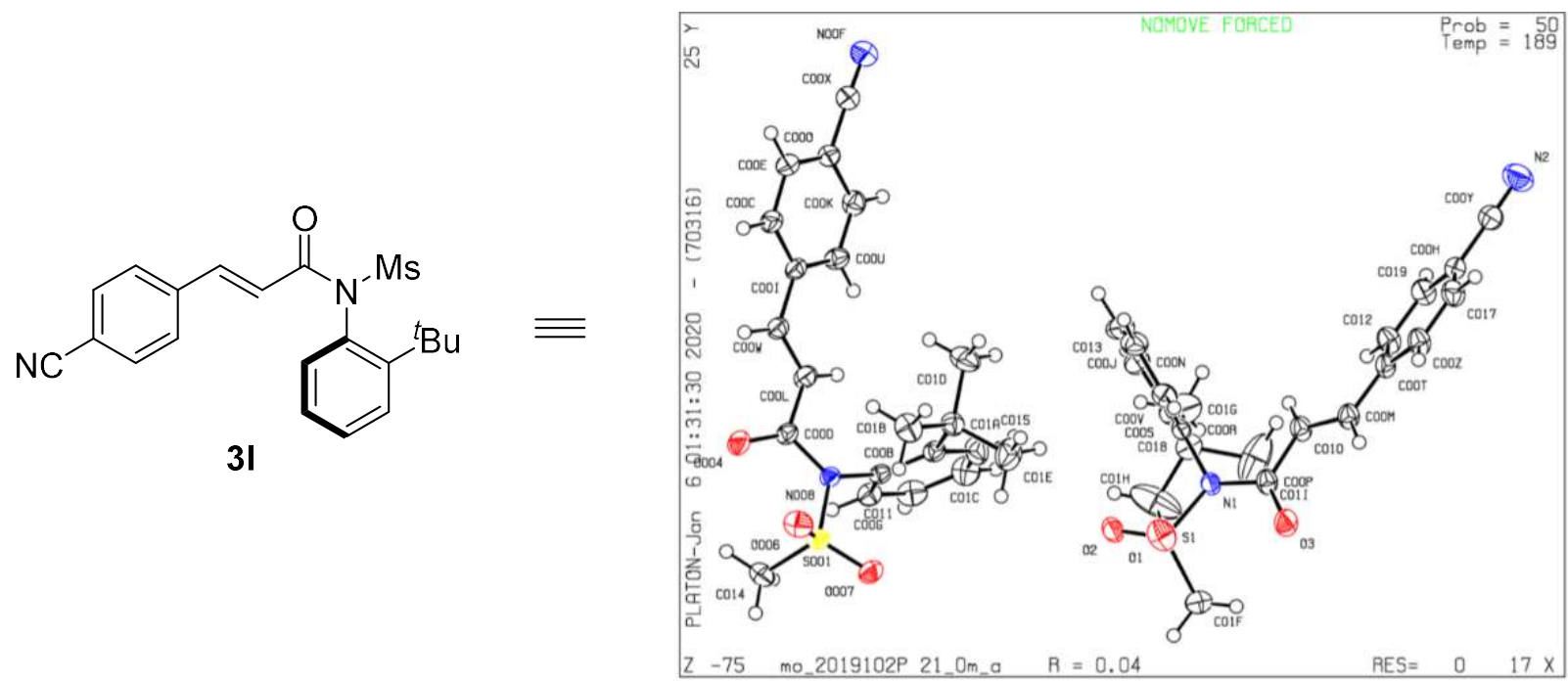

Figure 1. The thermal ellipsoid figure of $3 \mathrm{I}$ with $50 \%$ probablities

Crystallographic Data for $\mathrm{C} 21 \mathrm{H} 22 \mathrm{~N} 2 \mathrm{O} 3 \mathrm{~S}$.

\begin{tabular}{|c|c|}
\hline Formula & $\mathrm{C} 21 \mathrm{H} 22 \mathrm{~N} 2 \mathrm{O} 3 \mathrm{~S}$ \\
\hline Formula mass (amu) & 382.46 \\
\hline Space group & P 21 \\
\hline$a(\AA)$ & $7.5103(4)$ \\
\hline$b(\AA)$ & $33.3848(16)$ \\
\hline$c(\AA)$ & $8.5353(4)$ \\
\hline$\alpha(\mathrm{deg})$ & 90 \\
\hline$\beta(\mathrm{deg})$ & $114.131(2)$ \\
\hline$Y(\operatorname{deg})$ & 90 \\
\hline$V\left(\AA^{3}\right)$ & 1953.04(17) \\
\hline$Z$ & 4 \\
\hline$\lambda(\AA)$ & 0.71073 \\
\hline$T(\mathrm{~K})$ & $189 \mathrm{~K}$ \\
\hline$\rho_{\text {calcd }}\left(\mathrm{g} \mathrm{cm}^{-3}\right)$ & 1.301 \\
\hline$\mu\left(\mathrm{mm}^{-1}\right)$ & 0.189 \\
\hline Transmission factors & $0.693-0.746$ \\
\hline $2 \theta_{\max }(\mathrm{deg})$ & 28.318 \\
\hline No. of unique data, including $F_{0}^{2}<0$ & 9630 \\
\hline No. of unique data, with $F_{0}^{2}>2 \sigma\left(F_{0}^{2}\right)$ & 9038 \\
\hline No. of variables & 495 \\
\hline$R(F)$ for $F_{0}^{2}>2 \sigma\left(F_{0}^{2}\right)$ & 0.0355 \\
\hline$R_{\mathrm{w}}\left(F_{\mathrm{o}}^{2}\right)$ & 0.0906 \\
\hline Goodness of fit & 1.042 \\
\hline
\end{tabular}


13. Copy of ${ }^{1} \mathrm{H},{ }^{13} \mathrm{C}\left\{{ }^{1} \mathrm{H}\right\}$ and ${ }^{19} \mathrm{~F}\left\{{ }^{1} \mathrm{H}\right\}$ NMR Spectra.

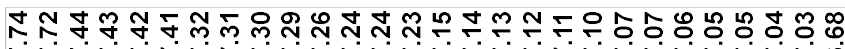

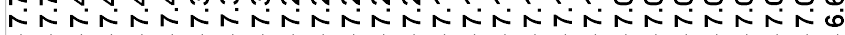

$\stackrel{m}{i} \quad \stackrel{m}{i}$
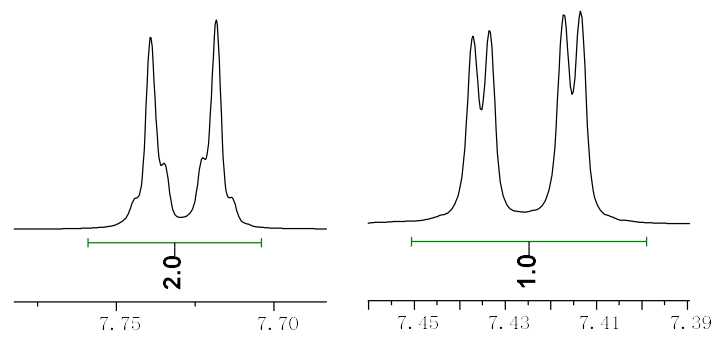

$400 \mathrm{MHz}, \mathrm{CDCl}_{3}-{ }^{1} \mathrm{H} \mathrm{NMR}$<smiles>Cc1ccccc1</smiles><smiles>CNS(C)(=O)=O</smiles>
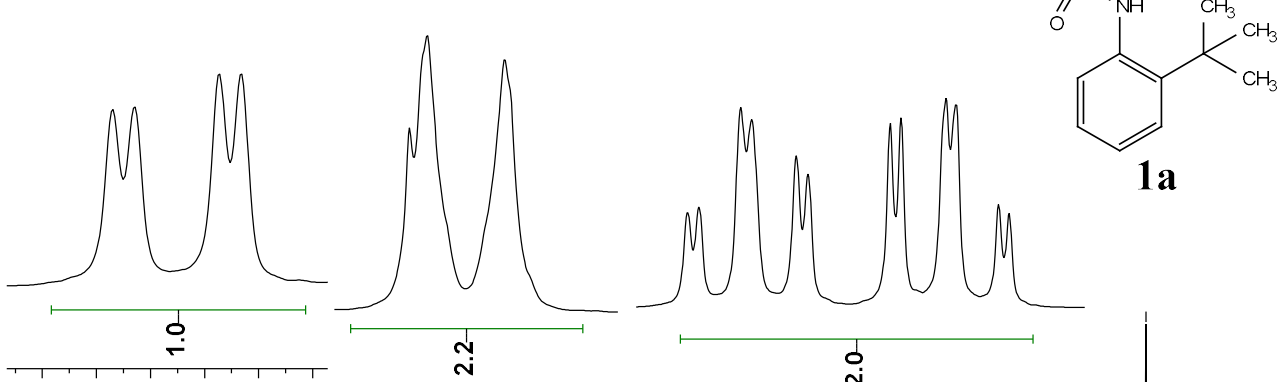

$1 \mathrm{a}$

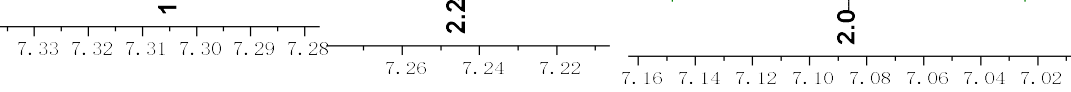

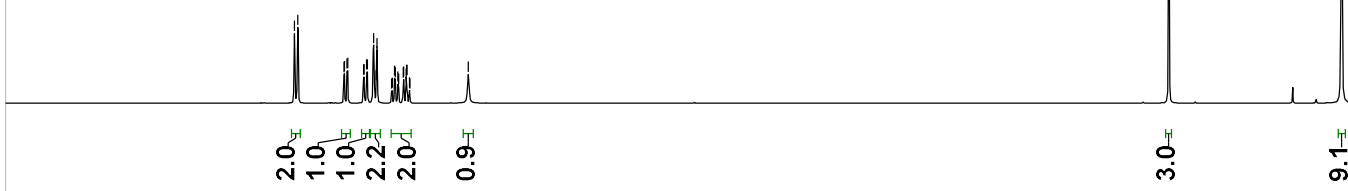

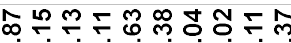

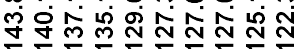

लै

$101 \mathrm{MHz}, \mathrm{CDCl}_{3}{ }^{-13} \mathrm{C} \mathrm{NMR}$

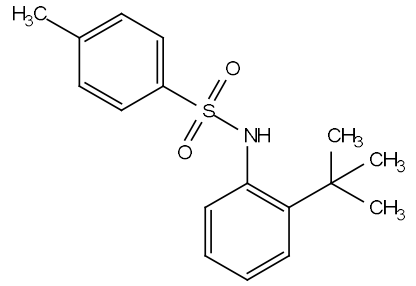

$1 \mathrm{a}$

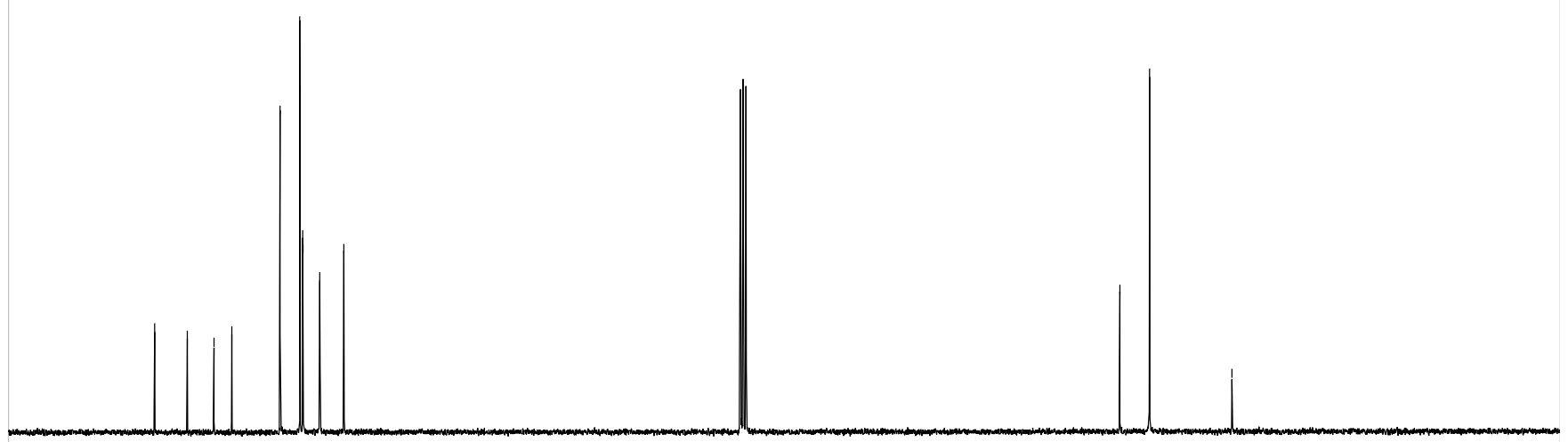




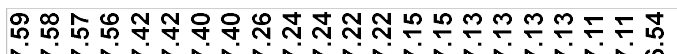
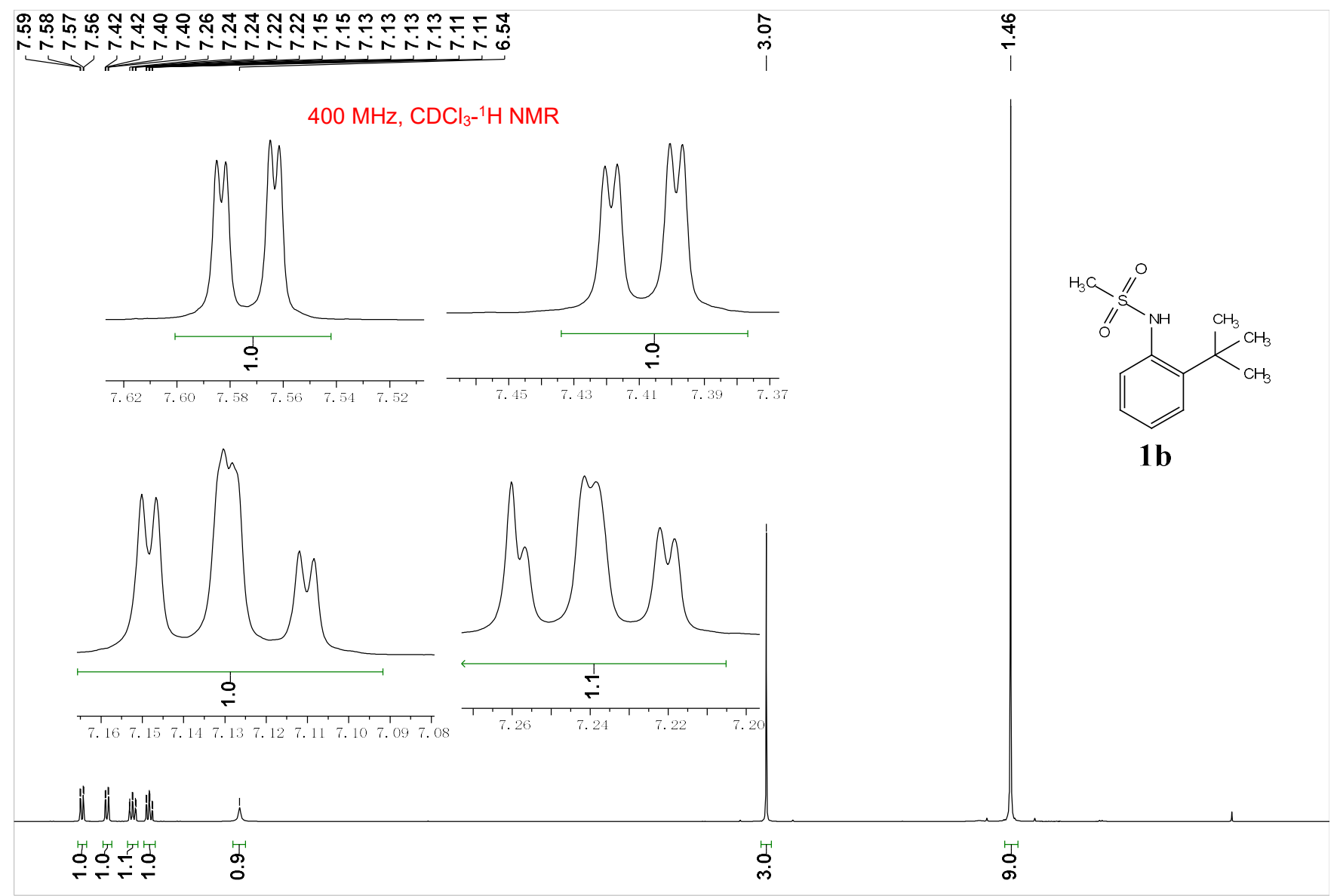

$\stackrel{1}{1}$



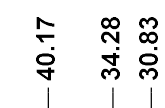

$101 \mathrm{MHz}, \mathrm{CDCl}_{3}{ }^{-13} \mathrm{C}$ NMR

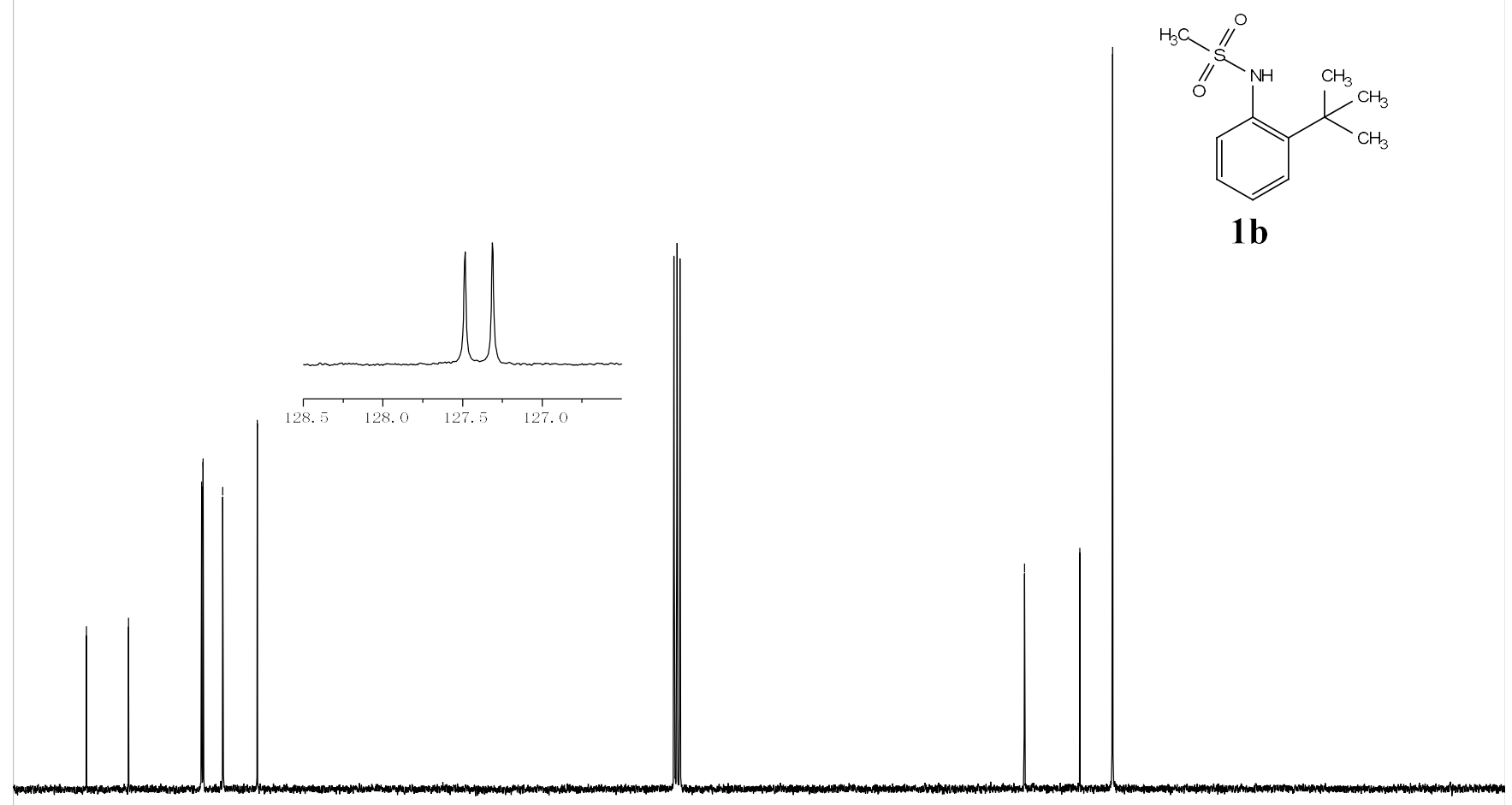

54 


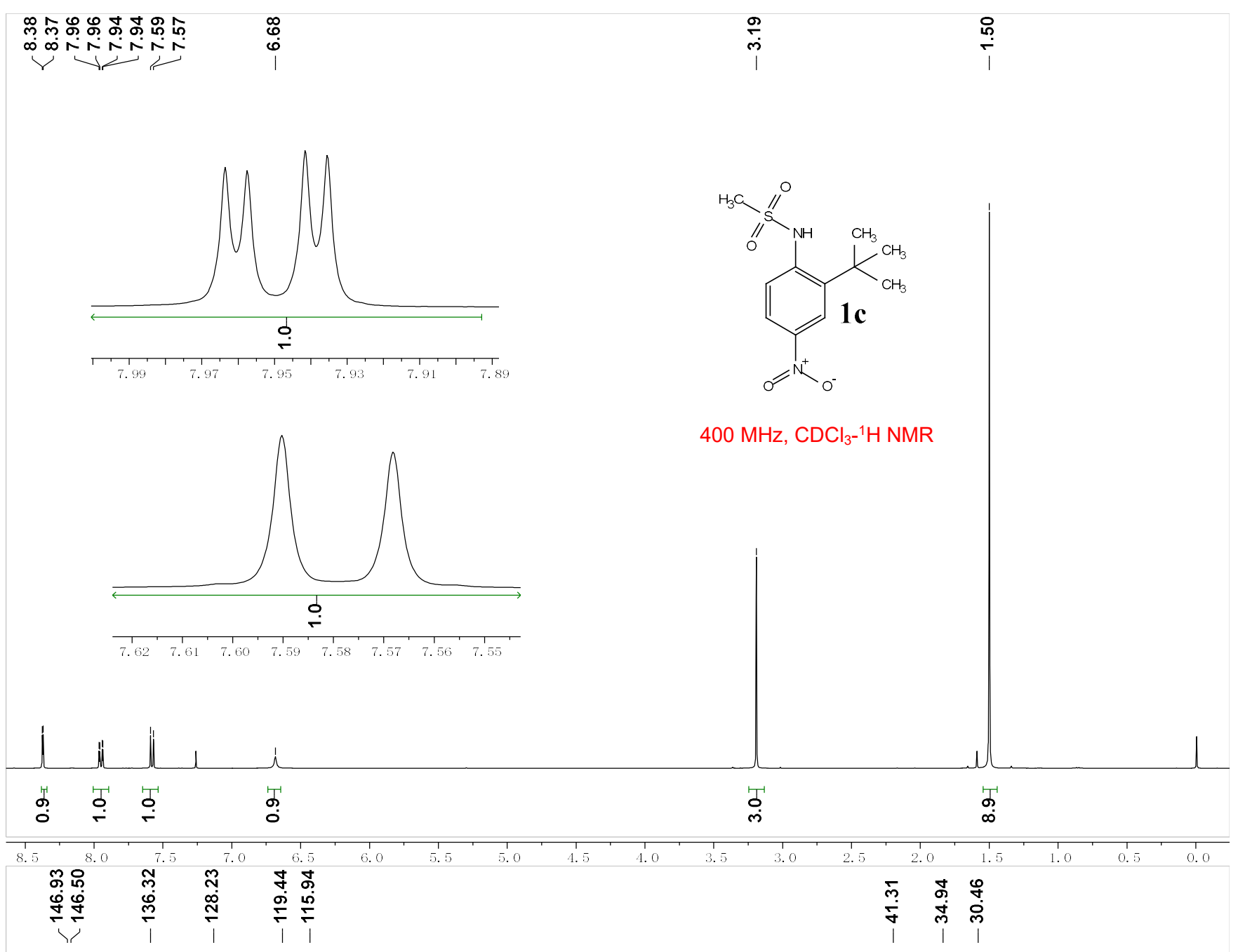

$101 \mathrm{MHz}, \mathrm{CDCl}_{3}{ }^{-13} \mathrm{C}$ NMR<smiles>CC(C)(C)c1cc([N+](=O)[O-])ccc1NS(C)(=O)=O</smiles>
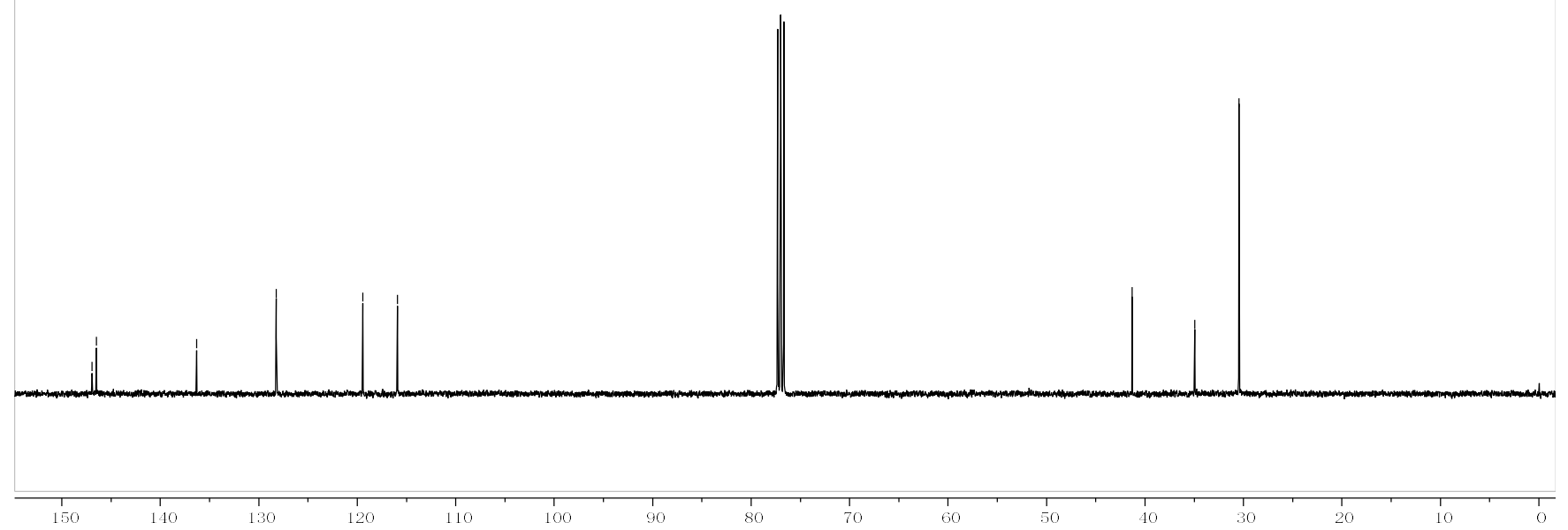

55 


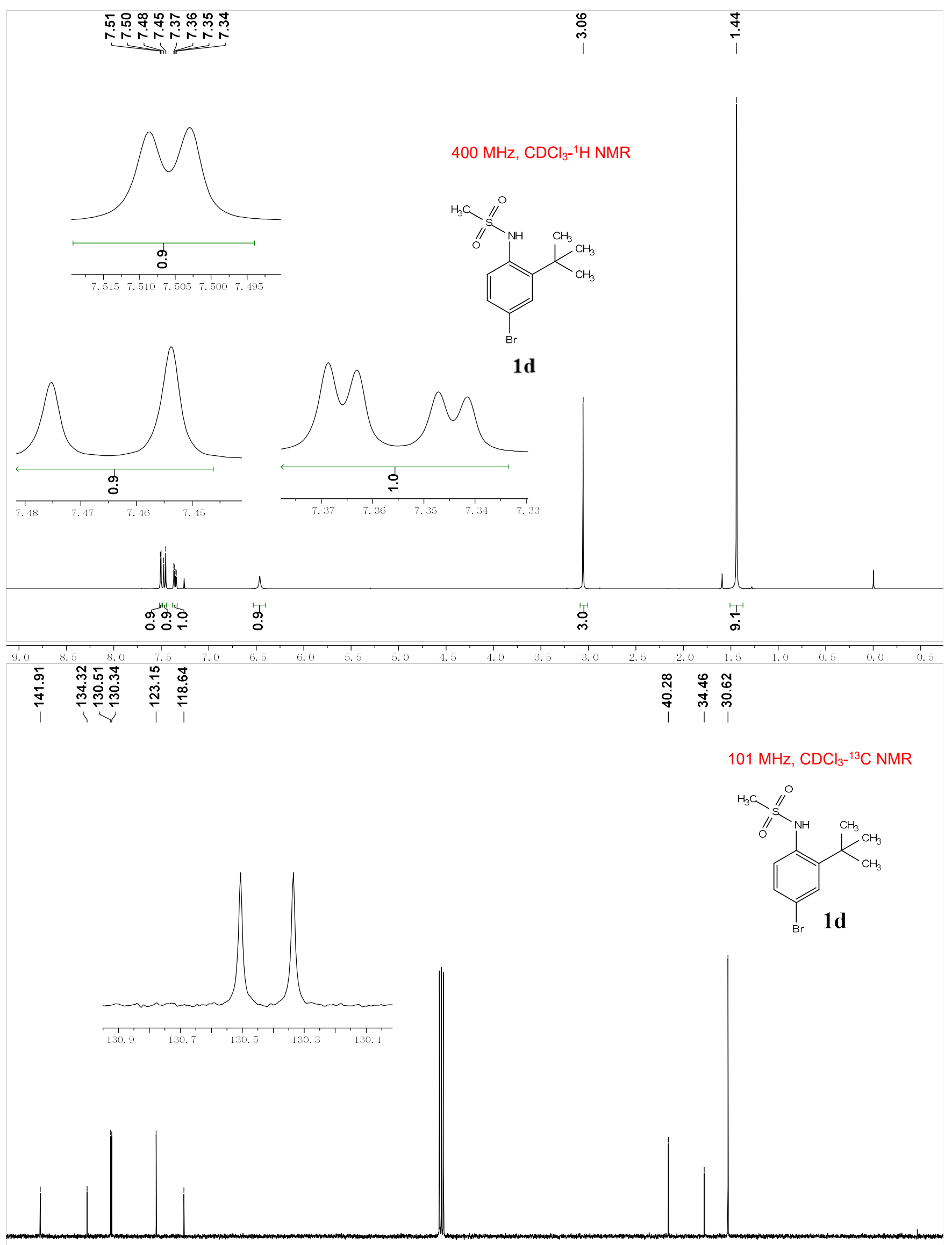


事

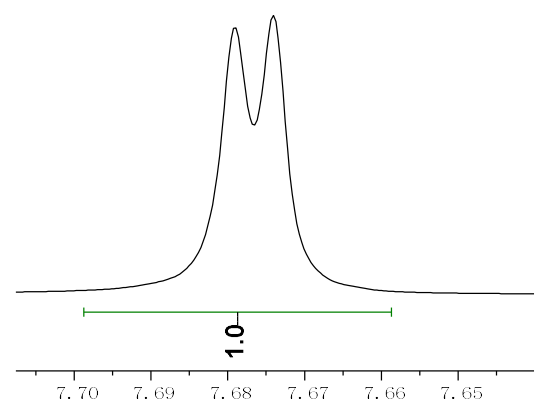

$\underset{\substack{\infty \\ \text { i }}}{\infty}$

$400 \mathrm{MHz}, \mathrm{CDCl}_{3}{ }^{-1} \mathrm{H}$ NMR

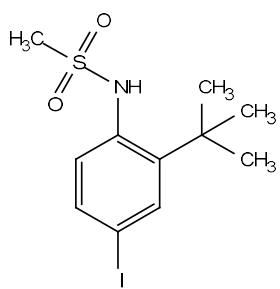

1e

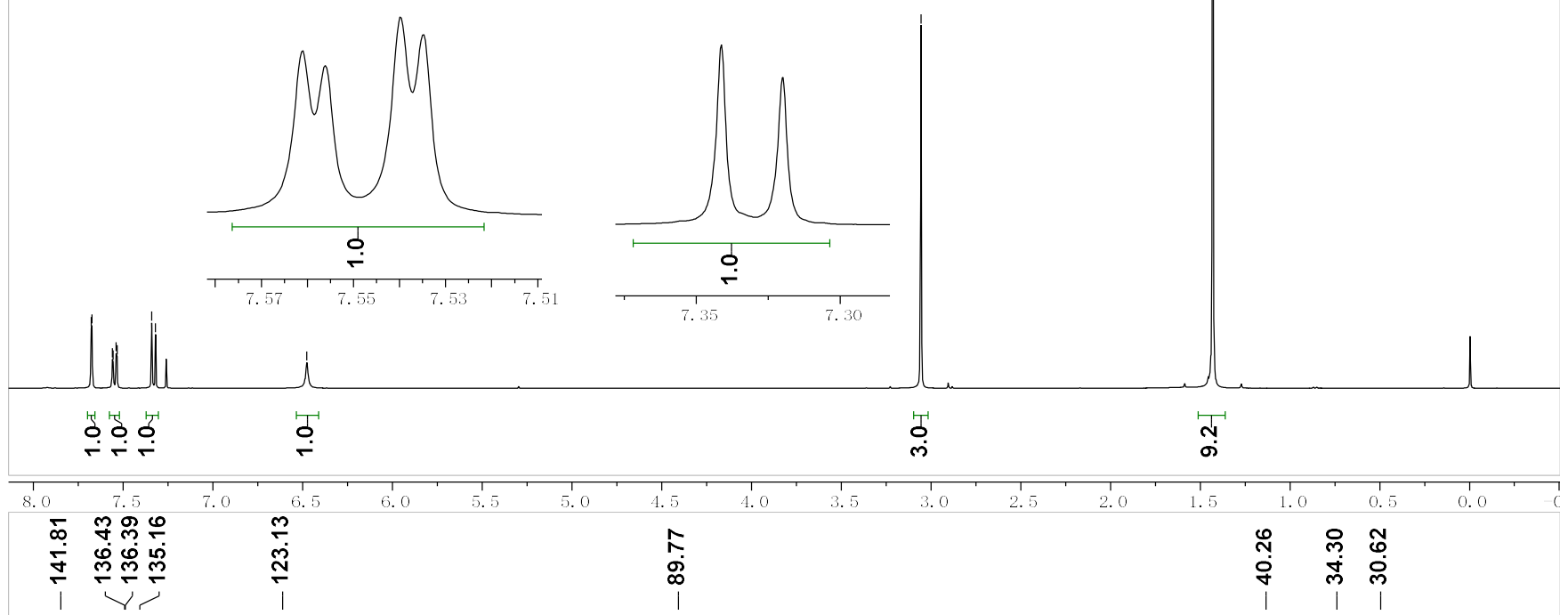

$101 \mathrm{MHz}, \mathrm{CDCl}_{3}{ }^{-13} \mathrm{C}$ NMR
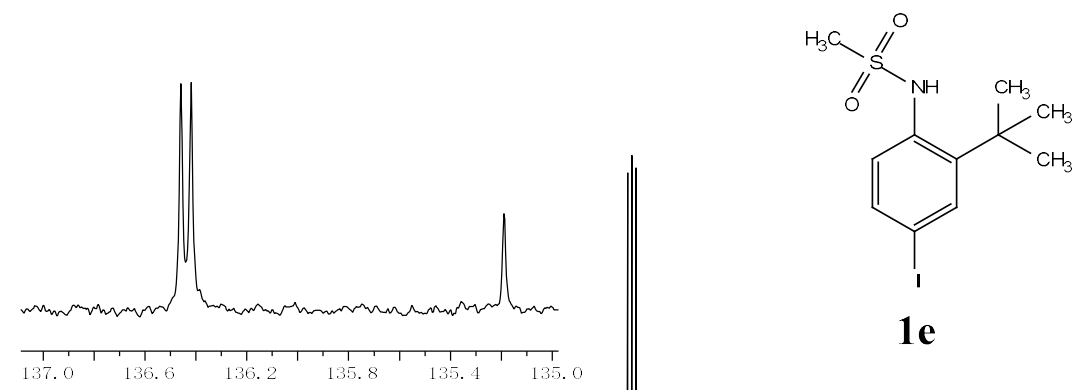

1e

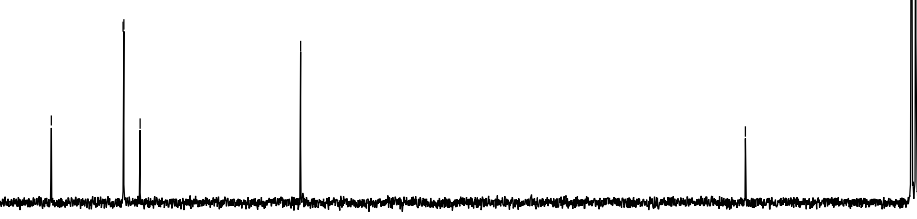


$400 \mathrm{MHz}, \mathrm{CDCl}_{3}-{ }^{1} \mathrm{H} \mathrm{NMR}$
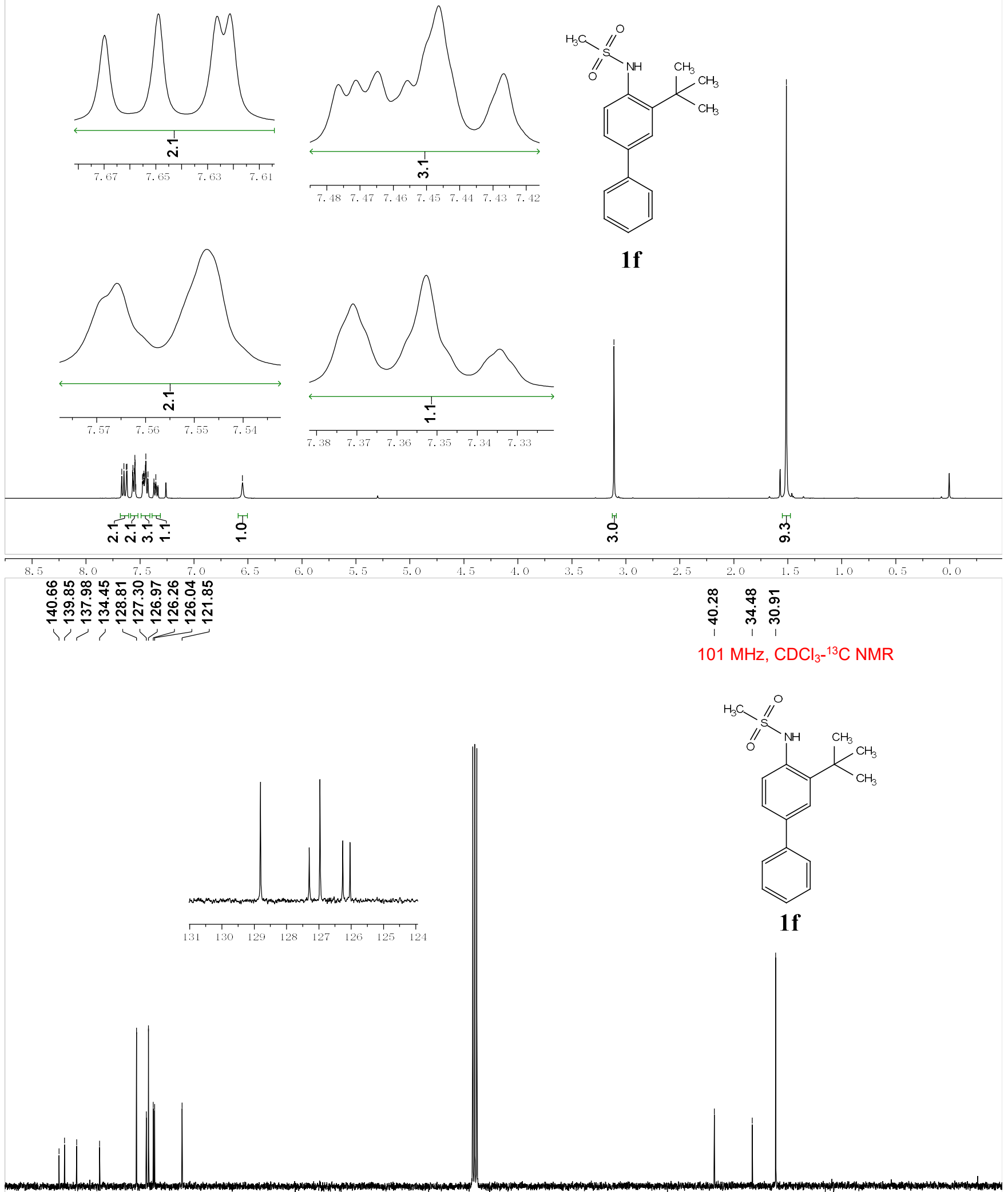

$101 \mathrm{MHz}, \mathrm{CDCl}_{3}-^{-13} \mathrm{C}$ NMR 


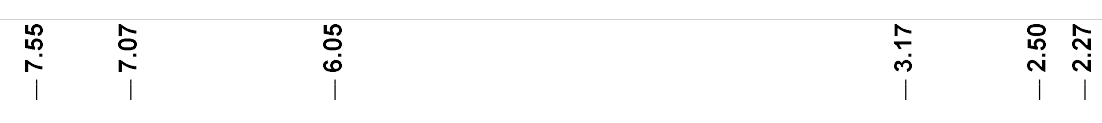

$400 \mathrm{MHz}, \mathrm{CDCl}_{3}{ }^{-1} \mathrm{H} \mathrm{NMR}$

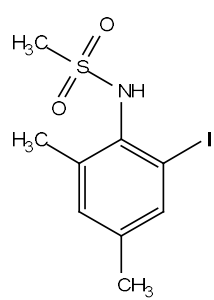

19

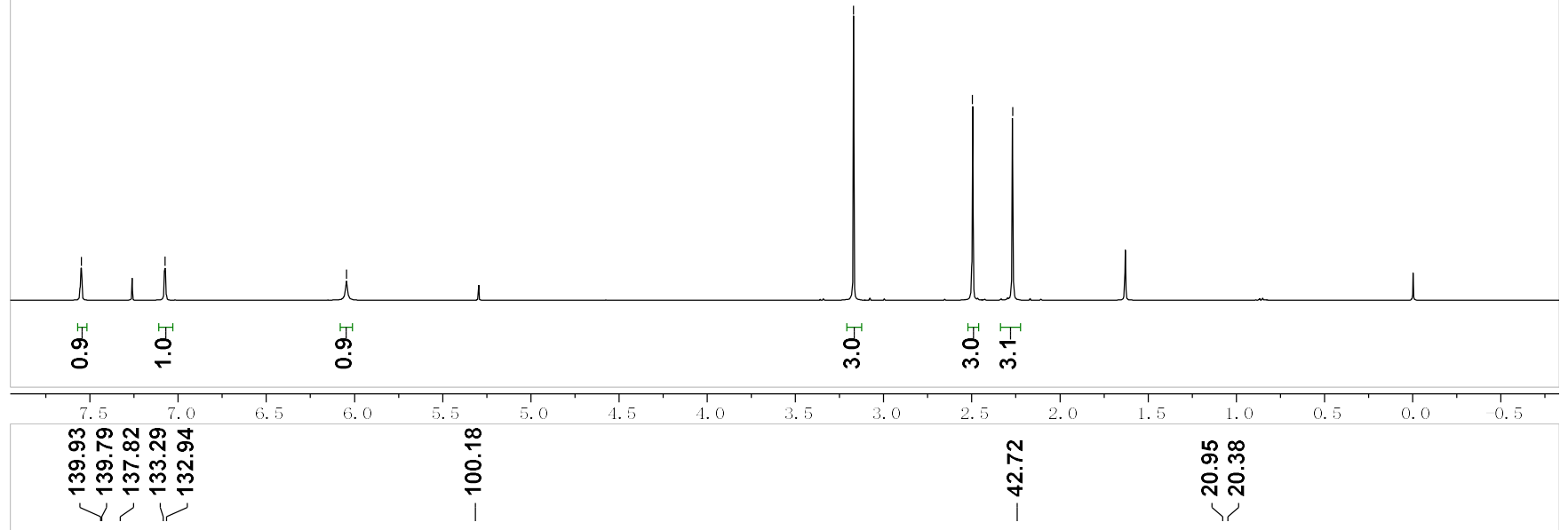

$101 \mathrm{MHz}, \mathrm{CDCl}_{3}-^{13} \mathrm{C}$ NMR
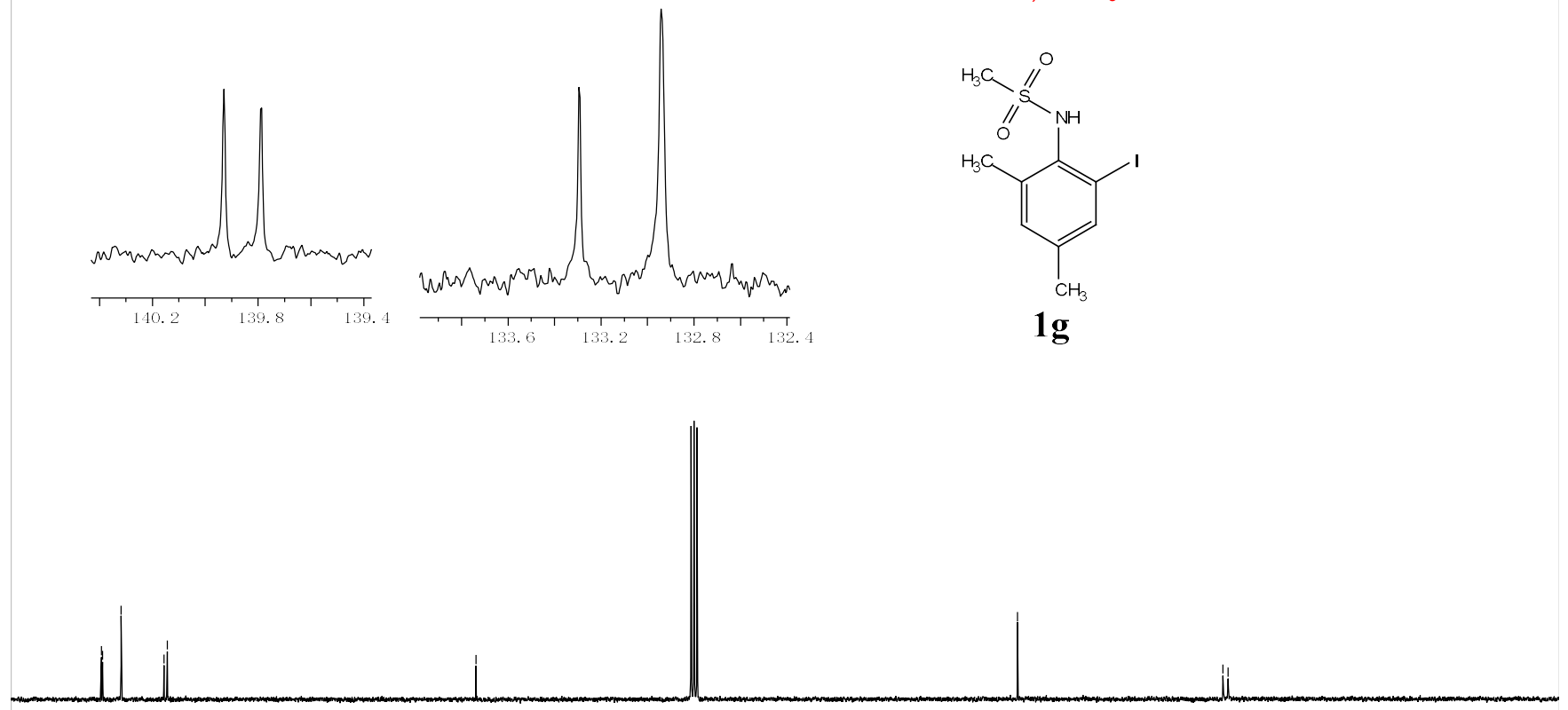

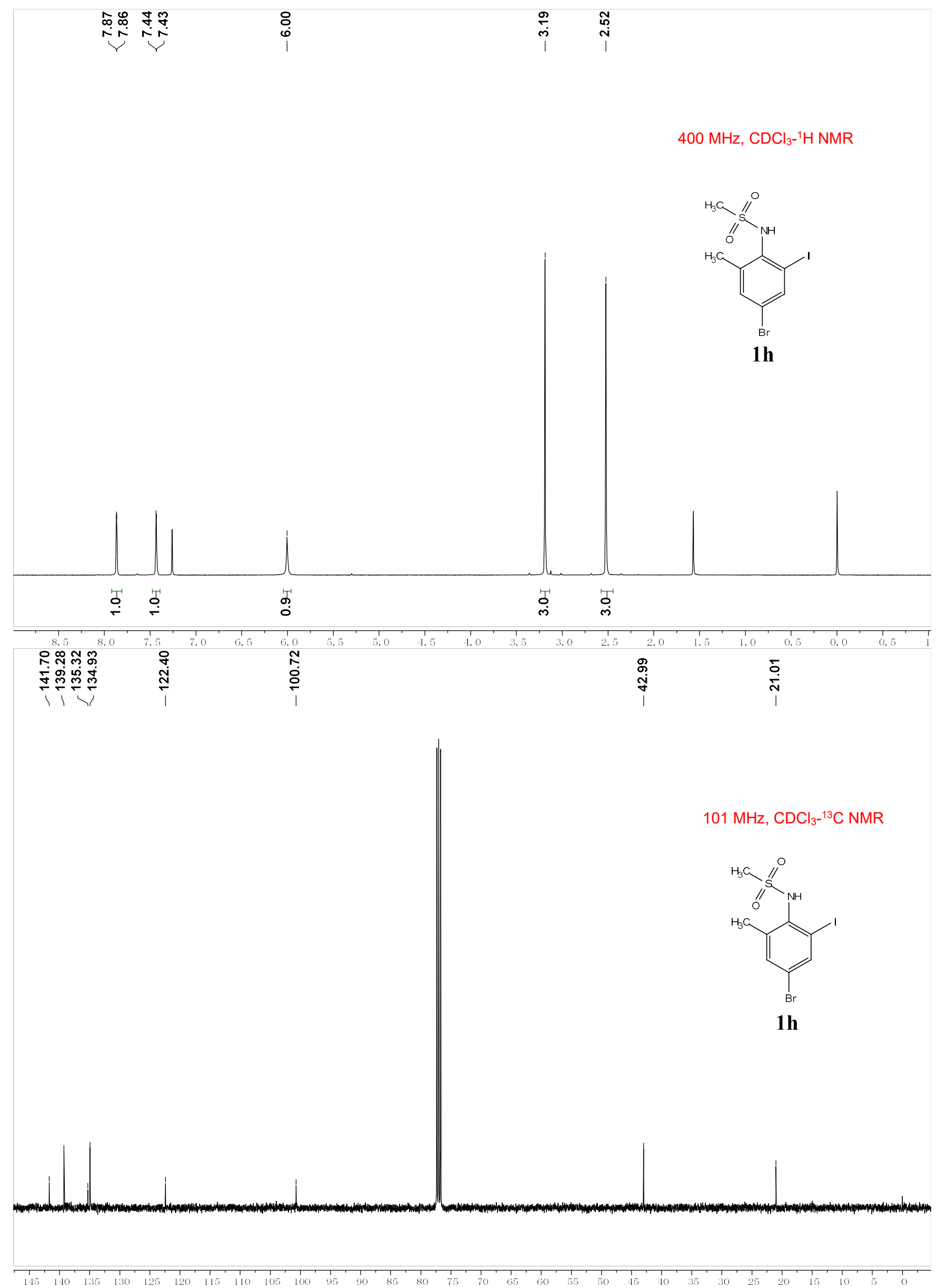


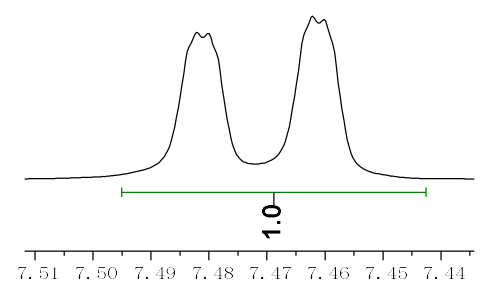

$400 \mathrm{MHz}, \mathrm{CDCl}_{3}-{ }^{-1} \mathrm{H}$ NMR
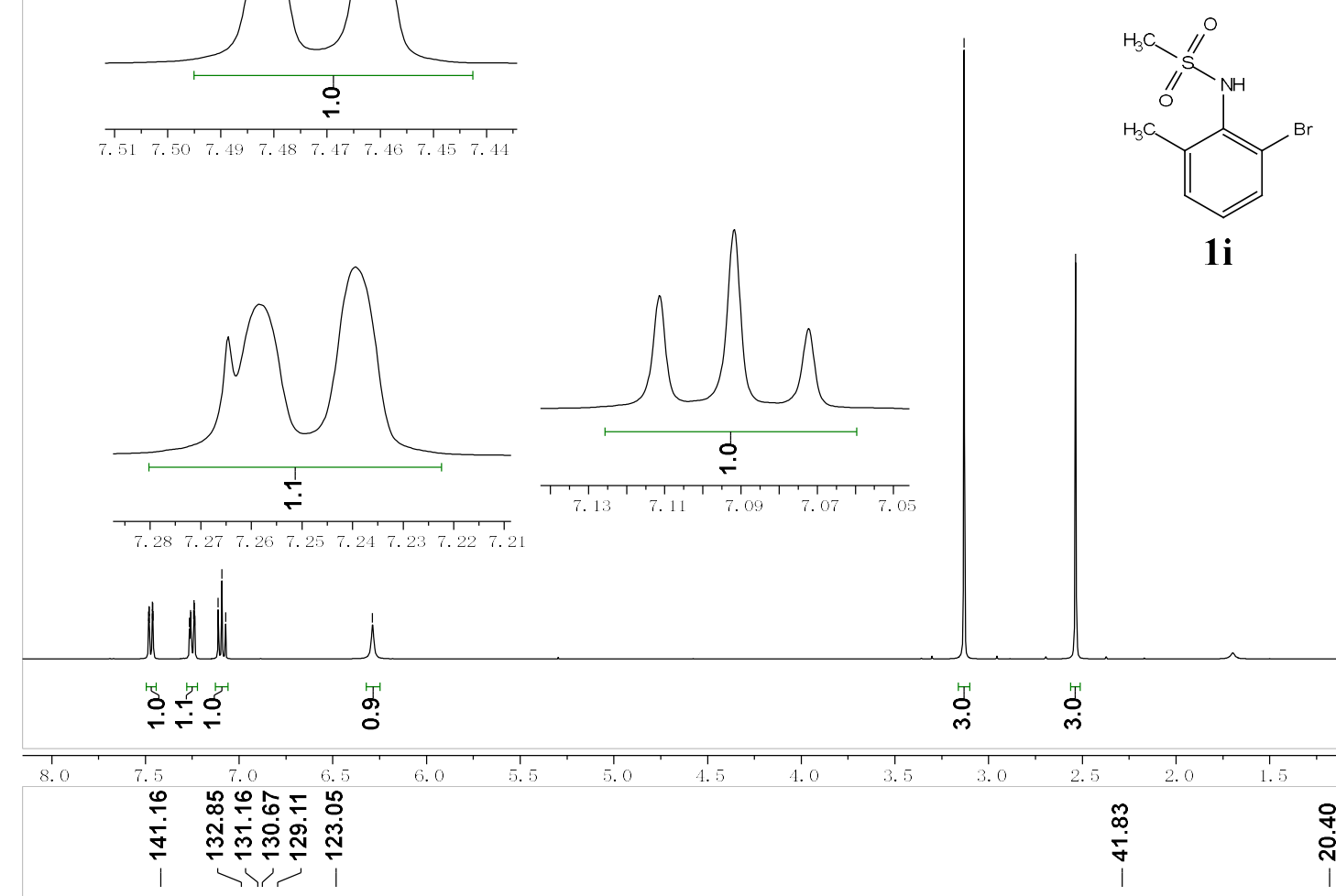

$101 \mathrm{MHz}, \mathrm{CDCl}_{3}{ }^{-13} \mathrm{C} \mathrm{NMR}$
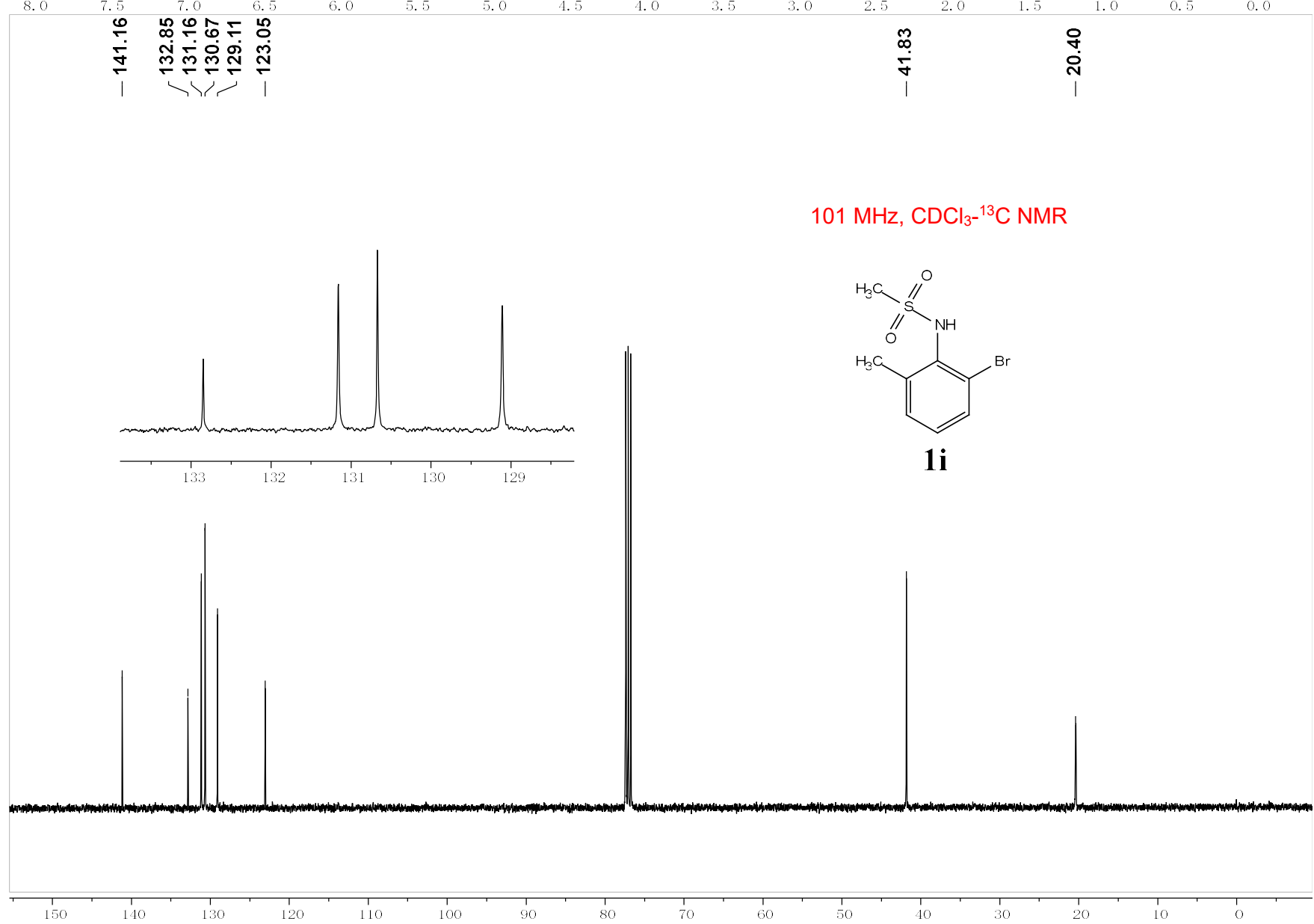

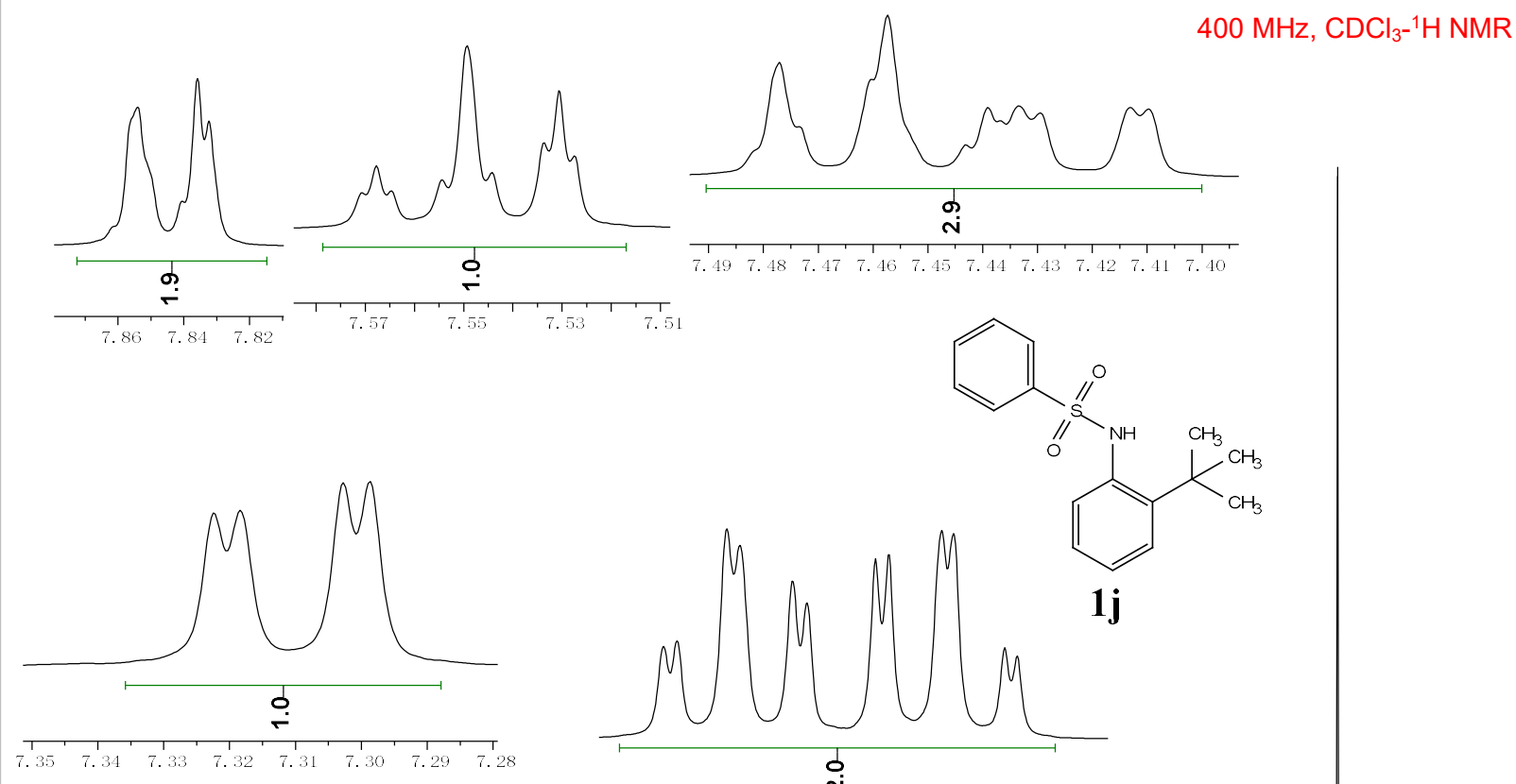

Williditici

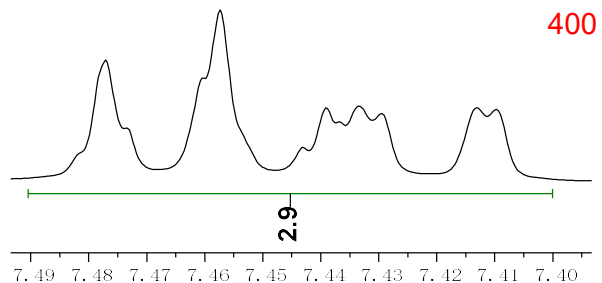

$00 \mathrm{MHz}, \mathrm{CDCl}_{3}-{ }^{1} \mathrm{H} \mathrm{NMR}$

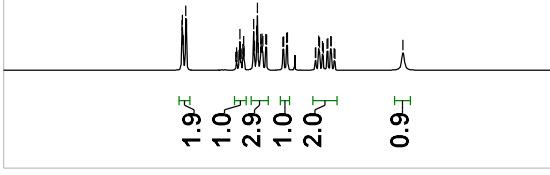

웅

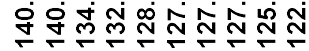

r.
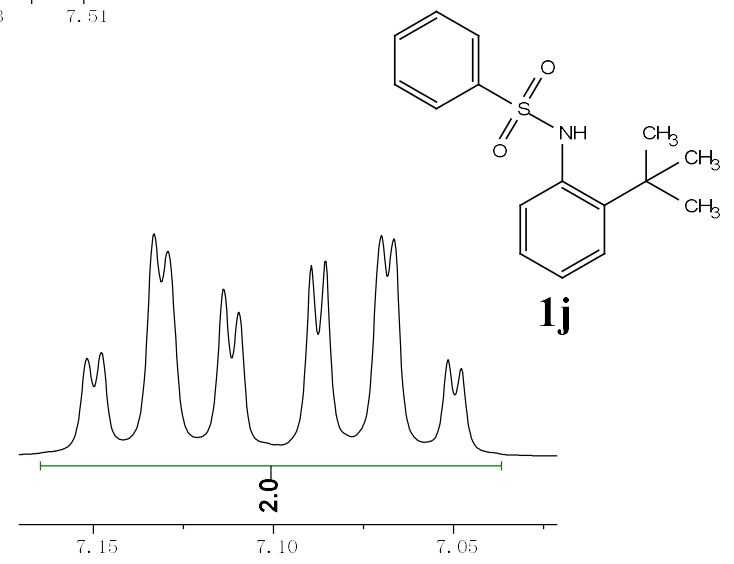

$1 \mathrm{j}$

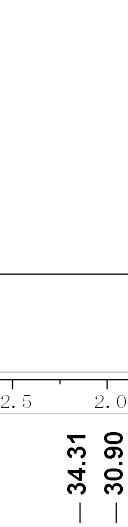

$101 \mathrm{MHz}, \mathrm{CDCl}_{3}-{ }^{13} \mathrm{C}$ NMR
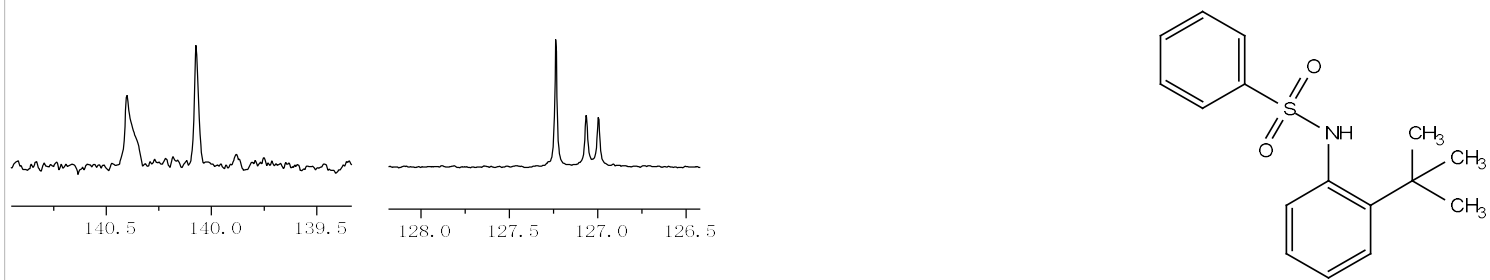

$1 \mathbf{j}$
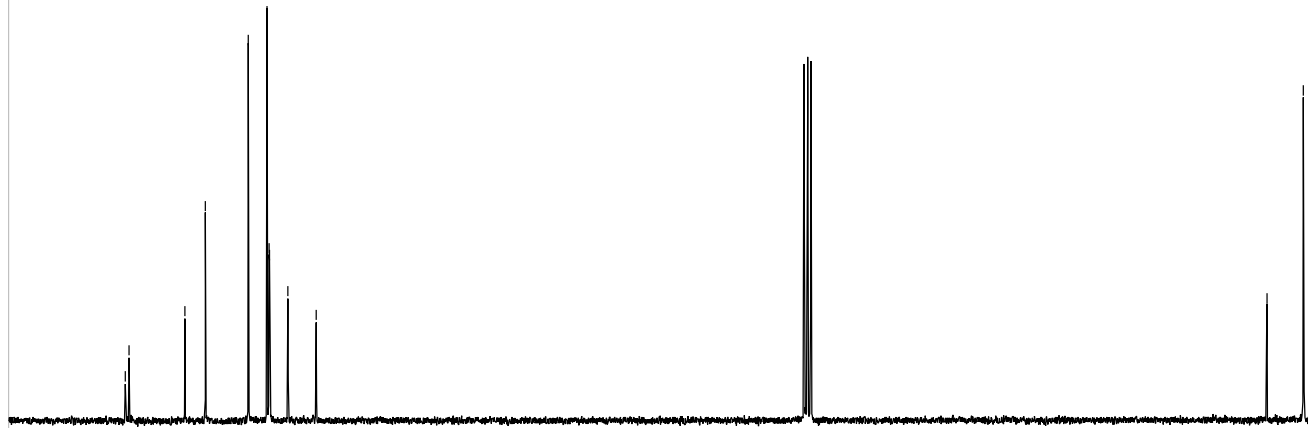


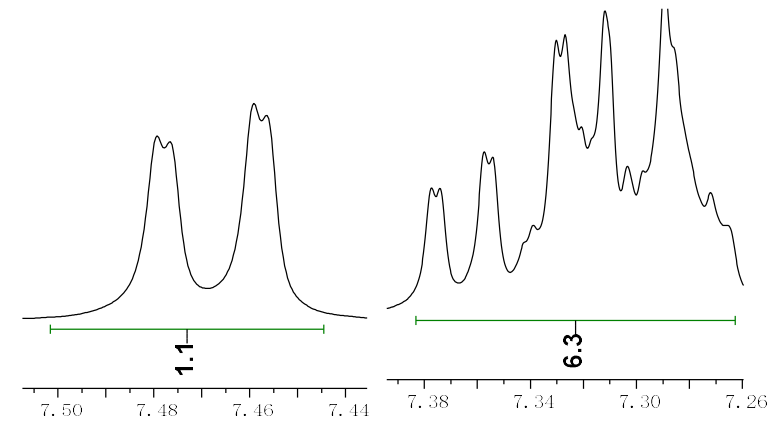

$400 \mathrm{MHz}, \mathrm{CDCl}_{3}-{ }^{1} \mathrm{H} \mathrm{NMR}$
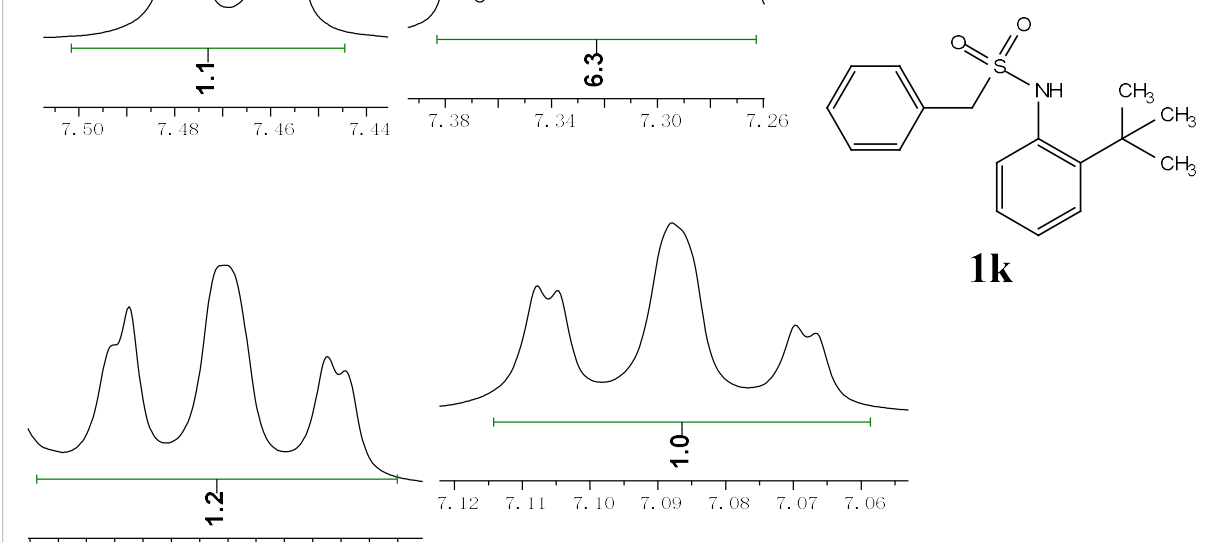

$1 \mathbf{k}$
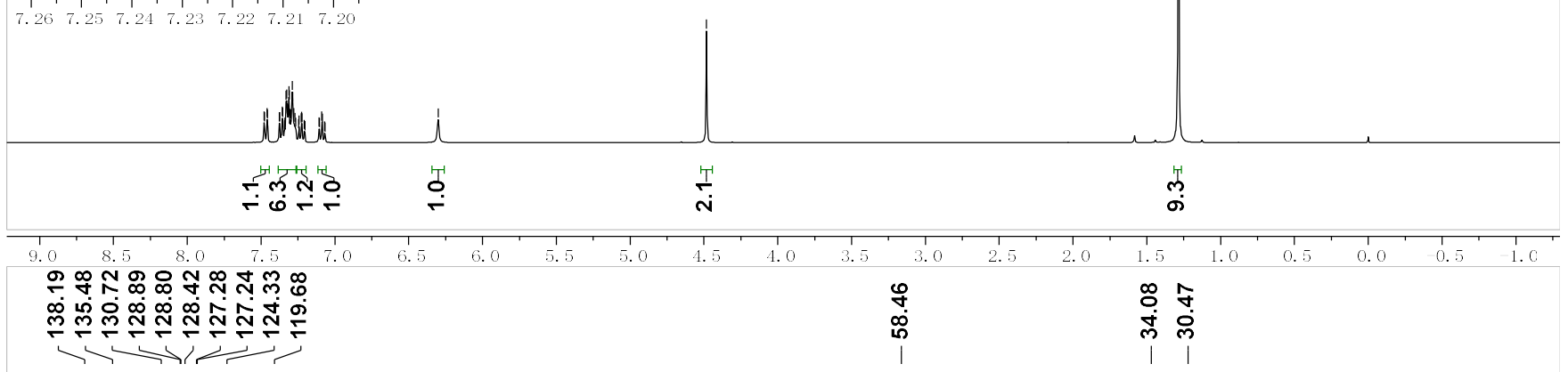

$101 \mathrm{MHz}, \mathrm{CDCl}_{3} \mathbf{-}^{13} \mathrm{C}$ NMR
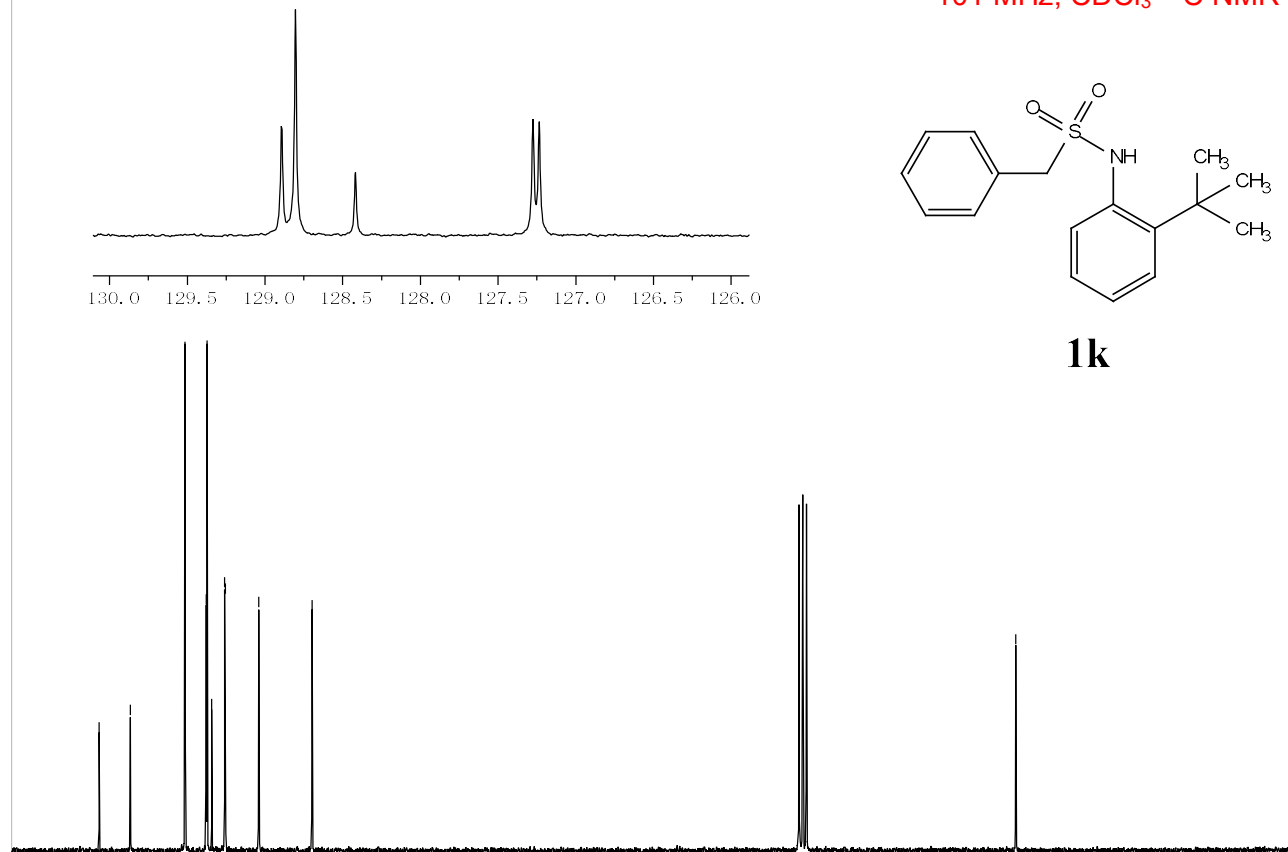

$1 \mathrm{k}$

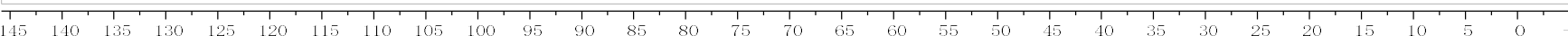




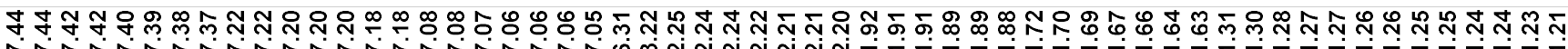

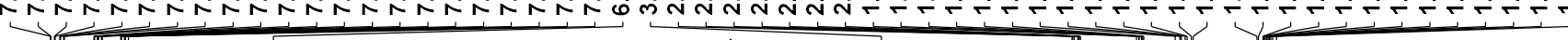

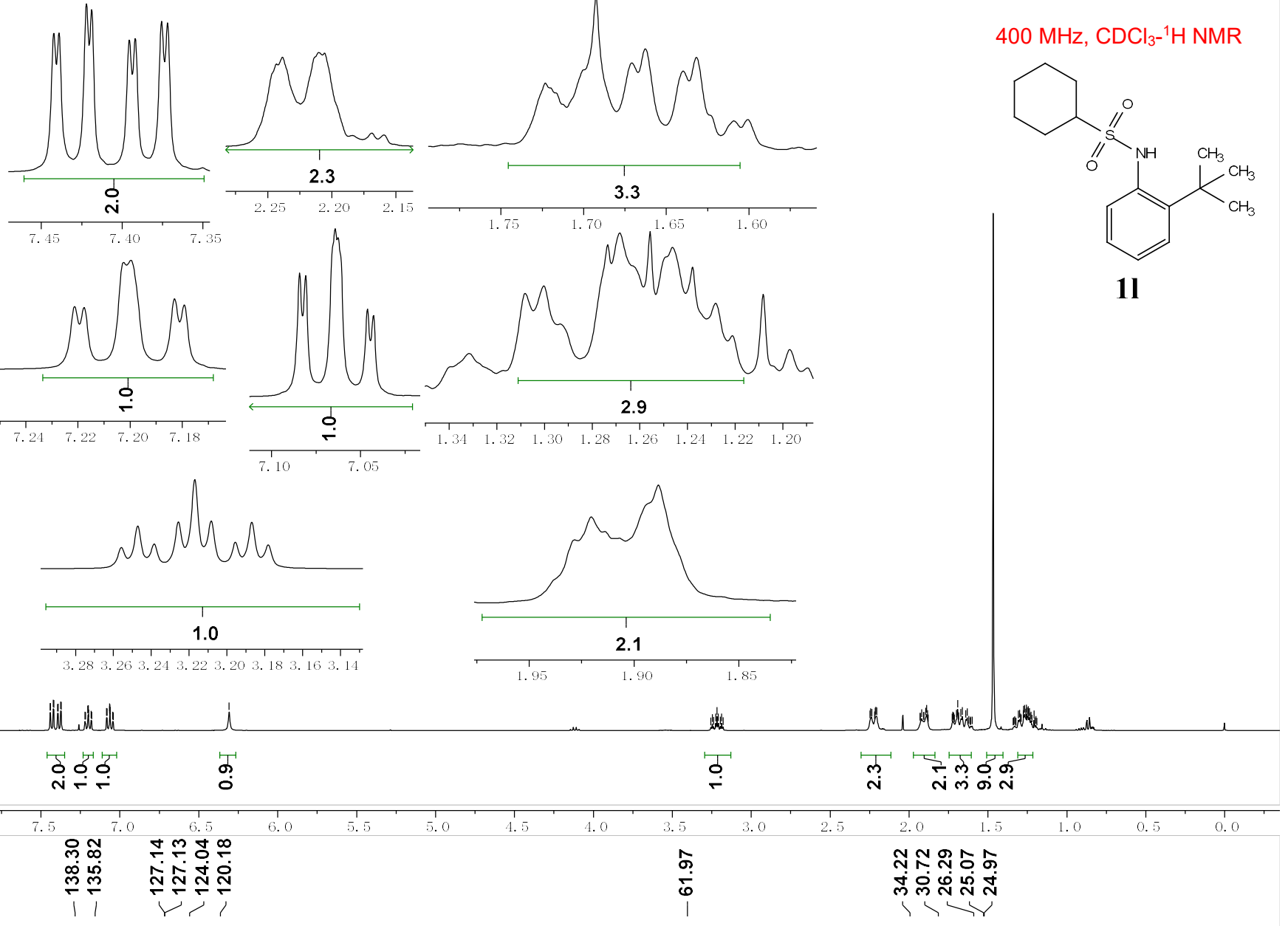

$101 \mathrm{MHz}, \mathrm{CDCl}_{3}{ }^{-13} \mathrm{C}$ NMR
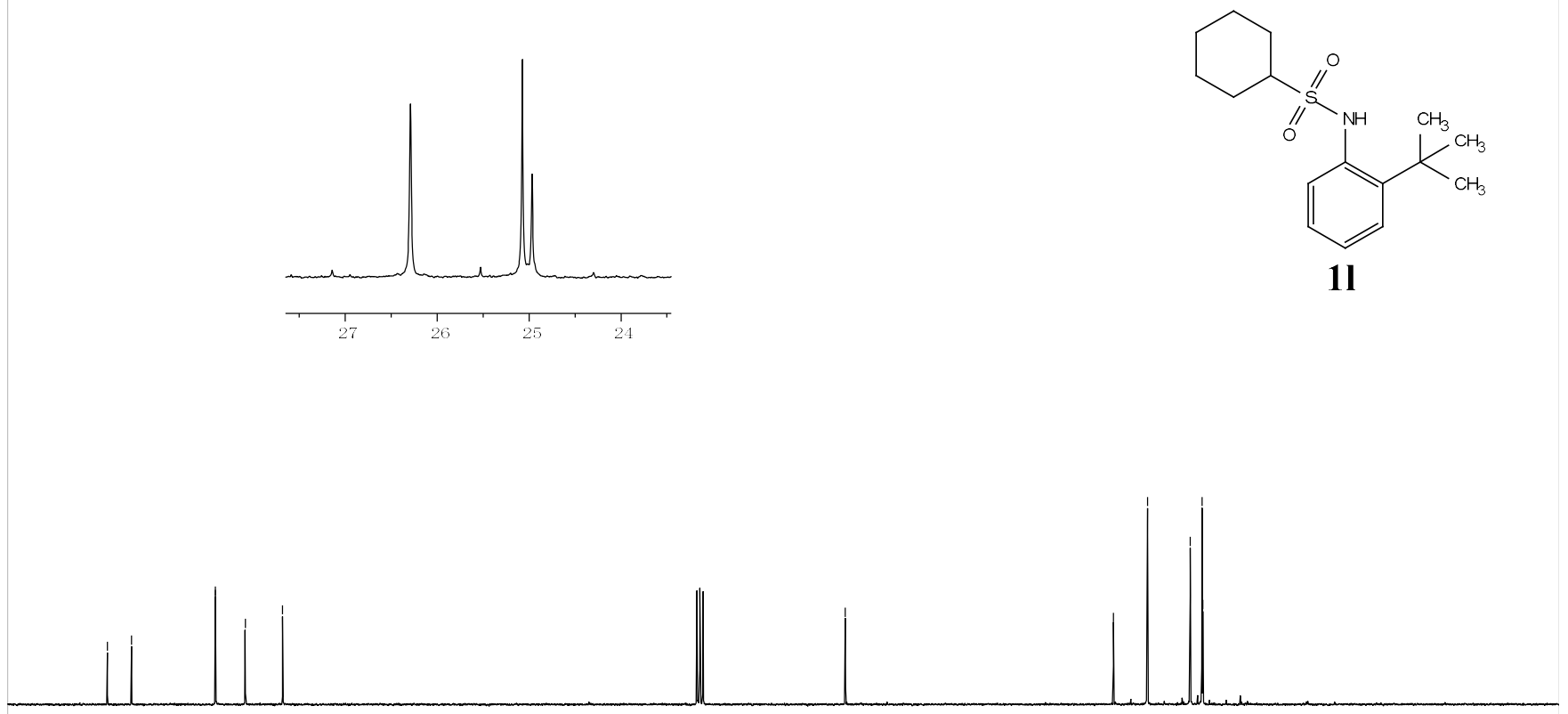

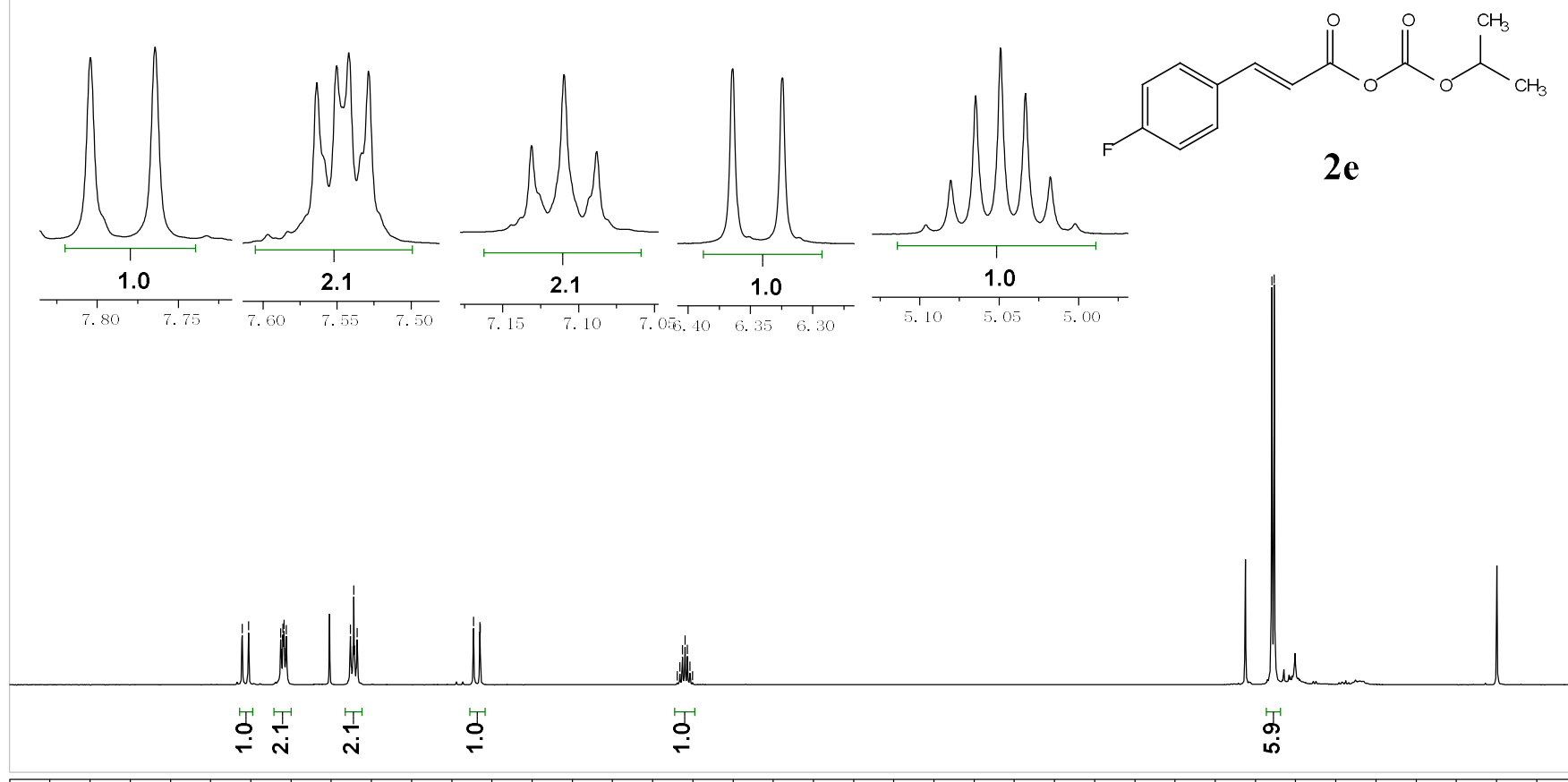

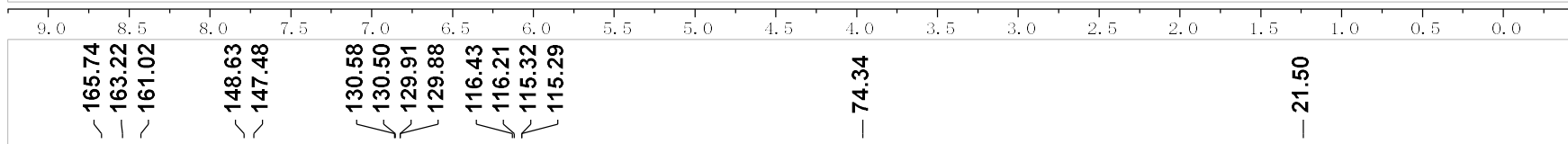

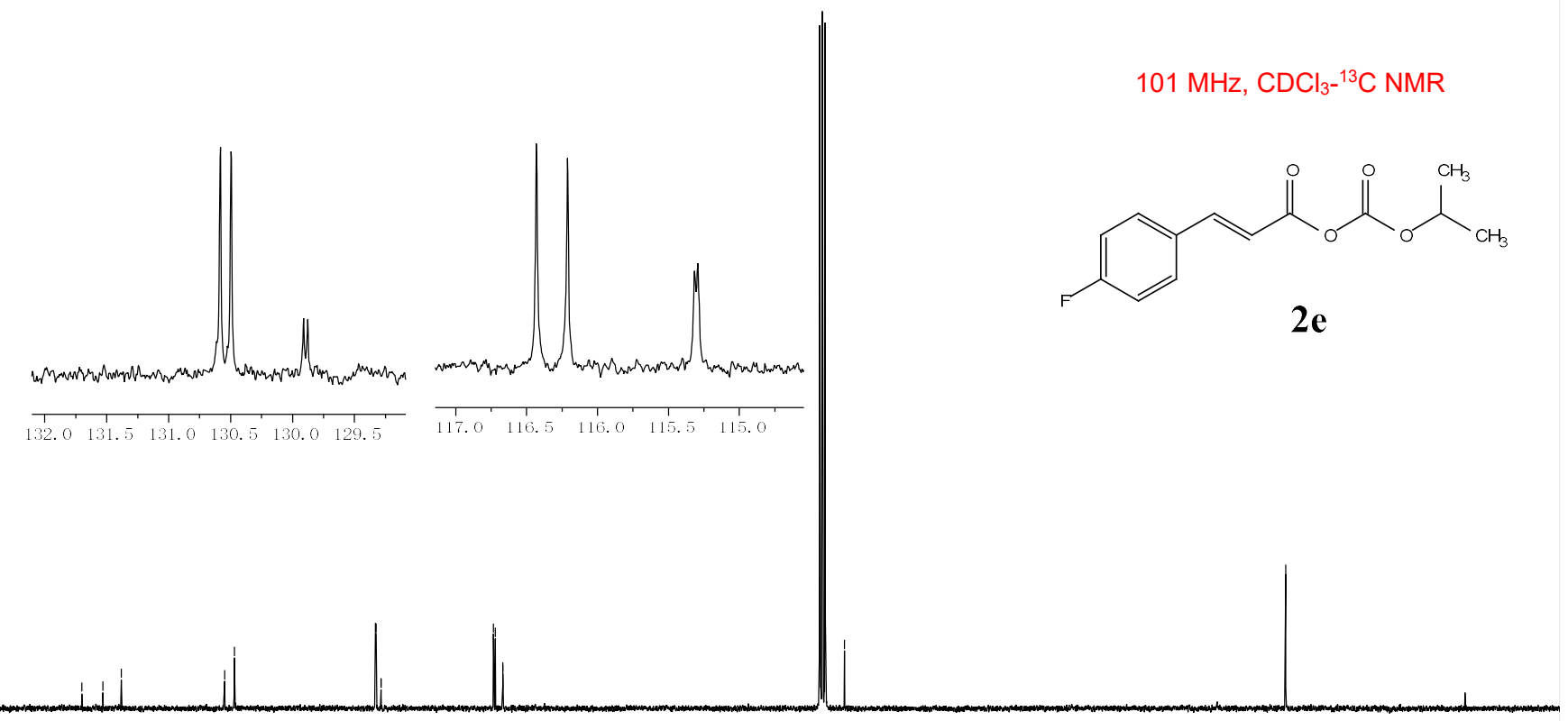


$376 \mathrm{MHz}, \mathrm{CDCl}_{3}-^{-19} \mathrm{~F} \mathrm{NMR}$
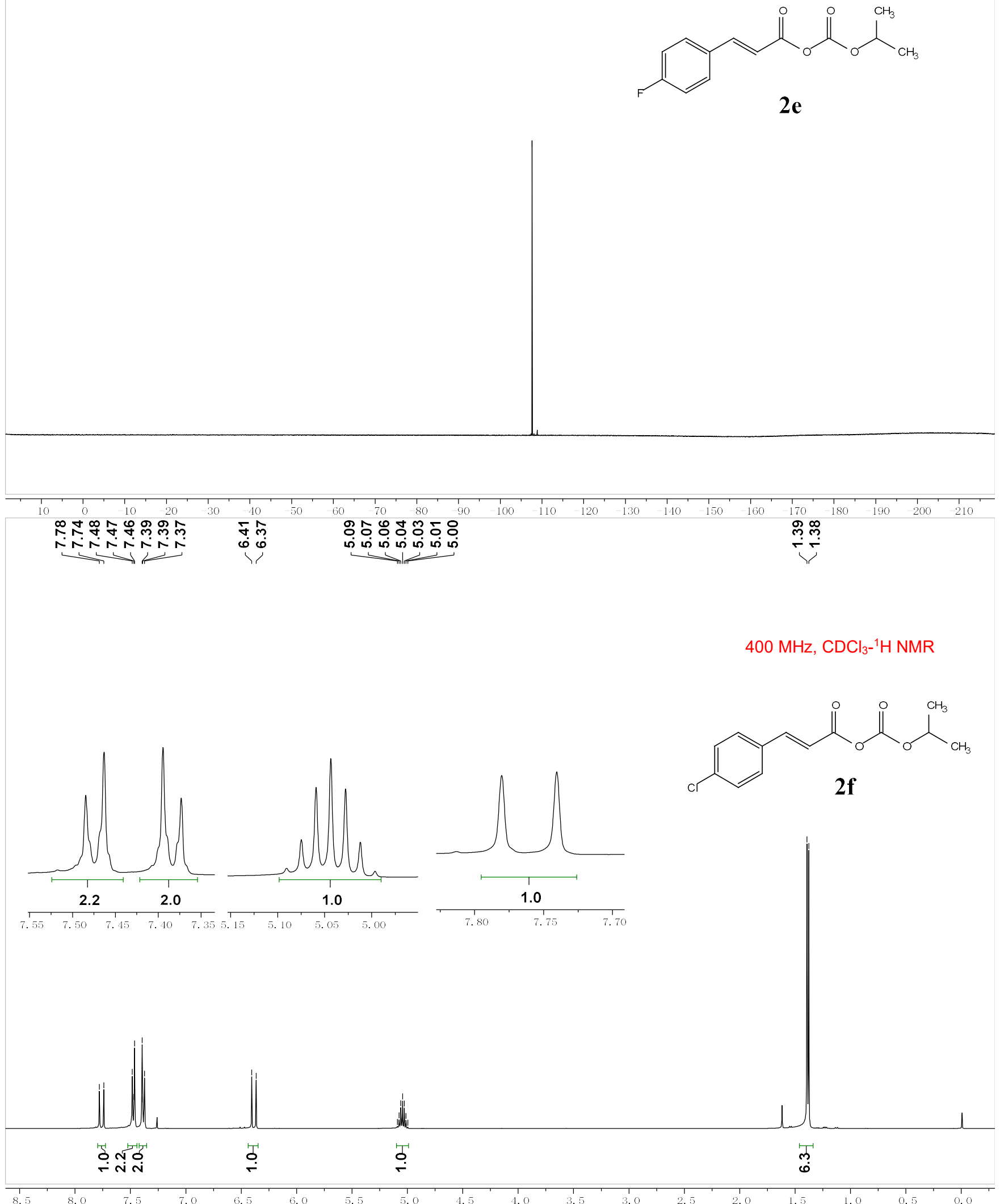
$101 \mathrm{MHz}, \mathrm{CDCl}_{3}{ }^{-13} \mathrm{C}$ NMR
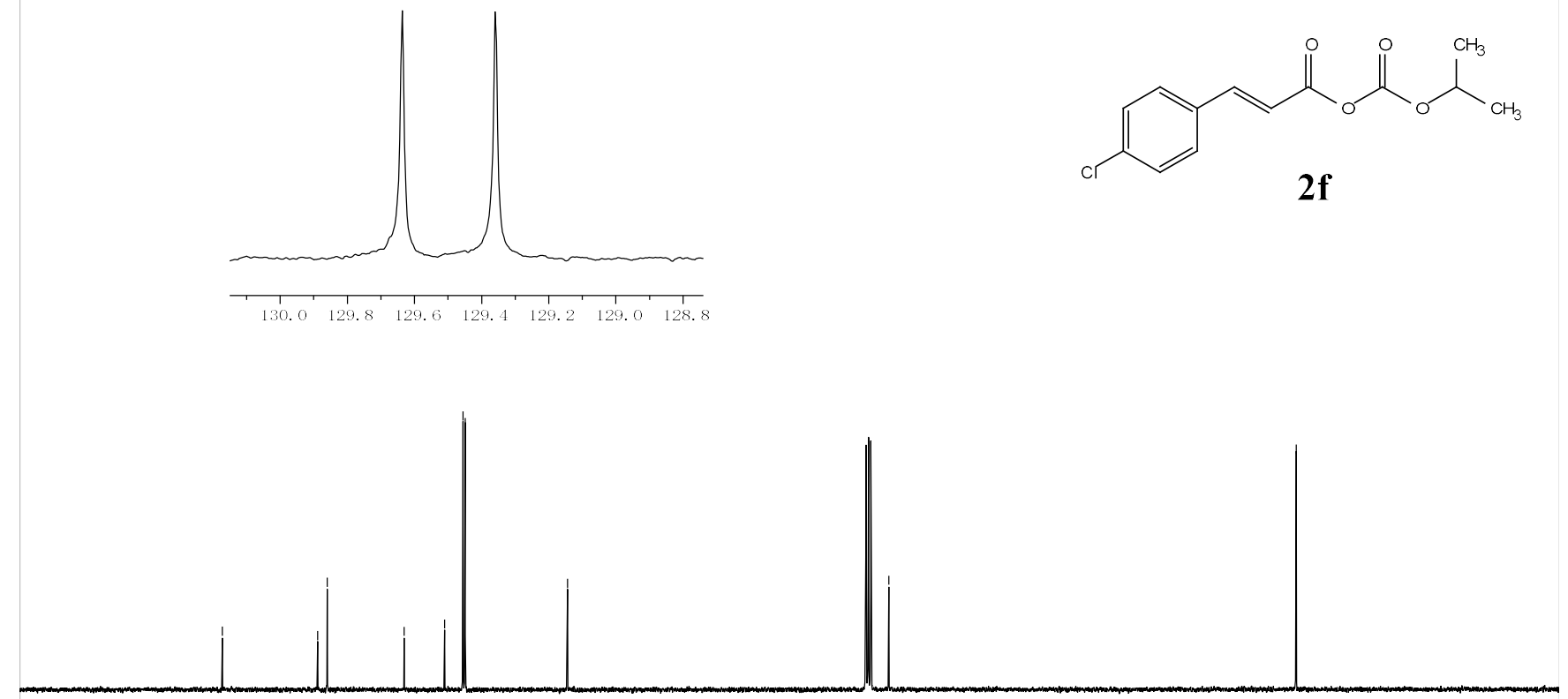

$400 \mathrm{MHz}, \mathrm{CDCl}_{3}-{ }^{-1} \mathrm{H}$ NMR
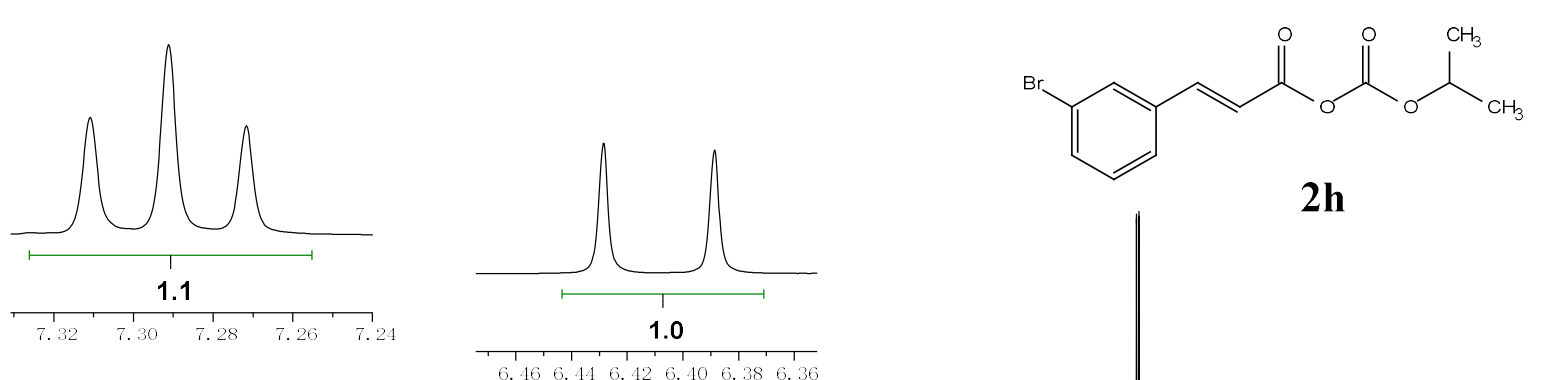

$2 \mathbf{h}$

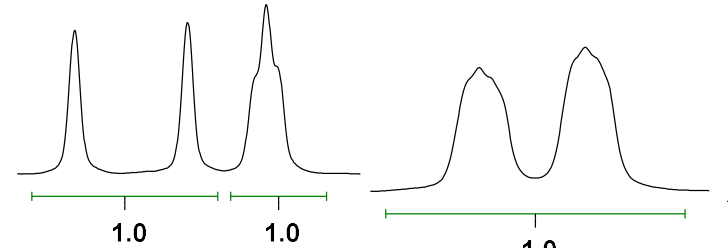

$\begin{array}{lllllllll}7.76 & 7.74 & 7.72 & 7.70 & 7.68 & 7.66\end{array}$
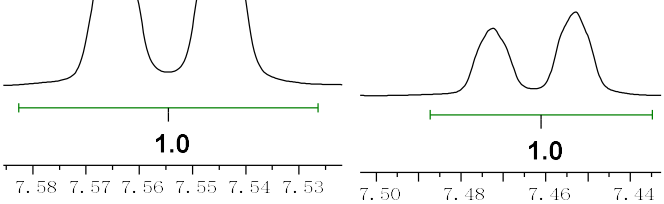

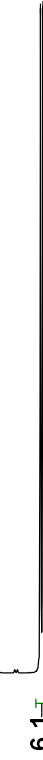

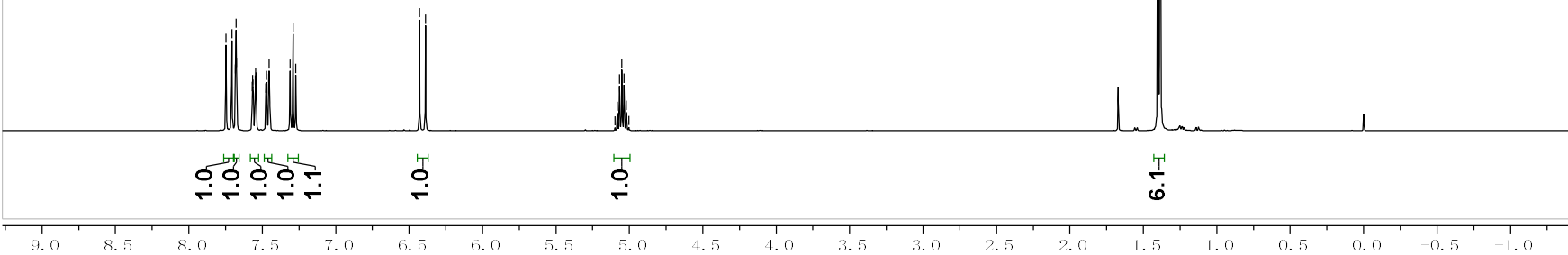



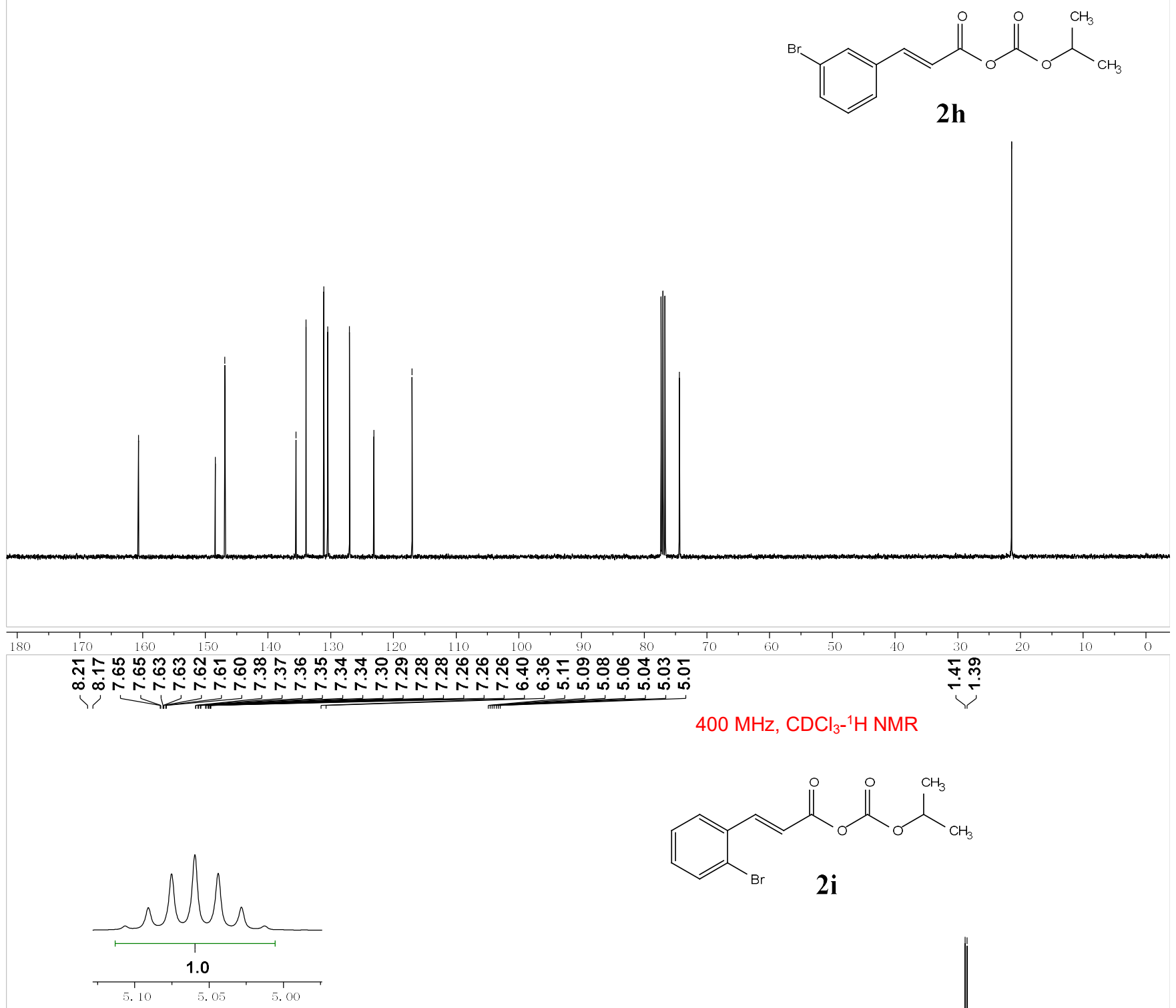<smiles>[Y]=C(C=Cc1ccccc1Br)OC(=O)OC(C)C</smiles>
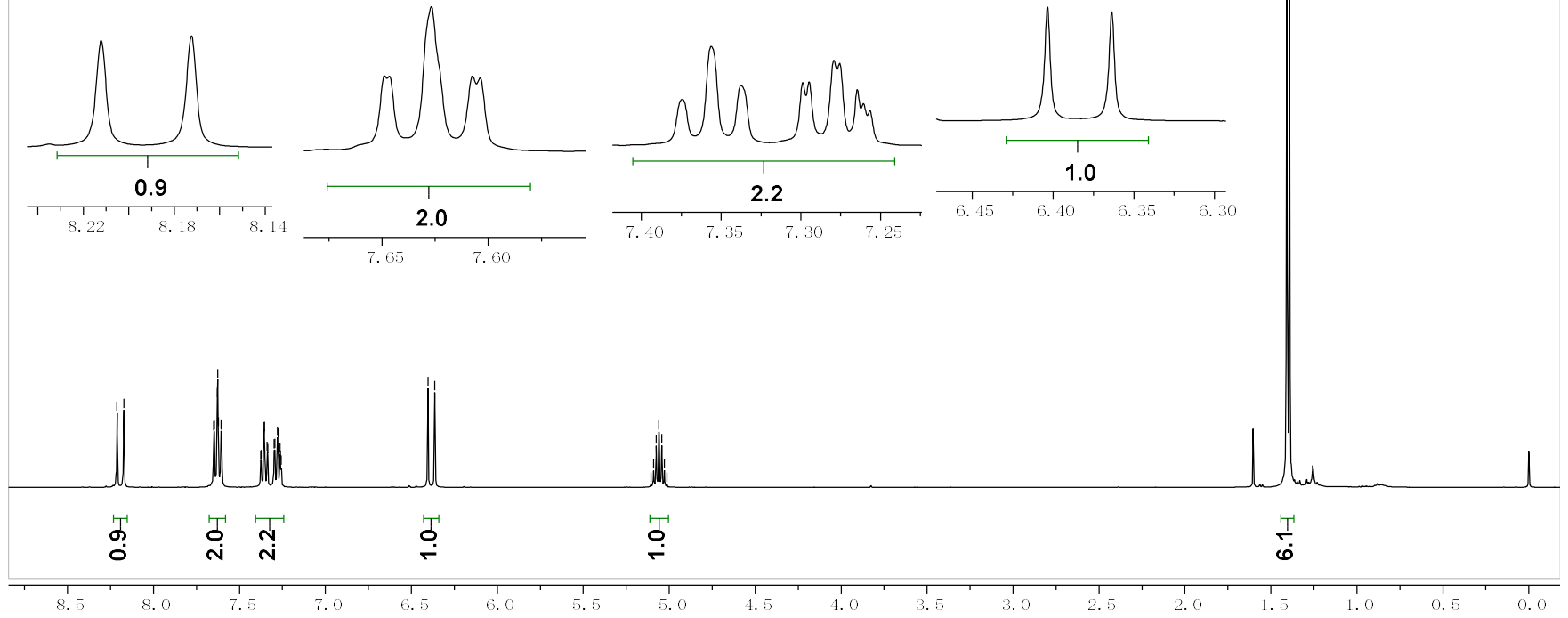

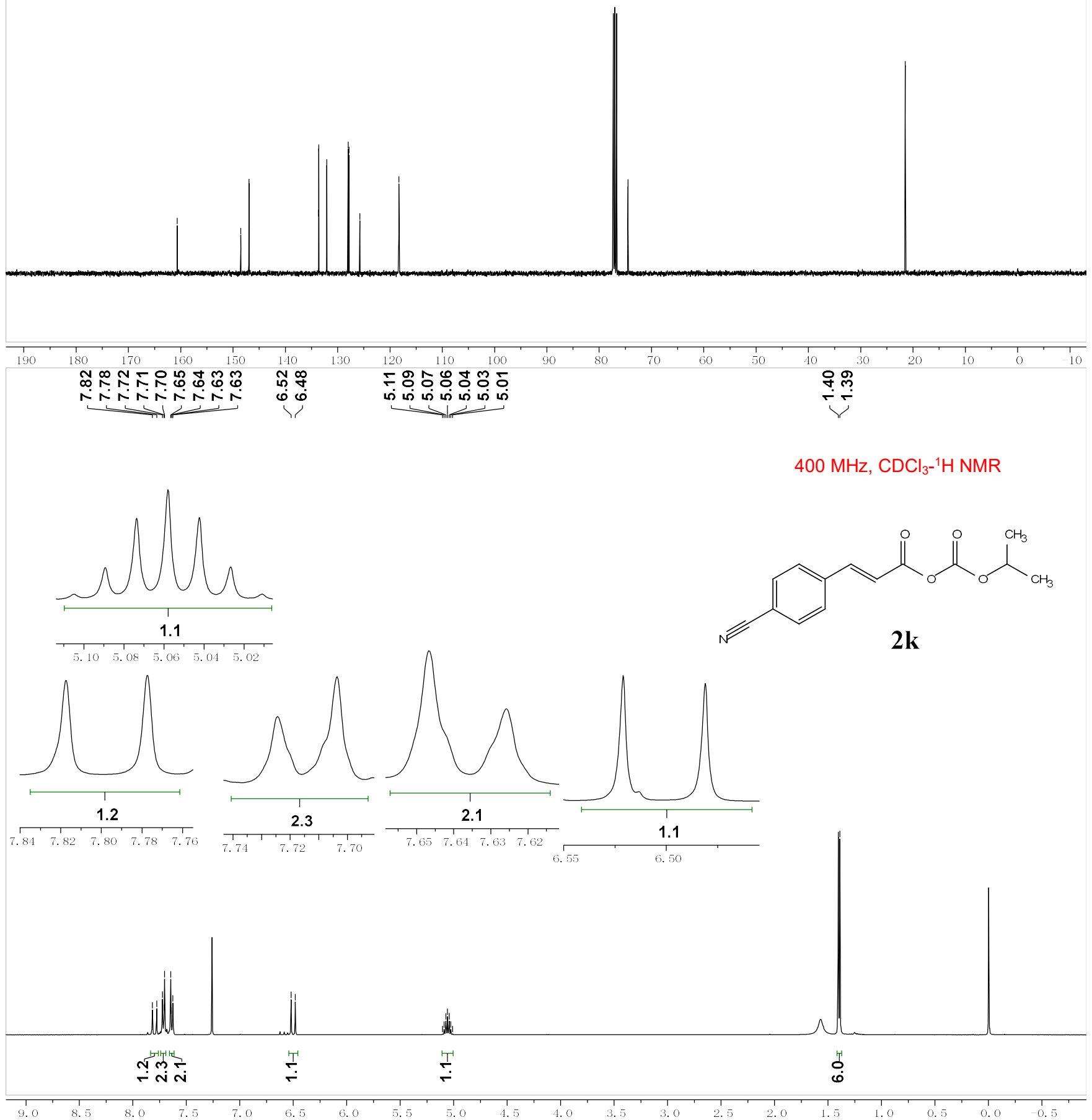

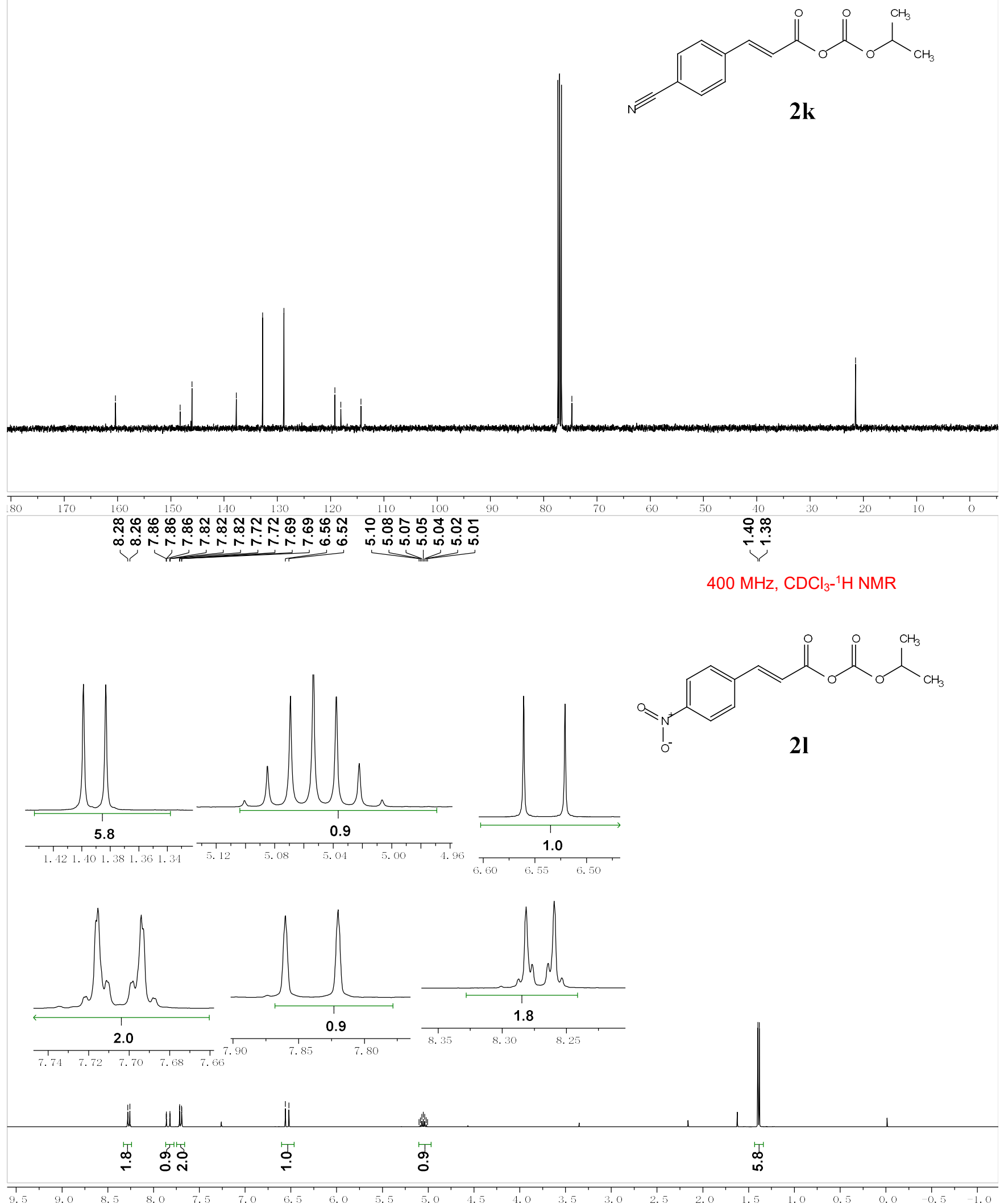
I

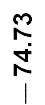

$\stackrel{\substack{i \\ \hdashline}}{\operatorname{s}}$

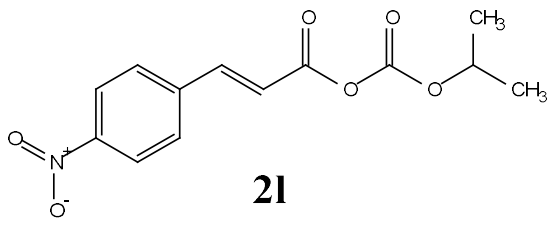

$101 \mathrm{MHz}, \mathrm{CDCl}_{3}-^{13} \mathrm{C}$ NMR

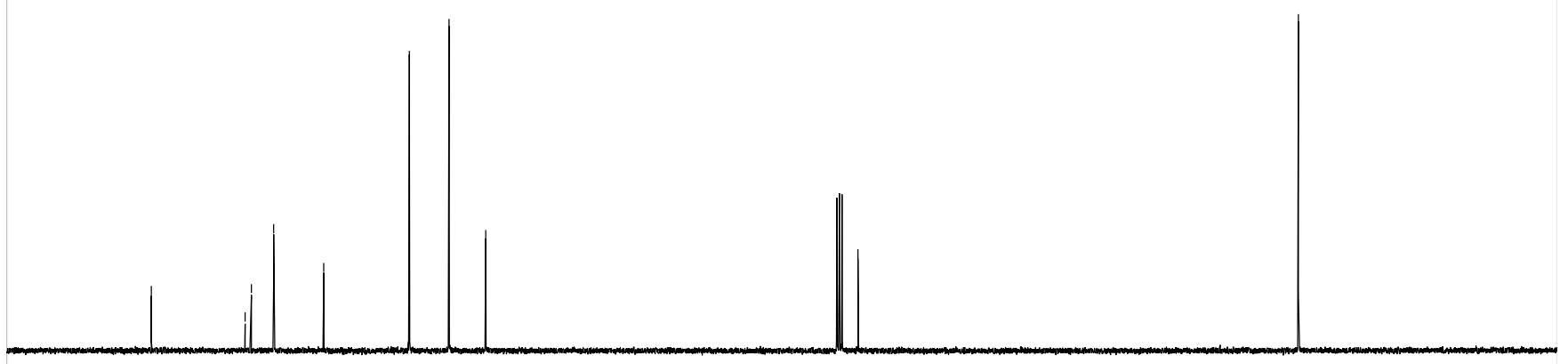

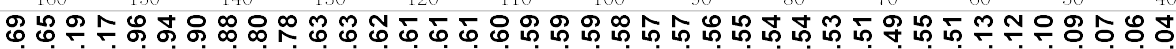

党

$\stackrel{\sqrt[3]{+}}{?}$
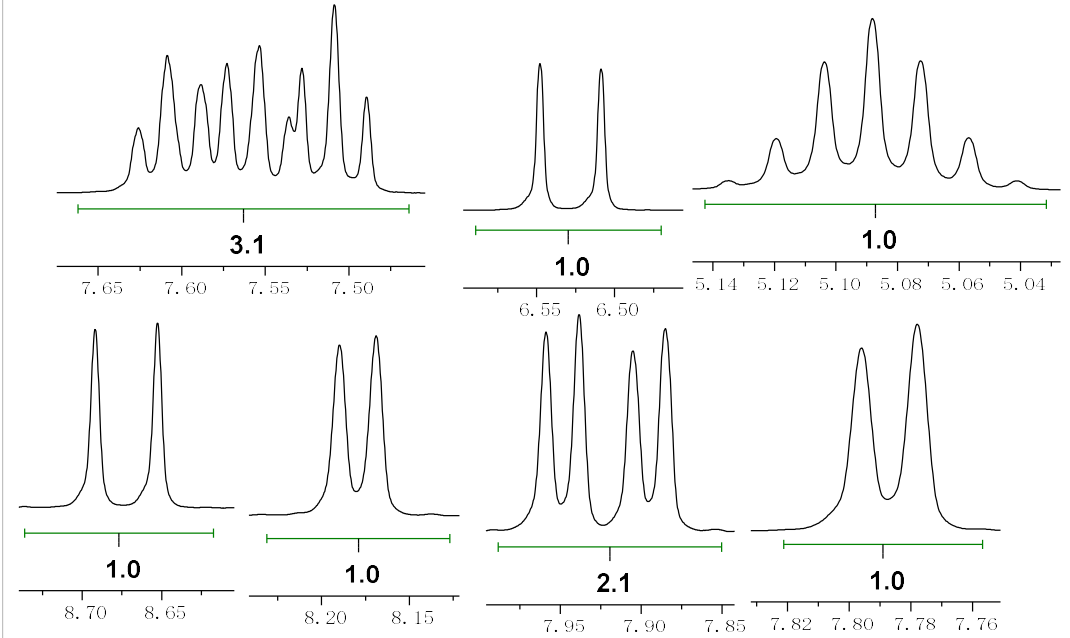

$400 \mathrm{MHz}, \mathrm{CDCl}_{3}{ }^{-1} \mathrm{H}$ NMR
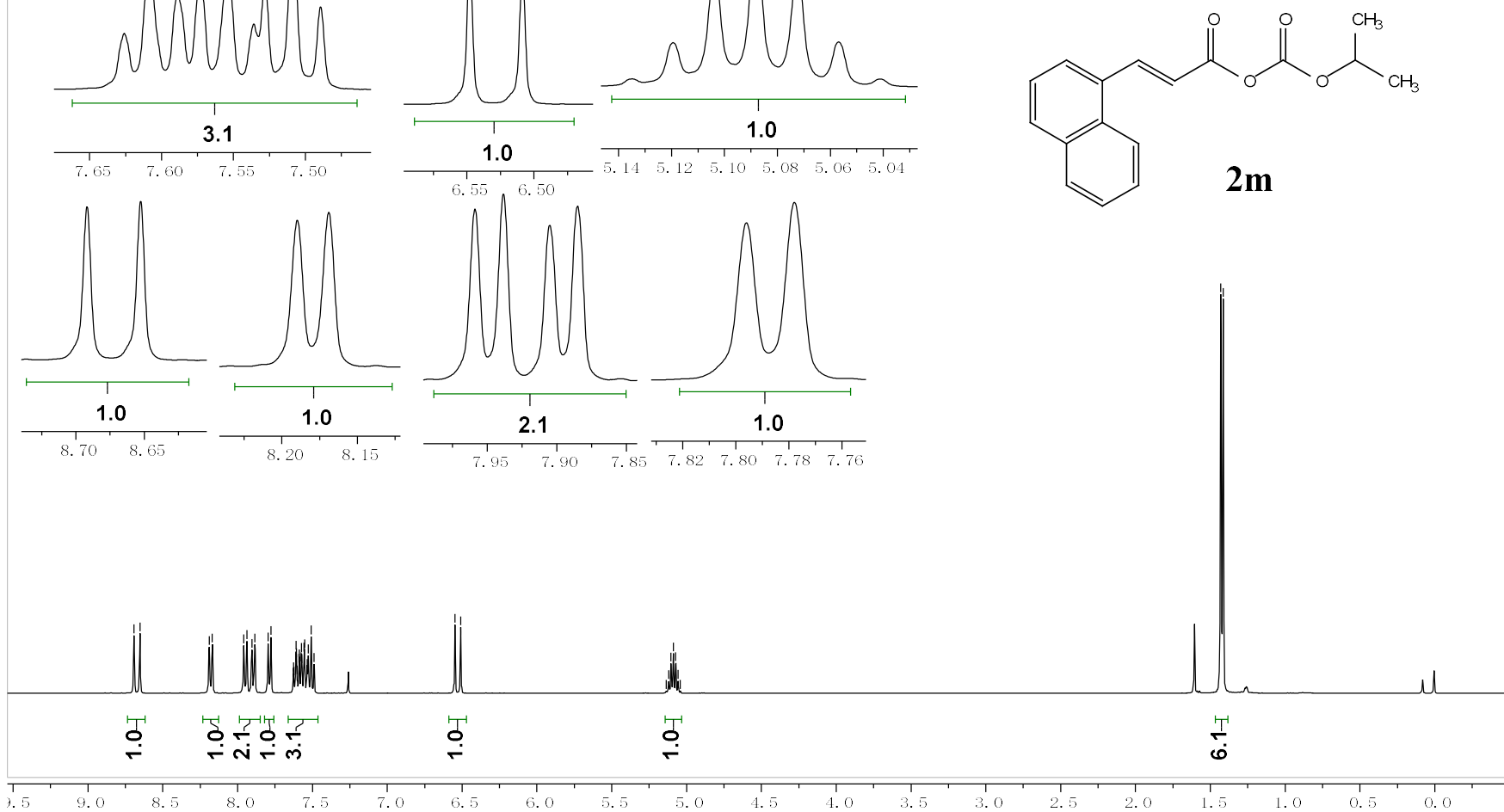


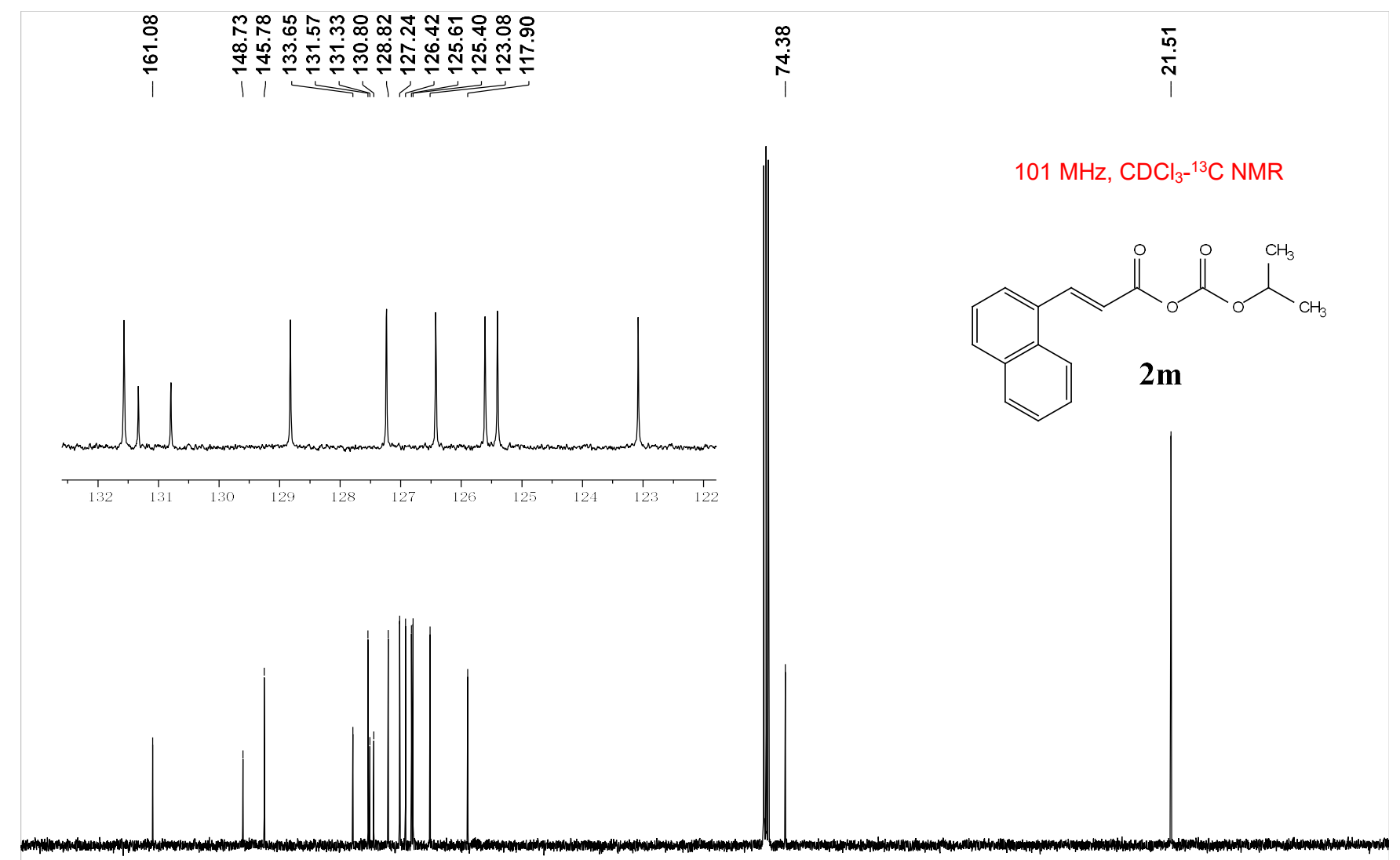




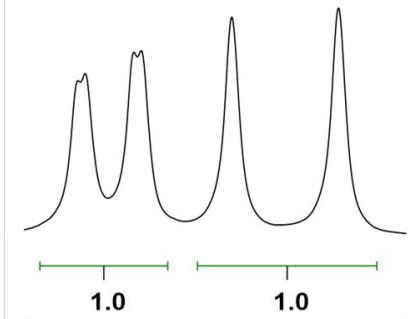

$\begin{array}{lllllllllll}7.76 & 7.74 & 7.72 & 7.70 & 7.68 & 7.66 & 7.64\end{array}$

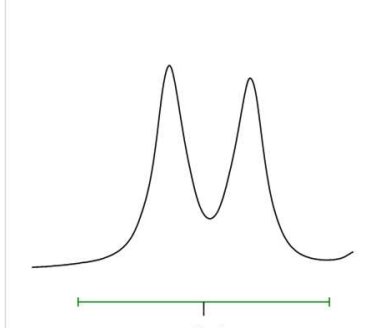

2.1
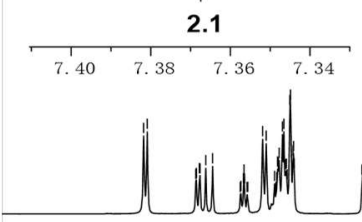

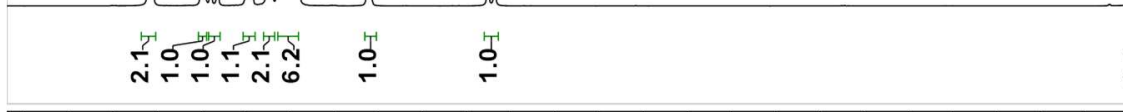
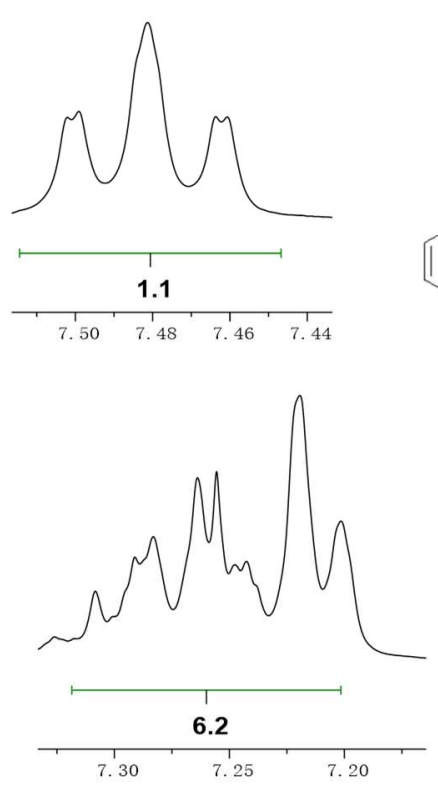

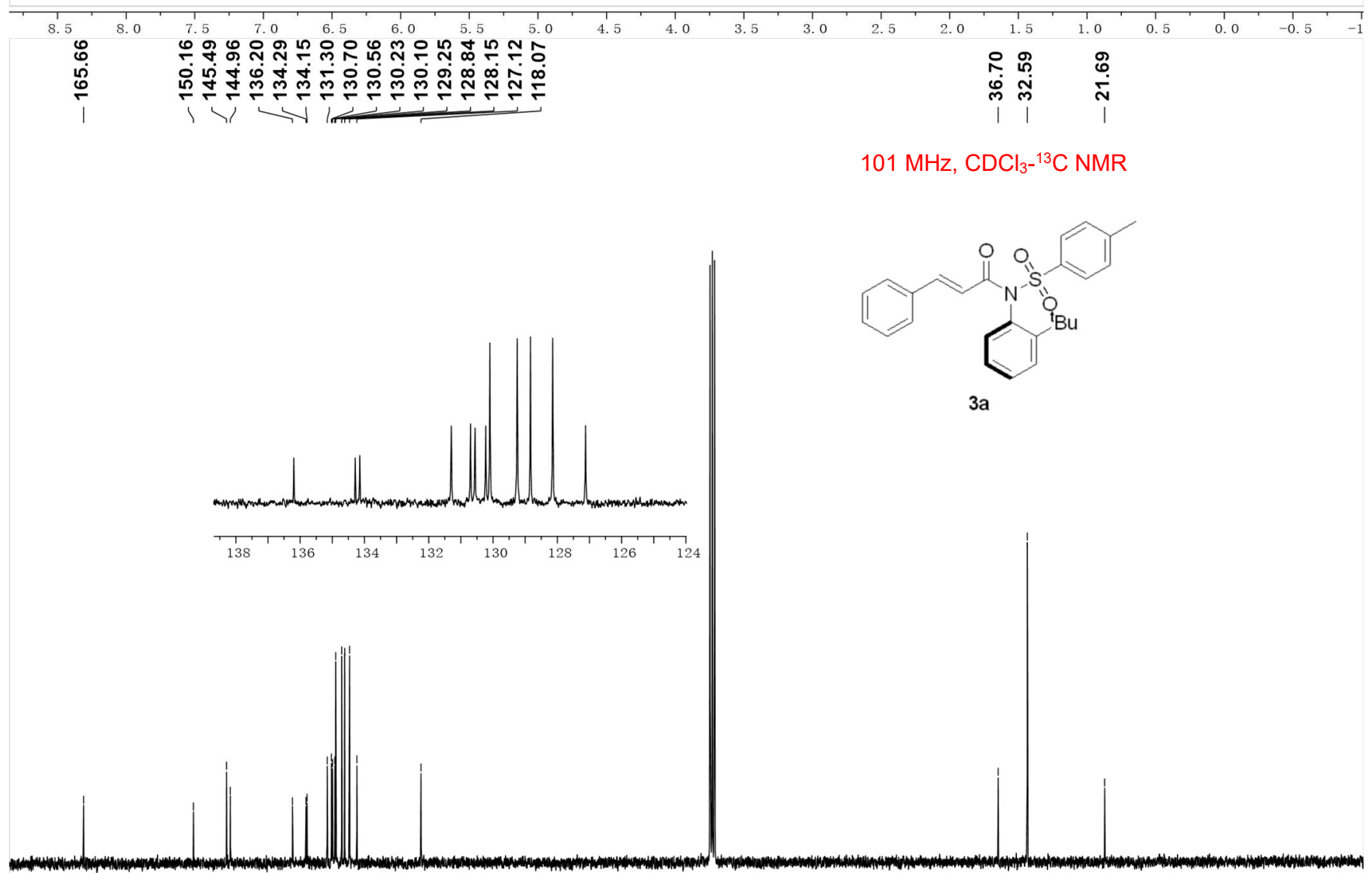

$400 \mathrm{MHz}, \mathrm{CDCl}_{3}-^{-1} \mathrm{H} \mathrm{NMR}$

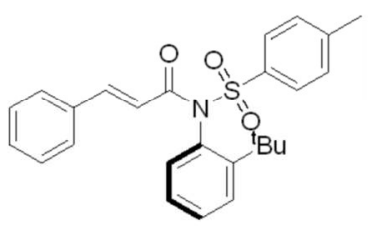

$3 a$

\section{管}

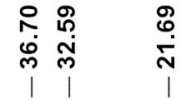

$101 \mathrm{MHz}, \mathrm{CDCl}_{3}{ }^{-13} \mathrm{C} \mathrm{NMR}$

3a

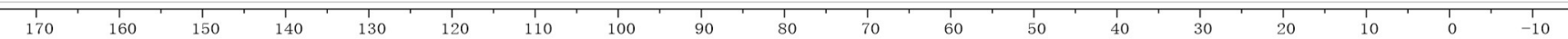




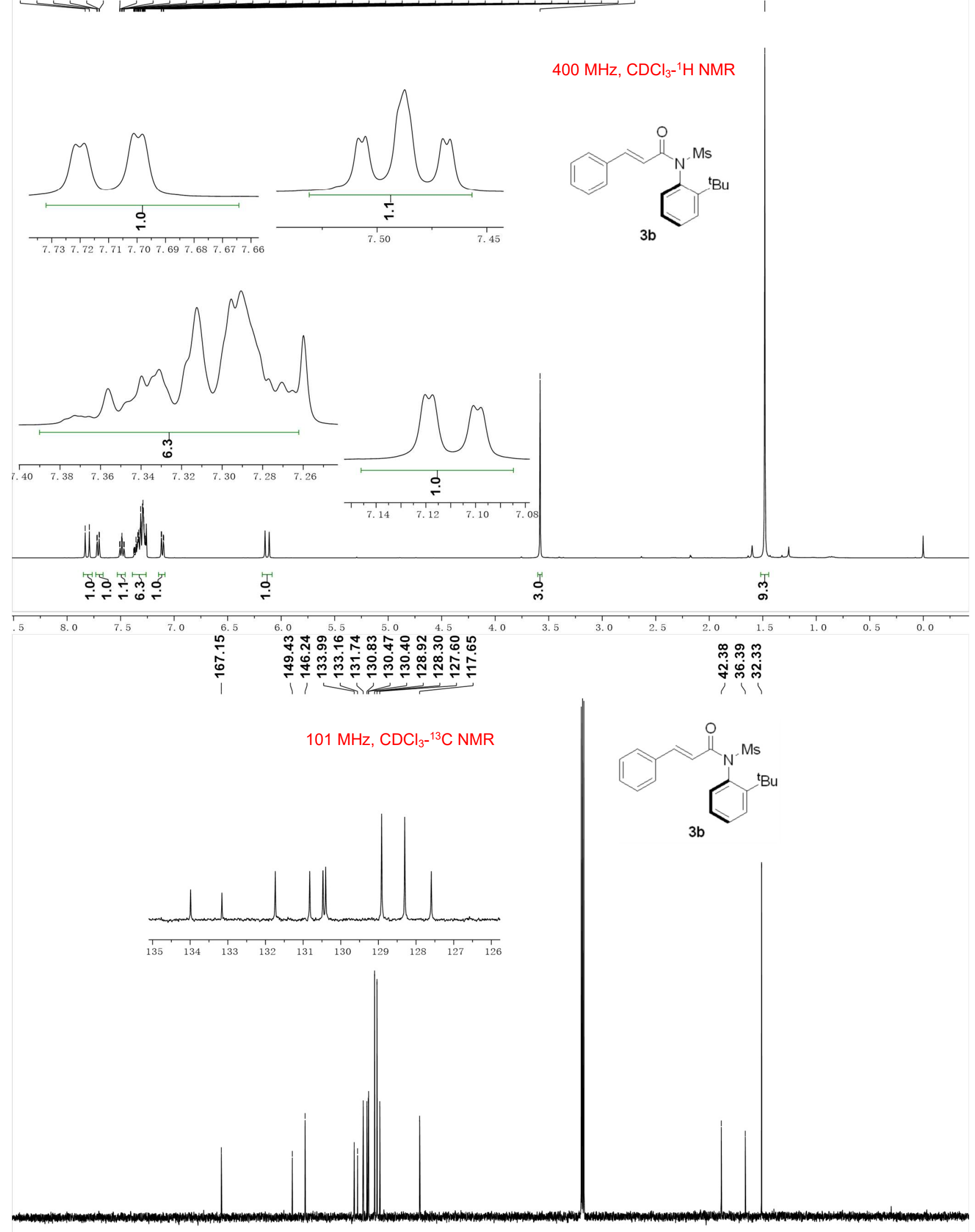

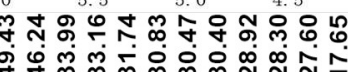

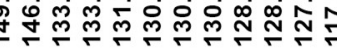

$101 \mathrm{MHz}, \mathrm{CDCl}_{3}-^{13} \mathrm{C} \mathrm{NMR}$
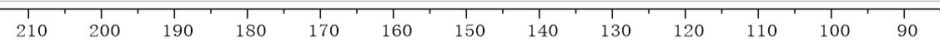

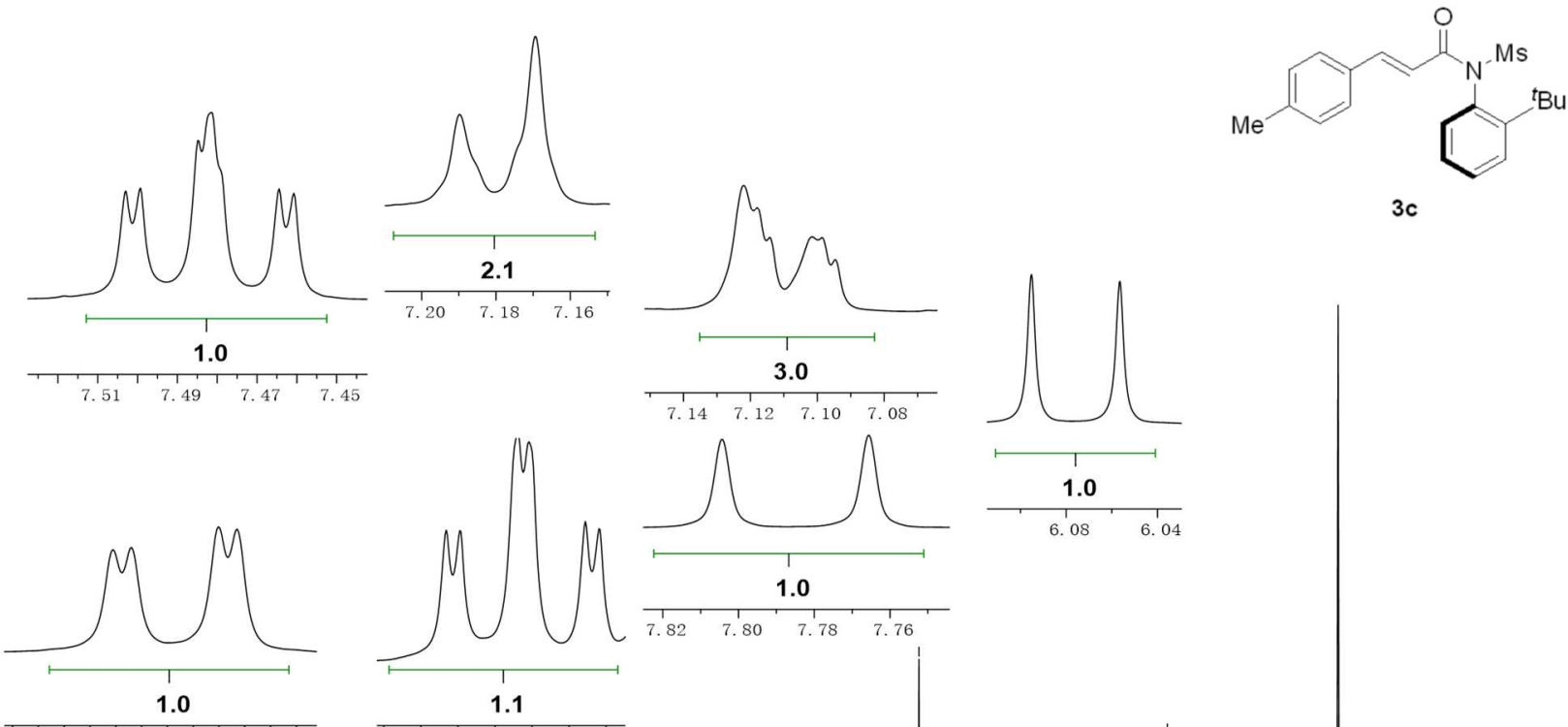

$\begin{array}{llllllll}7.73 & 7.72 & 7.71 & 7.70 & 7.69 & 7.68\end{array}$
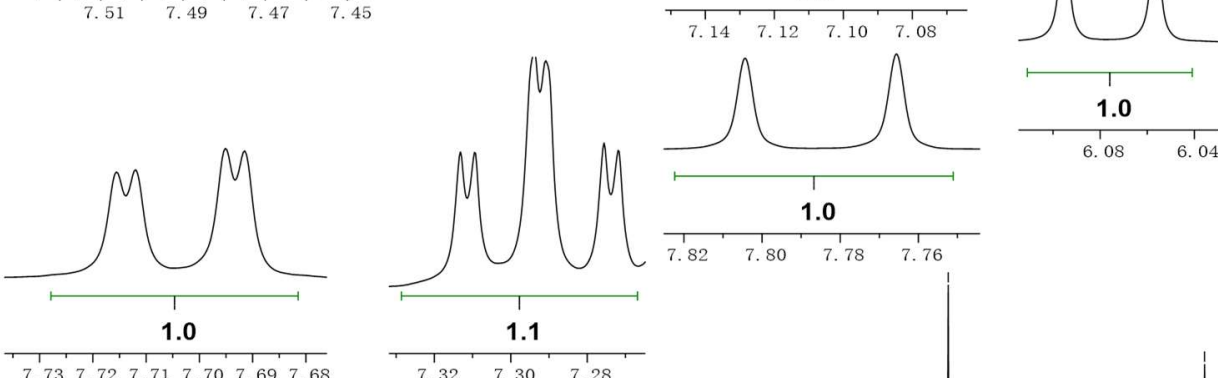

ii

$$
\text { 西 }
$$

itis whell

$\stackrel{4}{3}$

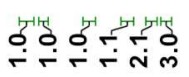

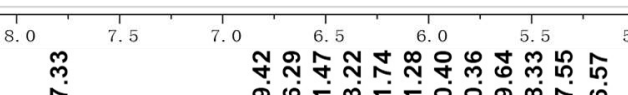

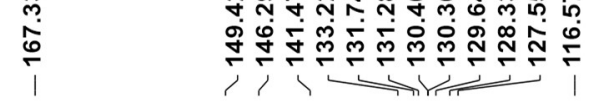

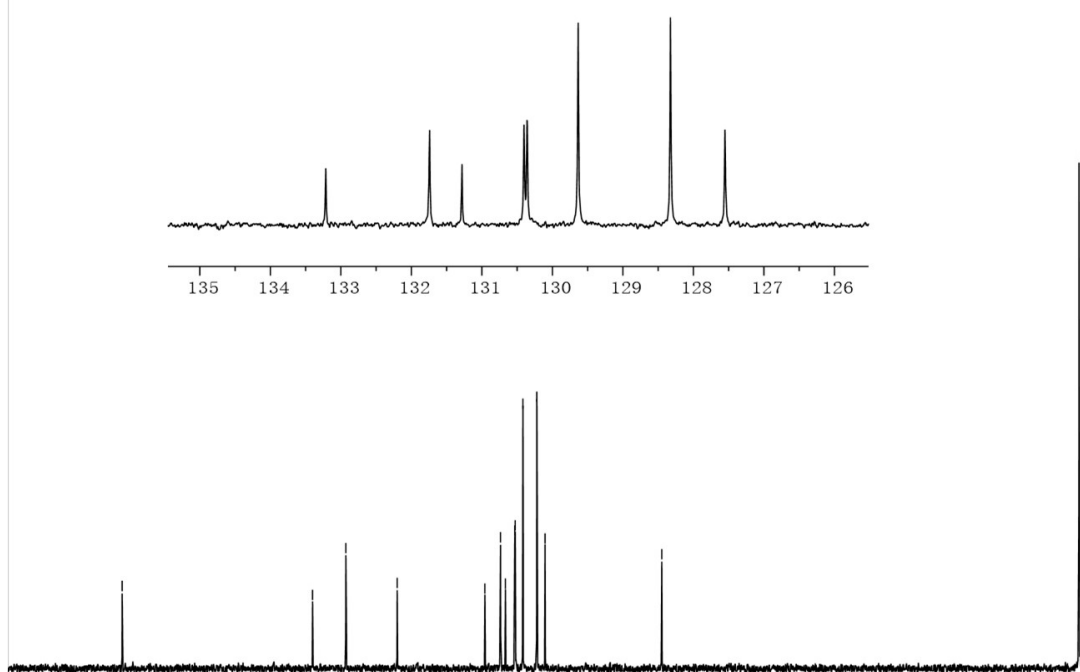

$101 \mathrm{MHz}, \mathrm{CDCl}_{3^{-}}{ }^{13} \mathrm{C} \mathrm{NMR}$

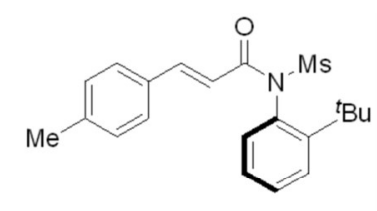

$3 c$

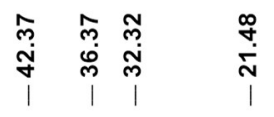

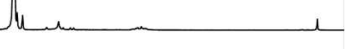
ळ 


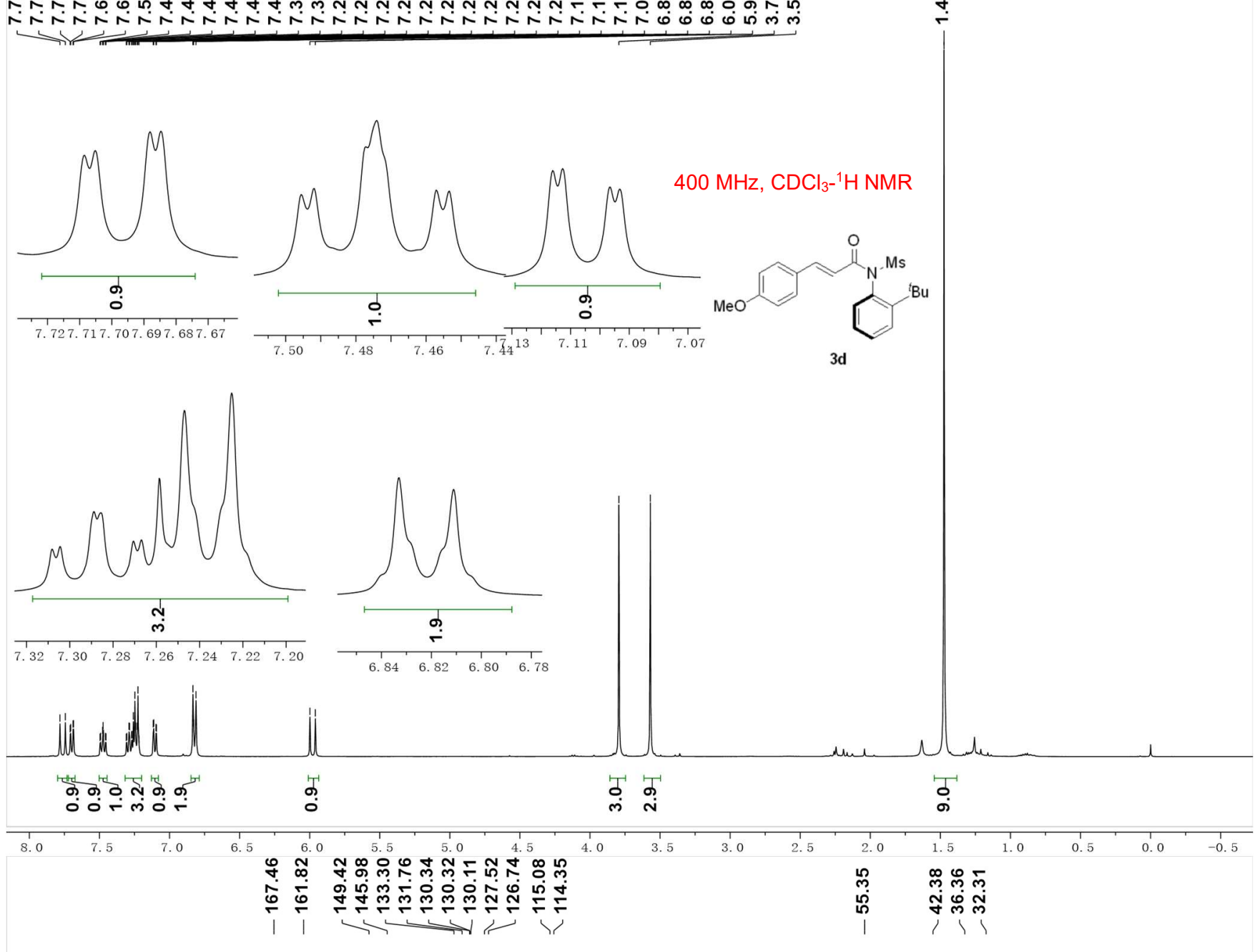

$101 \mathrm{MHz}, \mathrm{CDCl}_{3}-^{-13} \mathrm{C}$ NMR
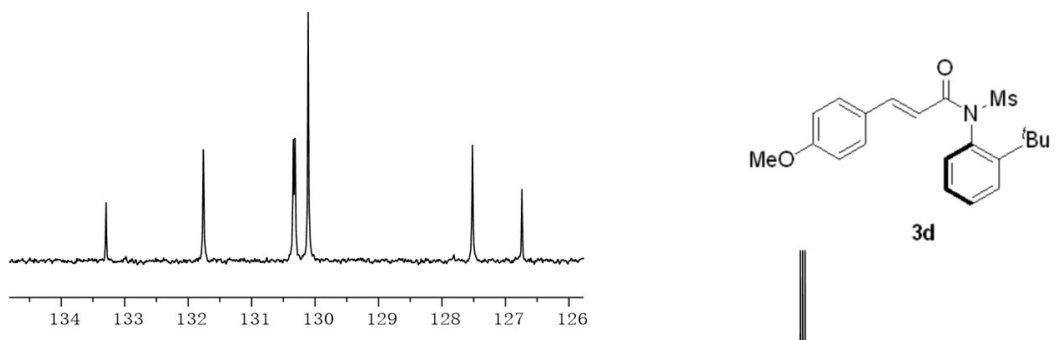

3d

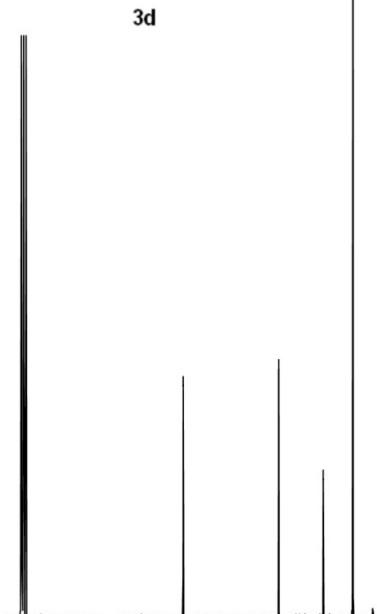



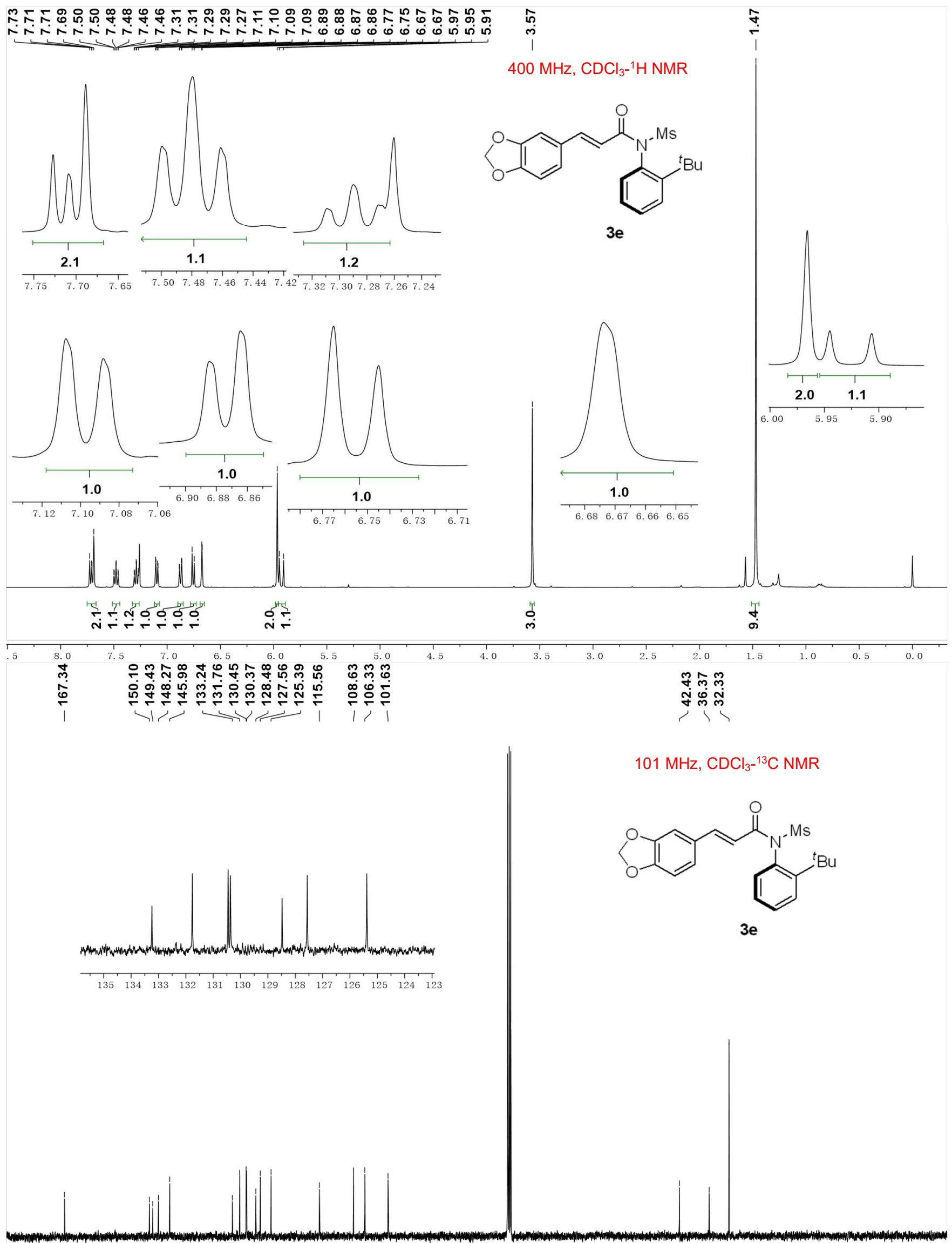


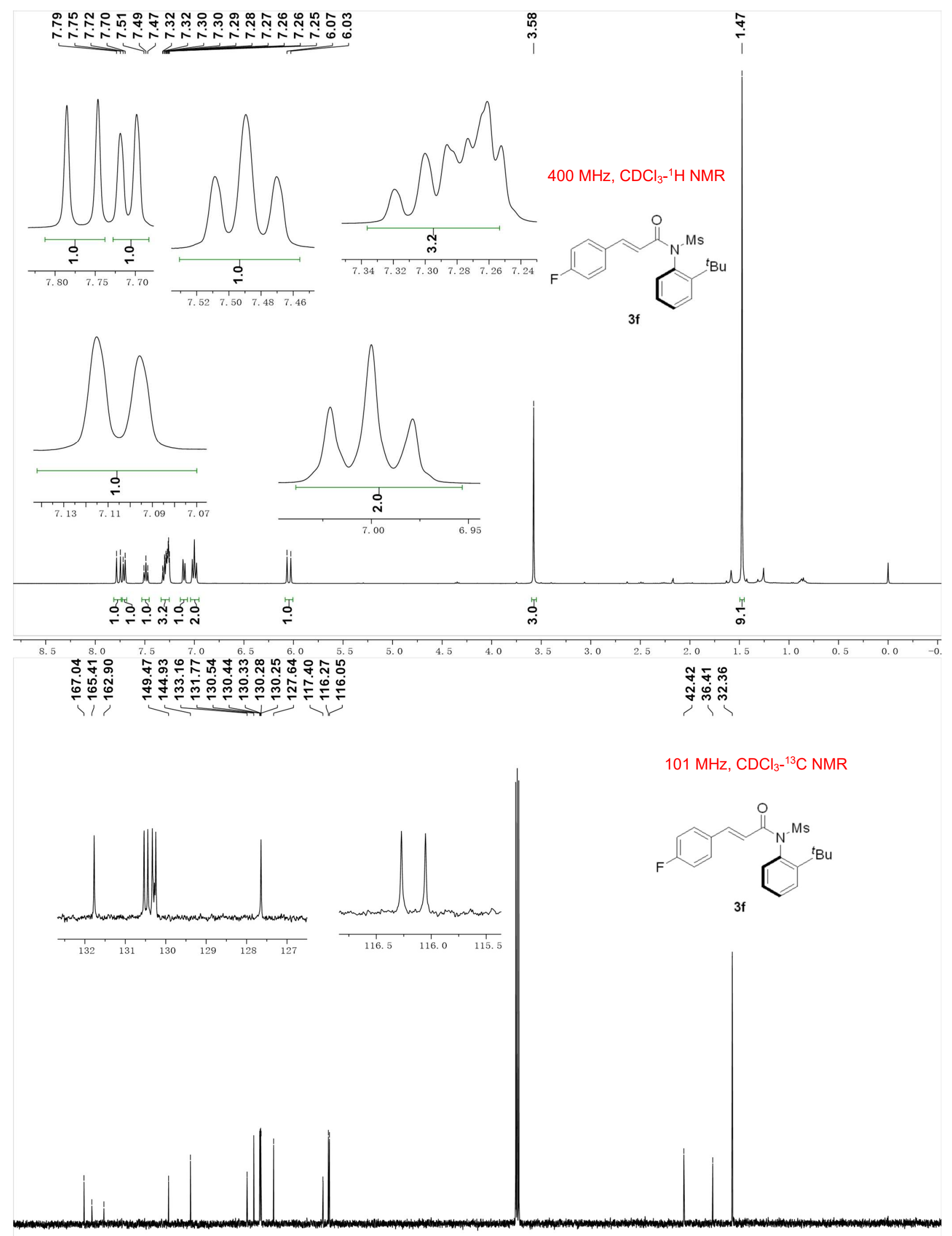



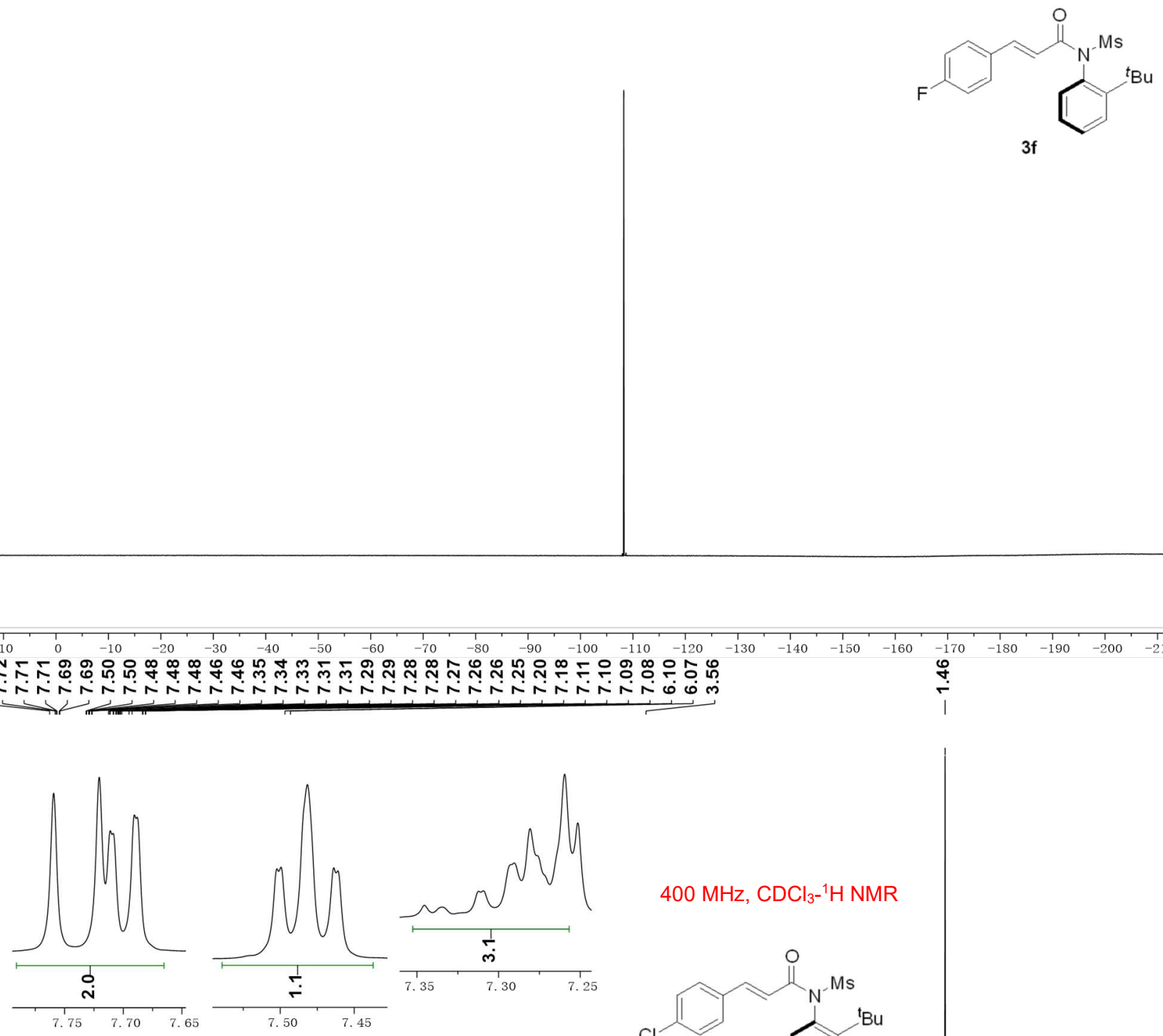

$400 \mathrm{MHz}, \mathrm{CDCl}_{3}-{ }^{1} \mathrm{H}$ NMR
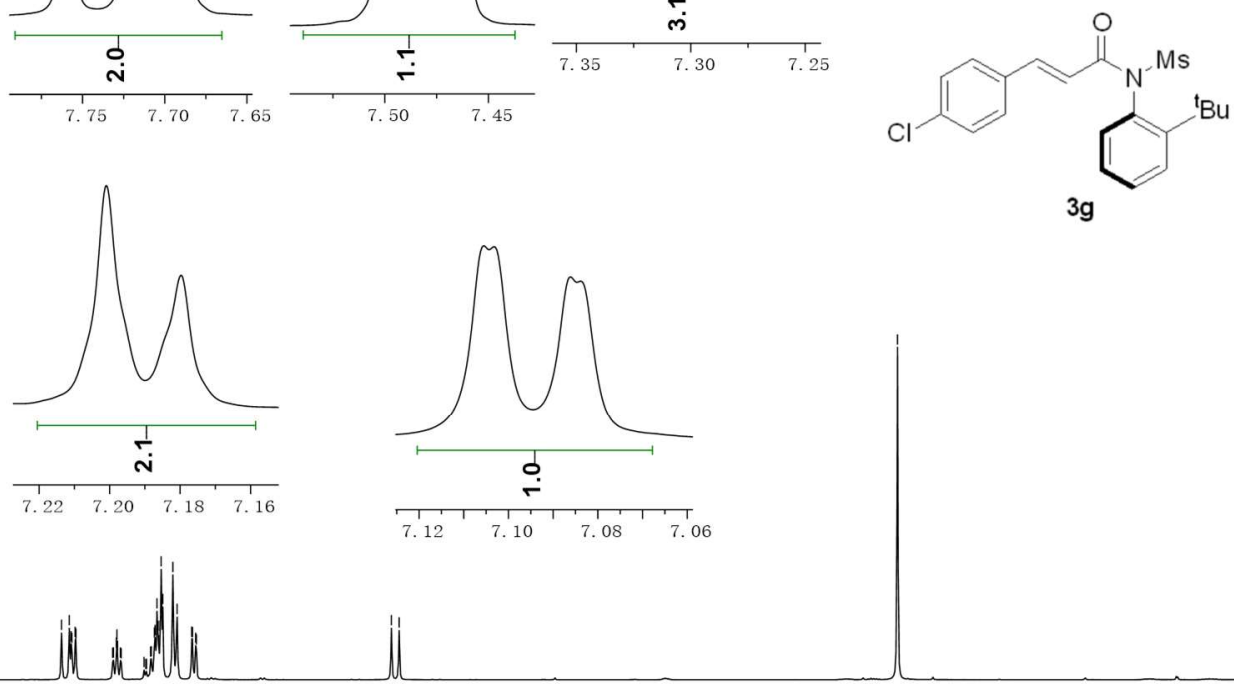

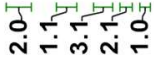

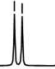

离

崔 

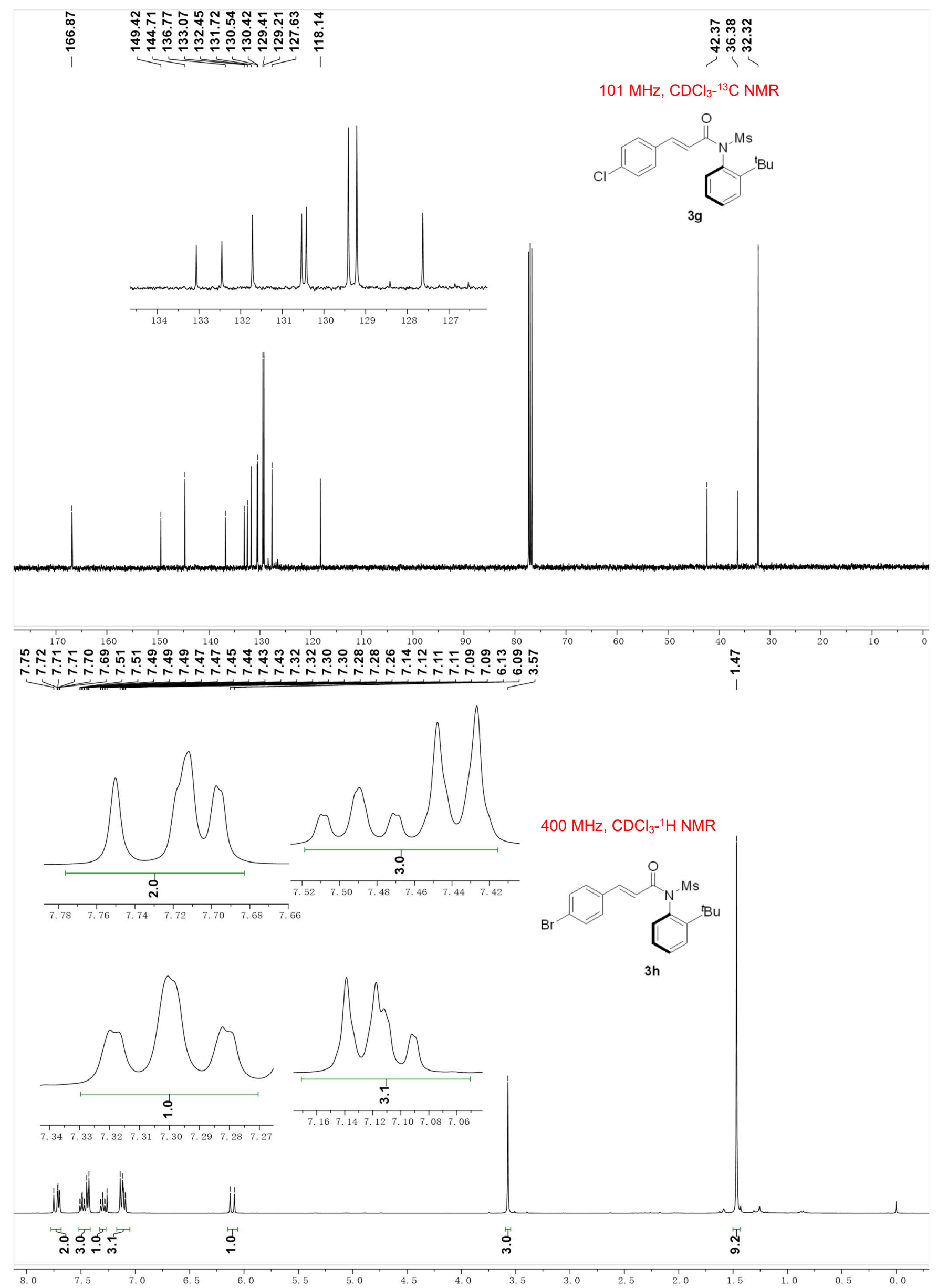


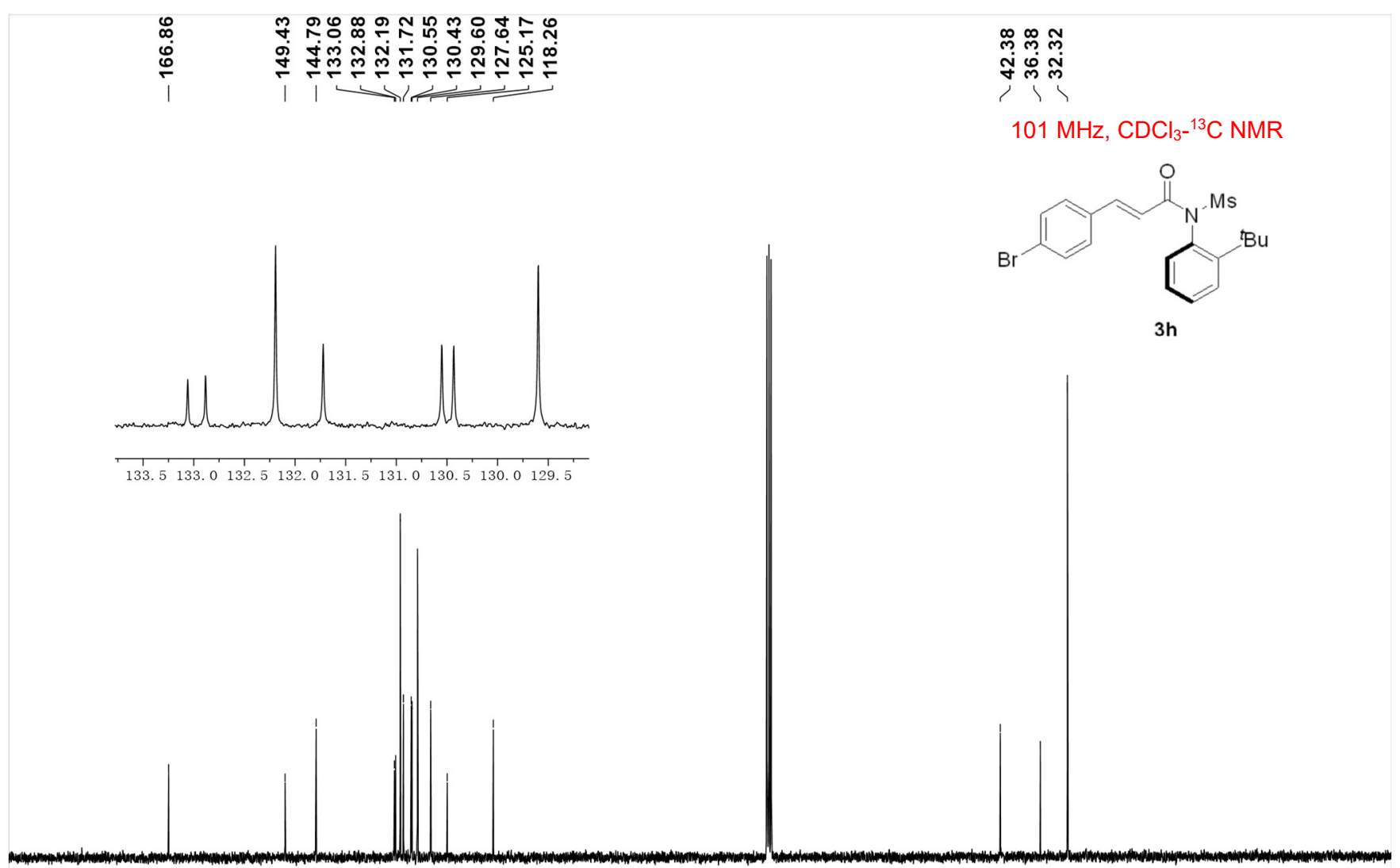

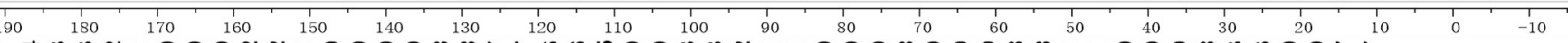

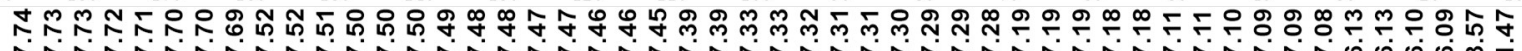

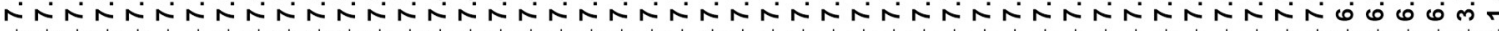
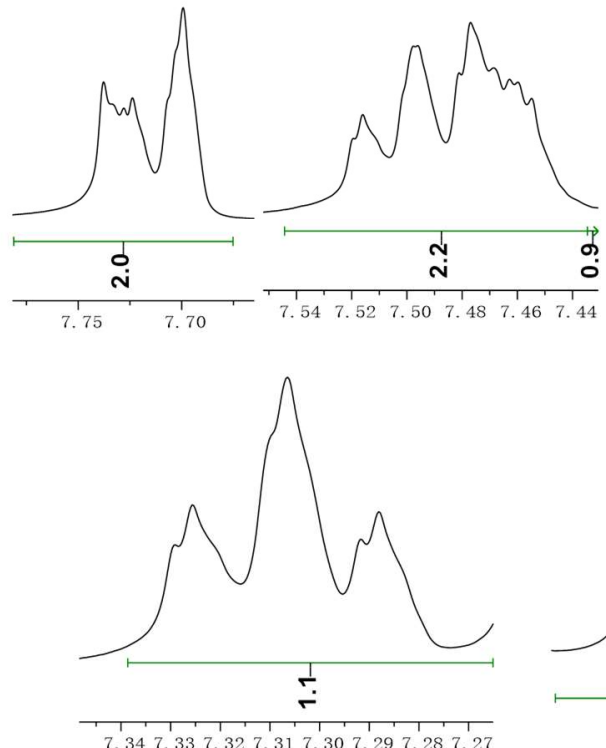

N
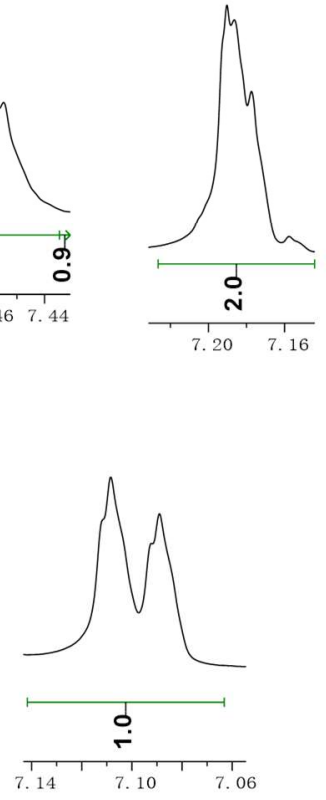

$400 \mathrm{MHz}, \mathrm{CDCl}_{3}{ }^{-1} \mathrm{H} \mathrm{NMR}$

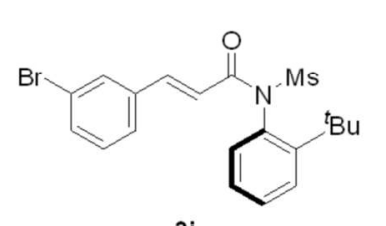

$3 \mathbf{i}$

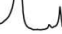

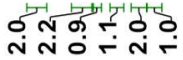

寄

憼

每 

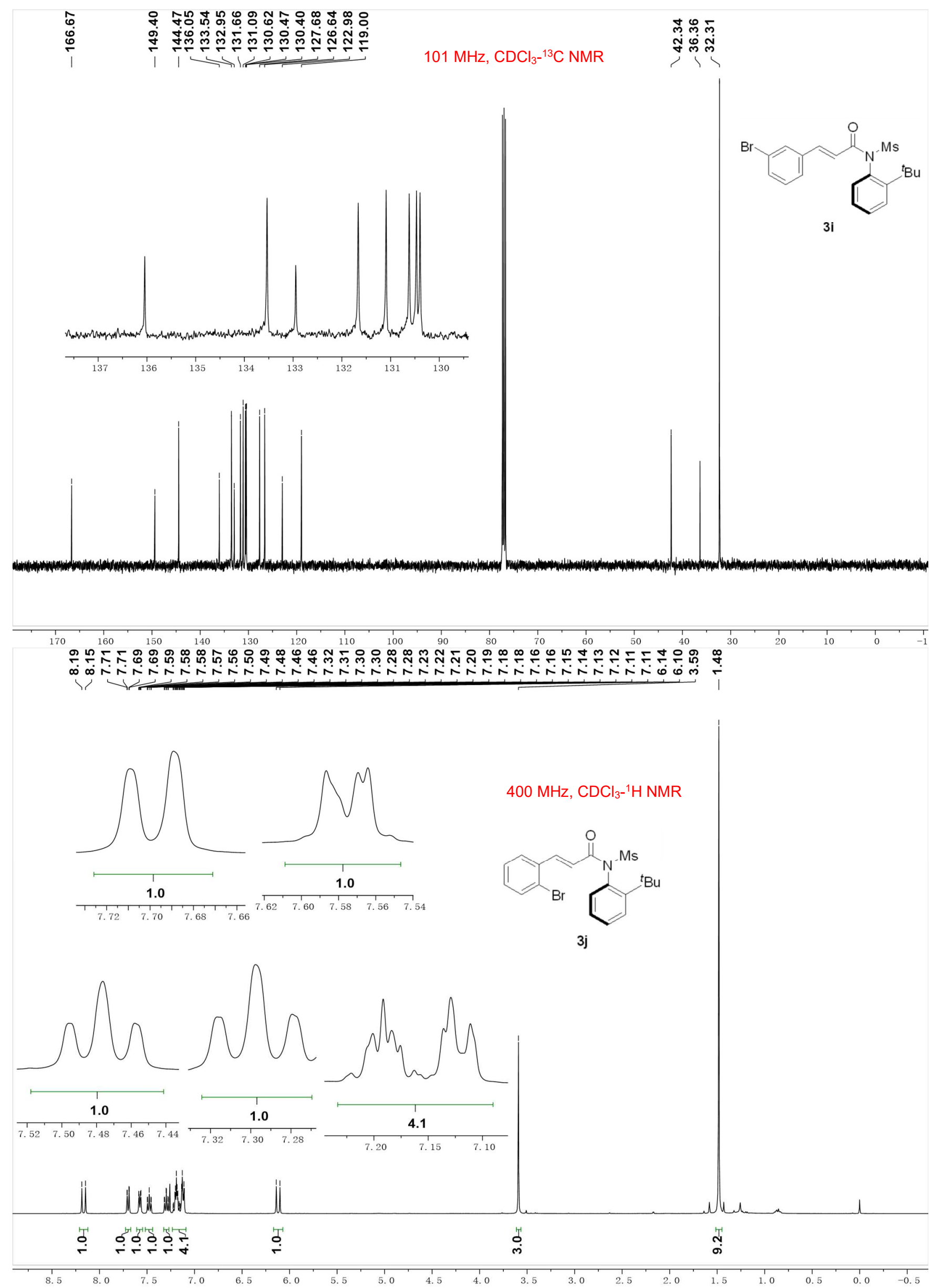


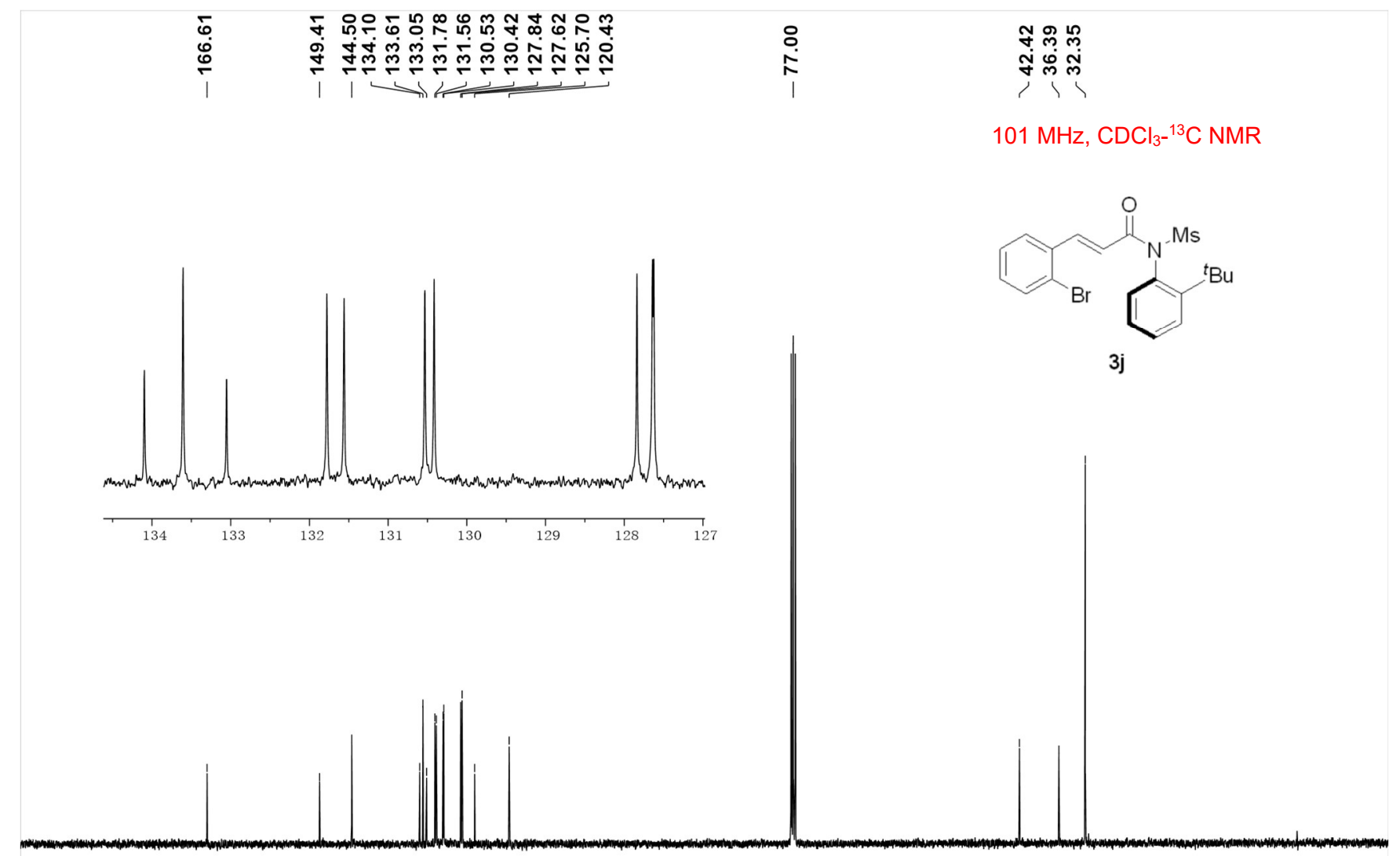

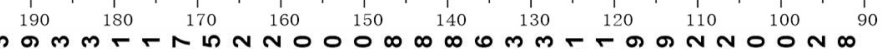

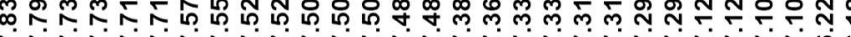

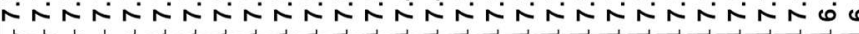
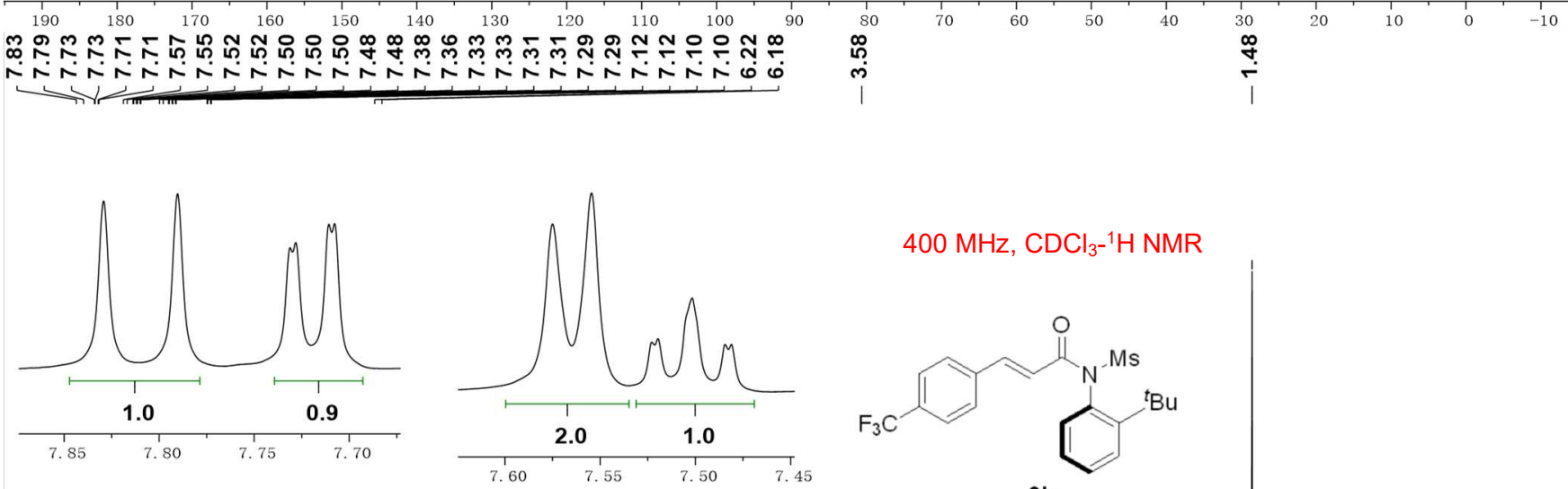

$400 \mathrm{MHz}, \mathrm{CDCl}_{3}-{ }^{1} \mathrm{H}$ NMR
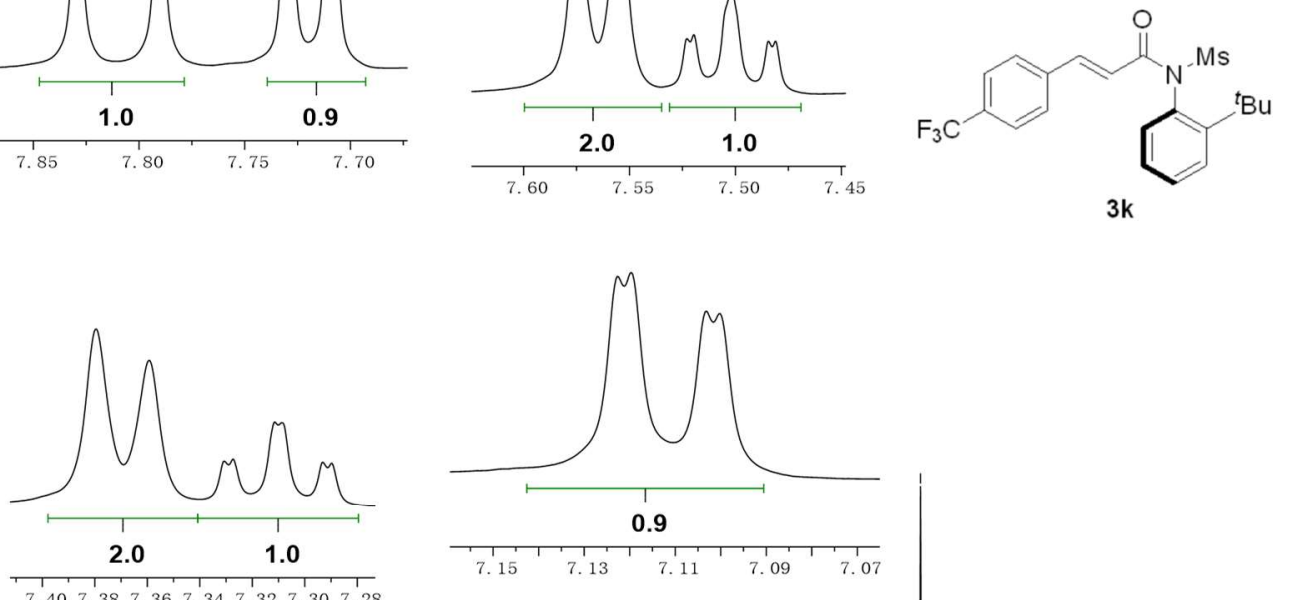

$3 k$
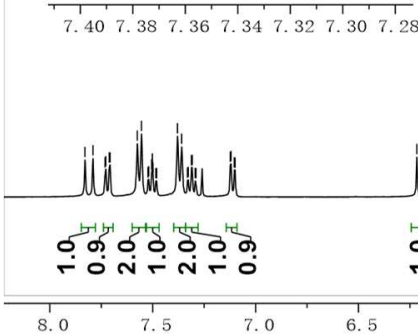

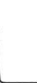

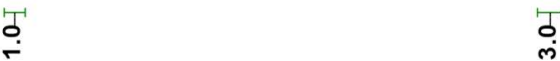

웅

क.

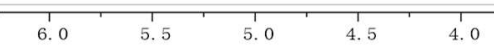




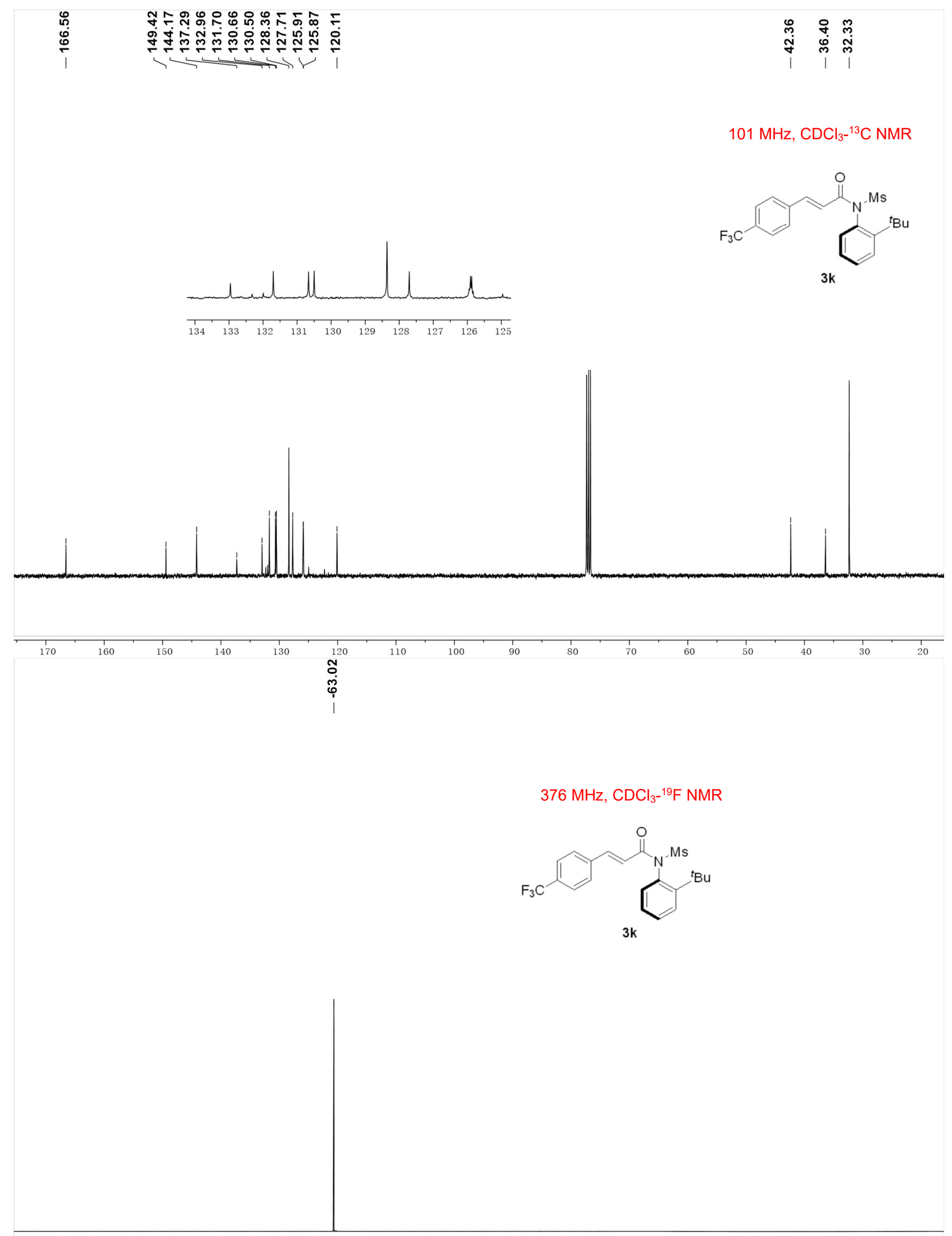

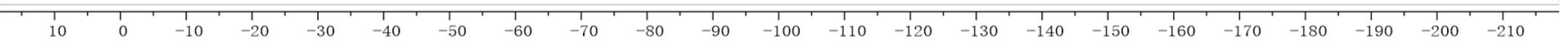



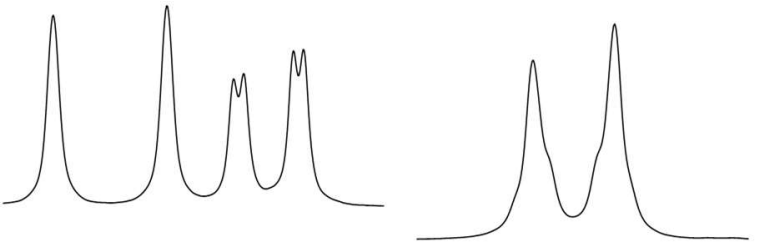

$400 \mathrm{MHz}, \mathrm{CDCl}_{3}-^{-1} \mathrm{H} \mathrm{NMR}$
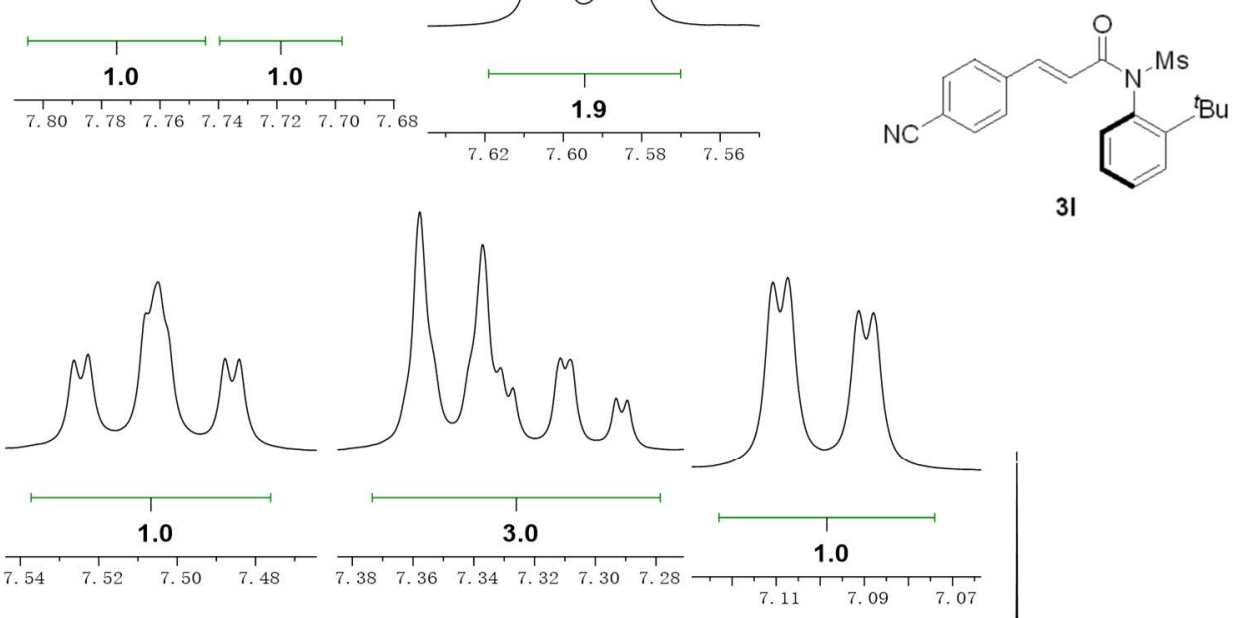

ivilubilit

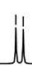

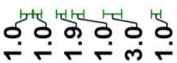

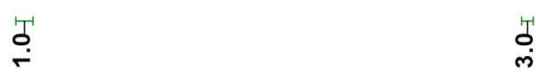

菑

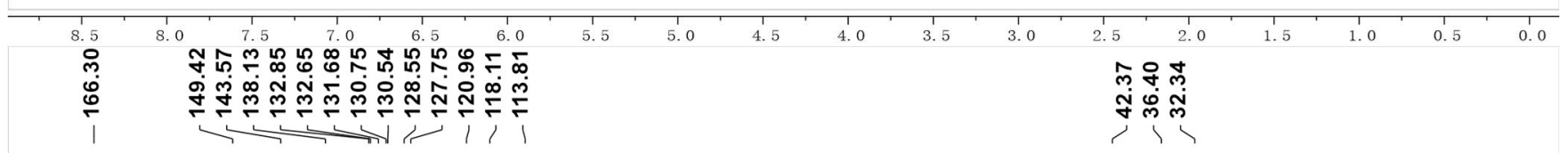

$101 \mathrm{MHz}, \mathrm{CDCl}_{3}-^{13} \mathrm{C}$ NMR
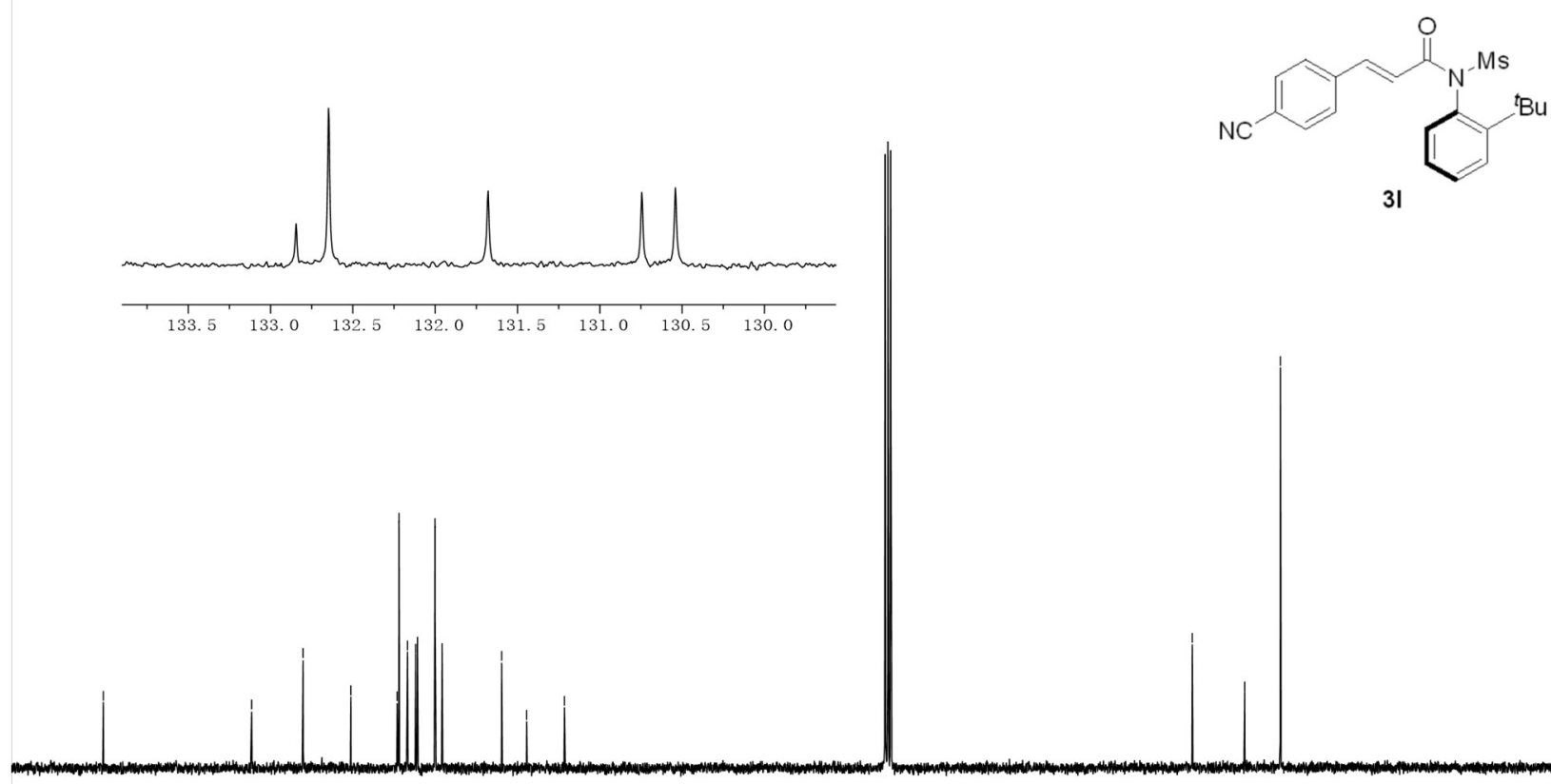

31

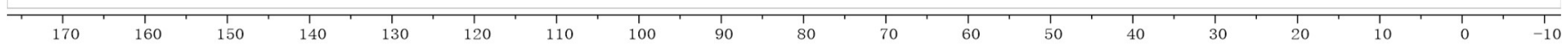




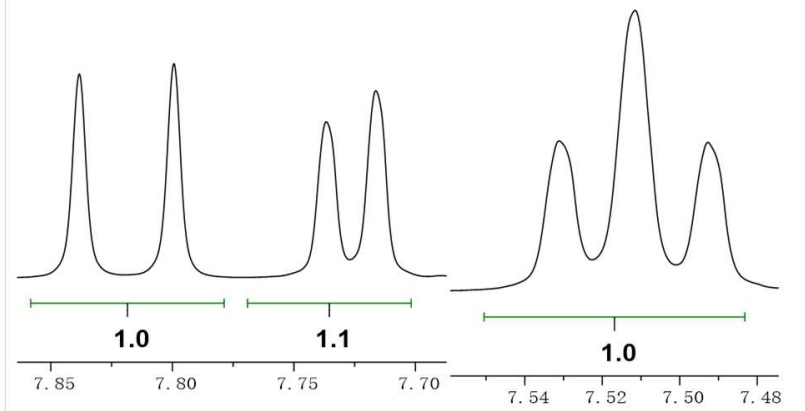

$400 \mathrm{MHz}, \mathrm{CDCl}_{3}{ }^{-1} \mathrm{H} \mathrm{NMR}$<smiles>CN(C(=O)/C=C/c1ccc([N+](=O)[O-])cc1)c1ccccc1C(C)(C)C</smiles>
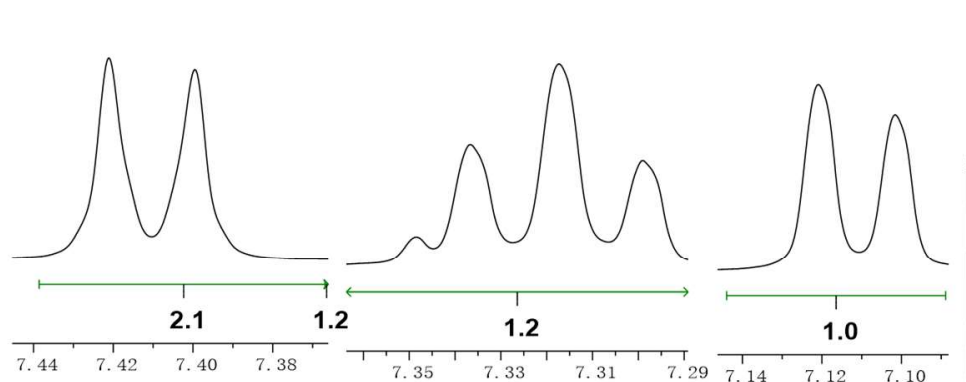

1.0
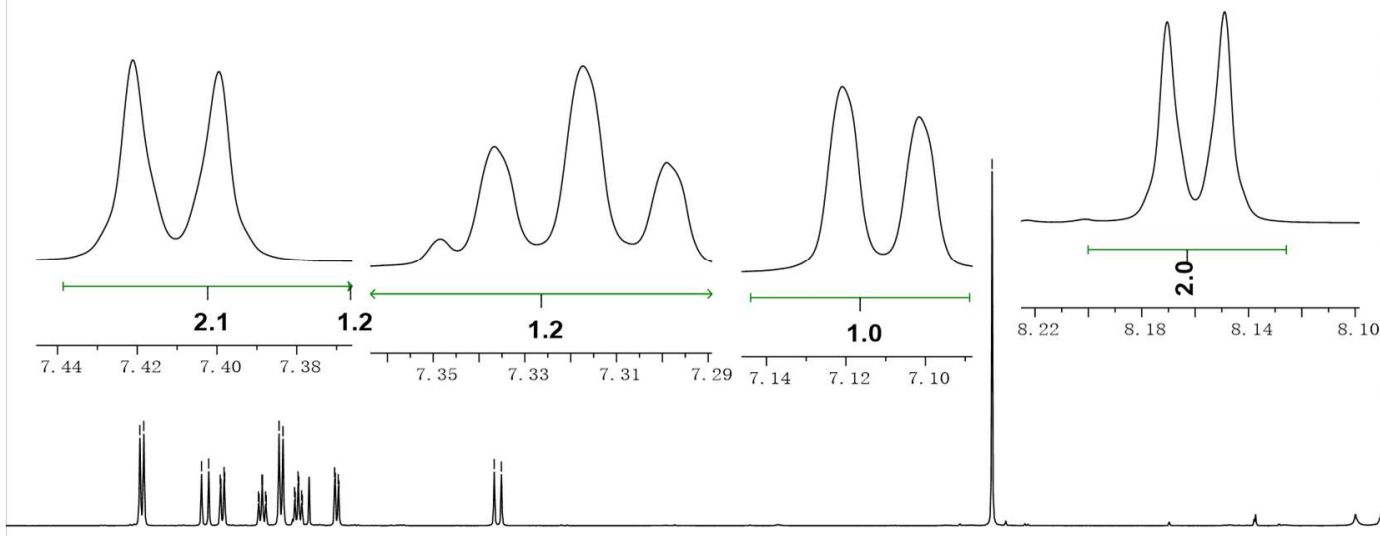

u
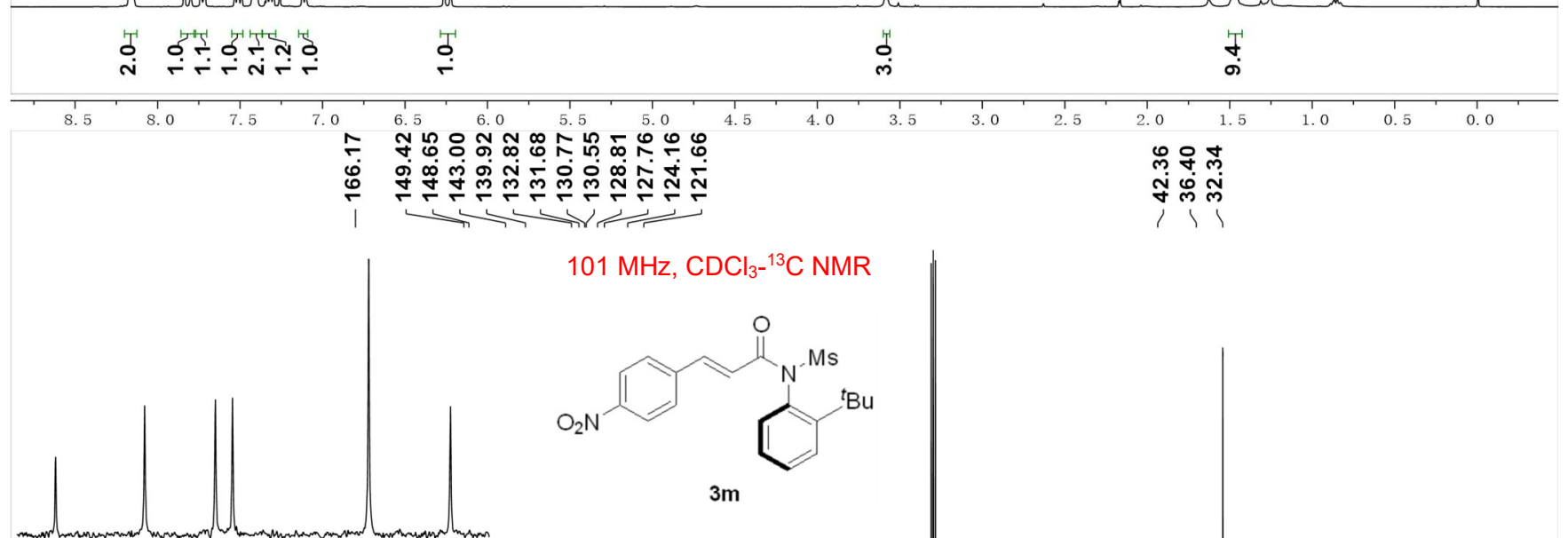

$101 \mathrm{MHz}, \mathrm{CDCl}_{3}{ }^{-13} \mathrm{C} \mathrm{NMR}$
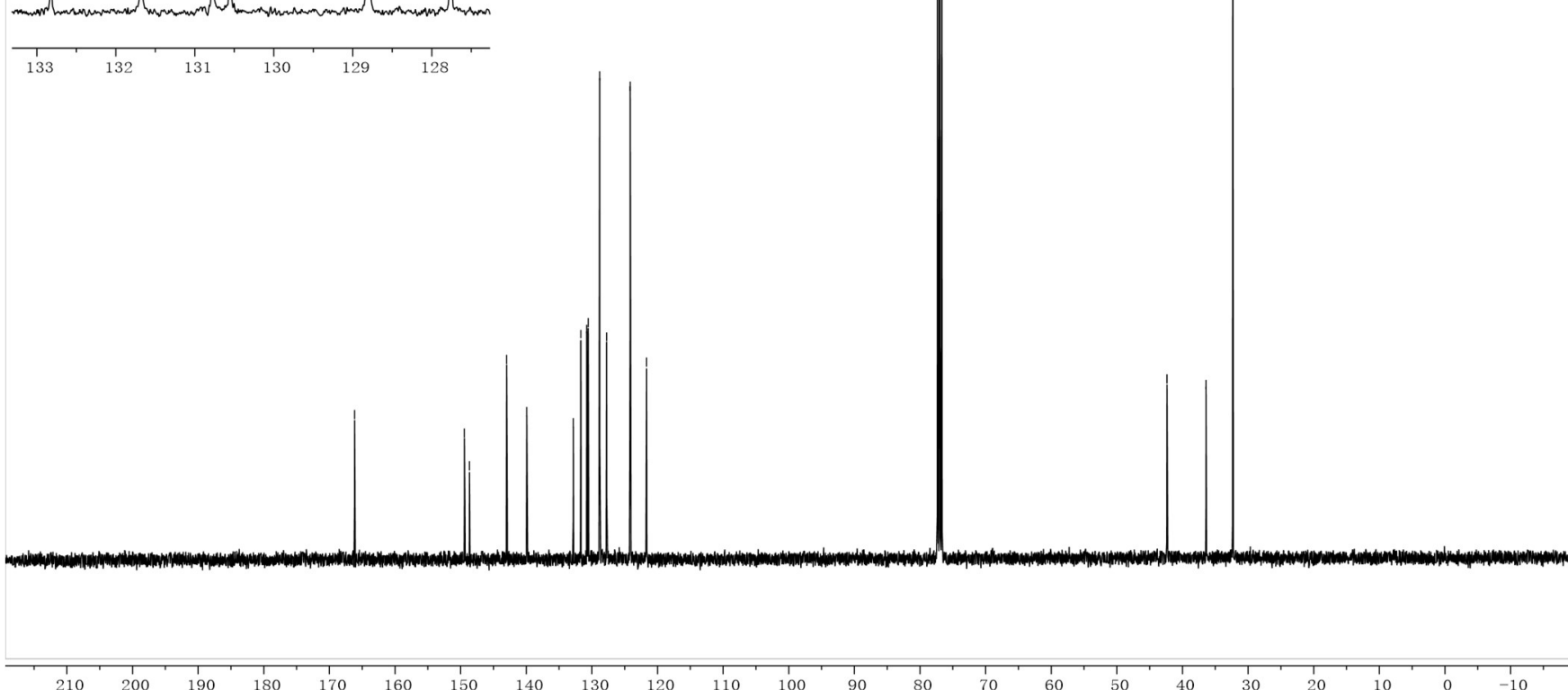


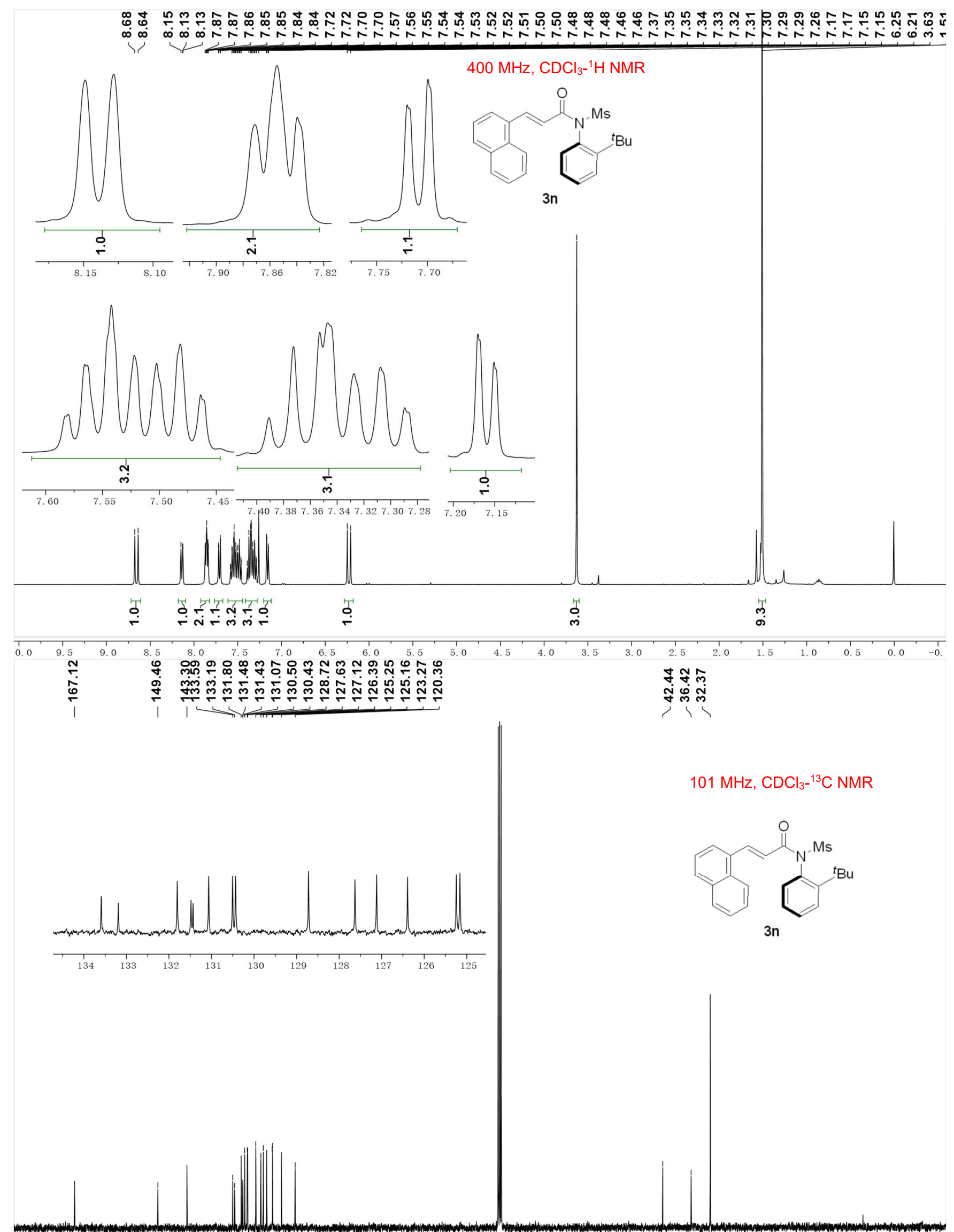



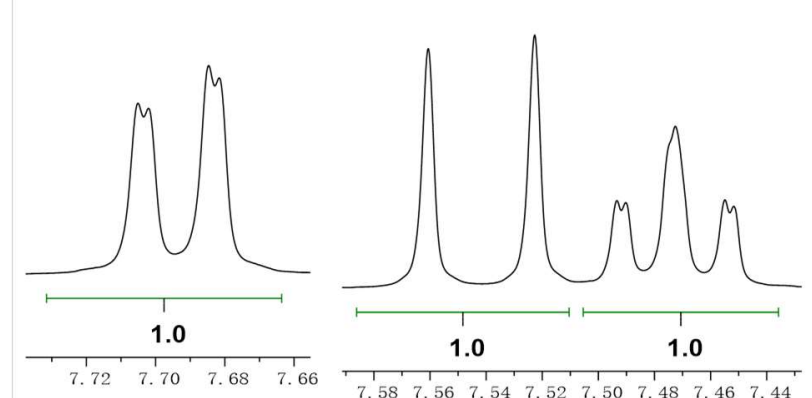

$400 \mathrm{MHz}, \mathrm{CDCl}_{3}-^{-1} \mathrm{H} \mathrm{NMR}$
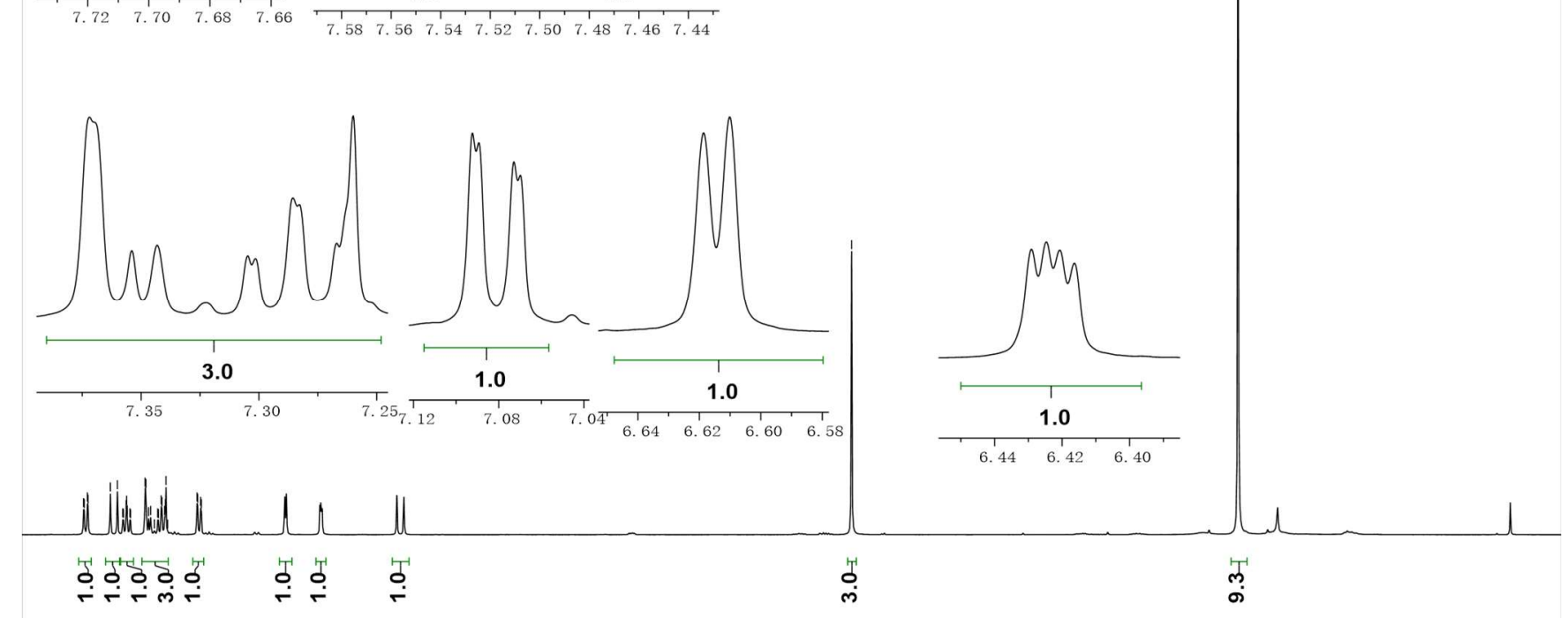

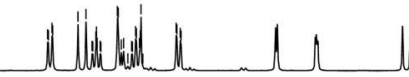

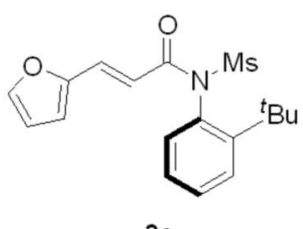

30

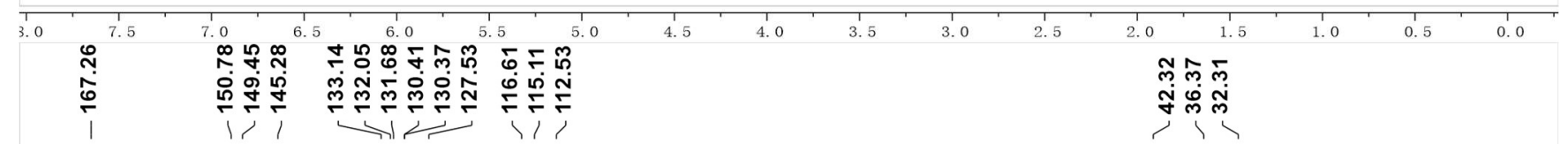

$101 \mathrm{MHz}, \mathrm{CDCl}_{3}-{ }^{13} \mathrm{C} \mathrm{NMR}$

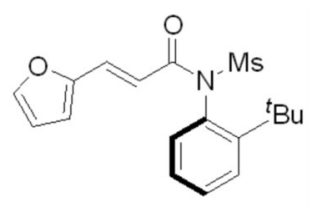

30

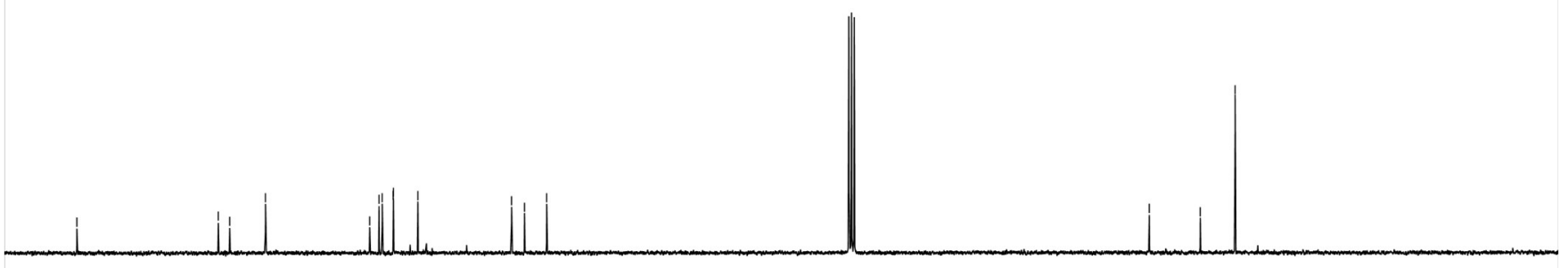




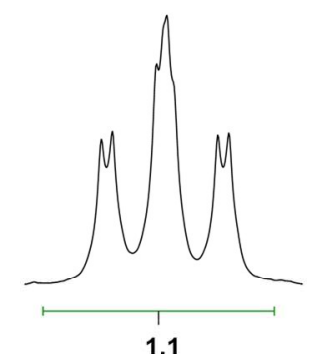

$\begin{array}{cccccc}7.52 & 7.50 & 7.48 & 7.46 & 7.44\end{array}$

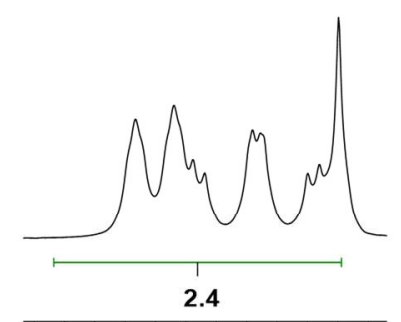

$\begin{array}{llllllll}7.36 & 7.34 & 7.32 & 7.30 & 7.28 & 7.26\end{array}$
$400 \mathrm{MHz}, \mathrm{CDCl}_{3}{ }^{-1} \mathrm{H}$ NMR

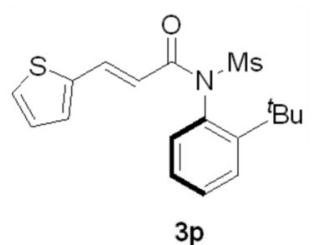

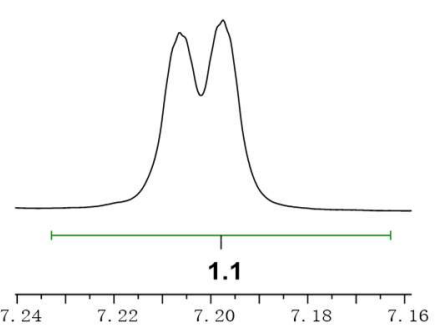

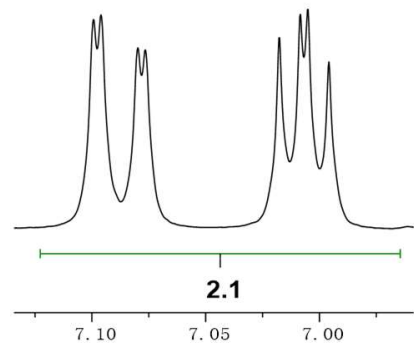

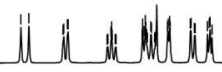
$\|$

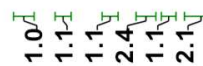
$\stackrel{+}{\stackrel{2}{*}}$ $\stackrel{\leftrightarrow}{\circ}$ 管

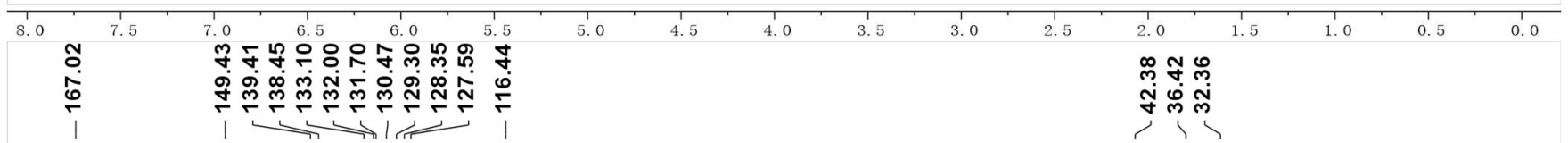

$101 \mathrm{MHz}, \mathrm{CDCl}_{3}{ }^{-13} \mathrm{C}$ NMR
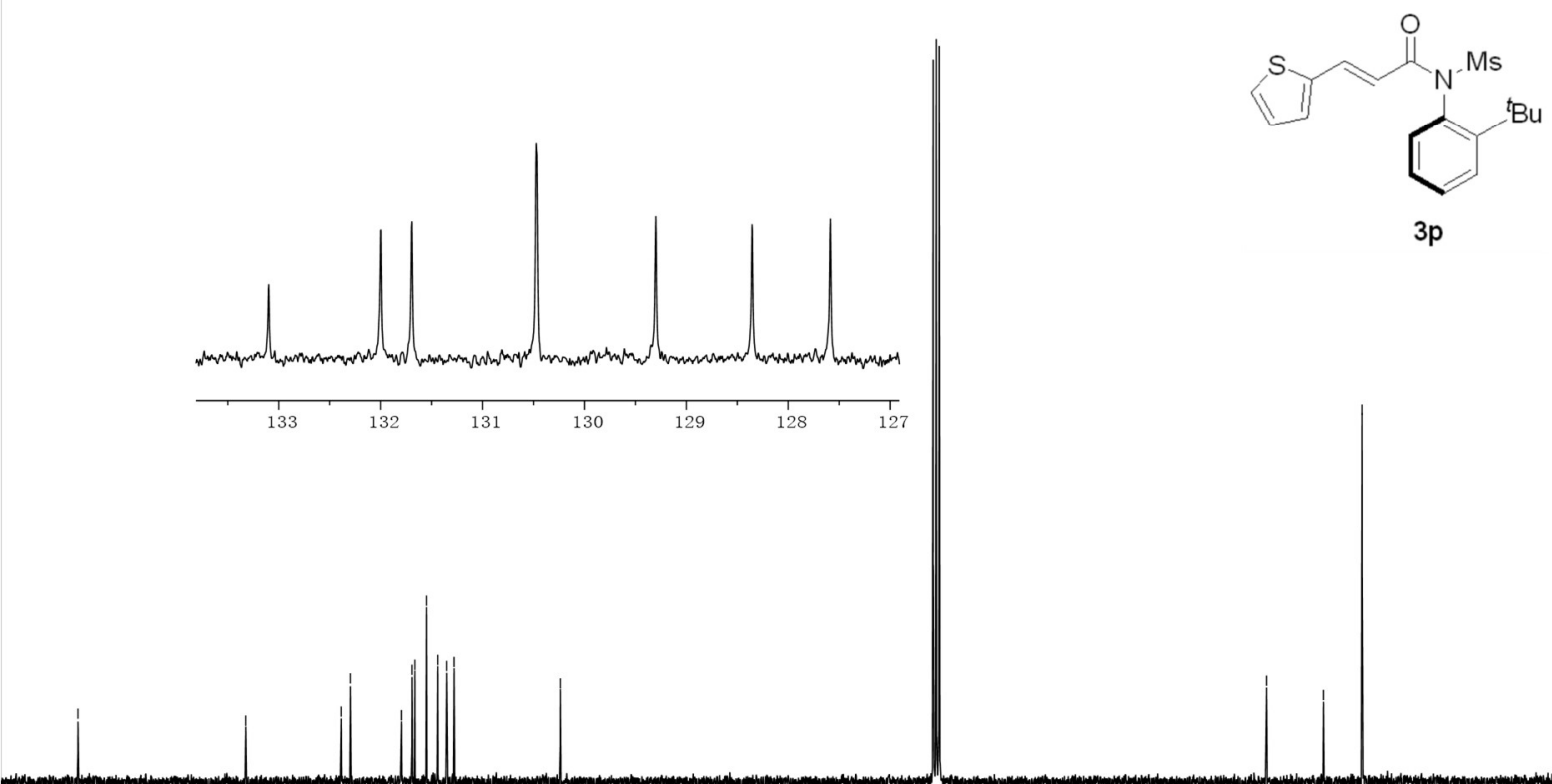

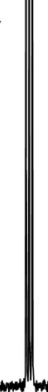




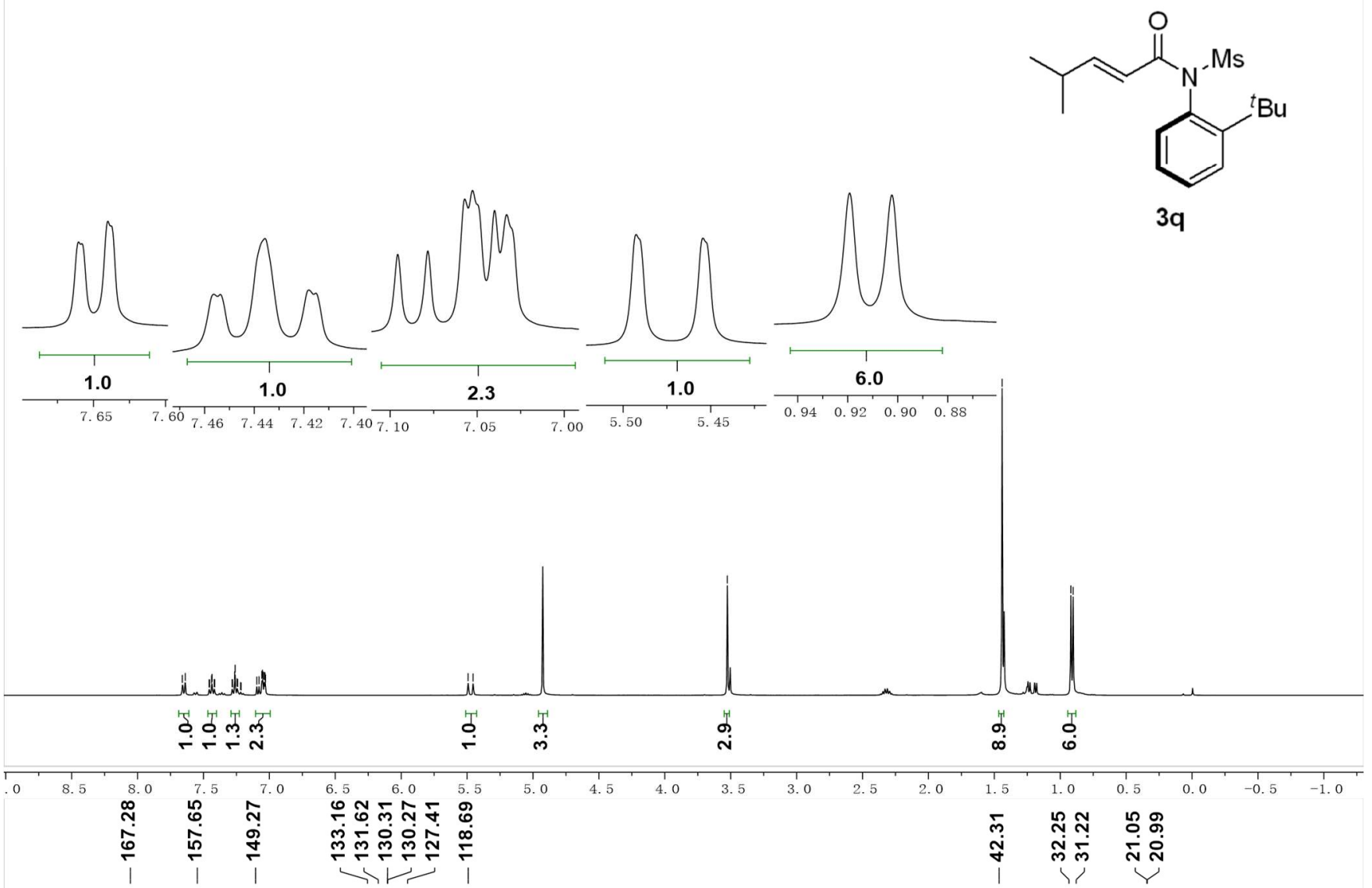

$101 \mathrm{MHz}, \mathrm{CDCl}_{3} \mathbf{-}^{13} \mathrm{C} \mathrm{NMR}$<smiles>CC(C)/C=C/C(=O)N(C)c1ccccc1C(C)(C)C</smiles>

$3 q$

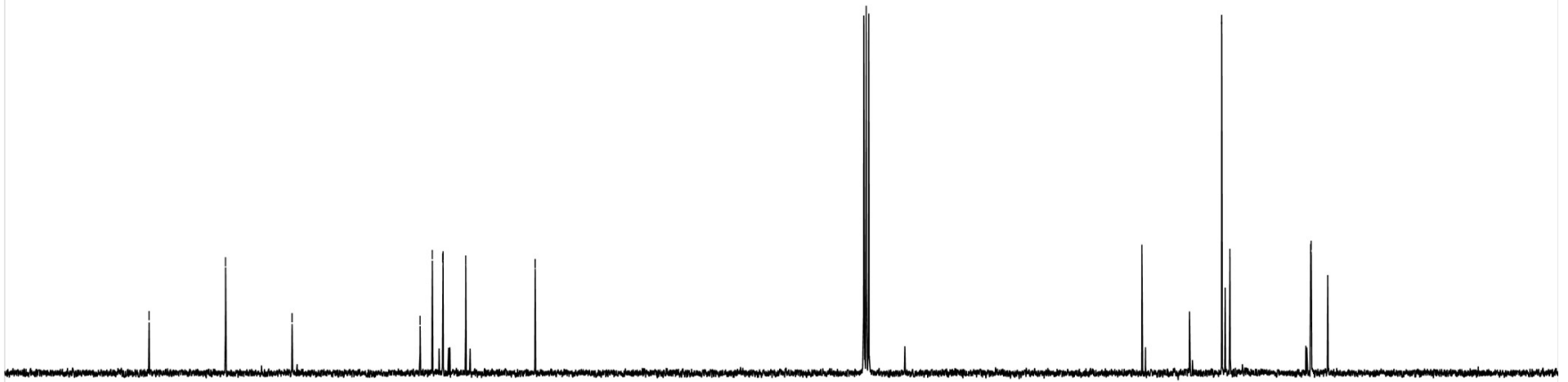




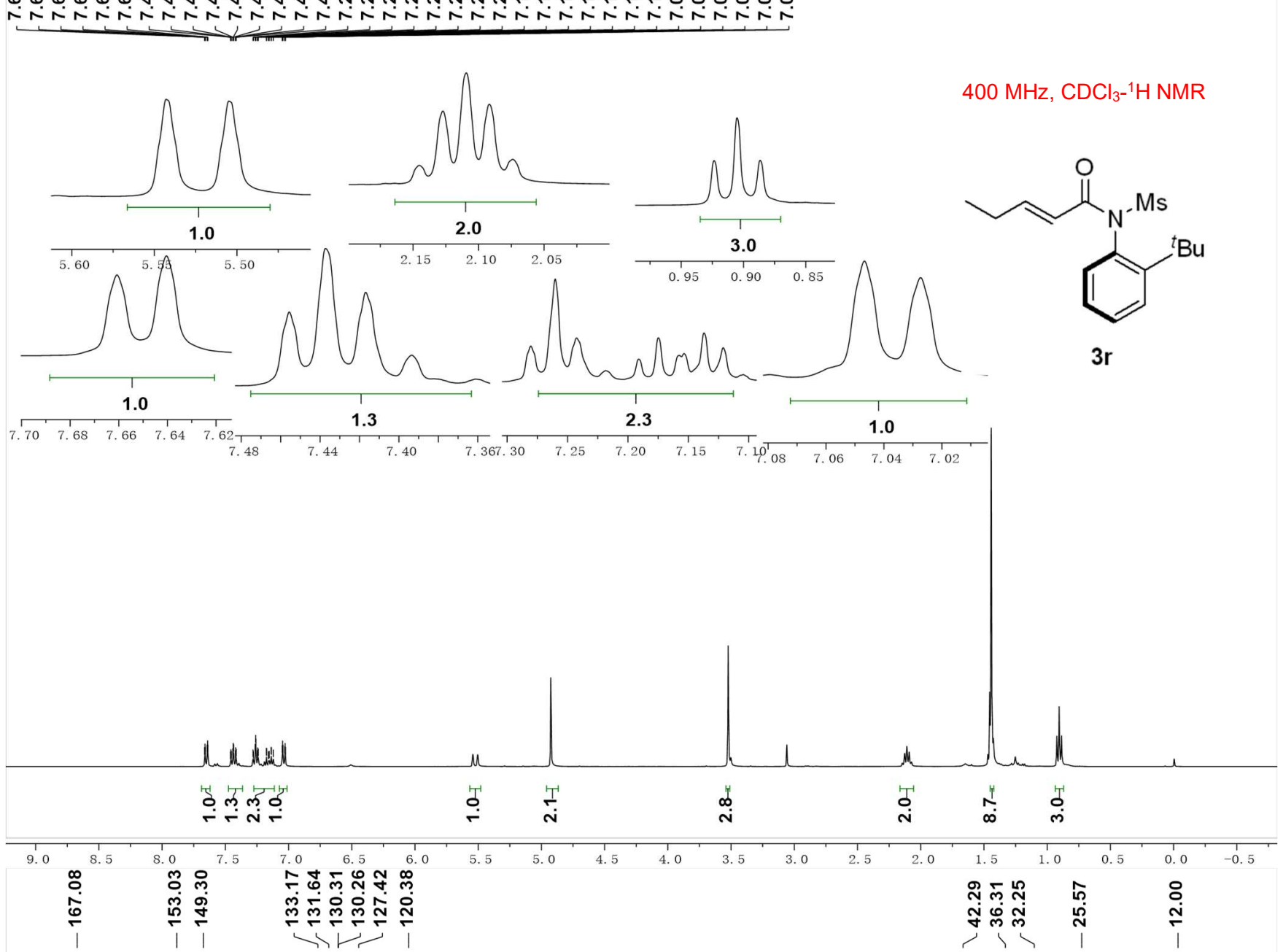

$101 \mathrm{MHz}, \mathrm{CDCl}_{3}-{ }^{13} \mathrm{C}$ NMR
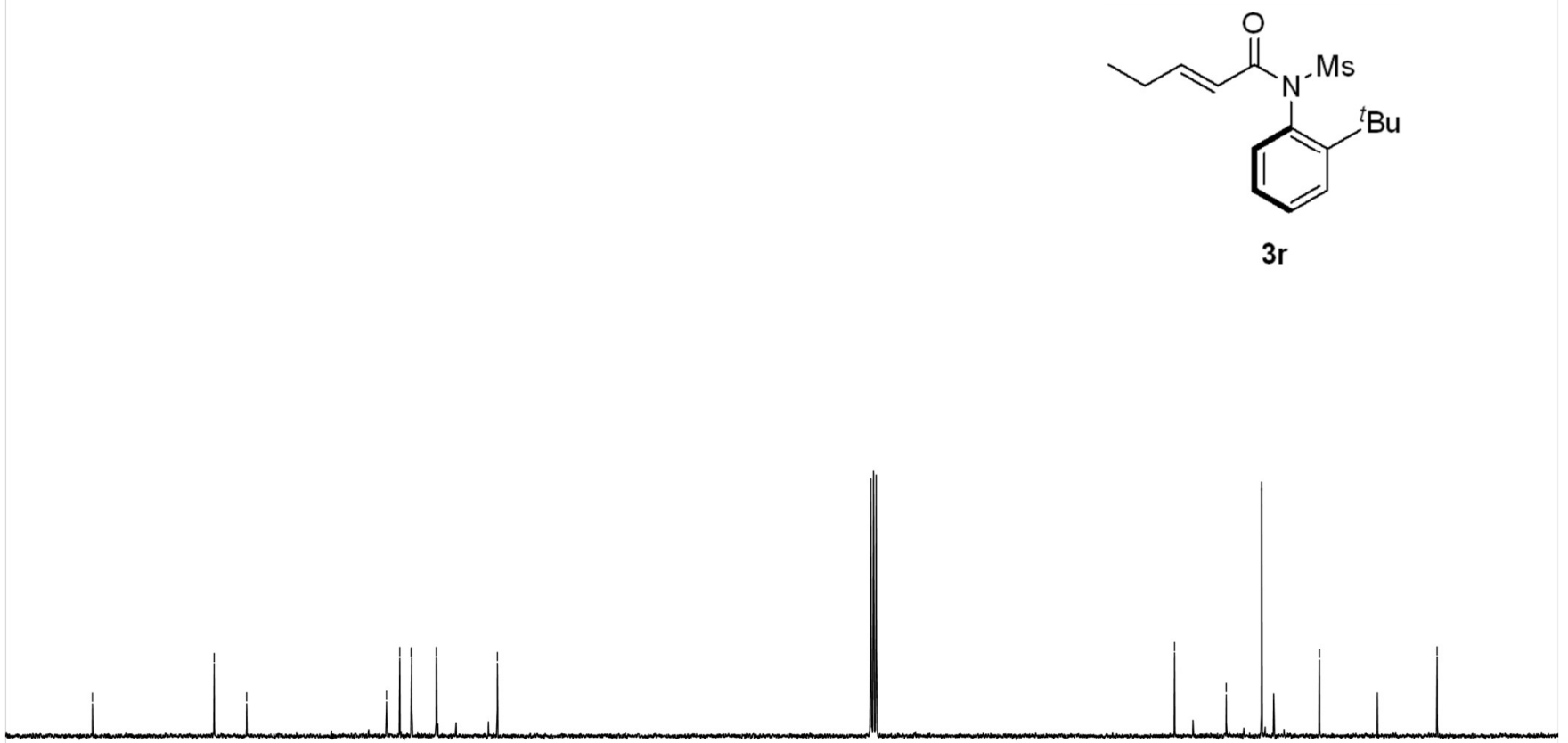


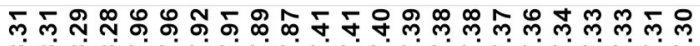

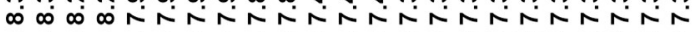

$\stackrel{\circ}{i}$

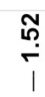

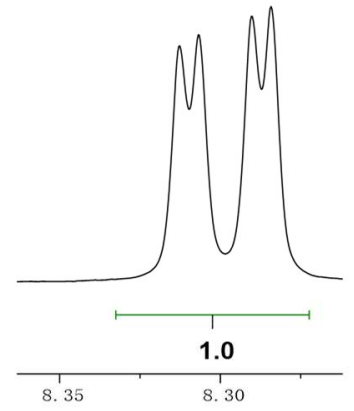

$400 \mathrm{MHz}, \mathrm{CDCl}_{3}-{ }^{-1} \mathrm{H} \mathrm{NMR}$

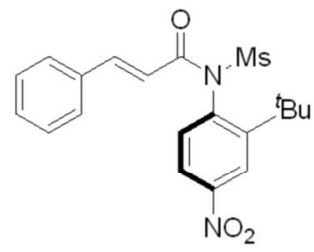

3s
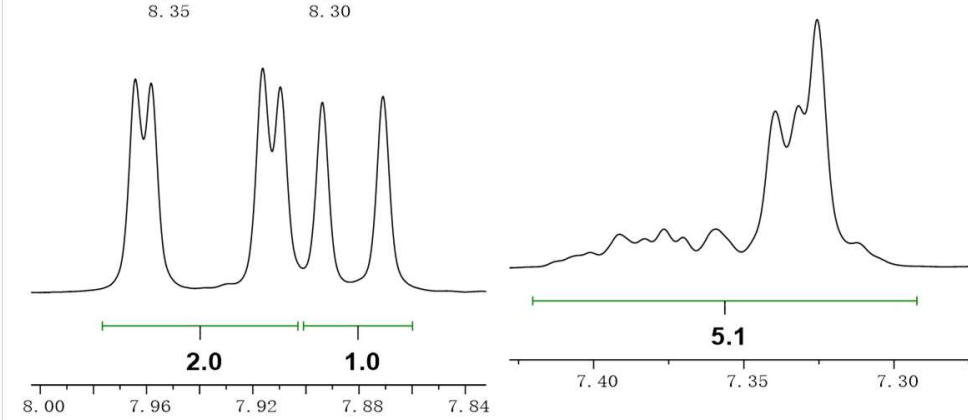

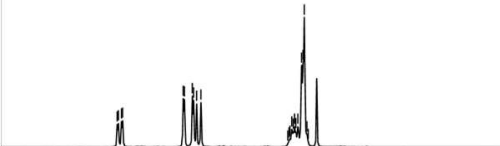

宁

$\mu$

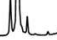

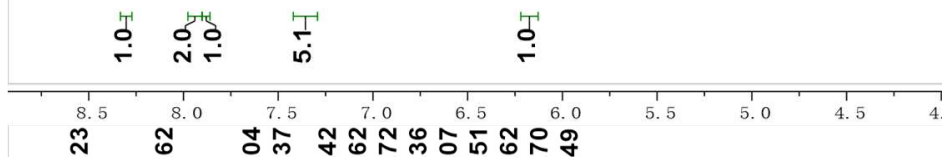

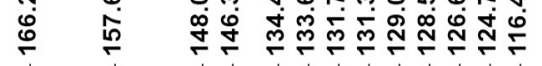

i

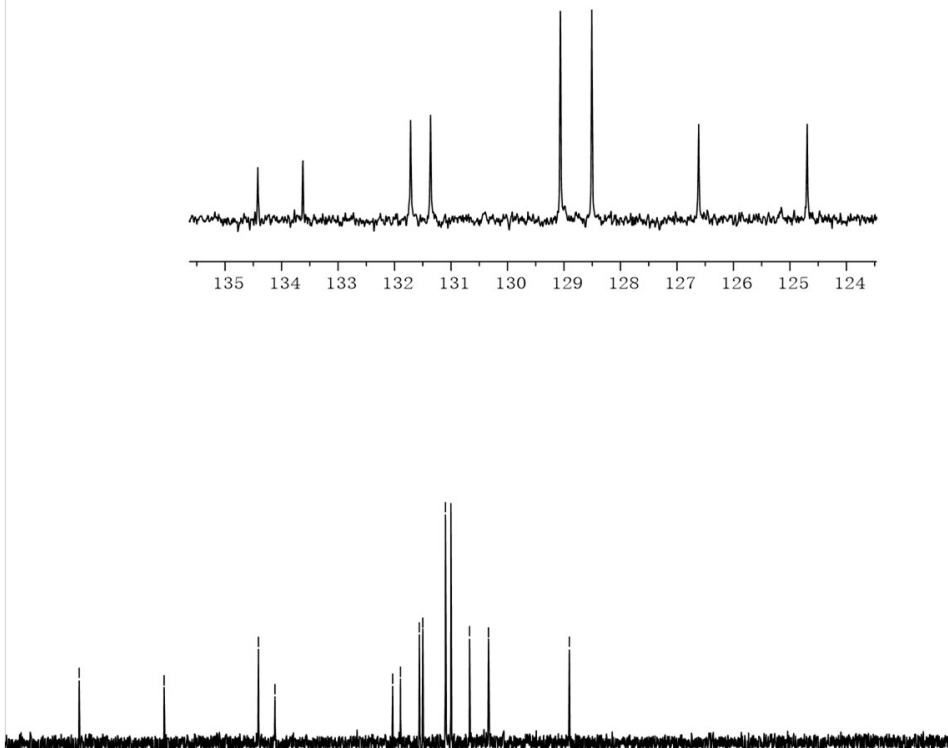

ì

范

$101 \mathrm{MHz}, \mathrm{CDCl}_{3}{ }^{-13} \mathrm{C}$ NMR

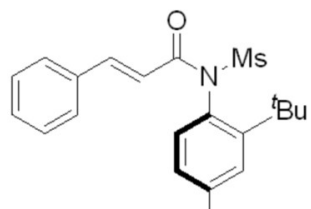

$\mathrm{NO}_{2}$

3s 


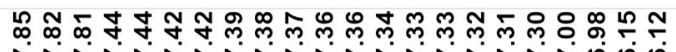

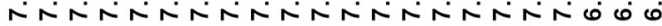
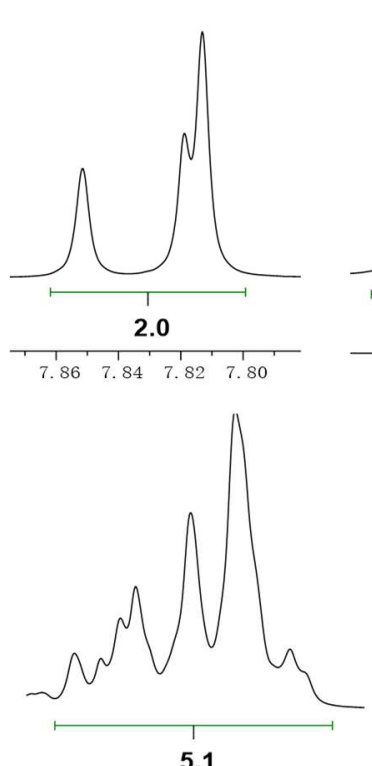

5.1
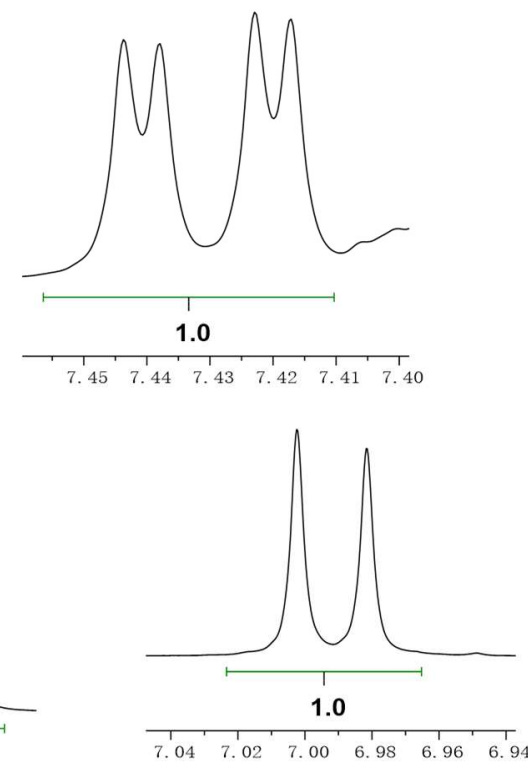

$\begin{array}{lllllll}7^{\prime} .40 & 7.38 & 7.36 & 7.34 & 7.32 & 7.30\end{array}$
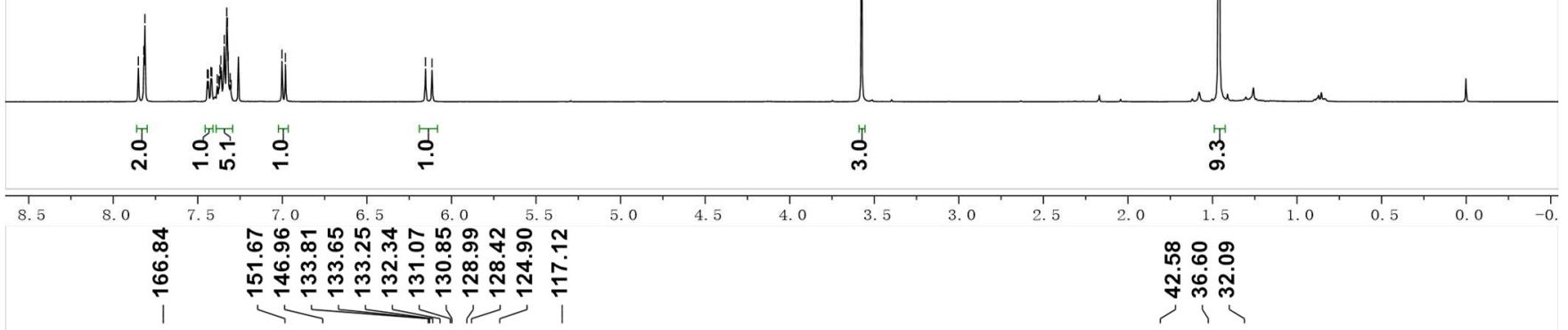

$101 \mathrm{MHz}, \mathrm{CDCl}_{3}-{ }^{13} \mathrm{C}$ NMR

$3 t$

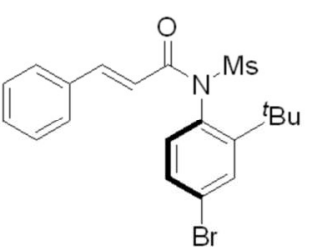

$3 t$
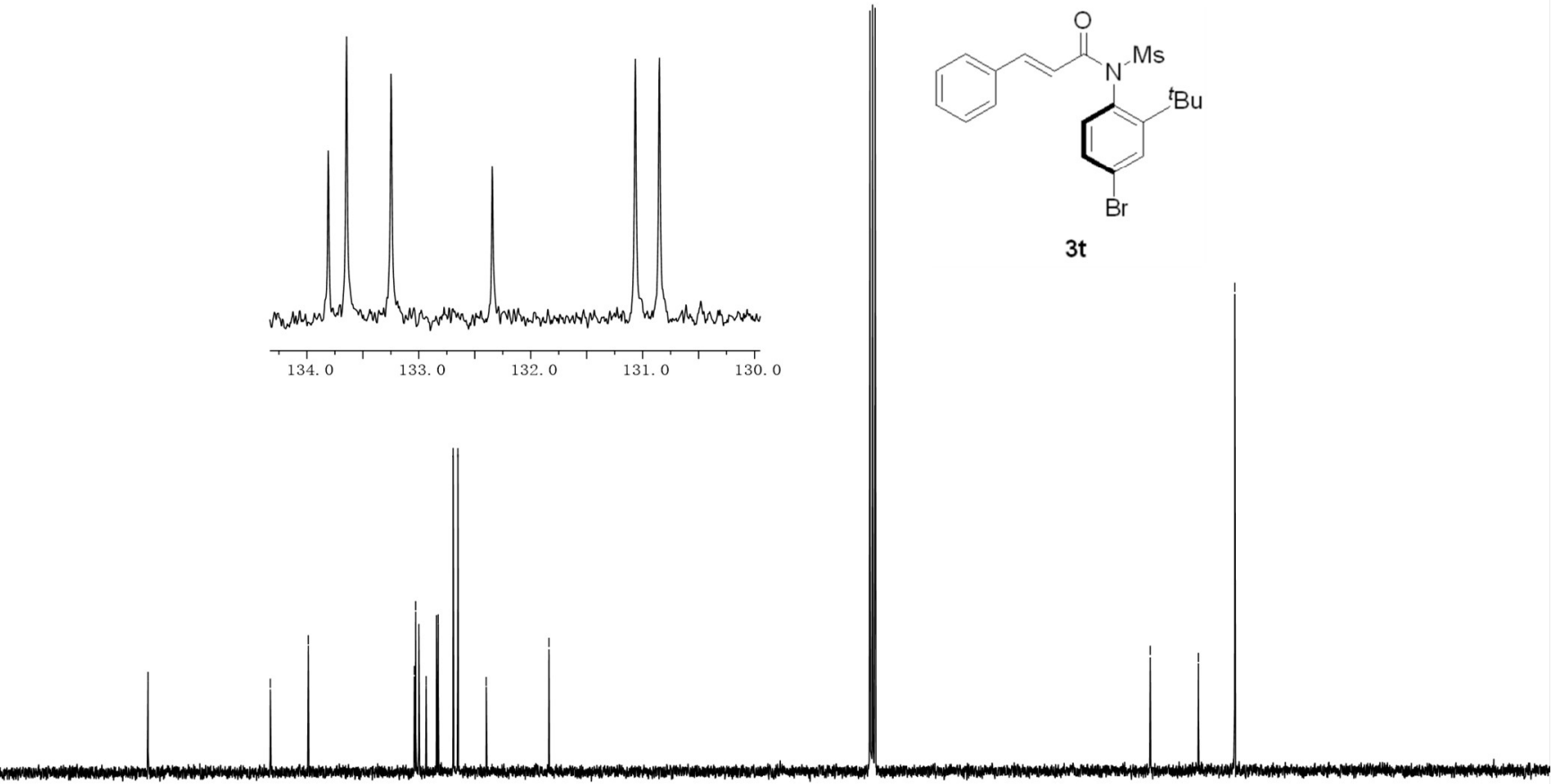


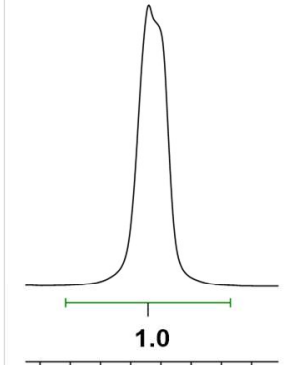

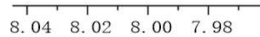

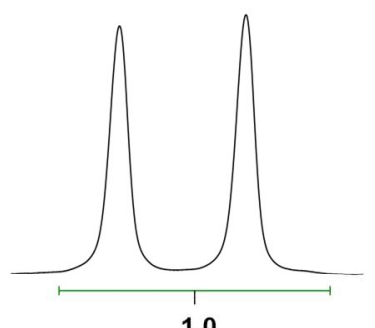

$\begin{array}{cccccc}7.88 & 7.86 & 7.84 & 7.82 & 7.80 & 7.78\end{array}$
$400 \mathrm{MHz}, \mathrm{CDCl}_{3}-{ }^{-1} \mathrm{H}$ NMR

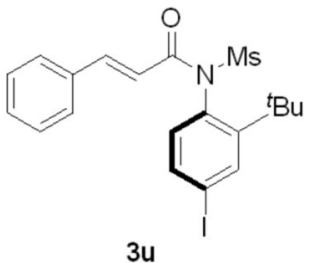

3u
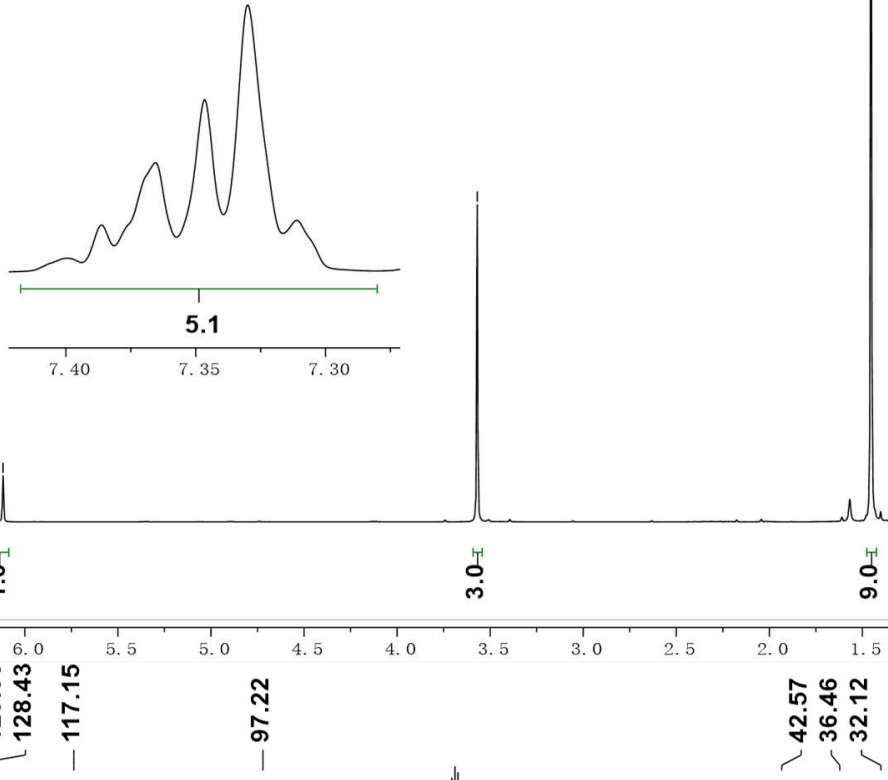

$101 \mathrm{MHz}, \mathrm{CDCl}_{3}{ }^{-13} \mathrm{C} \mathrm{NMR}$

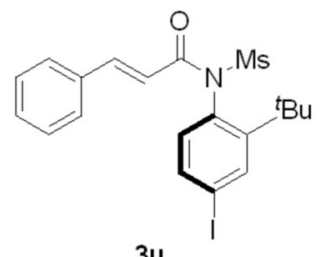

$3 u$ 

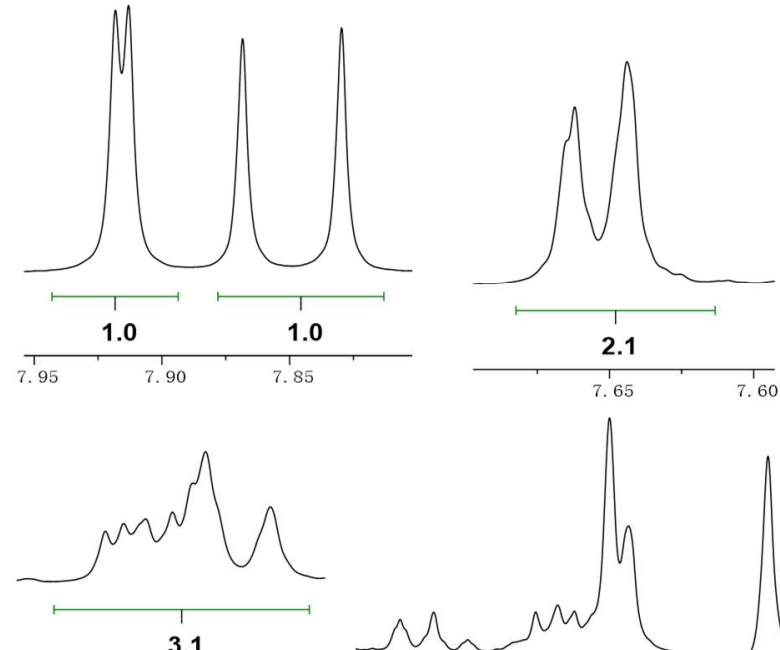

3.1
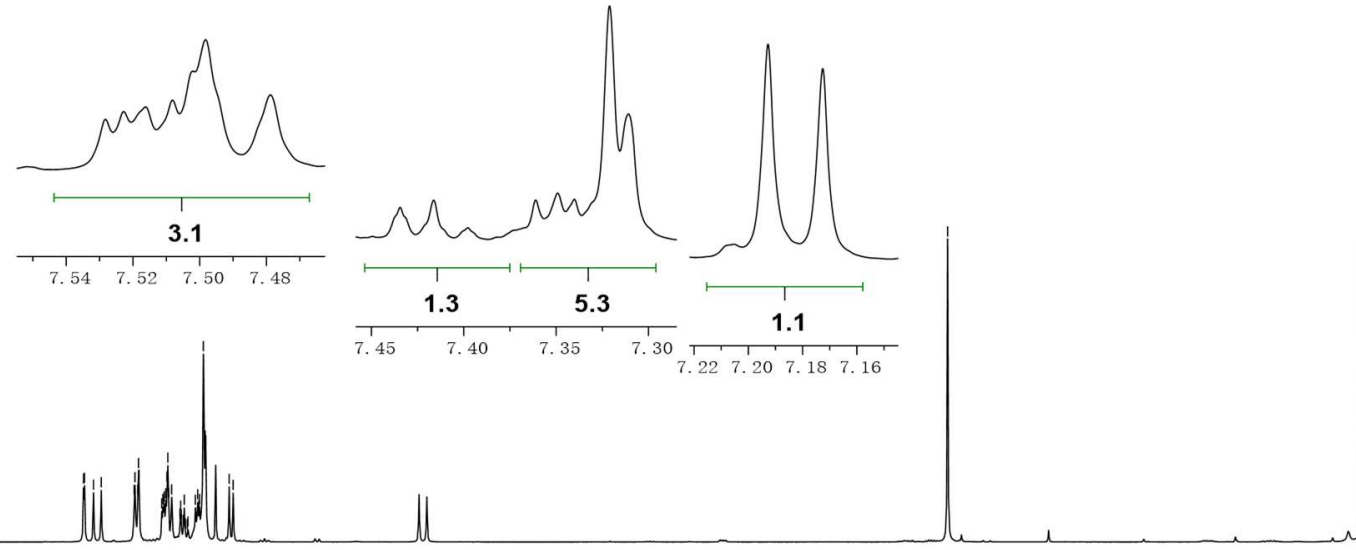

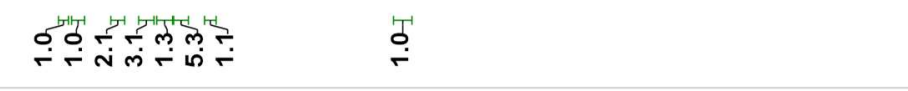

8.0

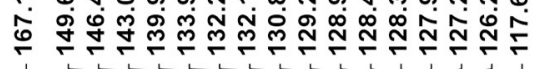

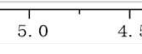

(n)

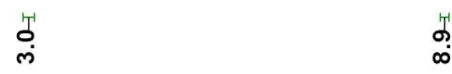

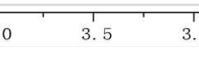

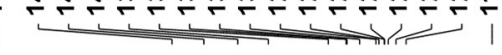

$101 \mathrm{MHz}, \mathrm{CDCl}_{3}{ }^{-13} \mathrm{C} \mathrm{NMR}$
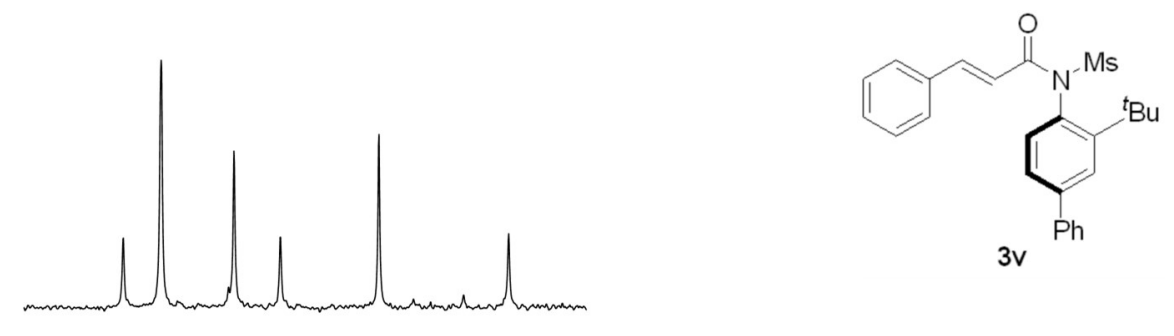

$\begin{array}{llllllllllllll}130.0 & 129.5 & 129.0 & 128.5 & 128.0 & 127.5 & 127.0 & 126.5 & 126.0\end{array}$

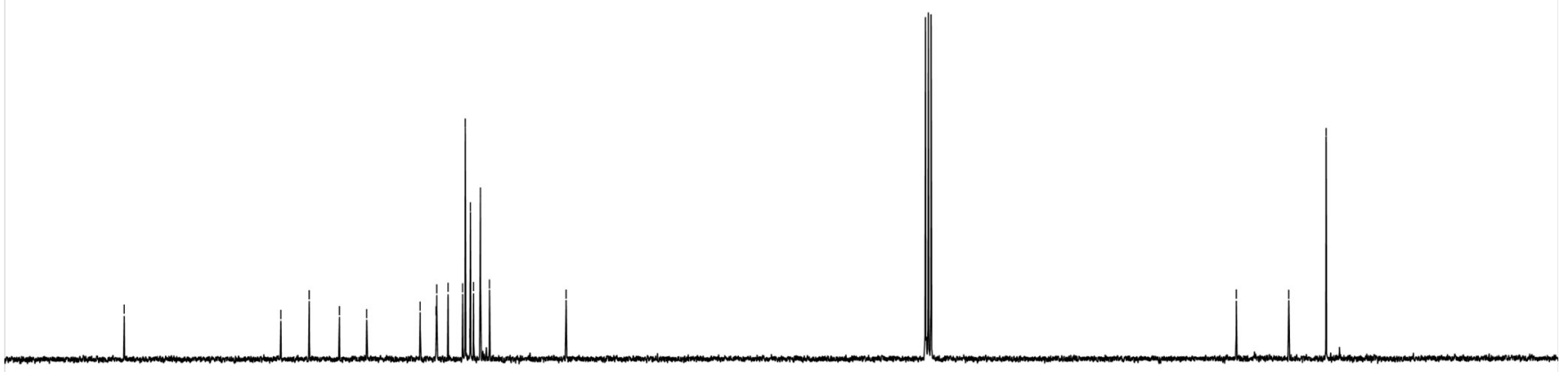




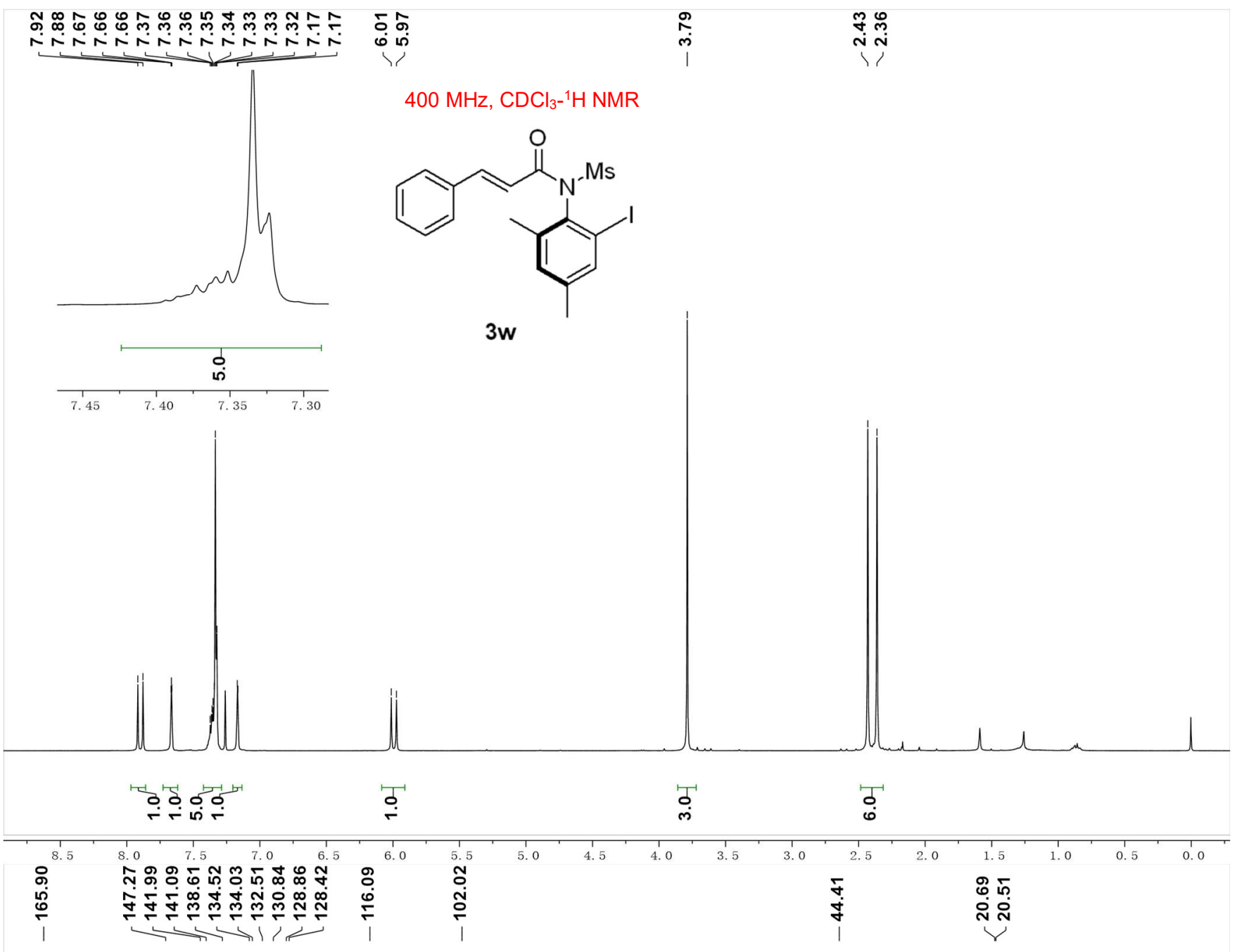

$101 \mathrm{MHz}, \mathrm{CDCl}_{3^{-}}{ }^{13} \mathrm{C}$ NMR<smiles>Cc1cc(C)c(N(C(=O)/C=C/c2ccccc2)S(C)(=O)=O)c(I)c1</smiles>

$3 w$
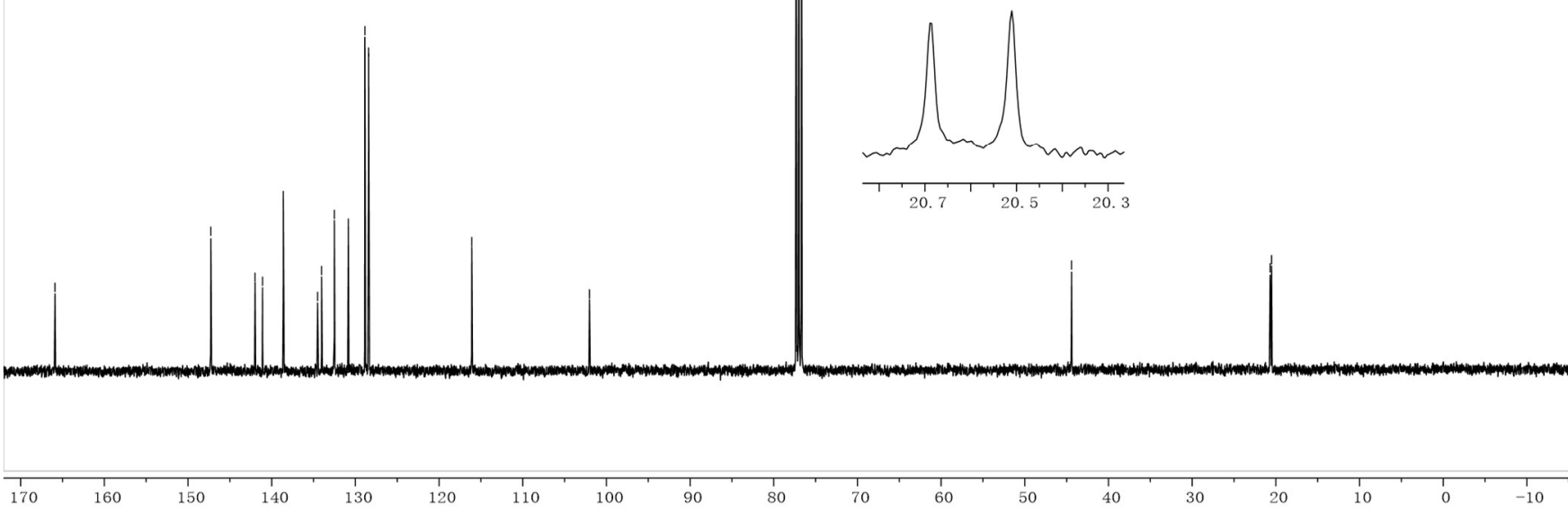

96 


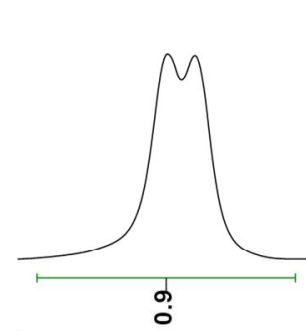

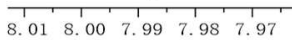
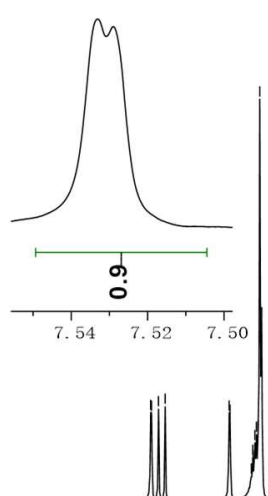

कूँ
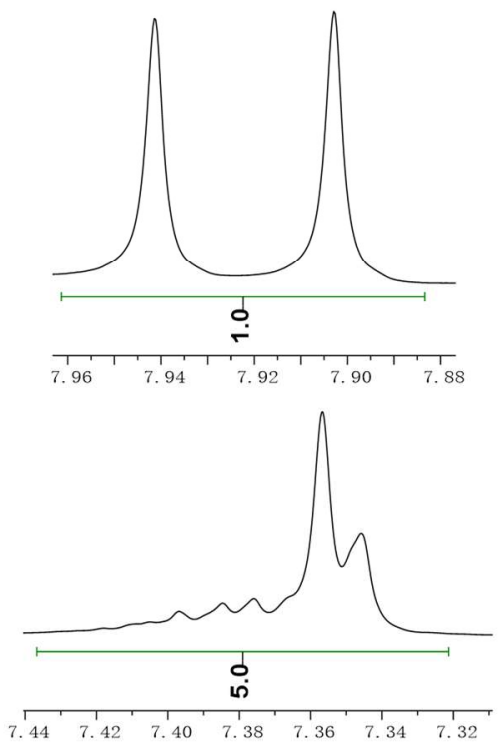

$400 \mathrm{MHz}, \mathrm{CDCl}_{3}{ }^{-1} \mathrm{H} \mathrm{NMR}$<smiles>Cc1cc(Br)cc(I)c1N(C(=O)/C=C/c1ccccc1)S(C)(=O)=O</smiles>

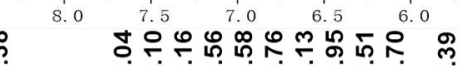

il

옹

$101 \mathrm{MHz}, \mathrm{CDCl}_{3}-{ }^{13} \mathrm{C}$ NMR
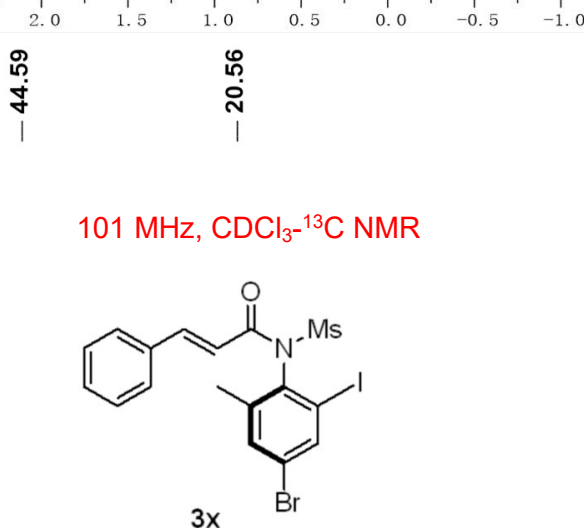

(c)

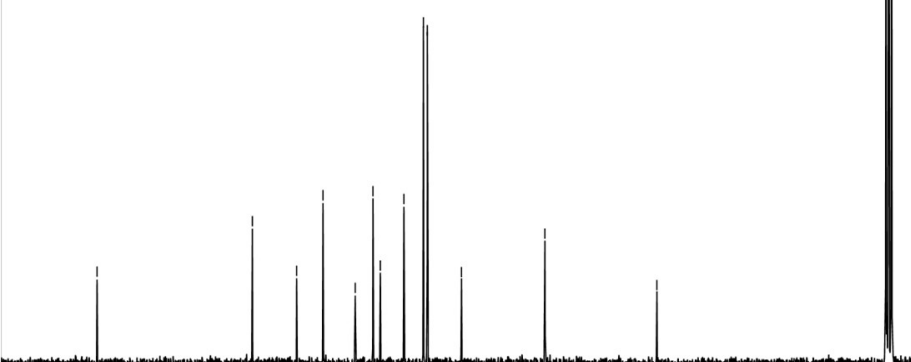

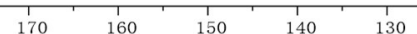
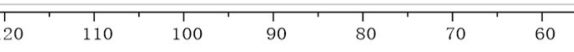

50

20 
$400 \mathrm{MHz}, \mathrm{CDCl}_{3}-{ }^{-1} \mathrm{H} \mathrm{NMR}$

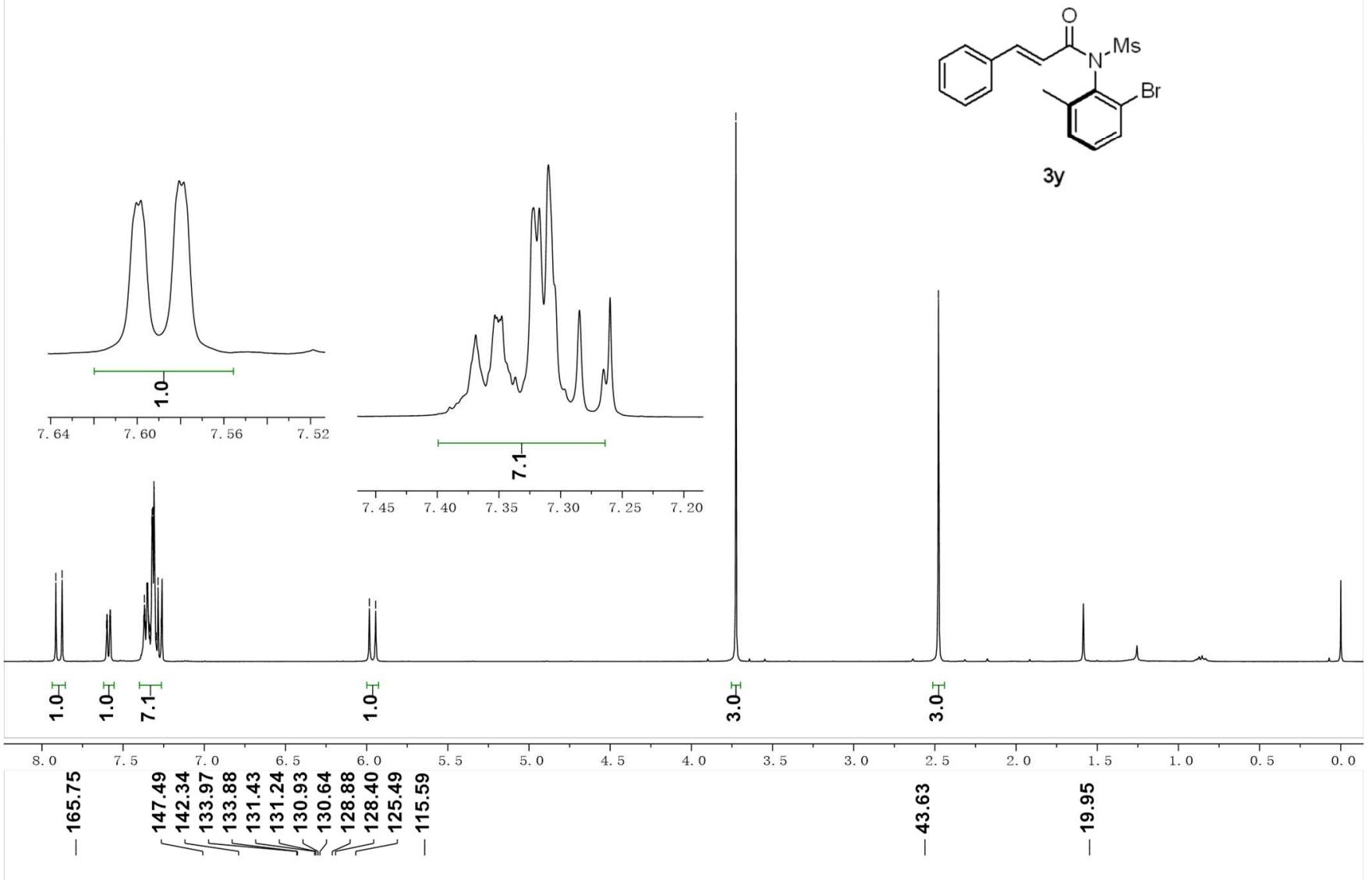

$101 \mathrm{MHz}, \mathrm{CDCl}_{3}{ }^{-13} \mathrm{C} \mathrm{NMR}$

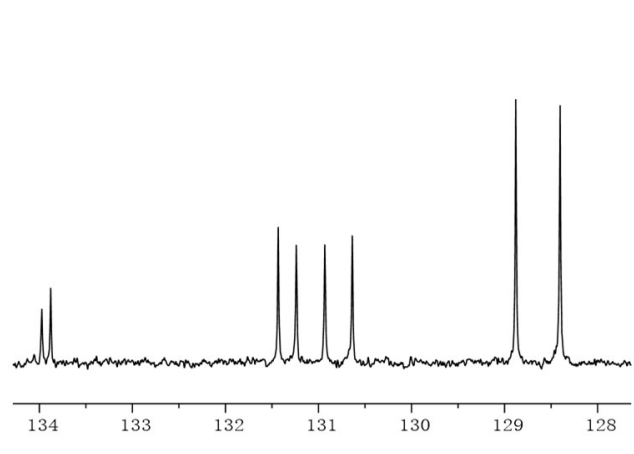<smiles>CC(=O)N(C(=O)/C=C/c1ccccc1)c1c(C)cccc1Br</smiles>

$3 y$
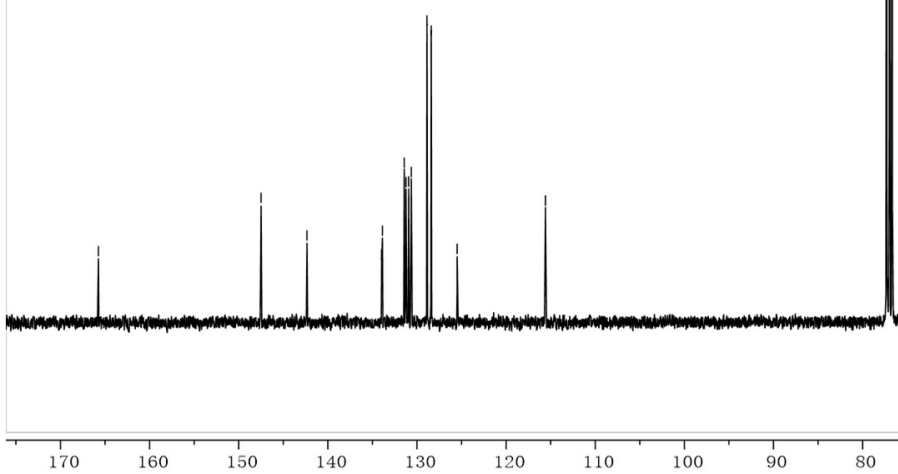

70

60 


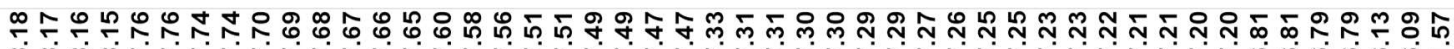

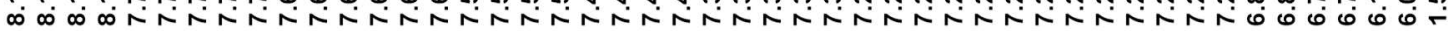
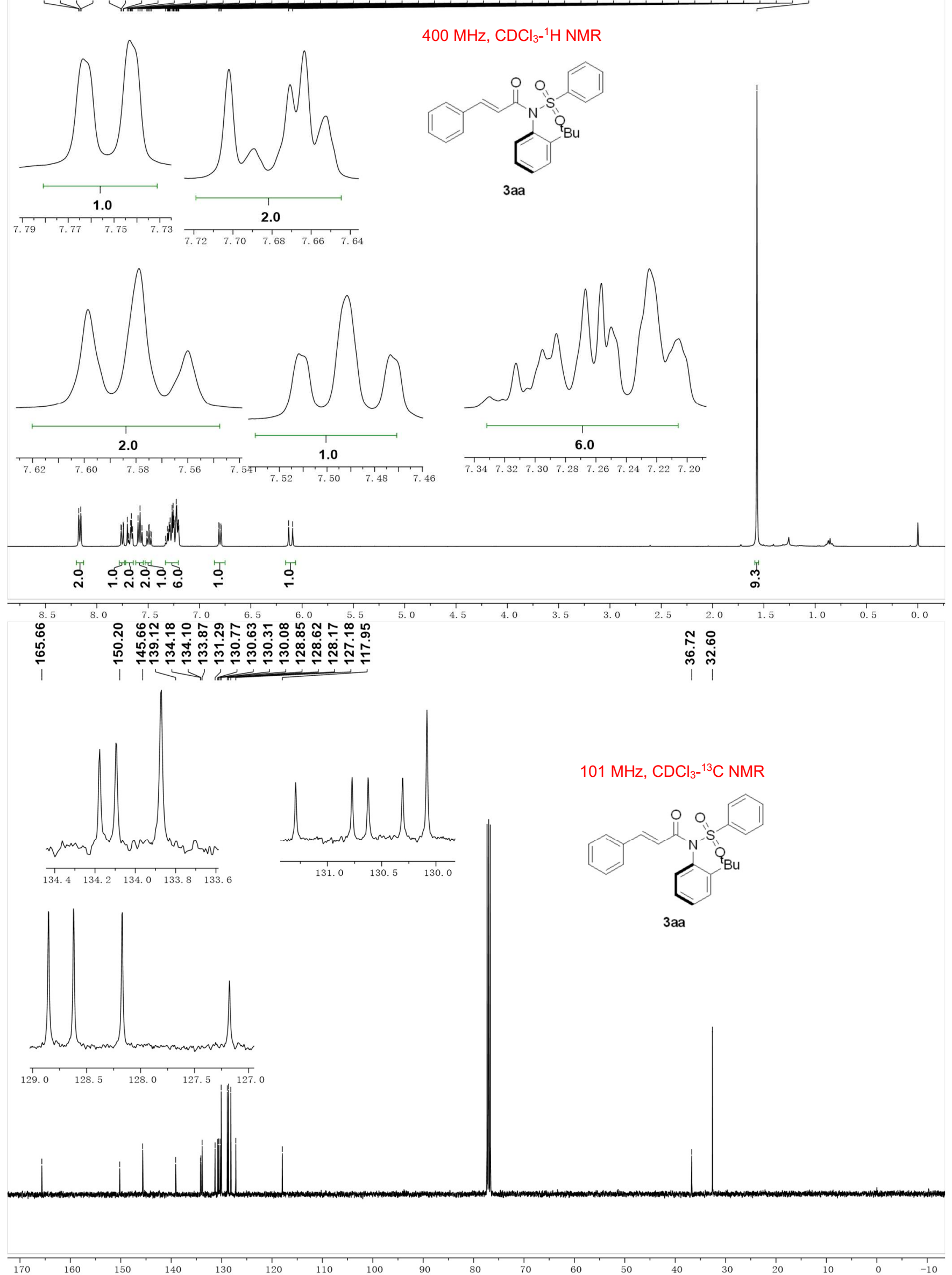

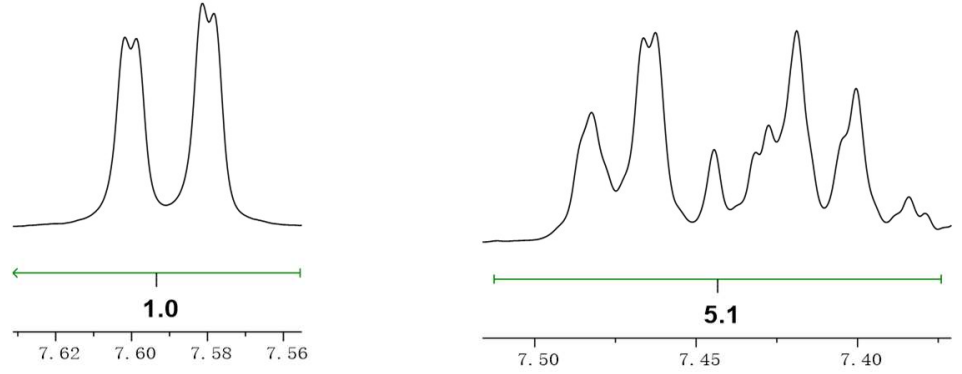

$400 \mathrm{MHz}, \mathrm{CDCl}_{3}{ }^{-1} \mathrm{H}$ NMR
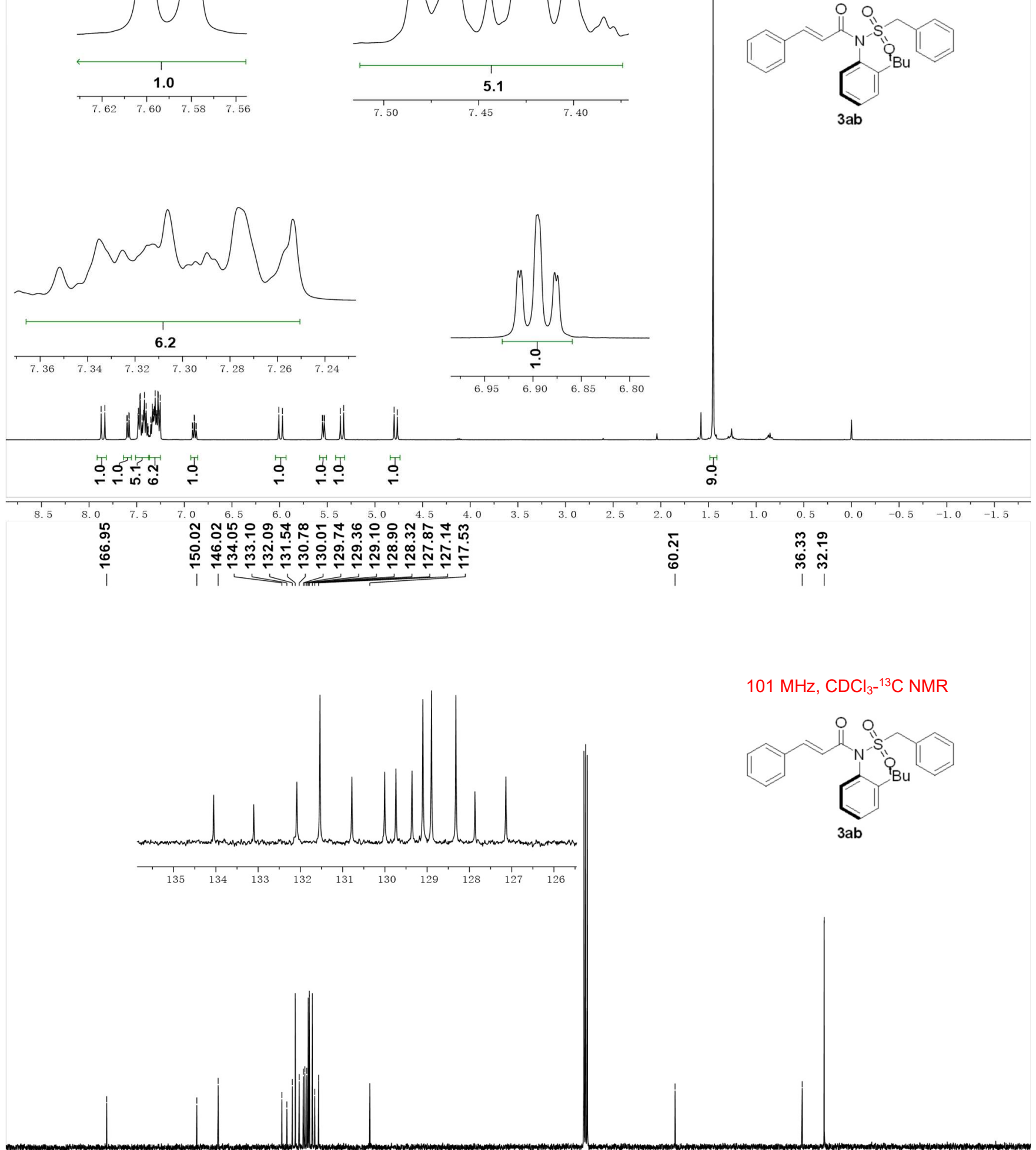

$101 \mathrm{MHz}, \mathrm{CDCl}_{3}{ }^{-13} \mathrm{C} \mathrm{NMR}$ 
$400 \mathrm{MHz}, \mathrm{CDCl}_{3}{ }^{-1} \mathrm{H} \mathrm{NMR}$

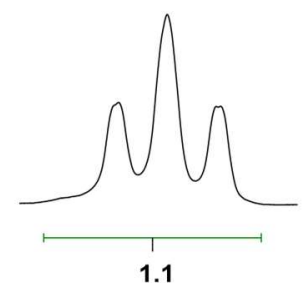

$\frac{}{1.1}$
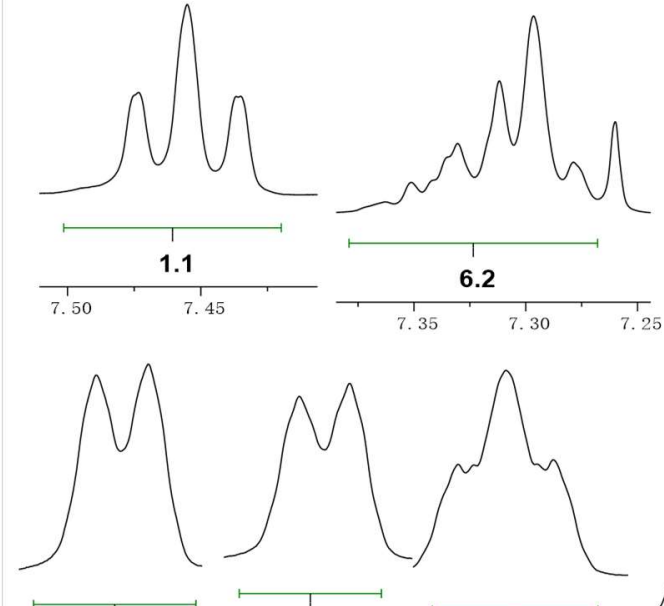

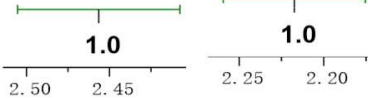
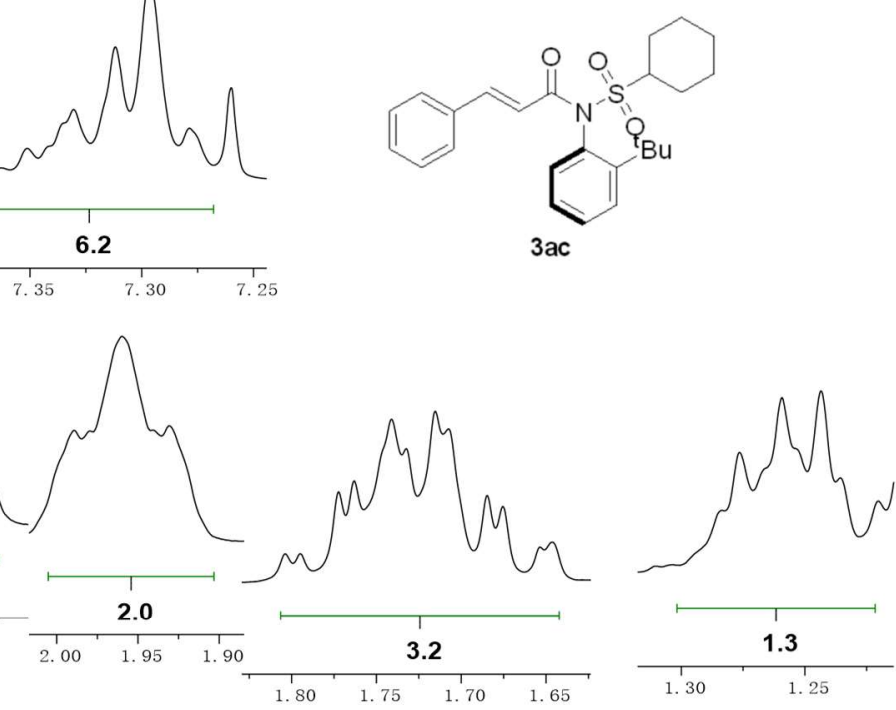

undu

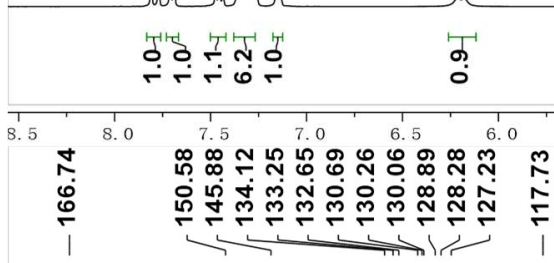

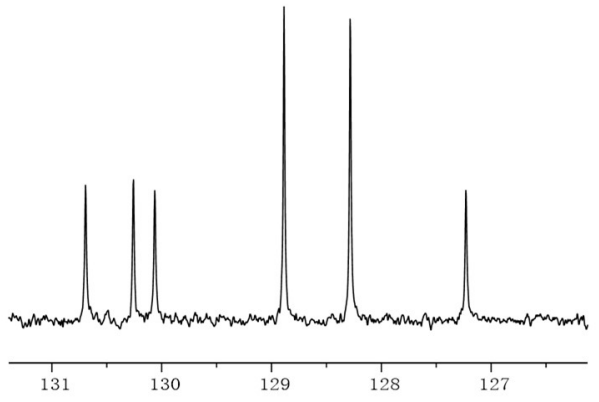
虬

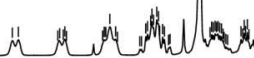
$\stackrel{?}{\circ}$

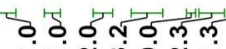
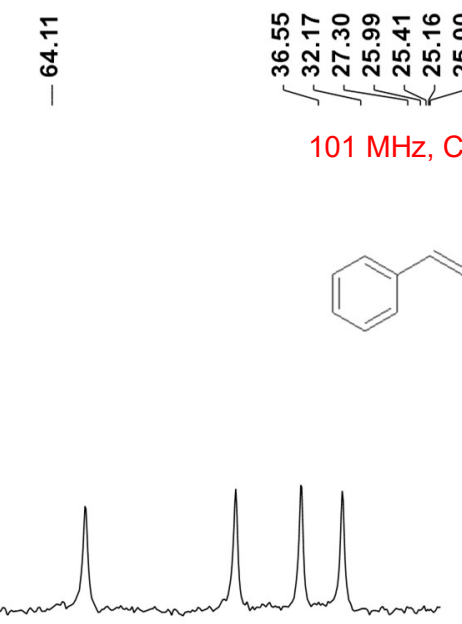

26. 226.025 .825 .625 .425 .225 .024 .8
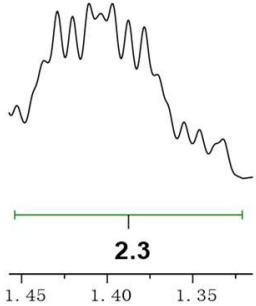

$101 \mathrm{MHz}, \mathrm{CDCl}_{3} \mathbf{-}^{13} \mathrm{C} \mathrm{NMR}$
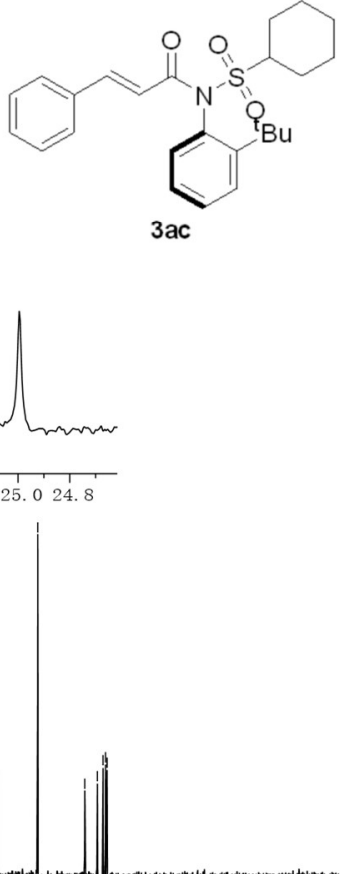

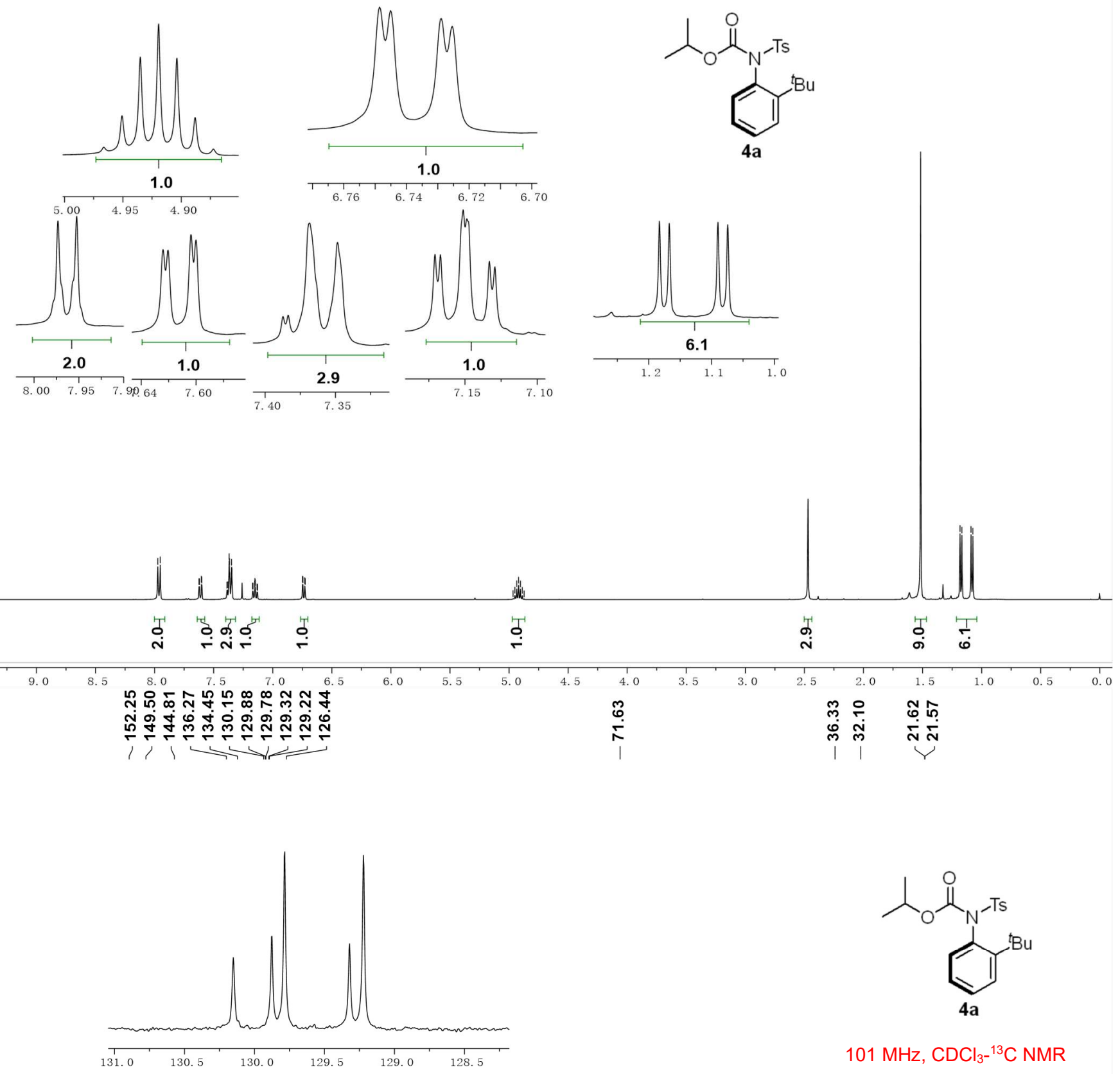

$101 \mathrm{MHz}, \mathrm{CDCl}_{3}-{ }^{13} \mathrm{C} \mathrm{NMR}$

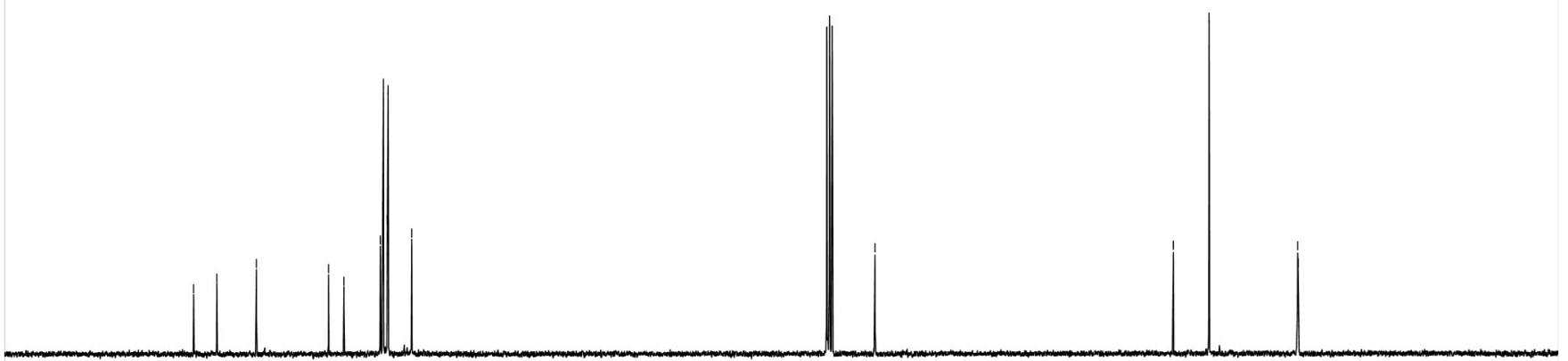



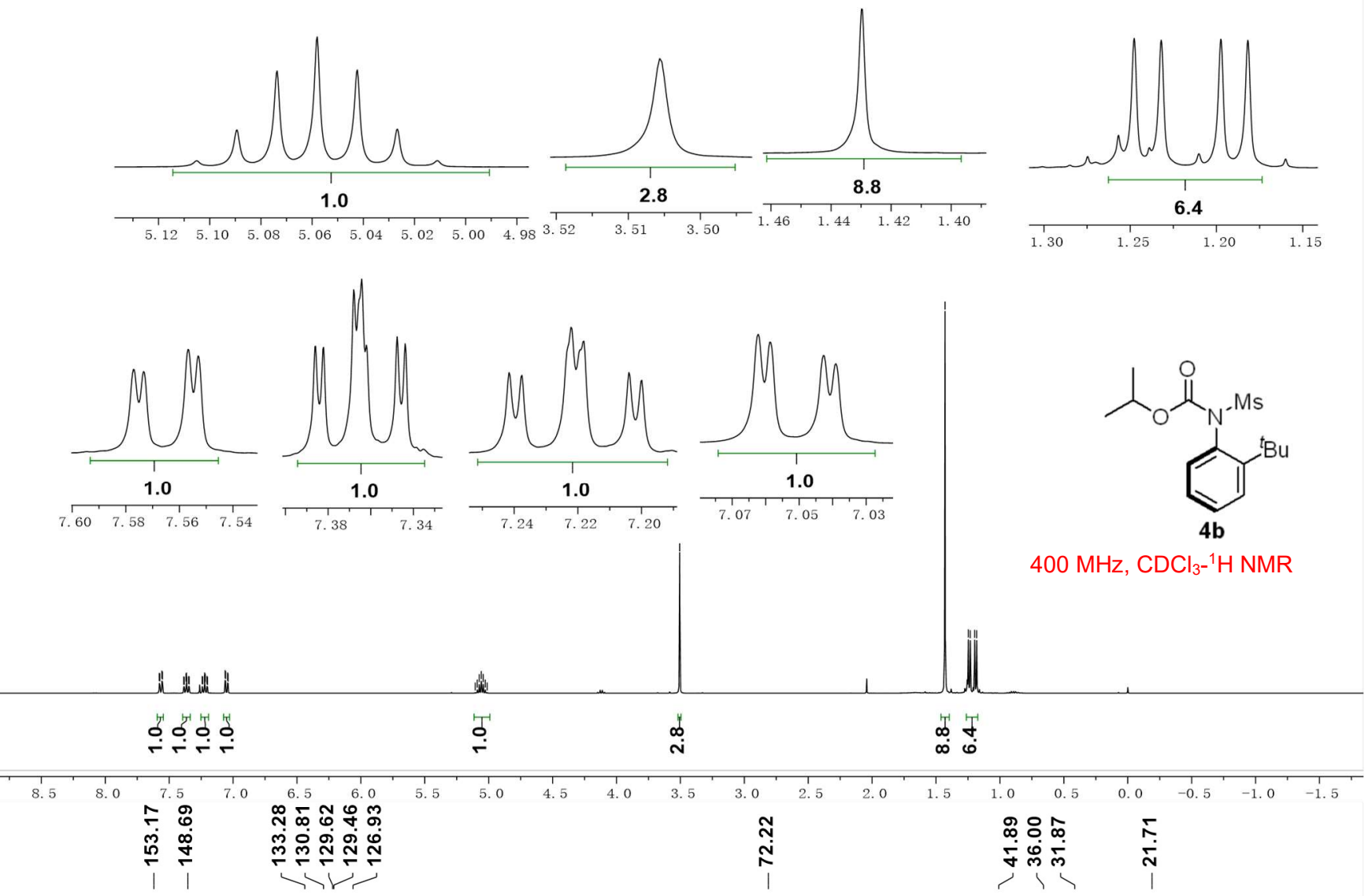

$101 \mathrm{MHz}, \mathrm{CDCl}_{3}-{ }^{13} \mathrm{C}$ NMR
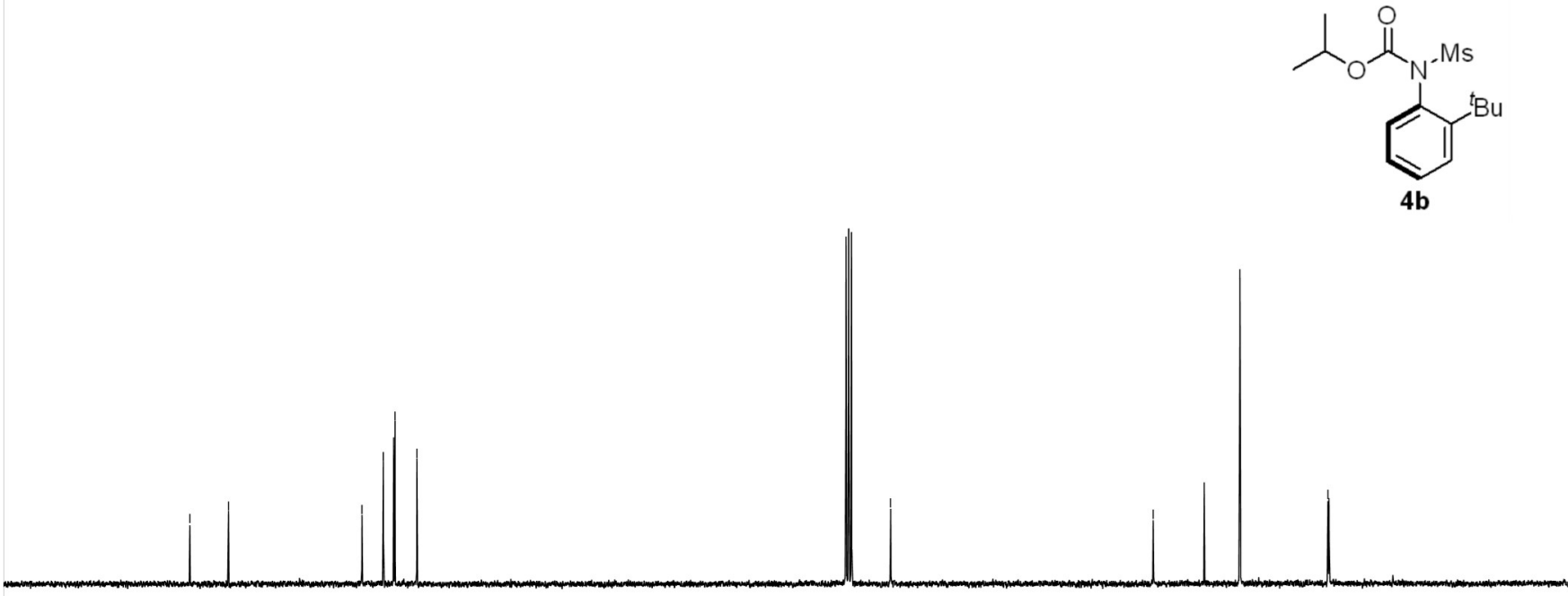

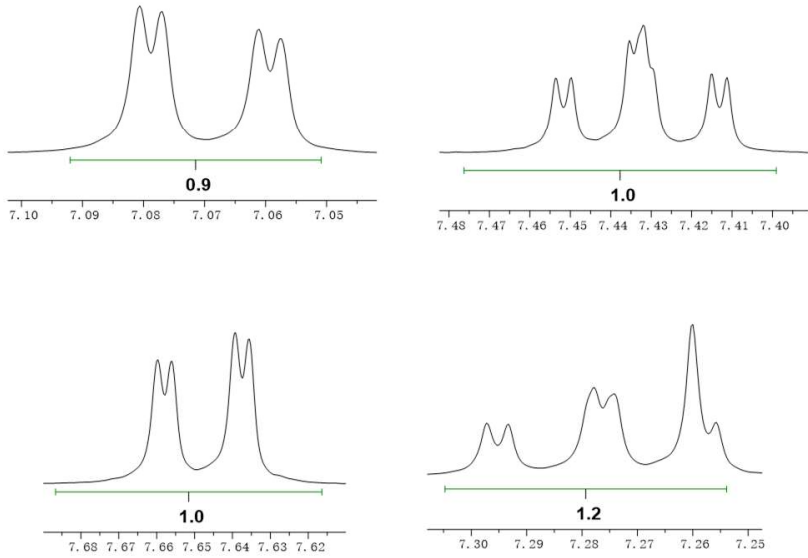

$400 \mathrm{MHz}, \mathrm{CDCl}_{3}-^{-1} \mathrm{H} \mathrm{NMR}$<smiles>CC(=O)N(c1ccc(C)cc1C(C)(C)C)S(C)(=O)=O</smiles>

$101 \mathrm{MHz}, \mathrm{CDCl}_{3}{ }^{-13} \mathrm{C} \mathrm{NMR}$<smiles>CC(=O)N(c1ccccc1)c1ccc(C)cc1Br</smiles>

กั

รูํำ

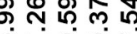

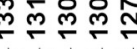
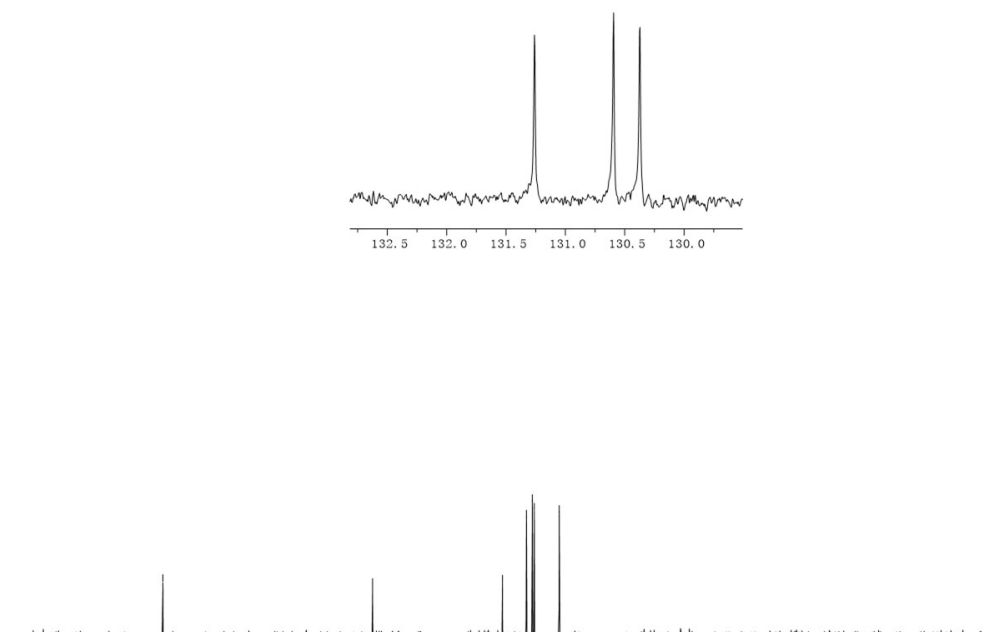

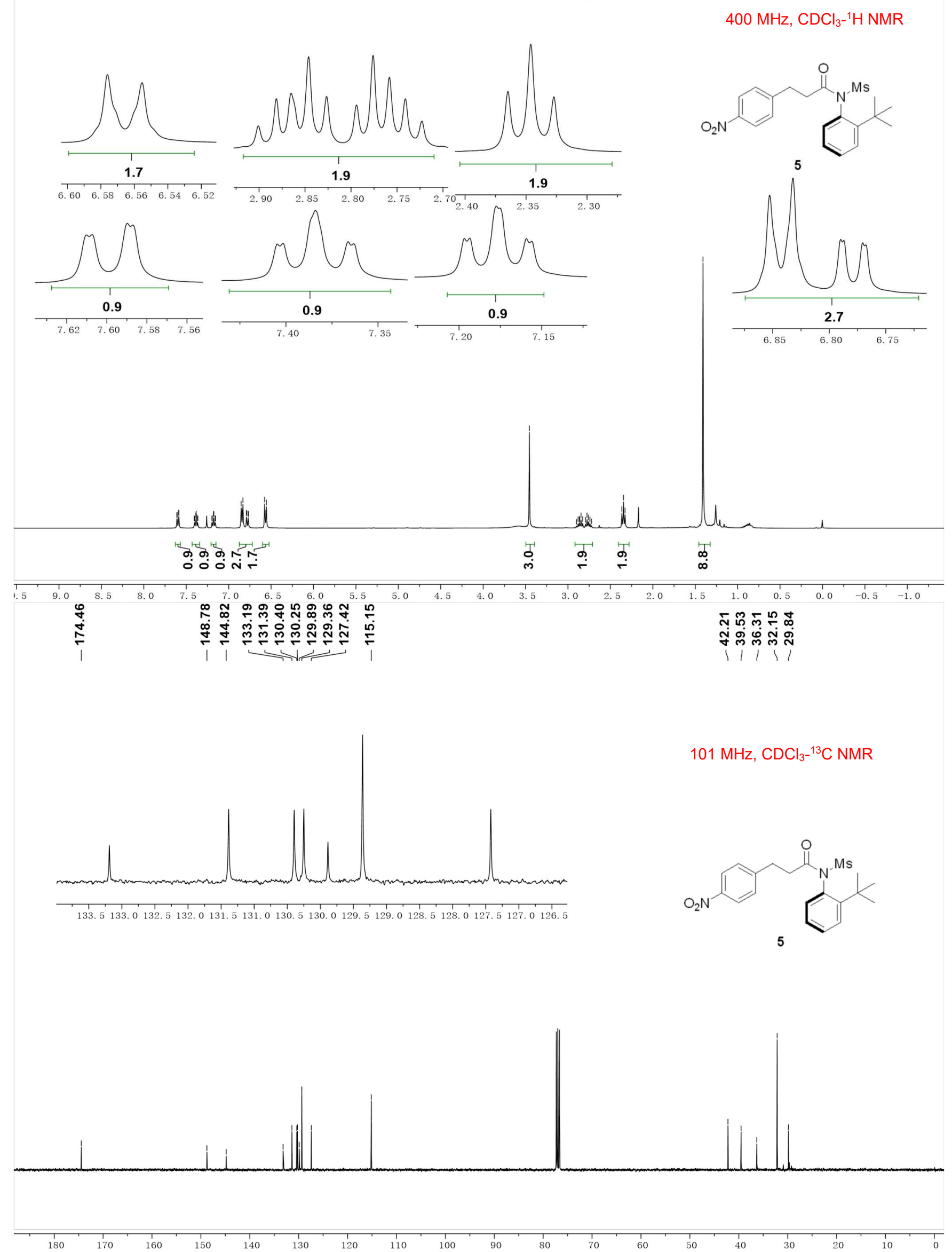
14. Copies of CD spectra in $\mathrm{MeOH}$
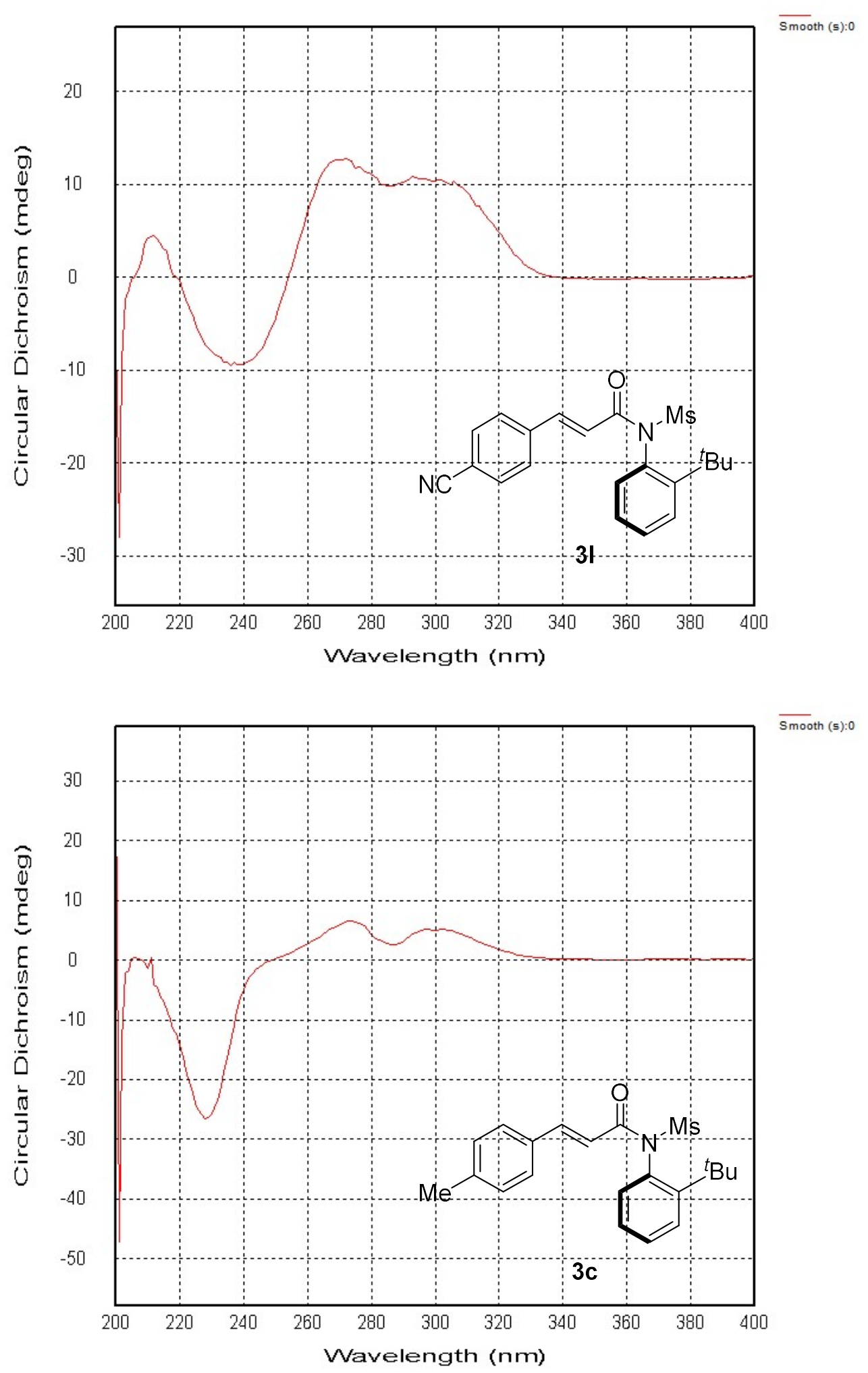


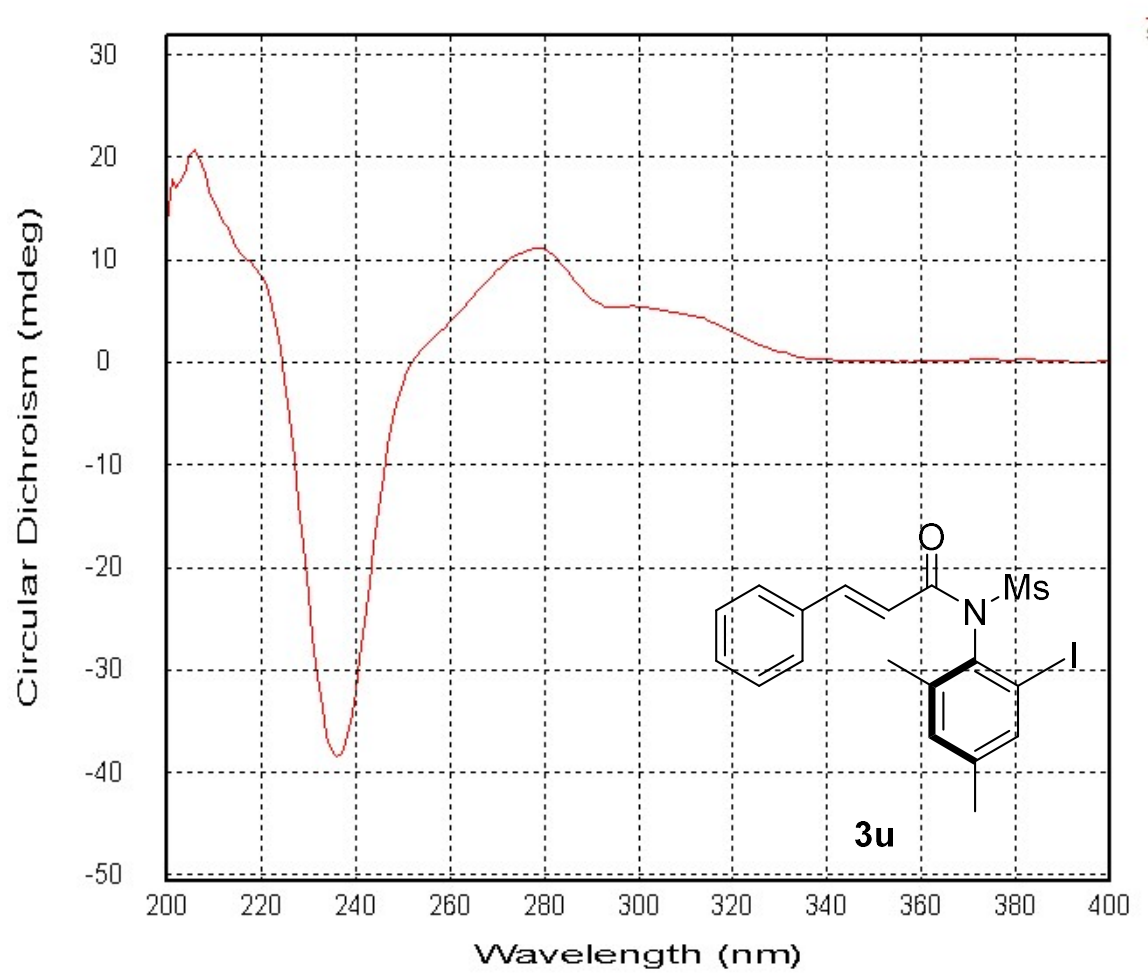

$\overline{\text { Smooth (s):0 }}$

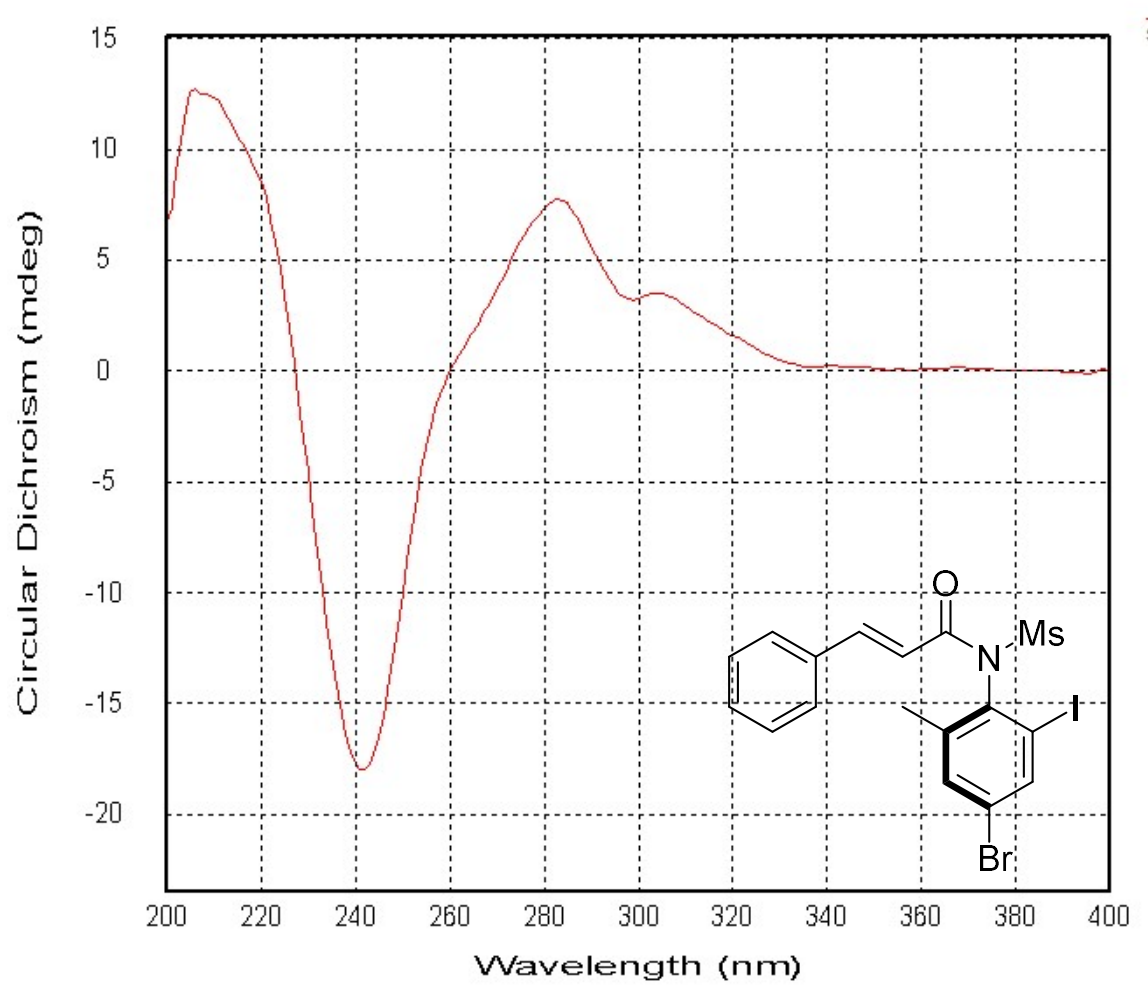

Smooth (s):0 


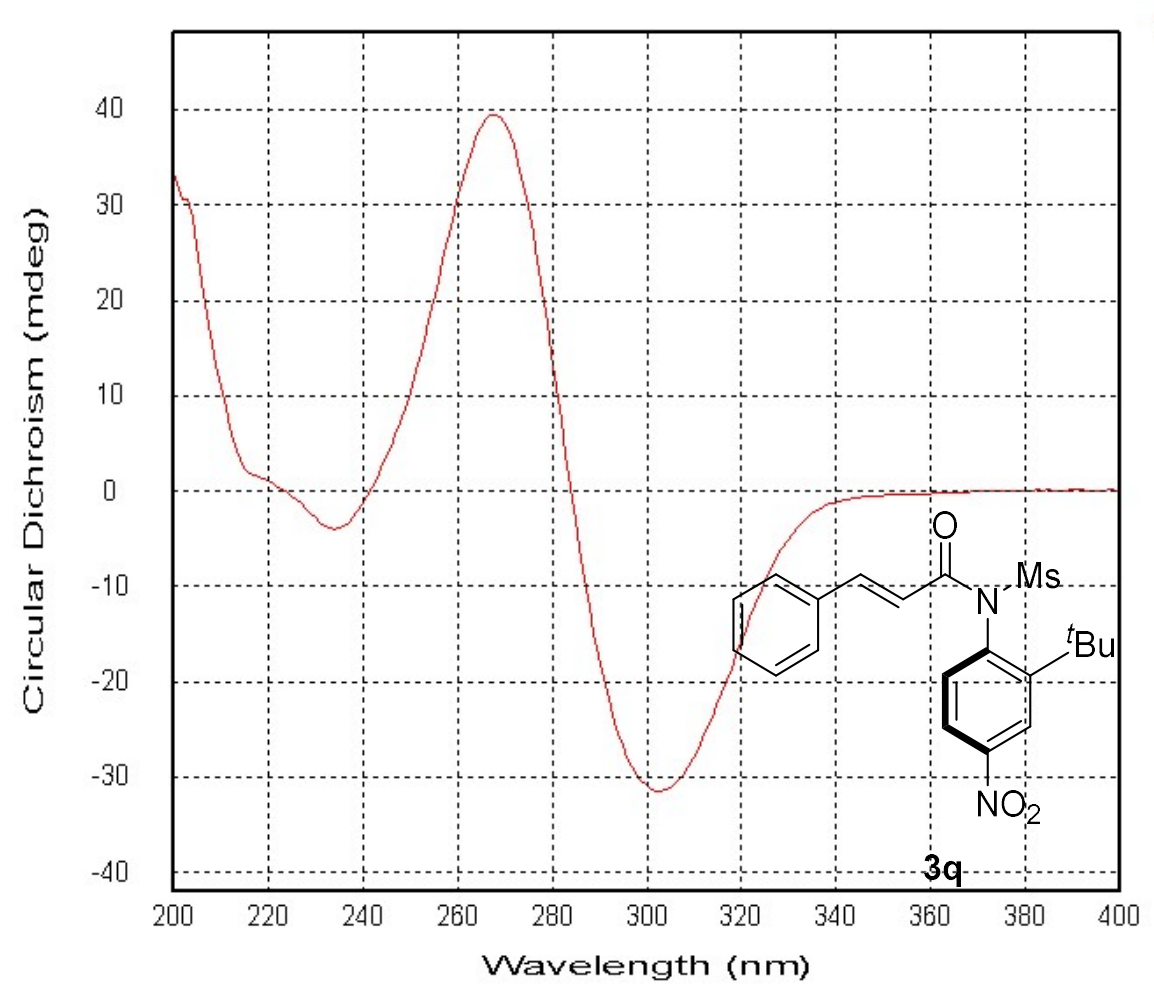

$\overline{\text { Smooth (s):0 }}$

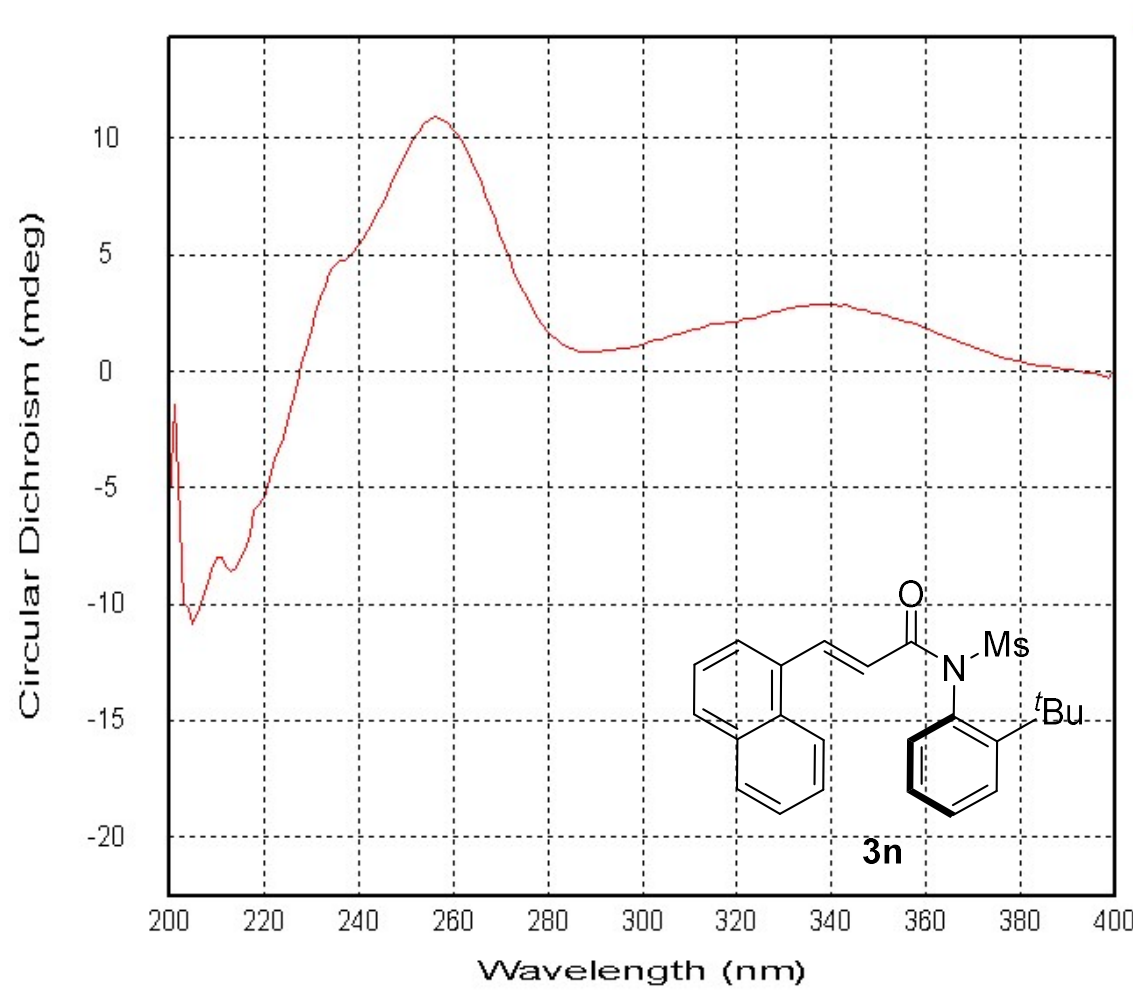

$\overline{\text { Smooth (s):0 }}$ 


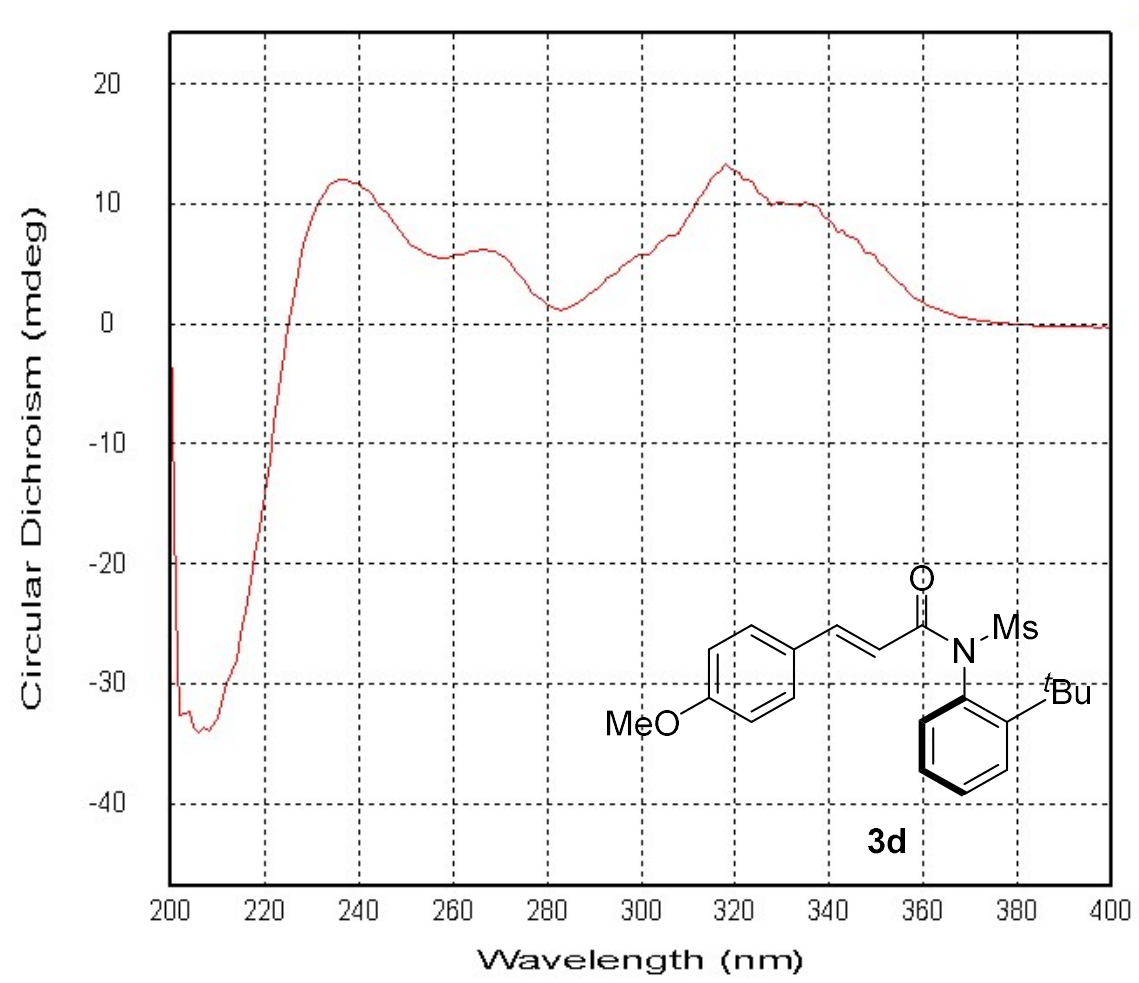

Smooth (s):0

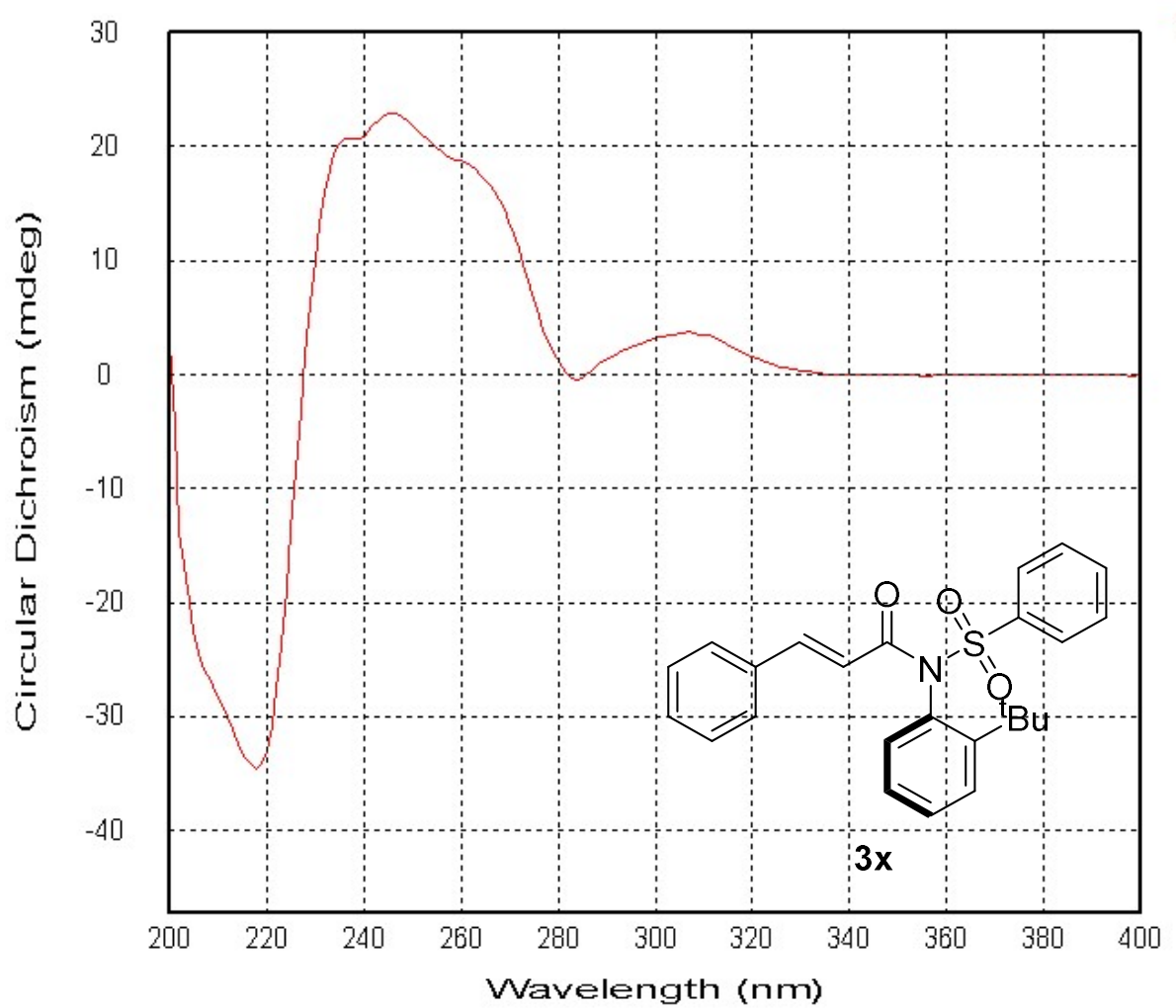

$\overline{\text { Smooth (s):0 }}$ 


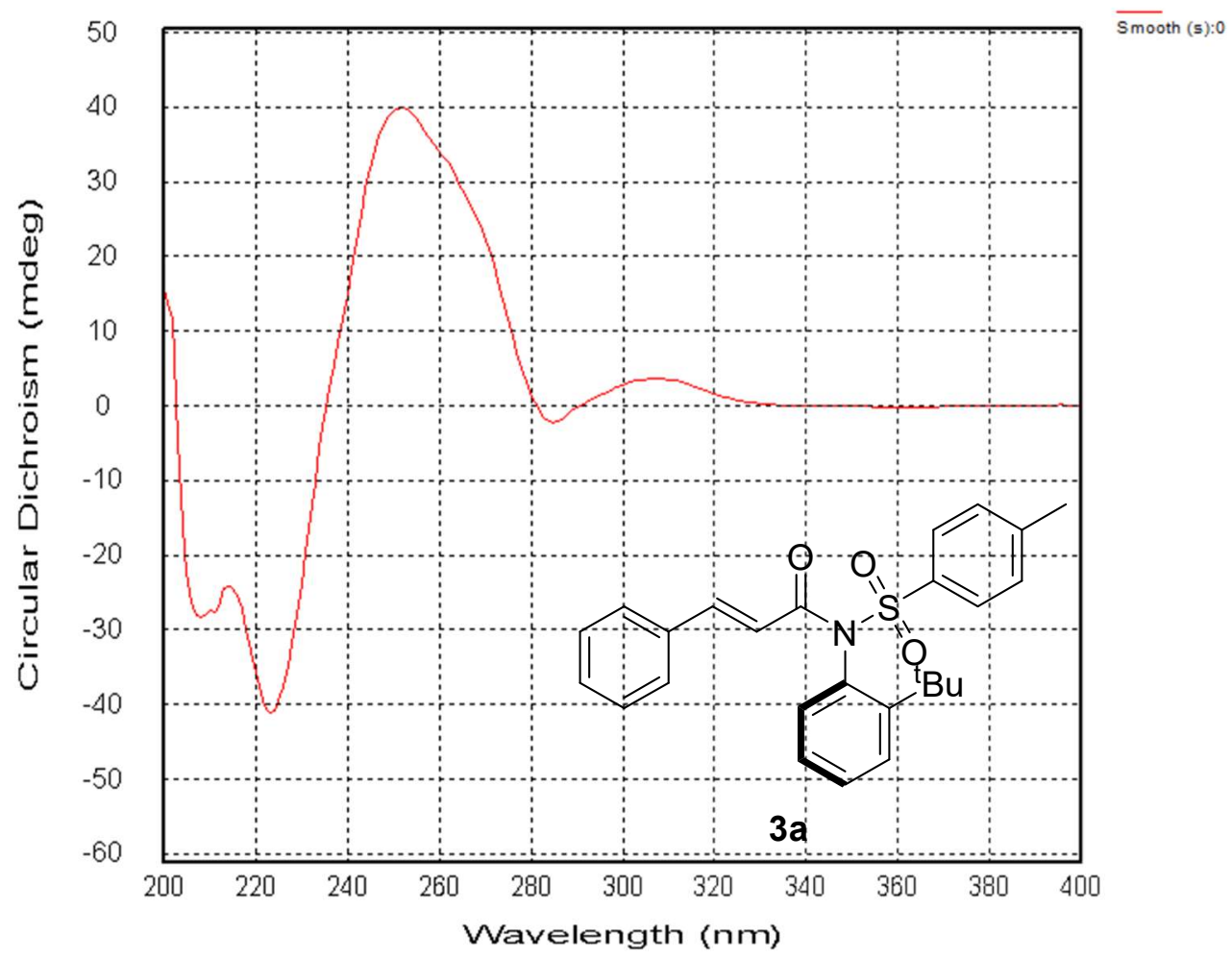




\section{References}

(1) Joannesse, C.; Johnston, C. P.; Concelln, C.; Simal, C.; Philp, D.; Smith, A. D. Isothiourea-Catalyzed Enantioselective Carboxy Group Transfer. Angew. Chem., Int. Ed. 2009, 48, 8914.

(2) (a) Fukata, Y.; Asano, K.; Matsubara, S. Facile Net Cycloaddition Approach to Optically Active 1,5-Benzothiazepines. J. Am. Chem. Soc. 2015, 137, 5320. (b) Liu, F. P.; Dong, Z.; Wang, J. C.; Dong, G. B. Palladium/Norbornene-Catalyzed Indenone Synthesis from Simple Aryl lodides: Concise Syntheses of Pauciflorol Fand Acredinone A. Angew.Chem., Int. Ed. 2019, $58,2144$.

(3) Nejrotti, S.; Ghinato, S.; Gini, E. C.; Scarpi, D.; Occhiato, E. G.; Maranzana, A.; Prandi, C. Gold(I)-Catalysed Hydroarylation of Lactam-Derived Enynes as an Entry to Tetrahydrobenzo[g]quinolines. Eur. J. Org. Chem. 2020, 646.

(4) Sheldrick, G. M. A short history of SHELX. Acta Cryst. 2008, A64, 112.

(5) Sheldrick, G. M. SHELXT-Integrated space-group and crystal-structure determination. Acta Cryst. 2015, A71, 3.

(6) Sheldrick, G. M. Crystal structure refinement with SHELXL. Acta Cryst. 2015, C71, 3.

(7) Dolomanov, O. V., Bourhis, L. J., Gildea, R. J., Howard, J. A. K., Puschmann, H. OLEX2: a complete structure solution, refinement and analysis program. J. Appl. Cryst. 2009, 42, 339. 